\title{
Optimization and Evaluation of Novel Antifungal Agents for the Treatment of Fungal Infection
}

Ji Won Choi ${ }^{1, \#, ~ K y u n g-T a e ~ L e e ~}{ }^{2, \#}$, Siwon Kim ${ }^{1,3}$, Ye Rim Lee ${ }^{1}$, Hyeon Ji Kim ${ }^{1}$, Kyung Jin $\mathrm{Seo}^{2}$, Myung Ha Lee ${ }^{2}$, Seul Ki Yeon ${ }^{1}$, Bo Ko Jang ${ }^{4}$, Sun Jun Park ${ }^{1,3}$, Hyeon Jeong Kim ${ }^{1,2}$, Jong-Hyun Park ${ }^{1,3}$, Dahee Kim ${ }^{4}$, Dong-Gi Lee ${ }^{4}$, Eunji Cheong ${ }^{2}$, Jong-Seung Lee,,*, YongSun Bahn ${ }^{2, *}$ and Ki Duk Park ${ }^{1,3^{*}}$

${ }^{1}$ Convergence Research Center for Diagnosis, Treatment \& Care System of Dementia, Korea Institute of Science \& Technology (KIST), Seoul 02792, Republic of Korea

${ }^{2}$ Department of Biotechnology, College of Life Science and Biotechnology, Yonsei University, Seoul 03722, Republic of Korea

${ }^{3}$ Division of Bio-Med Science \& Technology, KIST School, Korea University of Science and Technology, Seoul 02792, Republic of Korea

${ }^{4}$ Amtixbio Co., Ltd., Hanam-si, Gyeonggi-do 12925, Republic of Korea

\# These authors contributed equally to this work.

Address correspondence to: Ki Duk Park, Ph.D., ${ }^{1}$ Convergence Research Center for Diagnosis, Treatment \& Care System of Dementia, Korea Institute of Science \& Technology (KIST), Seoul, Republic of Korea; Email: kdpark@,kist.re.kr

Yong-Sun Bahn, Ph.D., ${ }^{2}$ Department of Biotechnology, College of Life Science and Biotechnology, Yonsei University, Seoul, Republic of Korea; Email: ysbahn@yonsei.ac.kr Jong-Seung Lee, ${ }^{4}$ AmtixBio Co., Ltd., Hanam-si, Gyeonggi-do, Republic of Korea; Email: jason_lee@amtixbio.com 


\section{Table of Contents}

1. Supplementary Results of Biology

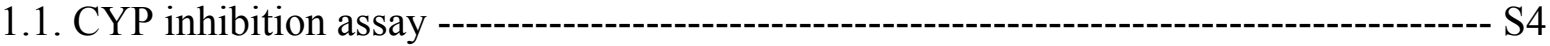

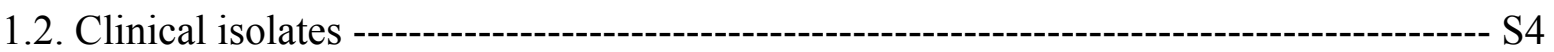

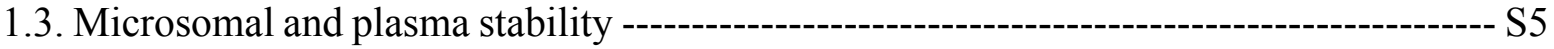

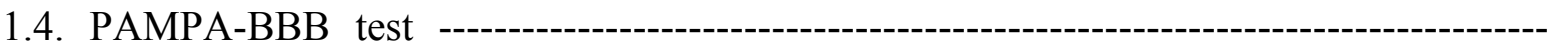
S5

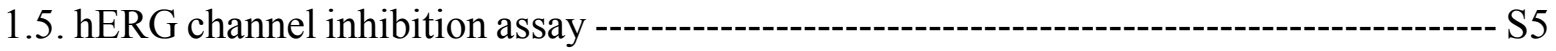

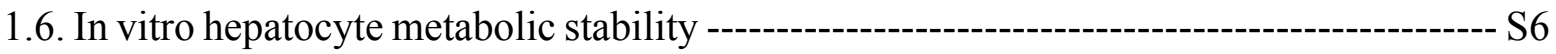

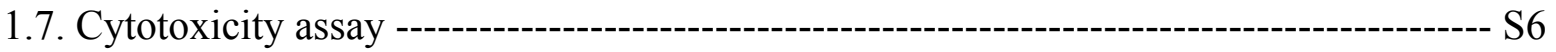

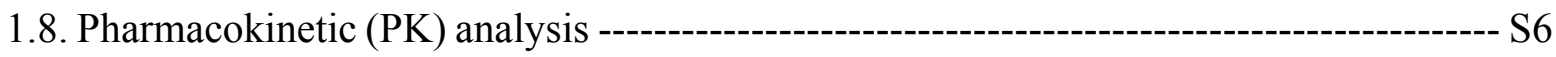

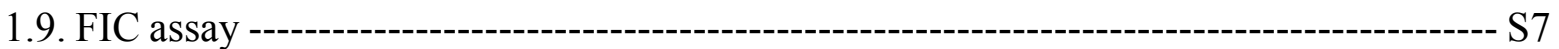

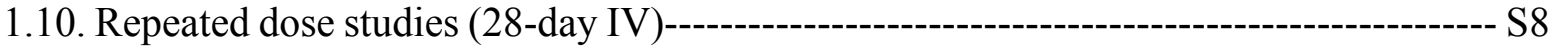

2. Supplementary Methods of Biology

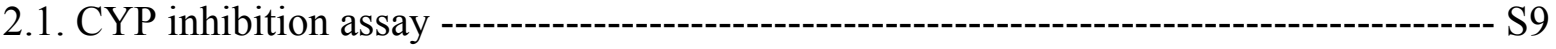

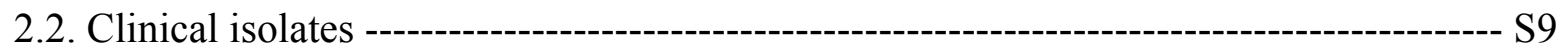

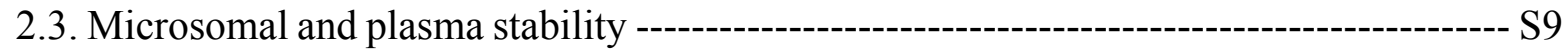

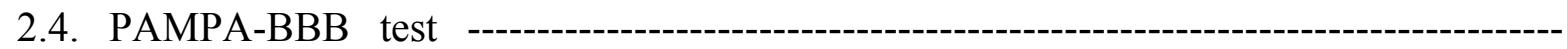
S 10

2.5. hERG channel inhibition assay -------------------------------------------------------------- S10

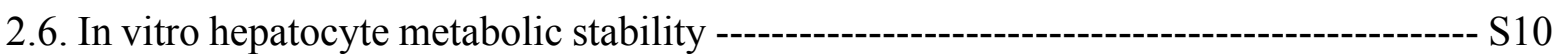

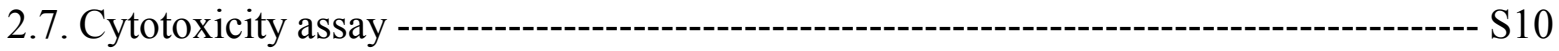

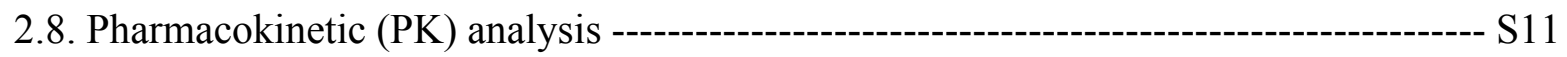

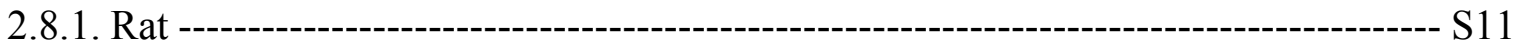

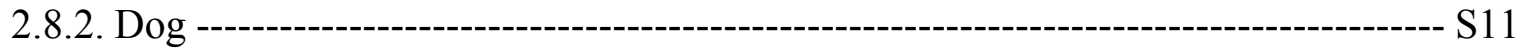

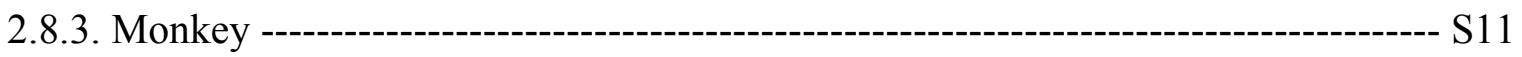

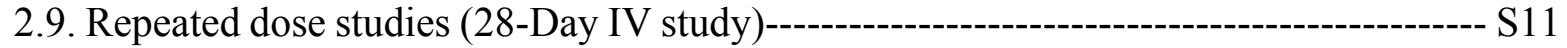


3. Supplementary Results of Chemistry

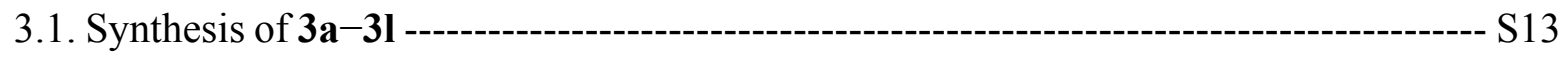

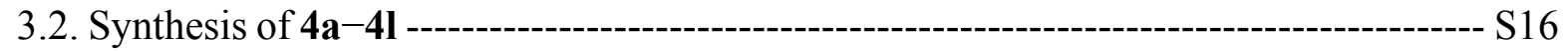

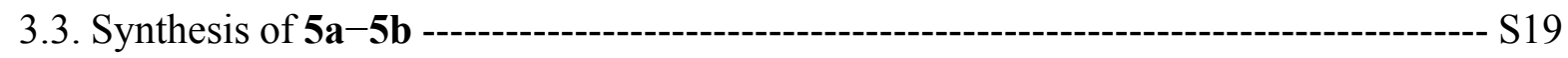

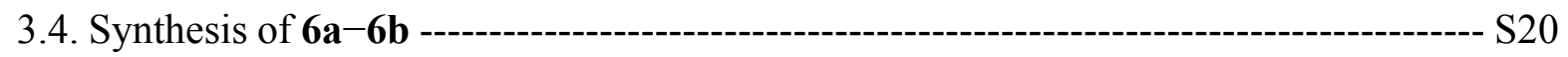

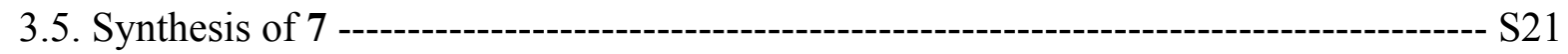

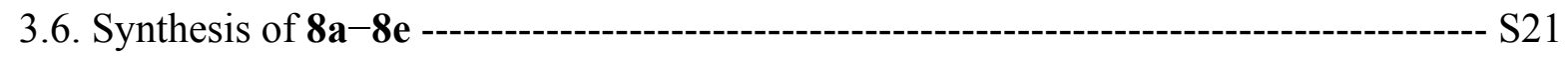

3.7. Synthesis of $\mathbf{9 a}-90$

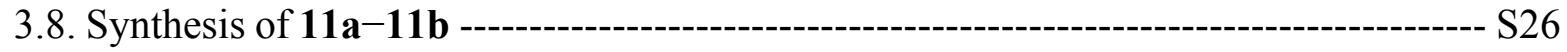

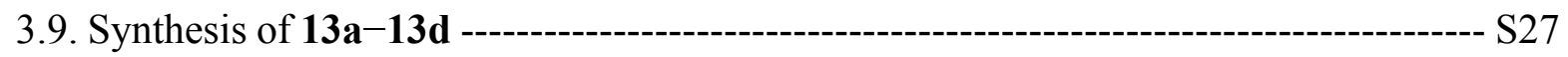

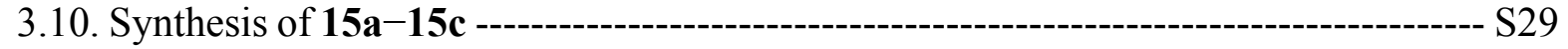

3.11. Synthesis of $\mathbf{1 6 a}-\mathbf{1 6 c}---$

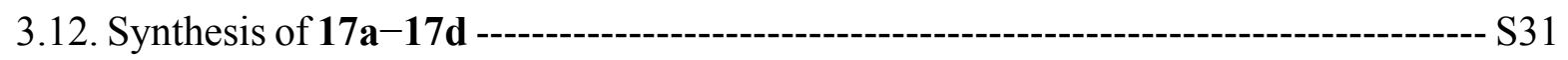

3.13. Synthesis of $\mathbf{1 9 a}-\mathbf{1 9 b}$

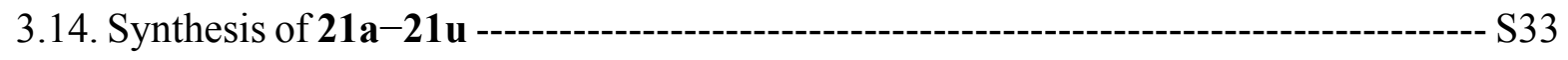

3.15. Synthesis of $\mathbf{2 3 a} \mathbf{2} \mathbf{2 3 b}----$

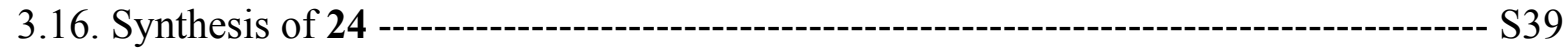

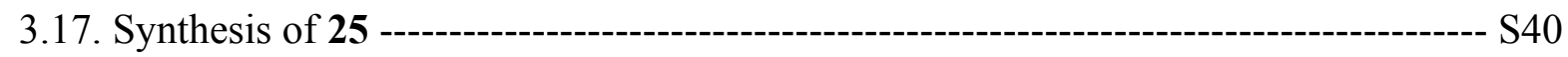

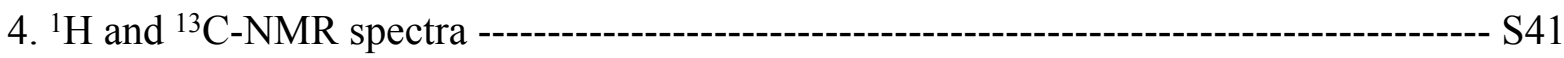

5. HRMS analysis ----------------------------------------------------------------------------------- S102

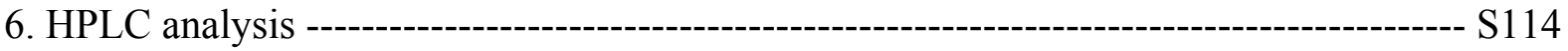




\section{Supplementary Results of Biology}

\subsection{CYP inhibition assay}

Table S1. CYP inhibition assay of the key compounds

\begin{tabular}{|c|c|c|c|c|c|c|c|c|c|c|c|}
\hline \multirow{2}{*}{ Compd. } & \multicolumn{5}{|c|}{ CYP enzyme activities $(\%)^{a}$} & \multirow{2}{*}{ Compd. } & \multicolumn{5}{|c|}{ CYP enzyme activities $(\%)^{a}$} \\
\hline & $1 \mathrm{~A} 2$ & $3 \mathrm{~A} 4$ & $2 \mathrm{C9}$ & $2 \mathrm{C} 19$ & 2D6 & & $1 \mathrm{~A} 2$ & $3 \mathrm{~A} 4$ & $2 \mathrm{C9}$ & $2 \mathrm{C} 19$ & 2 D6 \\
\hline $12 a$ & 70.4 & 67.0 & 37.5 & 85.3 & 91.7 & $22 j$ & 80.1 & 2.9 & 36.1 & 19.1 & 12.5 \\
\hline $12 b$ & 56.8 & 48.4 & 25.3 & 61.5 & 107.4 & $22 k$ & 28.9 & 12.7 & 63.2 & 40.6 & 25.6 \\
\hline $18 a(1)$ & 53.9 & 14.3 & 4.6 & 29.8 & 56.2 & 221 & 54.6 & 0.2 & 42.5 & 10.6 & 20.1 \\
\hline $18 b$ & 36.0 & 6.1 & 2.6 & 15.8 & 38.8 & $22 m$ & 85.2 & 48.9 & 50.3 & 43.0 & 11.8 \\
\hline $18 \mathrm{c}$ & 92.7 & 37.7 & 79.2 & 21.4 & 89.8 & $22 n$ & 90.9 & 40.0 & 69.4 & 38.7 & 5.5 \\
\hline $18 d$ & 81.2 & 17.5 & 49.8 & 10.5 & 77.0 & 220 & 75.3 & 24.1 & 51.7 & 43.5 & 16.5 \\
\hline $20 a$ & 98.9 & 2.2 & 62.6 & 15.1 & 15.7 & $22 p$ & 90.7 & 37.3 & 75.4 & 51.5 & 8.4 \\
\hline $20 b$ & 117.2 & 9.1 & 77.3 & 56.3 & 35.7 & $22 q$ & 94.8 & 35.4 & 73.9 & 28.0 & 2.9 \\
\hline $22 a$ & 77.3 & 11.4 & 57.3 & 41.5 & 16.0 & $22 r$ & 81.3 & 78.3 & 69.2 & 45.1 & 83.7 \\
\hline $22 b$ & 75.3 & 24.1 & 51.7 & 43.5 & 16.5 & $22 s$ & 102.4 & 22.6 & 49.6 & 20.3 & 1.2 \\
\hline $22 c$ & 23.7 & 13.3 & 78.7 & 29.8 & 40.5 & $22 t$ & 107.5 & 21.4 & 62.5 & 13.8 & 0.4 \\
\hline $22 d$ & 49.9 & 0.1 & 52.3 & 24.9 & 30.9 & $22 u$ & 68.6 & 9.2 & 36.6 & 10.4 & 4.6 \\
\hline $22 e$ & 79.6 & 9.9 & 63.6 & 46.6 & 31.0 & 26 & 82.4 & 24.2 & 25.0 & 49.8 & 79.9 \\
\hline $22 \mathrm{f}$ & 102.1 & 10.0 & 72.3 & 46.7 & 17.4 & 27 & 93.6 & 71.9 & 70.9 & 84.8 & 83.0 \\
\hline $22 \mathrm{~g}$ & 76.8 & 33.6 & 74.0 & 42.7 & 53.1 & $28 b$ & 79.6 & 14.8 & 88.3 & 59.5 & 13.0 \\
\hline $22 \mathrm{~h}$ & 81.1 & 43.7 & 77.3 & 86.0 & 88.7 & $30 \mathrm{~b}$ & 42.5 & 7.9 & 31.1 & 5.4 & 68.3 \\
\hline $22 i$ & 69.2 & 6.0 & 47.9 & 18.8 & 18.9 & 31 & 55.2 & 57.3 & 63.5 & 43.5 & 36.8 \\
\hline
\end{tabular}

${ }^{a}$ Cytochrome P450 (CYP) inhibition assay. The inhibitory capacity of each CYP subtypes by compounds was expressed as \% of control activity (without the compounds).

\subsection{Clinical Isolates}

Table S2. Antifungal effect of $22 \mathrm{~h}$ and two comparators on clinical isolates

\begin{tabular}{|c|c|c|c|c|c|}
\hline Species & $\begin{array}{c}\text { Antifungal } \\
\text { Drug }\end{array}$ & $\begin{array}{c}\text { MIC Range ( } \\
\mu \mathrm{g} / \mathrm{mL})\end{array}$ & $\begin{array}{c}\mathbf{M I C}_{50} *(\mu \\
\mathrm{g} / \mathrm{mL})\end{array}$ & $\begin{array}{c}\mathbf{M I C}_{\mathbf{9 0}_{0}} *(\mu \\
\mathrm{g} / \mathrm{mL})\end{array}$ & $\begin{array}{l}\text { No. of } \\
\text { Isolates }\end{array}$ \\
\hline \multirow{2}{*}{ Candia albicans } & $22 \mathrm{~h}$ & $1 \sim 8$ & 2 & 4 & \multirow{2}{*}{31} \\
\hline & Amphotericin B & $0.25 \sim 1$ & 0.25 & 0.5 & \\
\hline \multirow{3}{*}{ Candia glabrata } & $22 \mathrm{~h}$ & $2 \sim 8$ & 4 & 4 & \multirow{3}{*}{30} \\
\hline & Amphotericin B & $0.25 \sim 1$ & 0.5 & 1.0 & \\
\hline & Efinaconazole & $0.008 \sim>4$ & 0.016 & 0.031 & \\
\hline \multirow{3}{*}{$\begin{array}{l}\text { Cryptococcus } \\
\text { neoformans }\end{array}$} & $22 \mathrm{~h}$ & $2 \sim 16$ & 4 & 4 & \multirow{3}{*}{84} \\
\hline & Amphotericin B & $0.25 \sim>16$ & 0.5 & 1.0 & \\
\hline & Efinaconazole & $0.001 \sim>0.5$ & 0.004 & 0.008 & \\
\hline \multirow{3}{*}{$\begin{array}{l}\text { Aspergillus } \\
\text { fumigatus }\end{array}$} & $22 \mathrm{~h}$ & $4 \sim 16$ & 8 & 16 & \multirow{3}{*}{33} \\
\hline & Amphotericin B & $0.5 \sim 16$ & 4.0 & 8.0 & \\
\hline & Efinaconazole & $0.002 \sim 0.063$ & 0.016 & 0.063 & \\
\hline
\end{tabular}

Abbreviations: $\mathrm{MIC}=$ minimum inhibitory concentration; $\mathrm{MIC}_{50}=\mathrm{MIC}$ required to inhibit the growth of $50 \%$ of the isolates; $\mathrm{MIC}_{90}=$ MIC required to inhibit the growth of $90 \%$ of the isolates. 


\subsection{Microsomal and plasma stability}

Table S3. Microsomal and plasma stability of the key compounds

\begin{tabular}{|c|c|c|c|c|c|}
\hline \multirow{2}{*}{ Compd. } & \multicolumn{3}{|c|}{ Microsomal Stability $(\%)^{a}$} & \multicolumn{2}{|c|}{ Plasma Stability $(\%)^{b}$} \\
\hline & Human & Rat & Mouse & $\begin{array}{c}\text { Human } \\
(30,120 \mathrm{~min})\end{array}$ & $\begin{array}{c}\text { Rat } \\
(30,120 \mathrm{~min})\end{array}$ \\
\hline $22 \mathrm{~g}$ & 91.7 & 75.0 & 69.6 & $\mathrm{ND}^{c}$ & $\mathrm{ND}^{c}$ \\
\hline $22 \mathrm{~h}$ & 90.0 & 72.3 & 78.2 & $97.0,85.5$ & $96.2,79.1$ \\
\hline 221 & 97.1 & 74.2 & 85.7 & $\mathrm{ND}^{c}$ & $\mathrm{ND}^{c}$ \\
\hline $22 r$ & 95.0 & 44.0 & 67.0 & $97.1,87.3$ & $99.3,86.0$ \\
\hline $22 u$ & 87.6 & 44.8 & 57.2 & $\mathrm{ND}^{c}$ & $\mathrm{ND}^{c}$ \\
\hline
\end{tabular}

${ }^{a}$ In vitro profile of compounds in microsomal stability (Woburn, MA, USA); The incubation terminated prior to the addition of the NADPH regeneration system (time point 0 min) was used as standards defined as $100 \%$. Percentage of remaining compound determined by comparing the peak area after 30 min with microsomes. ${ }^{b} I n$ vitro profile of compounds plasma stability; \% remaining was determined after 30 min and 120 min incubation with human and rat plasma. ${ }^{c} \mathrm{ND}$ : not determined.

\subsection{PAMPA-BBB test}

Table S4. PAMPA-Blood-Brain Barrier (BBB) test of $22 \mathrm{~h}$

\begin{tabular}{cc}
\hline Compound & $\begin{array}{c}\text { BBB Permeability } \\
\left(\boldsymbol{P}_{\mathbf{e}}, \mathbf{c m} / \mathbf{s e c}\right)^{\boldsymbol{a}}\end{array}$ \\
\hline $\mathbf{2 2 h}$ & $58.944 \pm 1.244 \times 10^{-6}$ \\
Verapamil $^{b}$ & $87.156 \pm 1.511 \times 10^{-6}$ \\
Ranitidine $^{c}$ & $0.135 \pm 0.038 \times 10^{-6}$ \\
\hline
\end{tabular}

${ }^{a} P_{\mathrm{e}}$, Permeability was measured as the amount fo UV in the acceptor, donor and membrane using PAMPA explore program (version 3.8). ${ }^{b}$ Positive reference. ${ }^{c}$ Negative reference.

\section{5. hERG channel inhibition assay}

Table S5. hERG activity of the $22 \mathrm{~h}$

\begin{tabular}{cc}
\hline Compd. & hERG activity (\%) $^{a}$ \\
\cline { 2 - 2 } 22h & $3 \mu \mathrm{M}$ \\
\hline $\mathbf{0 . 0 1 \%}^{\text {DMSO }}{ }^{\boldsymbol{b}}$ & $39.502 \pm 5.223$ \\
\end{tabular}

${ }^{a}$ hERG activity: degree that does not inhibit. $\%=$ (current post compound treatment/current pre compound treatment) $\times 100 .{ }^{b} 0.01 \%$ DMSO: control. 


\subsection{In vitro hepatocyte metabolic stability}

Table S6. In vitro hepatocyte metabolic stability of $22 \mathrm{~h}$

\begin{tabular}{|c|c|c|c|c|c|}
\hline \multirow{2}{*}{ Compd. } & \multicolumn{5}{|c|}{ Metabolic Stability (\%) ${ }^{a}$ at $2 \mu \mathrm{M}$} \\
\hline & Sprague Dawly Rat & $\begin{array}{l}\text { New Zealand } \\
\text { White Rabbit }\end{array}$ & $\begin{array}{c}\text { Beagle } \\
\text { Dog }\end{array}$ & Gottingen Minipig & Human \\
\hline $22 \mathrm{~h}$ & 36.2 & 64.6 & 61.1 & 49.6 & 79.1 \\
\hline 7-Ethoxycoumarin ${ }^{b}$ & 65.1 & 0.1 & 3.7 & 82.4 & 63.8 \\
\hline Imipramineb $^{b}$ & 0.16 & 0.0 & 3.5 & 0.2 & 63.9 \\
\hline
\end{tabular}

${ }^{a} \%$ Remaining at $\mathrm{Time}_{60 \mathrm{~min}}=\left(\right.$ mean peak area ratio $\mathrm{T}_{60 \mathrm{~min}} /$ Mean peak area ratio $\left.\mathrm{T}_{0}\right) \times 100 .{ }^{b}$ references.

\subsection{Cytotoxicity assay}
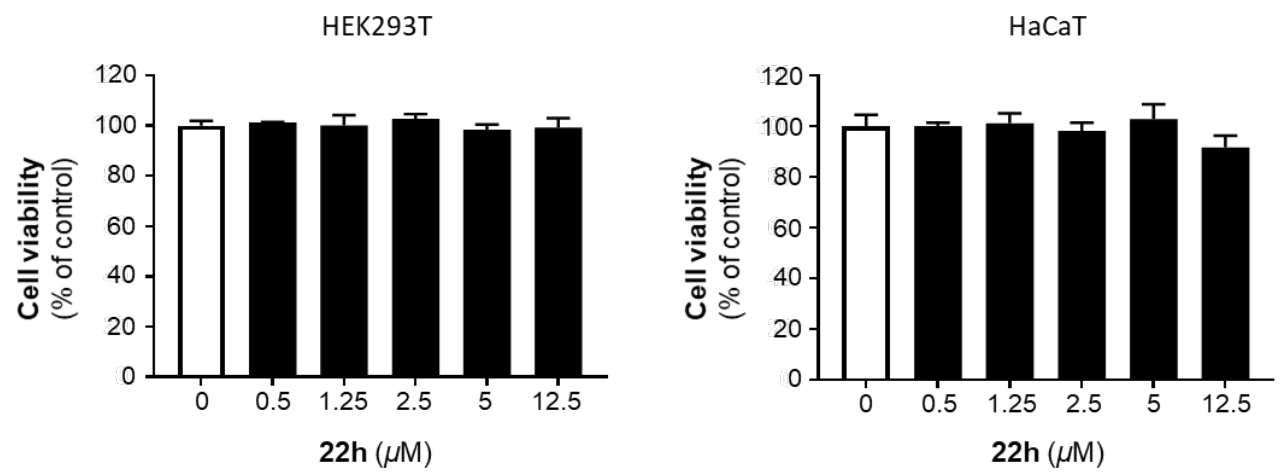

Figure S1. Cell viability after treatment of $\mathbf{2 2} \mathbf{h}$ for $24 \mathrm{~h}$ in human cell lines. The human cells were derived from human fetal cells (HEK293T) and human keratinocyte cells (HaCaT), respectively. Data are presented as mean $\pm \operatorname{SEM}(n=3)$. Data collection was repeated in three independent experiments compared with a vehicle-treated control (student's $t$-test).

\subsection{Pharmacokinetic (PK) analysis}

Table S7. Combined species PK parameters of $22 \mathrm{~h}$

\begin{tabular}{|c|c|c|c|c|c|c|c|c|c|c|}
\hline Species & Route & \begin{tabular}{|c|}
$\begin{array}{c}\text { Dose } \\
(\mathrm{mg} / \mathrm{kg})\end{array}$ \\
\end{tabular} & $\begin{array}{c}C_{\max } \\
(\mathrm{ng} / \mathrm{mL})\end{array}$ & $\begin{array}{c}A U C_{0-\mathrm{t}} \\
\text { (ng.hr/mL) }\end{array}$ & $\begin{array}{c}\boldsymbol{A U C} \boldsymbol{C}_{\infty} \\
(\mathrm{ng} . \mathbf{h r} / \mathbf{m L})\end{array}$ & $\begin{array}{c}t_{1 / 2} \\
(\mathbf{h r}) \\
\end{array}$ & $\begin{array}{c}M R T_{\infty} \\
(\mathrm{hr}) \\
\end{array}$ & $\begin{array}{c}\begin{array}{c}V_{\mathrm{d}} \\
(\mathrm{mL} / \mathrm{kg})\end{array} \\
\end{array}$ & \begin{tabular}{|c|c|}
$C L$ \\
$(\mathrm{ml} / \mathbf{h r} / \mathbf{k g})$
\end{tabular} & $\begin{array}{l}\boldsymbol{F} \\
\%\end{array}$ \\
\hline SD Rat & IV & 2 & 190.5 & 264.9 & 278.7 & 1.91 & 2.39 & 19846 & 7160 & \\
\hline SD Rat & $\mathrm{PO}$ & 10 & 97.34 & 526.7 & 677.1 & 2.84 & 5.78 & & & 39.8 \\
\hline Dog & IV & 1 & 296.3 & 2678 & 5158 & 24.27 & 34.62 & 7495 & 205.1 & \\
\hline Dog & IV & 10 & 4389 & 30527 & 50507 & 17.96 & 25.25 & 5148 & 202.0 & \\
\hline Dog & PO & 10 & 1366 & 23479 & 42092 & 19.59 & 29.24 & & & 76.9 \\
\hline Monkey & IV & 10 & 1083 & 7147 & 17355 & 31.29 & 42.9 & 27182 & 754.3 & \\
\hline
\end{tabular}


$A U C_{0-\mathrm{t}}=$ area under the concentration-time curve from time of dosing to the last quantifiable concentration; $A U C_{\infty}=$ area under the concentration curve from time zero to infinity; $C_{\max }=$ Maximum plasma concentration; $C L=$ clearance; $F=$ Bioavailability; IV = intravenous; MRT = mean residence time from time zero to infinity; $\mathrm{PO}=$ per os (oral); $t_{1 / 2}=$ half-life; $V_{\mathrm{d}}$ $=$ volume of distribution.

\subsection{FIC assay}

A Cryptococcus neoformans H99
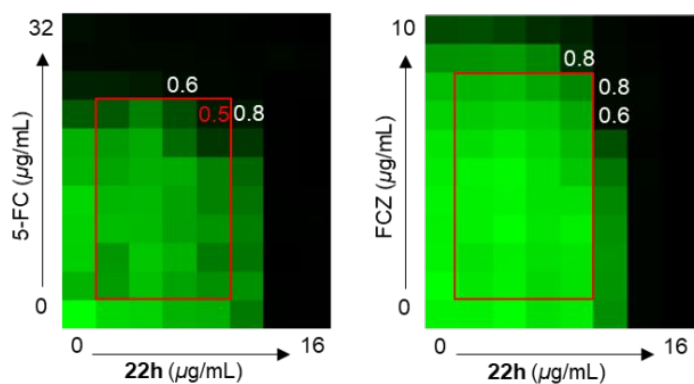

B

Candida albicans SC5314
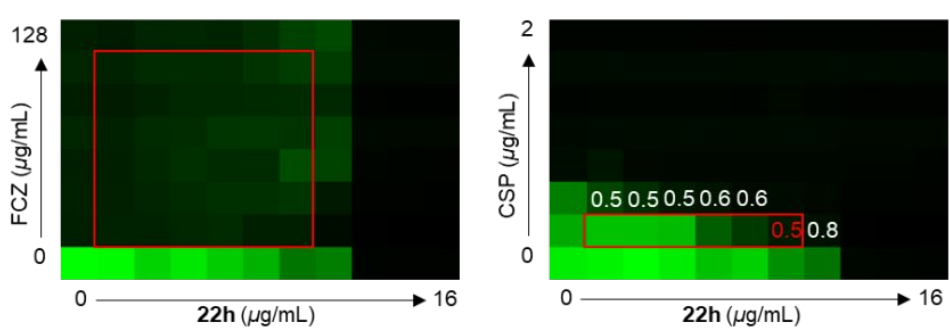

C

Candida auris B8441
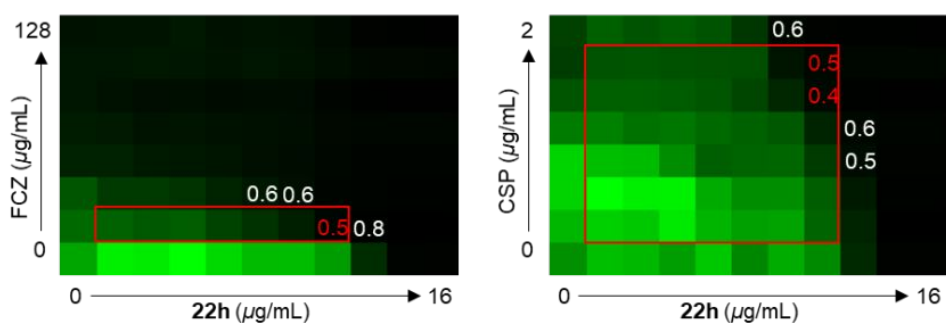

D Candida glabrata BG2
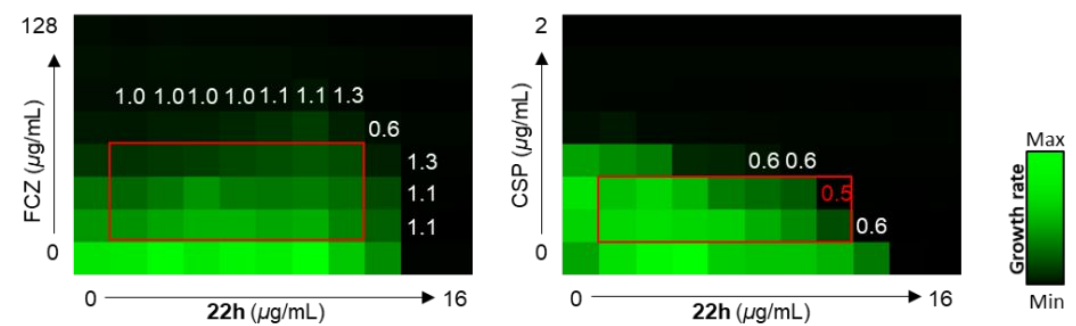

Figure S2. FIC assay of $22 \mathrm{~h}$ with clinically available antifungal drugs against invasive fungal pathogens. The checkerboard assays adhered to EUCAST guidelines. Flucytosine (5FC), fluconazole (FCZ), and caspofungin (CSP) were serially diluted at one side, and $\mathbf{2 2 h}$ was 
serially diluted on the other side of 96-well plates. The optical density at $600 \mathrm{~nm}$ (OD600) was determined using an iMark microplate absorbance reader and was used to determine cell growth. (A) C. neoformans (H99), (B) C. albicans (SC5314), (C) C. auris (B8441), and (D) C. glabrata (BG2) cells were incubated for $24 \mathrm{~h}$ in a $35^{\circ} \mathrm{C}$ incubator.

\subsection{Repeated dose studies (28-day IV)}

Table S8. Pharmacokinetic parameters in 28 day IV study

\begin{tabular}{|c|c|c|c|c|c|c|}
\hline Gender & \multicolumn{3}{|c|}{ Males } & \multicolumn{3}{|c|}{ Females } \\
\hline 22h Dose Level (mg/kg) & 3 & 10 & 15 & 3 & 10 & 15 \\
\hline$\underline{\text { Parameter Units }}$ & \multicolumn{6}{|c|}{ Day 1} \\
\hline$A U C_{(0-\mathrm{t})}(\mathrm{ng} \cdot \mathrm{h} / \mathrm{mL})$ & 360 & 1740 & 2480 & 365 & 1580 & 3180 \\
\hline$C_{0}(\mathrm{ng} / \mathrm{mL})$ & 139 & 1300 & 728 & 155 & 901 & 1430 \\
\hline$T_{\max }(\mathrm{h})$ & 0.5 & 0.5 & 1 & 0.5 & 0.5 & 0.5 \\
\hline$C L(\mathrm{~mL} / \mathrm{h} / \mathrm{kg})$ & 7750 & 5390 & 5120 & 7510 & 5940 & 4700 \\
\hline$V_{\mathrm{d}}(\mathrm{mL} / \mathrm{kg})$ & 23500 & 18400 & 21300 & 24700 & 1900 & 21700 \\
\hline$t_{1 / 2}$ & 2.10 & 2.36 & 2.88 & 2.28 & 2.22 & 3.2 \\
\hline$\underline{\text { Parameter Units }}$ & \multicolumn{6}{|c|}{ Day 28} \\
\hline$A U C_{(0-\mathrm{t})}(\mathrm{ng} \cdot \mathrm{h} / \mathrm{mL})$ & 448 & 3060 & 4110 & 441 & 2830 & 3550 \\
\hline$C_{0}(\mathrm{ng} / \mathrm{mL})$ & 169 & 778 & 987 & 208 & 713 & 1130 \\
\hline$T_{\max }(\mathrm{h})$ & 0.5 & 0.5 & 0.5 & 0.5 & 0.5 & 0.5 \\
\hline$C L(\mathrm{~mL} / \mathrm{h} / \mathrm{kg})$ & 5170 & 3260 & 3650 & 5300 & 3530 & 4230 \\
\hline$V_{\mathrm{d}}(\mathrm{mL} / \mathrm{kg})$ & 21100 & 13500 & 21500 & 21500 & 16900 & 17200 \\
\hline$t_{1 / 2}$ & 2.82 & 2.87 & 4.08 & 2.80 & 3.32 & 2.82 \\
\hline$R A U C$ & 1.25 & 1.76 & 1.66 & 1.21 & 1.80 & 1.12 \\
\hline
\end{tabular}

$A U C_{(0-\mathrm{t})}=$ area under the concentration-time curve from time of dosing to the last quantifiable concentration; $C L=$ clearance; $\mathrm{IV}=$ intravenous; $\mathrm{t}_{1 / 2}=$ half-life; $T_{\max }=$ Time of the maximum plasma concentration; $V_{\mathrm{d}}=$ volume of distribution. 


\section{Supplementary Methods of Biology}

\subsection{CYP inhibition assay}

The CYP inhibition assay was performed through luminescence analysis using the P450-Glo screening systems (Promega). The detailed method of this assay follows the protocol of Promega company (Technical Bulletin, P450-Glo Assays, Promega Corp., 2009). Briefly, each CYP enzyme and substrate mix were treated with or without synthesized compounds in potassium phosphate $\left(\mathrm{KPO}_{4}\right)$ buffer $(100 \mathrm{mM}, \mathrm{pH}$ 7.4). The reaction of mixure was initiated by adding the NADPH regenerating system containing glucose-6-phosphate, NADP ${ }^{+}$, magnesium chloride $\left(\mathrm{MgCl}_{2}\right)$ and glucose-6-phosphate dehydrogenase. The reaction was incubated at $37{ }^{\circ} \mathrm{C}$ for $10-30 \mathrm{~min}$ (different incubation times according to the isotype), and then treated with the detection reagent (reconstituted luciferin) to stop the reaction. After incubation for $20 \mathrm{~min}$ to stabilize the luminescence signal, the signal was detected using a microplate reader (SpectraMax ${ }^{\circledR} i 3$, Molecular Devices), and the data was reported as relative light unit (RLU).

\subsection{Clinical isolates}

All information for the clinical fungal isolates used in MIC analysis was listed in the Supplementary Data as an Excel file. We obtained 31 C. albicans strains, 30 C. glabrata strains, and 33 A. fumigatus strains from the Korean Collection of Medical Fungi in Konyang University. We also tested 84 C. neoformans strains that were previously published (6 from Bahn et al; 78 from Choi et al). ${ }^{1,2}$ The cell number was adjusted in RPMI 1640 broth (pH 7.0 with MOPS buffer) as to $1.5 \times 10^{3}$ cells $/ \mathrm{mL}$ for Cryptococcus and Candida species, or to $1.5 \times 10^{4}$ conidia $/ \mathrm{mL}$ for Aspergillus, and incubated at $35^{\circ} \mathrm{C}$ for $48 \mathrm{~h}$ (Candida species) or $72 \mathrm{~h}$ (Cryptococcus and Aspergillus). Endpoint readings were performed visually based on comparison of the fungal growth in treatment and control group. For $\mathbf{2 2} \mathbf{h}$ and AmB, the MIC was defined as the lowest concentration showing $100 \%$ growth inhibition. For ECZ, 50\% growth inhibition relative to the drug-free growth control was defined as $\mathrm{MIC}\left(\mathrm{IC}_{50}\right)$ due to the characteristic of this azole class antifungal agent. $\mathrm{MIC}_{50}$ and $\mathrm{MIC}_{90}$ values were defined as the MICs required to inhibit the growth of $50 \%$ and $90 \%$ of the isolates, respectively

\subsection{Microsomal and plasma stability}

To evaluate the microsomal stability for key compounds, the microsomes $(0.5 \mathrm{mg} / \mathrm{mL}$, Corning ${ }^{\circledR}$ UltraPool ${ }^{\mathrm{TM}}$ HLM150) were reacted by adding the NADPH regenerating system containing glucose-6-phosphate $(10 \mathrm{mM})$, glucose-6-phosphate dehydrogenase $(0.2 \mathrm{U} / \mathrm{mL})$ and $\mathrm{MgCl}_{2}(9.2 \mathrm{mM})$. The reaction mixure was incubated for $5 \mathrm{~min}$ at $37^{\circ} \mathrm{C}$, and then was treated with NADPH (1.2 $\mathrm{mM})$. The samples were collected after 0 and 30 min reaction, and then extracted with acetonitrile. The supernatants were obtained using centrifugation at $10,000 \times g$ for $10 \mathrm{~min}$ and analyzed by LC/MS/MS to determine the remaining compounds. The human and rat plasma (Sigma-Aldrich) was treated with $\mathbf{2 2 h}$ to evaluate the plasma stability. The samples were collected after $30 \mathrm{~min}$ reaction, and then extracted with acetonitrile. The 
supernatants were obtained using centrifugation at $10,000 \times \mathrm{g}$ for $5 \mathrm{~min}$ and analyzed by $\mathrm{LC} / \mathrm{MS} / \mathrm{MS}$ to determine the remaining $\mathbf{2 2} \mathbf{h}$.

\subsection{PAMPA-BBB (Parallel Artificial Membrane Permeability Assay-Blood-Brain Barrier) test}

PAMPA-BBB was measured using Pion Parallel Artificial Memebrane Permeability Assay Explorer (version 3.8) according to the manufacturer's protocols. 22h was treated with donor buffer in deep-well plates and an inverted acceptor plate. Membranes were wetted with lipid solution and treated with acceptor sink buffer in each well. Acceptor and donor plates were combined and incubated at $25^{\circ} \mathrm{C}$ for $4 \mathrm{~h}$. After, acceptor and donor plates were separated, transferred to ultraviolet plate in each well, and the ultraviolet value was measured using the Parallel Artifical Memebrane Permeability Assay explore program. $P_{e}$ classifications: high, $P_{e}$ $>1.5 \times 10^{-6} \mathrm{~cm} / \mathrm{s}$; low, $P_{e}<1.5 \times 10^{-6} \mathrm{~cm} / \mathrm{s}$.

\section{5. hERG channel inhibition assay}

The hERG channel inhibition assay was performed according to the manufacturer's protocol using hERG fluorescence polarization assay (\#PV5365, Invitrogen). Briefly, hERG membrane was treated with $\mathbf{2 2 h}$, fluorescence tracer red dye and fluorescence polarization buffer, and then incubated for $4 \mathrm{~h}$ at $25^{\circ} \mathrm{C}$. The fluorescent signal (Ex. $530 \mathrm{~nm}$, Em. $\left.590 \mathrm{~nm}\right)$ was detected using a multi-mode microplate reader (Synergy Neo, Biotek).

\subsection{In vitro hepatocyte metabolic stability}

The purpose of non-GLP study was to determine the metabolic stability of $\mathbf{2 2} \mathbf{h}$ in the presence of Sprague-Dawley rat, New Zealand White rabbit, beagle dog, Gottingen minipig, and human hepatocytes. The frozen hepatocytes were thawed with warm InVitroGRO HT medium (cas No. Z99019) and resuspended in InVitroGRO KHB buffer. The candidate 22h and controls (7ethoxycoumarin and imipramine) were treated at the concentration of $2 \mu \mathrm{M}$ in each matrix $\left(5 \times 10^{5}\right.$ cells $\left./ \mathrm{mL}\right)$ in 96-well plates. And afterwards, samples were incubated for $2 \mathrm{~h}$ at $37{ }^{\circ} \mathrm{C}$. The samples were analyzed by LC-MS/MS equipment at the time points $(0,15,30,45,60$ and $120 \mathrm{~min}$ ), repectively. Data were expressed as the peak area ratio (analyte/internal standard).

\subsection{Cytotoxicity assay}

Cell viability test was performed on human cell lines (HEK293T and HaCaT) using the EzCytox cell viability assay kit (DoGenBio, Seoul, South Korea) according to previously reported. ${ }^{3,4} \mathrm{HaCaT}$ cells were obtained as the immortalized keratinocytes from normal skin. The cells $\left(1.5 \times 10^{4}\right.$ cells/well $)$ were seeded in 96-well plates and incubated for $24 \mathrm{~h}$ at $37{ }^{\circ} \mathrm{C}$ containing $5 \% \mathrm{CO}_{2}$. Cells were treated with various concentrations $(0.5-12.5 \mu \mathrm{M}$, final concentration) of $\mathbf{2 2 h}$ for $24 \mathrm{~h}$ incubation. After incubation, Ez-Cytox reagent was added and 
incubated for $1 \mathrm{~h}$ at $37{ }^{\circ} \mathrm{C}$. The viability was measured by absorbance at $450 \mathrm{~nm}$ using a microplate reader (SpectraMax ${ }^{\circledR} i 3$, Molecular Device).

\subsection{Pharmacokinetic (PK) analysis}

\subsubsection{Rat}

22h was formulated in water for injection for administration via the oral and IV route. SD rats ( $\mathrm{n}=4$ males/group) were administered either $10 \mathrm{mg} / \mathrm{kg}$ orally or $2 \mathrm{mg} / \mathrm{kg}$ via IV. Blood samples were collected via jugular vein at pre-dose and post-dose at 5, 15, and $30 \mathrm{~min}, 1,2,4,6,8$, and $24 \mathrm{~h}$. Plasma was prepared and stored frozen at $-80{ }^{\circ} \mathrm{C}$ until bioanalysis. $\mathbf{2 2 h}$ was measured in plasma using liquid chromatography-tandem mass spectrometry (LC-MS)/MS assay with a lower limit of quantification (LLOQ) of $1 \mathrm{ng} / \mathrm{mL}$.

\subsubsection{Dog}

Beagle dogs (3 males/dose) were orally administered a $10 \mathrm{mg} / \mathrm{kg}$ dose of $\mathbf{2 2 h}$ formulated in sterile water for injection. Blood samples were collected via jugular vein at at pre-dose and after dosing at 5, 15, and $30 \mathrm{~min}, 1,2,4,6,8$, and $24 \mathrm{~h}$. Plasma was collected and frozen at $80{ }^{\circ} \mathrm{C}$ until analysis. $\mathbf{2 2} \mathbf{h}$ was measured in plasma using a LC-MS/MS assay with a limit of LLOQ of $5 \mathrm{ng} / \mathrm{mL}$.

Beagle dogs ( 3 males/dose) were administered 1 and $10 \mathrm{mg} / \mathrm{kg} \mathrm{22h}$ formulated in water for injection and was administered as a single IV infusion over $10 \mathrm{~min}$. Blood was collected predose and at 5, 15 and $30 \mathrm{~min}, 1,2,4,6,8$ and $24 \mathrm{~h}$ after dosing. Plasma was collected at stored at $-80{ }^{\circ} \mathrm{C}$ prior to analysis. $22 \mathrm{~h}$ was measured using an LC-MS/MS assay with a limit of LLOQ of $10 \mathrm{ng} / \mathrm{mL}$.

\subsubsection{Monkey}

Cynomolgus monkeys ( $\mathrm{n}=3$ males) were administered $10 \mathrm{mg} / \mathrm{kg} \mathbf{2 2} \mathbf{h}$ formulated in water for injection, as a single IV infusion over $10 \mathrm{~min}$. Blood, (approximately $\sim 1 \mathrm{~mL} /$ sample) was collected via jugular vein at pre-dose and after dosing at 5, 15, and $30 \mathrm{~min}, 1,2,4,8,10$, and $24 \mathrm{~h}$. Plasma was separated and stored frozen prior to analysis. $22 \mathbf{h}$ was measured in plasma using an LC-MS/MS assay with a limit of LLOQ of $5 \mathrm{ng} / \mathrm{mL}$.

\subsection{Repeated dose studies (28-Day IV study)}

As a part of the 28-day repeated dose general toxicology study, SD rats ( $n=6 /$ sex/dose) were administered 3,10 , or $15 \mathrm{mg} / \mathrm{kg}$ by slow IV bolus injection in a surgically implanted femoral vein catheter. A concurrent control group ( $n=3 / \operatorname{sex})$ received vehicle (sterile water for injection) following the same dosing regimen. The dose volume was $5 \mathrm{~mL} / \mathrm{kg}$ for all groups.

Toxicokinetic analysis occurred on Day 1 and 28 (final day). Blood samples (approx. $0.3 \mathrm{~mL}$ ) were collected from the jugular vein under isoflurane anesthesia. 22h treated animals $(\mathrm{n}=3$ at each timepoint) were collected at pre-dose and, after dosing at 0.5, 1, 2, 8, and $24 \mathrm{~h}$. Control animals were collected at pre-dose and at $2 \mathrm{~h}$. Plasma samples were stored at $-70{ }^{\circ} \mathrm{C}$ until analyzed. Plasma concentrations of $\mathbf{2 2} \mathbf{h}$ were measured using a validated LC-MS/MS method. 


\section{References}

1. Bahn, Y. S.; Kojima, K.; Cox, G. M.; Heitman, J. Specialization of the HOG Pathway and Its Impact on Differentiation and Virulence of Cryptococcus neoformans. Mol. Biol. Cell. 2005, $16(5), 2285-2300$.

2. Choi, Y. H.; Ngamskulrungroj, P.; Varma, A.; Sionov, E.; Hwang, S. M.; Carriconde, F.; Meyer, W.; Litvintseva, A. P.; Lee, W. G.; Shin, J. H.; Kim, E. C.; Lee, K. W.; Choi, T. Y.; Lee, Y. S.; Kwon-Chung, K. J. Prevalence of the VNIc Genotype of Cryptococcus neoformans in non-HIV-associated Cryptococcosis in the Republic of Korea. FEMS. Yeast. Res. 2010, 10(6), 769-778.

3. Choi, J. W.; Kim, S.; Park, J.-H.; Kim, H. J.; Shin, S. J.; Kim, J. W.; Woo, S. Y.; Lee, C.; Han, S. M.; Lee, J.; Pae, A. N.; Han, G.; Park, K. D.*; Optimization of Vinyl Sulfone Derivatives as Potent Nuclear Factor Erythroid 2-related Factor 2 (Nrf2) Activators for Parkinson's Disease Therapy. J. Med. Chem. 2018, 62, 811-830

4. Choi, J. W.; Shin, S. J.; Kim, H. J.; Park, J.-H.; Kim, H. J.; Lee, E. H.; Pae, A. N.; Bahn, Y. S.; Park, K. D.*; Antioxidant, Anti-inflammatory, and Neuroprotective Effects of Novel Vinyl Sulfonate Compounds as Nrf2 Activator. ACS Med. Chem. Lett. 2019, 10, 1061-1067 


\section{Supplementary Results of Chemistry}

Scheme S1. Synthetic route for 4 .

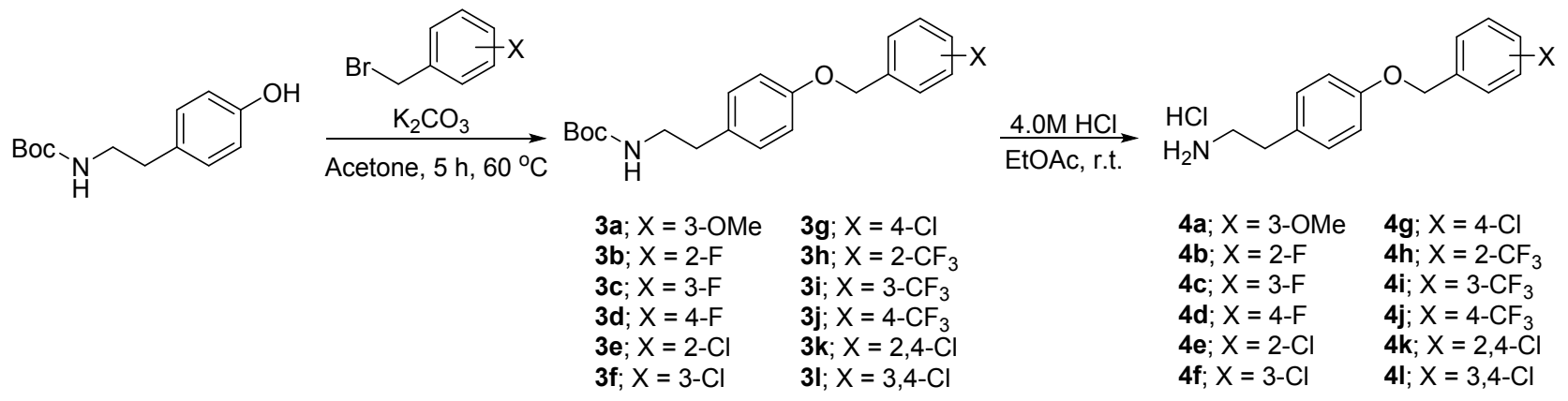

\subsection{Synthesis of 3}

tert-Butyl (4-((3-methoxybenzyl)oxy)phenethyl)carbamate (3a)

Using Method A, $N$-(tert-Butoxycarbonyl)tyramine (1.00 g, $4.21 \mathrm{mmol})$, 3-methoxybenzyl bromide $(0.71 \mathrm{~mL}, 5.06 \mathrm{mmol})$ and $\mathrm{K}_{2} \mathrm{CO}_{3}(2.33 \mathrm{~g}, 16.9 \mathrm{mmol})$ in acetone (42 $\left.\mathrm{mL}\right)$ gave 3a as a white solid (1.31 g, 87\%); $R_{f}=0.40$ (EtOAc $1: n$-hexane 3$) ;{ }^{1} \mathrm{H}$ NMR $\left(400 \mathrm{MHz}, \mathrm{CDCl}_{3}\right) \delta$ 7.32-7.25 (m, 1 ArH), 7.10 (d, $J=8.4$ Hz, 2 ArH), 7.00 (d, J=7.3 Hz, 2 ArH), 6.93-6.84 (m, $3 \mathrm{ArH}), 5.01$ (s, OCH $\mathbf{O H}_{2}, 4.56$ (br s, NH), $3.81\left(\mathrm{~s}, \mathrm{OCH}_{3}\right), 3.34-3.32\left(\mathrm{~m}, \mathrm{NHCH}_{2} \mathrm{CH}_{2}\right), 2.72$ (t, $\left.J=6.9 \mathrm{~Hz}, \mathrm{NHCH}_{2} \mathrm{CH}_{2}\right), 1.43\left(\mathrm{~s}, \mathrm{C}\left(\mathrm{CH}_{3}\right)_{3}\right) ;{ }^{13} \mathrm{C} \mathrm{NMR}\left(75 \mathrm{MHz}, \mathrm{CDCl}_{3}\right) \delta 159.9,157.5(\mathrm{ArC})$, $155.9(\mathbf{C}(\mathrm{O})), 138.8,131.4,129.8,129.7,119.6,115.0,113.5,112.9(\mathrm{ArC}), 79.2\left(\mathbf{C}\left(\mathrm{CH}_{3}\right)_{3}\right)$, $70.0\left(\mathrm{OCH}_{2}\right), 55.3\left(\mathrm{OCH}_{3}\right), 42.0\left(\mathrm{NHCH}_{2} \mathrm{CH}_{2}\right), 35.4\left(\mathrm{NHCH}_{2} \mathbf{C H}_{2}\right), 28.4\left(\mathrm{C}_{\left.\left(\mathrm{CH}_{3}\right)_{3}\right)}\right.$

\section{tert-Butyl (4-((2-fluorobenzyl)oxy)phenethyl)carbamate (3b)}

Using Method A, N-(tert-butoxycarbonyl)tyramine (1.00 g, $4.21 \mathrm{mmol})$, 2-fluorobenzyl bromide $(0.53 \mathrm{~mL}, 4.42 \mathrm{mmol})$ and $\mathrm{K}_{2} \mathrm{CO}_{3}(2.33 \mathrm{~g}, 16.9 \mathrm{mmol})$ in acetone $(42 \mathrm{~mL})$ gave $3 \mathbf{b}$ as a white solid $(0.97 \mathrm{~g}, 66 \%) ; R_{f}=0.48($ EtOAc $1: n$-hexane 3$) ;{ }^{1} \mathrm{H}$ NMR $\left(400 \mathrm{MHz}, \mathrm{CDCl}_{3}\right) \delta$ 7.50 (t, $J=7.2 \mathrm{~Hz}, 1$ ArH), 7.33-7.28 (m, 1 ArH), 7.17-7.06 (m, 4 ArH), 6.94-6.92 (m, 2 ArH), 5.11 (s, OCH $\left.\mathbf{O H}_{2}\right), 4.55$ (br s, NH), 3.35-3.33 (m, $\left.\mathrm{NHCH}_{2} \mathrm{CH}_{2}\right), 2.73$ (t, $J=6.8 \mathrm{~Hz}, \mathrm{NHCH}_{2} \mathrm{CH}_{2}$ ), $1.43\left(\mathrm{~s}, \mathrm{C}\left(\mathrm{CH}_{3}\right)_{3}\right) ;{ }^{13} \mathrm{C} \mathrm{NMR}\left(75 \mathrm{MHz}, \mathrm{CDCl}_{3}\right) \delta 160.5\left(\mathrm{~d}, J_{\mathrm{C}-\mathrm{F}}=245.4 \mathrm{~Hz}\right), 158.8(\mathrm{ArC}), 155.9$ $(\mathbf{C}(\mathrm{O})), 131.6,129.8,129.7\left(\mathrm{~d}, J_{\mathrm{C}-\mathrm{F}}=1.4 \mathrm{~Hz}\right), 129.6\left(\mathrm{~d}, J_{\mathrm{C}-\mathrm{F}}=5.8 \mathrm{~Hz}\right), 124.3\left(\mathrm{~d}, J_{\mathrm{C}-\mathrm{F}}=14.1\right.$ $\mathrm{Hz}), 124.2\left(\mathrm{~d}, J_{\mathrm{C}-\mathrm{F}}=3.6 \mathrm{~Hz}\right), 115.3\left(\mathrm{~d}, J_{\mathrm{C}-\mathrm{F}}=21.1 \mathrm{~Hz}\right), 115.0,124.2,115.5,115.2,115.0(\mathrm{ArC})$, $79.2\left(\mathbf{C}\left(\mathrm{CH}_{3}\right)_{3}\right), 63.8\left(\mathrm{OCH}_{2}\right), 42.0\left(\mathrm{NHCH}_{2} \mathrm{CH}_{2}\right), 35.4\left(\mathrm{NHCH}_{2} \mathrm{CH}_{2}\right), 28.4\left(\mathrm{C}_{\left.\left(\mathrm{CH}_{3}\right)_{3}\right)}\right)$.

\section{tert-Butyl (4-((3-fluorobenzyl)oxy)phenethyl)carbamate (3c)}

Using Method A, N-(tert-butoxycarbonyl)tyramine (1.00 g, $4.21 \mathrm{mmol}), 3$-fluorobenzyl bromide $(0.57 \mathrm{~mL}, 4.64 \mathrm{mmol})$ and $\mathrm{K}_{2} \mathrm{CO}_{3}(2.33 \mathrm{~g}, 16.9 \mathrm{mmol})$ in acetone (42 $\left.\mathrm{mL}\right)$ gave $3 \mathbf{c}$ as a white solid (1.06 g, 73\%); $R_{f}=0.47$ (EtOAc $1: n$-hexane 3$) ;{ }^{1} \mathrm{H}$ NMR $\left(300 \mathrm{MHz}, \mathrm{CDCl}_{3}\right) \delta$ 7.38-7.30 (m, 1 ArH), 7.20-7.10 (m, 4 ArH), 7.04-7.00 (m, 1 ArH), 6.92-6.89 (m, 2 ArH), 5.04 
(s, $\left.\mathrm{OCH}_{2}\right), 4.53$ (br s, NH), 3.35-3.33 (m, $\left.\mathrm{NHCH}_{2} \mathrm{CH}_{2}\right), 2.73\left(\mathrm{t}, J=6.9 \mathrm{~Hz}, \mathrm{NHCH}_{2} \mathrm{CH}_{2}\right), 1.43$ $\left(\mathrm{s}, \mathrm{C}\left(\mathrm{CH}_{3}\right)_{3}\right) ;{ }^{13} \mathrm{C} \mathrm{NMR}\left(75 \mathrm{MHz}, \mathrm{CDCl}_{3}\right) \delta 163.0\left(\mathrm{~d}, J_{\mathrm{C}-\mathrm{F}}=244.7 \mathrm{~Hz}\right), 157.2(\mathrm{ArC}), 155.9$ $(\mathbf{C}(\mathrm{O})), 139.8\left(\mathrm{~d}, J_{\mathrm{C}-\mathrm{F}}=7.3 \mathrm{~Hz}\right), 131.6,130.1\left(\mathrm{~d}, J_{\mathrm{C}-\mathrm{F}}=8.2 \mathrm{~Hz}\right), 129.8,122.7\left(\mathrm{~d}, J_{\mathrm{C}-\mathrm{F}}=2.8 \mathrm{~Hz}\right)$,

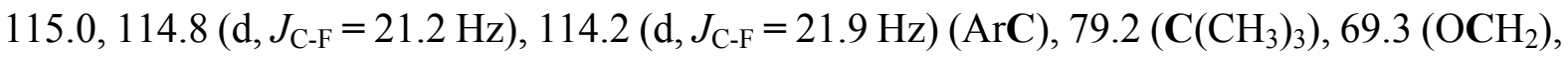
$42.0\left(\mathrm{NHCH}_{2} \mathrm{CH}_{2}\right), 35.4\left(\mathrm{NHCH}_{2} \mathrm{CH}_{2}\right), 28.4\left(\mathrm{C}\left(\mathrm{CH}_{3}\right)_{3}\right)$.

\section{tert-Butyl (4-((4-fluorobenzyl)oxy)phenethyl)carbamate (3d)}

Using Method A, N-(tert-butoxycarbonyl)tyramine (1.00 g, $4.21 \mathrm{mmol})$, 4-fluorobenzyl bromide $(0.58 \mathrm{~mL}, 4.64 \mathrm{mmol})$ and $\mathrm{K}_{2} \mathrm{CO}_{3}(2.33 \mathrm{~g}, 16.9 \mathrm{mmol})$ in acetone (42 mL) gave $3 \mathbf{d}$ as a white solid (1.36 g, 93\%); $R_{f}=0.41$ (EtOAc $1: n$-hexane 3$) ;{ }^{1} \mathrm{H}$ NMR $\left(300 \mathrm{MHz}, \mathrm{CDCl}_{3}\right) \delta$ 7.42-7.36 (m, 2 ArH), 7.12-7.03 (m, 4 ArH), 6.92-6.88 (m, 2 ArH), 5.00 (s, OCH $), 4.54$ (br $\mathrm{s}, \mathrm{NH}), 3.35-3.33\left(\mathrm{~m}, \mathrm{NHCH}_{2} \mathrm{CH}_{2}\right), 2.73\left(\mathrm{t}, J=7.0 \mathrm{~Hz}, \mathrm{NHCH}_{2} \mathrm{CH}_{2}\right), 1.43\left(\mathrm{~s}, \mathrm{C}\left(\mathrm{CH}_{3}\right)_{3}\right) ;{ }^{13} \mathrm{C}$ NMR (75 MHz, $\left.\mathrm{CDCl}_{3}\right) \delta 162.5\left(\mathrm{~d}, J_{\mathrm{C}-\mathrm{F}}=244.5 \mathrm{~Hz}\right), 157.3(\mathrm{ArC}), 155.9(\mathbf{C}(\mathrm{O})), 132.9,131.5$,

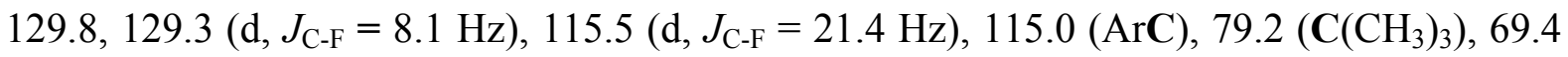
$\left(\mathrm{OCH}_{2}\right), 42.0\left(\mathrm{NHCH}_{2} \mathrm{CH}_{2}\right), 35.4\left(\mathrm{NHCH}_{2} \mathbf{C H}_{2}\right), 28.4\left(\mathrm{C}\left(\mathrm{CH}_{3}\right)_{3}\right)$.

\section{tert-Butyl (4-((2-chlorobenzyl)oxy)phenethyl)carbamate (3e)}

Using Method A, N-(tert-butoxycarbonyl)tyramine (1.00 g, $4.21 \mathrm{mmol})$, 2-chlorobenzyl bromide $(0.57 \mathrm{~mL}, 4.42 \mathrm{mmol})$ and $\mathrm{K}_{2} \mathrm{CO}_{3}(2.33 \mathrm{~g}, 16.9 \mathrm{mmol})$ in acetone $(42 \mathrm{~mL})$ gave $3 \mathrm{e}$ as a white solid (1.19 g, 78\%); $R_{f}=0.47$ (EtOAc $1: n$-hexane 3); ${ }^{1} \mathrm{H}$ NMR (400 $\left.\mathrm{MHz}, \mathrm{CDCl}_{3}\right) \delta$ 7.56-7.54 (m, 1 ArH), 7.40-7.38 (m, 1 ArH), 7.30-7.23 (m, 2 ArH), 7.11 (d, J=8.3 Hz, 2 ArH), 6.93 (d, $J=8.3 \mathrm{~Hz}, 2 \mathrm{ArH}), 5.14$ (s, OCH $), 4.55$ (br s, NH), 3.35-3.33 (m, $\mathrm{NHCH}_{2} \mathrm{CH}_{2}$ ), 2.73 $\left(\mathrm{t}, J=6.9 \mathrm{~Hz}, \mathrm{NHCH}_{2} \mathrm{CH}_{2}\right), 1.43\left(\mathrm{~s}, \mathrm{C}\left(\mathrm{CH}_{3}\right)_{3}\right) ;{ }^{13} \mathrm{C} \mathrm{NMR}\left(75 \mathrm{MHz}, \mathrm{CDCl}_{3}\right) \delta 157.2(\mathrm{ArC})$, $155.9(\mathbf{C}(\mathrm{O})), 134.9,132.6,132.2,131.6,129.8,129.4,129.0,128.8,127.0(\mathrm{ArC}), 79.2$

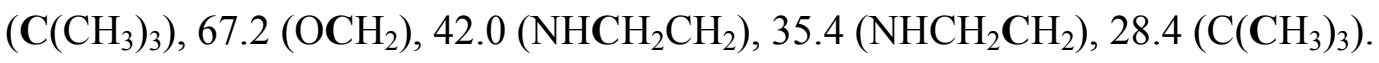

\section{tert-Butyl (4-((3-chlorobenzyl)oxy)phenethyl)carbamate (3f)}

Using Method A, N-(tert-butoxycarbonyl)tyramine (1.00 g, $4.21 \mathrm{mmol})$, 3-chlorobenzyl bromide $(0.58 \mathrm{~mL}, 4.64 \mathrm{mmol})$ and $\mathrm{K}_{2} \mathrm{CO}_{3}(2.33 \mathrm{~g}, 16.9 \mathrm{mmol})$ in acetone $(42 \mathrm{~mL})$ gave $\mathbf{3 f}$ as a white solid $(1.40 \mathrm{~g}, 92 \%) ; R_{f}=0.46($ EtOAc $1: n$-hexane 3$) ;{ }^{1} \mathrm{H}$ NMR $\left(400 \mathrm{MHz}, \mathrm{CDCl}_{3}\right) \delta$ 7.43 (s, 1 ArH), 7.34-7.26 (m, 3 ArH), 7.11 (d, $J=8.5$ Hz, 2 ArH), 6.92-6.87 (m, 2 ArH), 5.01 (s, $\mathrm{OCH}_{2}$ ), 4.53 (br s, NH), 3.35-3.33 (m, $\left.\mathrm{NHCH}_{2} \mathrm{CH}_{2}\right), 2.73$ (t, $J=6.9 \mathrm{~Hz}, \mathrm{NHCH}_{2} \mathrm{CH}_{2}$ ), 1.43 $\left(\mathrm{s}, \mathrm{C}\left(\mathrm{CH}_{3}\right)_{3}\right) ;{ }^{13} \mathrm{C} \mathrm{NMR}\left(75 \mathrm{MHz}, \mathrm{CDCl}_{3}\right) \delta 157.2(\mathrm{ArC}), 155.9(\mathbf{C}(\mathrm{O})), 139.3,134.5,131.7$, 129.8, 128.1, 127.4, 125.3, $115.0(\mathrm{ArC}), 79.2\left(\mathbf{C}\left(\mathrm{CH}_{3}\right)_{3}\right), 69.3\left(\mathrm{OCH}_{2}\right), 42.0\left(\mathrm{NHCH}_{2} \mathrm{CH}_{2}\right)$, $35.4\left(\mathrm{NHCH}_{2} \mathrm{CH}_{2}\right), 28.4\left(\mathrm{C}\left(\mathrm{CH}_{3}\right)_{3}\right)$. 


\section{tert-Butyl (4-((4-chlorobenzyl)oxy)phenethyl)carbamate (3g)}

Using Method A, N-(tert-butoxycarbonyl)tyramine (1.00 g, $4.21 \mathrm{mmol})$, 4-chlorobenzyl bromide $(0.95 \mathrm{~g}, 4.64 \mathrm{mmol})$ and $\mathrm{K}_{2} \mathrm{CO}_{3}(2.33 \mathrm{~g}, 16.9 \mathrm{mmol})$ in acetone $(42 \mathrm{~mL})$ gave $3 \mathrm{~g}$ as a white solid (1.40 g, 94\%); $R_{f}=0.36$ (EtOAc $1: n$-hexane 3$) ;{ }^{1} \mathrm{H}$ NMR $\left(400 \mathrm{MHz}, \mathrm{CDCl}_{3}\right) \delta$ 7.35 (s, 4 ArH), 7.10 (d, J=8.4 Hz, 2 ArH), 6.89 (d, J=8.6 Hz, 2 ArH), 5.00 (s, OCH ), 4.53 (br s, NH), 3.35-3.33 (m, $\left.\mathrm{NHCH}_{2} \mathrm{CH}_{2}\right), 2.73$ (t, $\left.J=6.9 \mathrm{~Hz}, \mathrm{NHCH}_{2} \mathrm{CH}_{2}\right), 1.43\left(\mathrm{~s}, \mathrm{C}\left(\mathrm{CH}_{3}\right)_{3}\right)$; ${ }^{13} \mathrm{C}$ NMR $\left(75 \mathrm{MHz}, \mathrm{CDCl}_{3}\right) \delta 157.2(\mathrm{ArC}), 155.9(\mathbf{C}(\mathrm{O})), 135.7,133.7,131.6,129.8,128.8$, 115.0 (ArC), $79.2\left(\mathbf{C}\left(\mathrm{CH}_{3}\right)_{3}\right), 69.3\left(\mathrm{OCH}_{2}\right), 42.0\left(\mathrm{NHCH}_{2} \mathrm{CH}_{2}\right), 35.4\left(\mathrm{NHCH}_{2} \mathrm{CH}_{2}\right), 28.4$ $\left(\mathrm{C}\left(\mathrm{CH}_{3}\right)_{3}\right)$.

\section{tert-Butyl (4-((2-(trifluoromethyl)oxy)phenethyl)carbamate (3h)}

Using Method A, N-(tert-butoxycarbonyl)tyramine $(1.00$ g, $4.21 \mathrm{mmol})$, 2(trifluoromethyl)benzyl bromide $(0.67 \mathrm{~mL}, 4.42 \mathrm{mmol})$ and $\mathrm{K}_{2} \mathrm{CO}_{3}(2.33 \mathrm{~g}, 16.9 \mathrm{mmol})$ in acetone $(42 \mathrm{~mL})$ gave $3 \mathbf{h}$ as a white solid $(1.26 \mathrm{~g}, 75 \%) ; R_{f}=0.46($ EtOAc $1: n$-hexane 3$) ;{ }^{1} \mathrm{H}$ NMR (400 MHz, $\left.\mathrm{CDCl}_{3}\right) \delta 7.75(\mathrm{~d}, J=7.7 \mathrm{~Hz}, 1 \mathrm{ArH}), 7.69$ (d, $\left.J=7.8 \mathrm{~Hz}, 1 \mathrm{ArH}\right), 7.56(\mathrm{t}, J$ $=7.6 \mathrm{~Hz}, 1 \mathrm{ArH}), 7.41$ (t, $J=7.6 \mathrm{~Hz}, 1 \mathrm{ArH}), 7.11(\mathrm{~d}, J=8.4 \mathrm{~Hz}, 2 \operatorname{ArH}), 6.91(\mathrm{~d}, J=8.4 \mathrm{~Hz}$, $2 \mathrm{ArH}), 5.25$ (s, $\left.\mathrm{OCH}_{2}\right), 4.54$ (br s, NH), 3.35-3.33 (m, $\left.\mathrm{NHCH}_{2} \mathrm{CH}_{2}\right), 2.73$ (t, $J=6.9 \mathrm{~Hz}$, $\left.\mathrm{NHCH}_{2} \mathrm{CH}_{2}\right), 1.43\left(\mathrm{~s}, \mathrm{C}\left(\mathrm{CH}_{3}\right)_{3}\right) ;{ }^{13} \mathrm{C} \mathrm{NMR}\left(75 \mathrm{MHz}, \mathrm{CDCl}_{3}\right) \delta 157.0(\mathrm{ArC}), 155.9(\mathbf{C}(\mathrm{O}))$, $135.9,132.2,131.8,129.9,128.6,127.7,127.3$ (q, $\left.J_{\mathrm{C}-\mathrm{F}}=30.7 \mathrm{~Hz}\right), 125.9$ (q, $\left.J_{\mathrm{C}-\mathrm{F}}=5.7 \mathrm{~Hz}\right)$, $119.4\left(\mathrm{q}, J_{\mathrm{C}-\mathrm{F}}=272.0 \mathrm{~Hz}, \mathbf{C F}_{3}\right), 115.0(\mathrm{ArC}), 79.2\left(\mathbf{C}\left(\mathrm{CH}_{3}\right)_{3}\right), 66.2\left(\mathrm{OCH}_{2}\right), 42.0\left(\mathrm{NHCH}_{2} \mathrm{CH}_{2}\right)$, $35.4\left(\mathrm{NHCH}_{2} \mathrm{CH}_{2}\right), 28.4\left(\mathrm{C}\left(\mathrm{CH}_{3}\right)_{3}\right)$.

\section{tert-Butyl (4-((3-(trifluoromethyl)oxy)phenethyl)carbamate (3i)}

Using Method A, N-(tert-butoxycarbonyl)tyramine $(1.00 \quad \mathrm{~g}, 4.21 \mathrm{mmol}), \quad 3-$ (trifluoromethyl)benzyl bromide $(0.71 \mathrm{~mL}, 4.64 \mathrm{mmol})$ and $\mathrm{K}_{2} \mathrm{CO}_{3}(2.33 \mathrm{~g}, 16.9 \mathrm{mmol})$ in acetone $(42 \mathrm{~mL})$ gave $3 \mathbf{i}$ as a white solid $(1.30 \mathrm{~g}, 78 \%) ; R_{f}=0.4($ EtOAc $1: \mathbf{n}$-hexane 3$) ;{ }^{1} \mathrm{H}$ $\operatorname{NMR}\left(300 \mathrm{MHz}, \mathrm{CDCl}_{3}\right) \delta 7.70$ (s, $\left.1 \mathrm{ArH}\right), 7.63-7.47$ (m, $\left.3 \mathrm{ArH}\right), 7.12$ (d, $\left.J=8.5 \mathrm{~Hz}, 2 \mathrm{ArH}\right)$, 6.92 (d, $J=8.6 \mathrm{~Hz}, 2 \mathrm{ArH}), 5.08$ (s, $\left.\mathrm{OCH}_{2}\right), 4.54$ (br s, NH), 3.35-3.33 (m, $\mathrm{NHCH}_{2} \mathrm{CH}_{2}$ ), 2.73 $\left(\mathrm{t}, J=6.9 \mathrm{~Hz}, \mathrm{NHCH}_{2} \mathrm{CH}_{2}\right), 1.43\left(\mathrm{~s}, \mathrm{C}\left(\mathrm{CH}_{3}\right)_{3}\right) ;{ }^{13} \mathrm{C} \mathrm{NMR}\left(75 \mathrm{MHz}, \mathrm{CDCl}_{3}\right) \delta 157.1$ (ArC), $155.9(\mathbf{C}(\mathrm{O})), 138.2,131.8,131.0\left(\mathrm{q}, J_{\mathrm{C}-\mathrm{F}}=32.2 \mathrm{~Hz}\right), 130.6,129.9,129.1,124.4\left(\mathrm{q}, J_{\mathrm{C}-\mathrm{F}}=46.7\right.$ $\mathrm{Hz}), 124.1\left(\mathrm{q}, J_{\mathrm{C}-\mathrm{F}}=270.7 \mathrm{~Hz}, \mathrm{CF}_{3}\right), 115.0(\mathrm{ArC}), 79.2\left(\mathbf{C}\left(\mathrm{CH}_{3}\right)_{3}\right), 69.3\left(\mathrm{OCH}_{2}\right), 42.0$

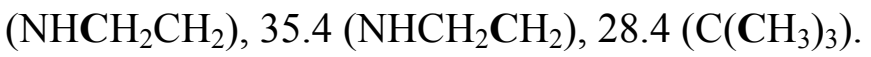

\section{tert-Butyl (4-((4-(trifluoromethyl)oxy)phenethyl)carbamate (3j)}

Using Method A, N-(tert-butoxycarbonyl)tyramine $(1.00 \quad \mathrm{~g}, \quad 4.21 \mathrm{mmol}), \quad 4-$ (trifluoromethyl)benzyl bromide $(0.78 \mathrm{~mL}, 5.06 \mathrm{mmol})$ and $\mathrm{K}_{2} \mathrm{CO}_{3}(2.33 \mathrm{~g}, 16.9 \mathrm{mmol})$ in 
acetone $(42 \mathrm{~mL})$ gave $3 \mathbf{j}$ as a white solid $(1.23 \mathrm{~g}, 73 \%) ; R_{f}=0.41$ (EtOAc $1: n$-hexane 3$) ;{ }^{1} \mathrm{H}$ $\operatorname{NMR}\left(300 \mathrm{MHz}, \mathrm{CDCl}_{3}\right) \delta 7.64(\mathrm{~d}, J=8.2 \mathrm{~Hz}, 2 \mathrm{ArH}) 7.55(\mathrm{~d}, J=8.0 \mathrm{~Hz}, 2 \mathrm{ArH}) 7.12(\mathrm{~d}, J$ $=8.5 \mathrm{~Hz}, 2 \mathrm{ArH})$ 6.93.63-6.89 (m, $2 \mathrm{ArH}), 5.11$ (s, OCH $), 4.53$ (br s, NH), 3.35-3.33 (m, $\left.\mathrm{NHCH}_{2} \mathrm{CH}_{2}\right), 2.73\left(\mathrm{t}, J=6.9 \mathrm{~Hz}, \mathrm{NHCH}_{2} \mathrm{CH}_{2}\right), 1.43\left(\mathrm{~s}, \mathrm{C}\left(\mathrm{CH}_{3}\right)_{3}\right) ;{ }^{13} \mathrm{C} \mathrm{NMR}\left(100 \mathrm{MHz}, \mathrm{CDCl}_{3}\right)$ $\delta 157.1(\operatorname{ArC}), 155.9(\mathbf{C}(\mathrm{O})), 141.2,131.8,130.1\left(\mathrm{q}, J_{\mathrm{C}-\mathrm{F}}=32.2 \mathrm{~Hz}\right), 129.9,127.4,125.5(\mathrm{q}$, $\left.J_{\mathrm{C}-\mathrm{F}}=3.8 \mathrm{~Hz}\right), 124.1\left(\mathrm{q}, J_{\mathrm{C}-\mathrm{F}}=270.4 \mathrm{~Hz}, \mathbf{C F}_{3}\right), 115.0(\mathrm{ArC}), 79.2\left(\mathbf{C}\left(\mathrm{CH}_{3}\right)_{3}\right), 69.2\left(\mathrm{OCH}_{2}\right)$, $42.0\left(\mathrm{NHCH}_{2} \mathrm{CH}_{2}\right), 35.3\left(\mathrm{NHCH}_{2} \mathrm{CH}_{2}\right), 28.4\left(\mathrm{C}\left(\mathrm{CH}_{3}\right)_{3}\right)$.

\section{tert-Butyl (4-((2,4-dichlorobenzyl)oxy)phenethyl)carbamate (3k)}

Using Method A, N-(tert-butoxycarbonyl)tyramine ( $0.60 \mathrm{~g}, 2.53 \mathrm{mmol}), 2$,4-dichlorobenzyl bromide $(0.64 \mathrm{~g}, 2.65 \mathrm{mmol})$ and $\mathrm{K}_{2} \mathrm{CO}_{3}(1.40 \mathrm{~g}, 10.1 \mathrm{mmol})$ in acetone $(25 \mathrm{~mL})$ gave $3 \mathrm{k}$ as a white solid $(0.97 \mathrm{~g}, 97 \%) ; R_{f}=0.56($ EtOAc $1: n$-hexane 3$) ;{ }^{1} \mathrm{H}$ NMR $\left(400 \mathrm{MHz}, \mathrm{CDCl}_{3}\right) \delta$ $7.50(\mathrm{~d}, J=8.3 \mathrm{~Hz}, 1 \operatorname{ArH}), 7.41$ (s, $1 \operatorname{ArH}), 7.27$ (d, $J=7.6 \mathrm{~Hz}, 1 \operatorname{ArH}), 7.12$ (d, $J=8.2 \mathrm{~Hz}$, $2 \operatorname{ArH}), 6.90(\mathrm{~d}, J=8.3 \mathrm{~Hz}, 2 \mathrm{ArH}), 5.10$ (s, OCH $)_{2}, 4.55$ (br s, NH), 3.35-3.33 (m, $\left.\mathrm{NHCH}_{2} \mathrm{CH}_{2}\right), 2.74\left(\mathrm{t}, J=6.8 \mathrm{~Hz}, \mathrm{NHCH}_{2} \mathrm{CH}_{2}\right), 1.43\left(\mathrm{~s}, \mathrm{C}\left(\mathrm{CH}_{3}\right)_{3}\right) ;{ }^{13} \mathrm{C} \mathrm{NMR}\left(75 \mathrm{MHz}, \mathrm{CDCl}_{3}\right)$ $\delta 157.0$ (ArC), $155.9(\mathbf{C}(\mathrm{O})), 134.1,133.6,133.1,131.9,129.9,129.6,129.2,127.3,115.0$

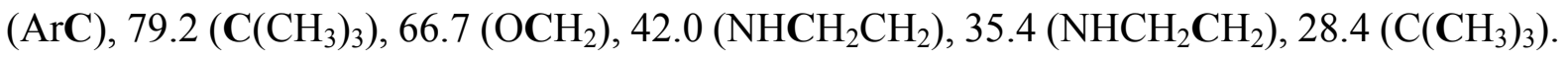

\section{tert-Butyl (4-((3,4-dichlorobenzyl)oxy)phenethyl)carbamate (3I)}

Using Method A, $N$-(tert-butoxycarbonyl)tyramine (1.00 g, $4.21 \mathrm{mmol})$, 3,4-dichlorobenzyl bromide $(0.64 \mathrm{~mL}, 4.42 \mathrm{mmol})$ and $\mathrm{K}_{2} \mathrm{CO}_{3}(2.33 \mathrm{~g}, 16.9 \mathrm{mmol})$ in acetone $(42 \mathrm{~mL})$ gave $3 \mathrm{l}$ as a white solid (1.60 g, 95\%); $R_{f}=0.38$ (EtOAc $1: n$-hexane 3); ${ }^{1} \mathrm{H}$ NMR $\left(400 \mathrm{MHz}, \mathrm{CDCl}_{3}\right) \delta$ 7.54-7.53 (m, 1 ArH), 7.45 (d, $J=8.2 \mathrm{~Hz}, 1 \mathrm{ArH}), 7.27-7.24$ (m, 1 ArH), 7.11 (d, $J=8.5 \mathrm{~Hz}$, 1 ArH), 6.90-6.87 (m, 2 ArH), 5.00 (s, OCH $), 4.53$ (br s, NH), 3.35-3.33 (m, $\mathrm{NHCH}_{2} \mathrm{CH}_{2}$ ), $2.74\left(\mathrm{t}, J=6.9 \mathrm{~Hz}, \mathrm{NHCH}_{2} \mathrm{CH}_{2}\right), 1.43\left(\mathrm{~s}, \mathrm{C}\left(\mathrm{CH}_{3}\right)_{3}\right) ;{ }^{13} \mathrm{C} \mathrm{NMR}\left(75 \mathrm{MHz}, \mathrm{CDCl}_{3}\right) \delta 157.0(\mathrm{ArC})$, $155.9(\mathbf{C}(\mathrm{O})), 137.5,132.8,132.0,130.6,129.9,129.2,126.5,115.0(\mathrm{ArC}), 79.3\left(\mathbf{C}\left(\mathrm{CH}_{3}\right)_{3}\right)$, $68.6\left(\mathrm{OCH}_{2}\right), 41.9\left(\mathrm{NHCH}_{2} \mathrm{CH}_{2}\right), 35.4\left(\mathrm{NHCH}_{2} \mathbf{C H}_{2}\right), 28.4\left(\mathrm{C}\left(\mathrm{CH}_{3}\right)_{3}\right)$.

\subsection{Synthesis of 4}

\section{2-(4-((3-Methoxybenzyl)oxy)phenyl)ethan-1-aminium chloride (4a)}

Using Method B, 3a (1.20 g, 3.36 mmol) and 4.0 M HCl in dioxane (5.04 mL, $20.2 \mathrm{mmol})$ gave 4a as a white solid (0.92 g, 93\%); $\mathrm{R}_{f}=0.00$ (EtOAc 9: acetone 1): $\mathrm{mp} 188.0-190.0{ }^{\circ} \mathrm{C}$; ${ }^{1} \mathrm{H}$ NMR (400 MHz, DMSO- $\left.d_{6}\right) \delta 8.16$ (s, NH $), 7.33-7.27$ (m, 1 ArH), 7.19-7.16 (m, 2 ArH), 7.01-6.87 (m, 5 ArH), $5.06\left(\mathrm{~s}, \mathrm{OCH}_{2}\right), 3.75\left(\mathrm{~s}, \mathrm{OCH}_{3}\right), 2.96-2.81\left(\mathrm{~m}, \mathrm{NH}_{3} \mathrm{CH}_{2} \mathrm{CH}_{2}\right) ;{ }^{13} \mathrm{C}$ NMR $\left(75 \mathrm{MHz}, \mathrm{DMSO}-d_{6}\right) \delta 159.3,157.1,138.7,129.7,129.5,119.6,114.9,113.1$ (ArC), 69.0 $\left(\mathrm{OCH}_{2}\right), 55.0\left(\mathrm{OCH}_{3}\right), 32.1\left(\mathrm{NCH}_{2} \mathrm{CH}_{2}\right)$. 


\section{2-(4-((2-Fluorobenzyl)oxy)phenyl)ethan-1-aminium chloride (4b)}

Using Method B, 3b $(0.90 \mathrm{~g}, 2.61 \mathrm{mmol})$ and $4.0 \mathrm{M} \mathrm{HCl}$ in dioxane $(3.91 \mathrm{~mL}, 15.6 \mathrm{mmol})$ gave $4 \mathbf{b}$ as a white solid $(0.73 \mathrm{~g}, 99 \%) ; \mathrm{R}_{f}=0.00$ (EtOAc 9: acetone 1): $\mathrm{mp}$ 201.0-202.0 ${ }^{\circ} \mathrm{C}$; ${ }^{1} \mathrm{H}$ NMR (400 MHz, DMSO- $d_{6}$ ) $\delta 8.24\left(\mathrm{~s}, \mathrm{NH}_{3}\right), 7.57-7.53(\mathrm{~m}, 6 \mathrm{ArH}), 7.00(\mathrm{~d}, J=8.5 \mathrm{~Hz}, 2$ ArH), 5.12 (s, OCH $\left.\mathbf{H}_{2}\right), 3.00-2.84\left(\mathrm{~m}, \mathrm{NH}_{3} \mathrm{CH}_{2} \mathrm{CH}_{2}\right) ;{ }^{13} \mathrm{C}$ NMR (75 MHz, DMSO- $\left.d_{6},\right) \delta 160.8$ $\left(\mathrm{d}, J_{\mathrm{C}-\mathrm{F}}=244.5 \mathrm{~Hz}\right), 157.5,131.1\left(\mathrm{~d}, J_{\mathrm{C}-\mathrm{F}}=4.0 \mathrm{~Hz}\right), 130.8\left(\mathrm{~d}, J_{\mathrm{C}-\mathrm{F}}=8.2 \mathrm{~Hz}\right), 130.3,130.2$, $125.0\left(\mathrm{~d}, J_{\mathrm{C}-\mathrm{F}}=3.4 \mathrm{~Hz}\right), 124.4\left(\mathrm{~d}, J_{\mathrm{C}-\mathrm{F}}=14.5 \mathrm{~Hz}\right), 115.8\left(\mathrm{~d}, J_{\mathrm{C}-\mathrm{F}}=21.0 \mathrm{~Hz}\right), 115.3(\mathrm{ArC}), 64.0$ $\left(\mathrm{OCH}_{2}\right), 32.5\left(\mathrm{NCH}_{2} \mathbf{C H}_{2}\right)$.

\section{2-(4-((3-Fluorobenzyl)oxy)phenyl)ethan-1-aminium chloride (4c)}

Using Method B, 3c (1.01 g, $2.93 \mathrm{mmol})$ and $4.0 \mathrm{M} \mathrm{HCl}$ in dioxane (4.39 mL, $17.6 \mathrm{mmol})$ gave 4c as a white solid (0.78 g, 94\%); $\mathrm{R}_{f}=0.00$ (EtOAc 9: acetone 1); ${ }^{1} \mathrm{H}$ NMR (400 MHz, DMSO$\left.d_{6},\right) \delta 8.08$ (s, NH $\left.\mathbf{N H}_{3}\right), 7.48-7.40$ (m, 1 ArH), 7.29-7.12 (m, 5 ArH), 6.99-6.96 (m, 2 ArH), 5.12 $\left(\mathrm{s}, \mathrm{OCH}_{2}\right), 3.00-2.80\left(\mathrm{~m}, \mathrm{NH}_{3} \mathrm{CH}_{2} \mathbf{C H}_{2}\right) ;{ }^{13} \mathrm{C} \mathrm{NMR}\left(75 \mathrm{MHz}, \mathrm{DMSO}-d_{6}\right) \delta 162.7\left(\mathrm{~d}, J_{\mathrm{C}-\mathrm{F}}=\right.$ $241.9 \mathrm{~Hz}), 157.4,140.6\left(\mathrm{~d}, J_{\mathrm{C}-\mathrm{F}}=7.3 \mathrm{~Hz}\right), 130.9\left(\mathrm{~d}, J_{\mathrm{C}-\mathrm{F}}=8.2 \mathrm{~Hz}\right), 130.2,130.1,123.9\left(\mathrm{~d}, J_{\mathrm{C}-}\right.$ $\left.\mathrm{F}_{\mathrm{F}}=2.6 \mathrm{~Hz}\right), 115.4,115.0\left(\mathrm{~d}, J_{\mathrm{C}-\mathrm{F}}=20.9 \mathrm{~Hz}\right), 114.6\left(\mathrm{~d}, J_{\mathrm{C}-\mathrm{F}}=21.6 \mathrm{~Hz}\right)(\mathrm{ArC}), 68.7\left(\mathrm{OCH}_{2}\right)$, $32.6\left(\mathrm{NCH}_{2} \mathrm{CH}_{2}\right)$.

\section{2-(4-((4-Fluorobenzyl)oxy)phenyl)ethan-1-aminium chloride (4d)}

Using Method B, 3d (1.30 g, $3.78 \mathrm{mmol})$ and 4.0 M HCl in dioxane (5.67 mL, $22.7 \mathrm{mmol})$ gave $4 \mathbf{d}$ as a white solid (1.02 g, 95\%); $\mathrm{R}_{f}=0.00$ (EtOAc 9: acetone 1); ${ }^{1} \mathrm{H}$ NMR (400 MHz, DMSO- $\left.d_{6}\right) \delta 8.17$ (s, NH NH$_{3}, 7.51-7.47$ (m, 2 ArH), 7.25-7.14 (m, 4 ArH), 6.98-6.92 (m, 2 ArH), $5.06\left(\mathrm{~s}, \mathrm{OCH}_{2}\right), 3.00-2.83\left(\mathrm{~m}, \mathrm{NH}_{3} \mathrm{CH}_{2} \mathrm{CH}_{2}\right) ;{ }^{13} \mathrm{C} \mathrm{NMR}\left(75 \mathrm{MHz}, \mathrm{DMSO}-d_{6}\right) \delta 162.2\left(\mathrm{~d}, J_{\mathrm{C}-\mathrm{F}}\right.$ $=241.8 \mathrm{~Hz}), 157.5,133.9,133.8,130.3\left(\mathrm{~d}, J_{\mathrm{C}-\mathrm{F}}=8.2 \mathrm{~Hz}\right), 130.2,130.1,115.7\left(\mathrm{~d}, J_{\mathrm{C}-\mathrm{F}}=21.2\right.$ $\mathrm{Hz}), 115.4(\mathrm{ArC}), 68.9\left(\mathrm{OCH}_{2}\right), 32.5\left(\mathrm{NCH}_{2} \mathrm{CH}_{2}\right)$.

\section{2-(4-((2-Chlorobenzyl)oxy)phenyl)ethan-1-aminium chloride (4e)}

Using Method B, 3e (1.15 g, $3.18 \mathrm{mmol})$ and $4.0 \mathrm{M} \mathrm{HCl}$ in dioxane $(4.77 \mathrm{~mL}, 19.1 \mathrm{mmol})$ gave 4e as a white solid (0.95 g, 99\%); $\mathrm{R}_{f}=0.00$ (EtOAc 9: acetone 1): $\mathrm{mp} 175.0-178.0{ }^{\circ} \mathrm{C} ;{ }^{1} \mathrm{H}$ NMR $\left(400 \mathrm{MHz}, \mathrm{DMSO}-d_{6}\right) \delta 8.26$ (s, NH NH$_{3}, 7.60-7.50$ (m, 2 ArH), 7.40-7.38 (m, 2 ArH), 7.21 (d, $J=8.2 \mathrm{~Hz}, 2 \mathrm{ArH}$ ), 7.00 (d, $J=8.3 \mathrm{~Hz}, 2 \mathrm{ArH}), 5.14$ (s, OCH $)_{2}, 3.00-2.85$ (m, $\mathrm{NH}_{3} \mathrm{CH}_{2} \mathrm{CH}_{2}$ ); ${ }^{13} \mathrm{C}$ NMR $\left(75 \mathrm{MHz}, \mathrm{DMSO}-d_{6}\right) \delta 157.5,134.8,133.0,130.5,130.4,130.3,130.2,129.8,128.9$, $127.8,115.3(\mathrm{ArC}), 67.3\left(\mathrm{OCH}_{2}\right), 32.5\left(\mathrm{NCH}_{2} \mathrm{CH}_{2}\right)$. 


\section{2-(4-((3-Chlorobenzyl)oxy)phenyl)ethan-1-aminium chloride (4f)}

Using Method B, 3f (1.35 g, 3.72 mmol) and 4.0 M HCl in dioxane (5.59 mL, $22.3 \mathrm{mmol})$ gave 4f as a white solid (1.08 g, 97\%); $\mathrm{R}_{f}=0.00$ (EtOAc 9: acetone 1); ${ }^{1} \mathrm{H}$ NMR (400 MHz, DMSO$\left.d_{6}\right) \delta 8.22\left(\mathrm{~s}, \mathrm{NH}_{3}\right), 7.51-7.41(\mathrm{~m}, 4 \mathrm{ArH}), 7.19(\mathrm{~d}, J=8.5 \mathrm{~Hz}, 2 \mathrm{ArH}), 6.97(\mathrm{~d}, J=8.5 \mathrm{~Hz}, 2$ ArH), $5.11\left(\mathrm{~s}, \mathrm{OCH}_{2}\right), 2.99-2.83\left(\mathrm{~m}, \mathrm{NH}_{3} \mathrm{CH}_{2} \mathrm{CH}_{2}\right) ;{ }^{13} \mathrm{C} \mathrm{NMR}\left(75 \mathrm{MHz}, \mathrm{DMSO}-d_{6}\right) \delta 157.4$, $140.3,133.6,130.8,130.3,130.2,128.1,127.6,126.5,115.4(\mathrm{ArC}), 68.7\left(\mathrm{OCH}_{2}\right), 32.5$ $\left(\mathrm{NCH}_{2} \mathrm{CH}_{2}\right)$.

\section{2-(4-((4-Chlorobenzyl)oxy)phenyl)ethan-1-aminium chloride (4g)}

Using Method B, 3g (1.38 g, $3.81 \mathrm{mmol})$ and $4.0 \mathrm{M} \mathrm{HCl}$ in dioxane $(5.72 \mathrm{~mL}, 22.9 \mathrm{mmol})$ gave $4 \mathrm{~g}$ as a white solid (1.06 g, 93\%); $\mathrm{R}_{f}=0.00$ (EtOAc 9: acetone 1); ${ }^{1} \mathrm{H}$ NMR (400 MHz, DMSO- $\left.d_{6}\right) \delta 8.16\left(\mathrm{~s}, \mathrm{NH}_{3}\right), 7.49-7.40(\mathrm{~m}, 4 \mathrm{ArH}), 7.18(\mathrm{~d}, J=8.5 \mathrm{~Hz}, 2 \mathrm{ArH}), 6.96(\mathrm{~d}, J=8.6$ $\mathrm{Hz}, 2 \mathrm{ArH}), 5.09$ (s, OCH $)$, 2.96-2.81 (m, $\left.\mathrm{NH}_{3} \mathrm{CH}_{2} \mathrm{CH}_{2}\right) ;{ }^{13} \mathrm{C}$ NMR (75 MHz, DMSO- $\left.d_{6}\right) \delta$ 161.8, 156.9, 136.2, 132.3, 129.7, 129.4, 128.4, 114.9 (ArC), $68.3\left(\mathrm{OCH}_{2}\right), 32.0\left(\mathrm{NCH}_{2} \mathrm{CH}_{2}\right)$.

\section{2-(4-((2-(Trifluoromethyl)benzyl)oxy)phenyl)ethan-1-aminium chloride (4h)}

Using Method B, 3h (1.20 g, $3.03 \mathrm{mmol})$ and 4.0 M HCl in dioxane (4.56 mL, $18.2 \mathrm{mmol})$ gave $4 \mathrm{~h}$ as a white solid $(0.99 \mathrm{~g}, 98 \%) ; \mathrm{R}_{f}=0.00$ (EtOAc $9:$ acetone 1$) ;{ }^{1} \mathrm{H}$ NMR $(400 \mathrm{MHz}$, DMSO- $\left.d_{6}\right) \delta 8.27$ (s, NH NH) $_{3}$ 7.81-7.57 (m, 4 ArH), 7.22 (d, $\left.J=8.4 \mathrm{~Hz}, 2 \mathrm{ArH}\right), 6.97$ (d, $J=8.4$ $\mathrm{Hz}, 2 \mathrm{ArH}), 5.22$ (s, OCH $), 3.00-2.86\left(\mathrm{~m}, \mathrm{NH}_{3} \mathrm{CH}_{2} \mathrm{CH}_{2}\right) ;{ }^{13} \mathrm{C}$ NMR $\left(75 \mathrm{MHz}, \mathrm{DMSO}-d_{6}\right) \delta$ $157.3,135.5,133.3,130.8,130.5,130.3,129.2,127.3$ (q, $\left.J_{\mathrm{C}-\mathrm{F}}=30.4 \mathrm{~Hz}\right), 126.6$ (q, $J_{\mathrm{C}-\mathrm{F}}=5.5$ $\mathrm{Hz}), 124.8\left(\mathrm{q}, J_{\mathrm{C}-\mathrm{F}}=272.3 \mathrm{~Hz}, \mathrm{CF}_{3}\right), 115.3(\mathrm{ArC}), 66.7\left(\mathrm{OCH}_{2}\right), 32.5\left(\mathrm{NCH}_{2} \mathbf{C H}_{2}\right)$.

\section{2-(4-((3-(Trifluoromethyl)benzyl)oxy)phenyl)ethan-1-aminium chloride (4i)}

Using Method B, 3i (1.25 g, 3.15 mmol) and 4.0 M HCl in dioxane (4.73 mL, $18.9 \mathrm{mmol})$ gave 4i as a white solid $(0.98 \mathrm{~g}, 93 \%) ; \mathrm{R}_{f}=0.00$ (EtOAc 9 : acetone 1$)$ ) ${ }^{1} \mathrm{H}$ NMR (300 MHz, DMSO$\left.d_{6}\right) \delta 8.10\left(\mathrm{~s}, \mathrm{NH}_{3}\right), 7.81-7.62(\mathrm{~m}, 4 \mathrm{ArH}), 7.20(\mathrm{~d}, J=8.6 \mathrm{~Hz}, 2 \mathrm{ArH}), 7.00(\mathrm{~d}, J=8.7 \mathrm{~Hz}, 2$ ArH), 5.20 (s, OCH $), 3.00-2.81\left(\mathrm{~m}, \mathrm{NH}_{3} \mathrm{CH}_{2} \mathrm{CH}_{2}\right) ;{ }^{13} \mathrm{C} \mathrm{NMR}\left(75 \mathrm{MHz}, \mathrm{DMSO}-d_{6}\right) \delta 157.0$, $138.8,131.7,129.9,129.7,129.3\left(\mathrm{q}, J_{\mathrm{C}-\mathrm{F}}=31.6 \mathrm{~Hz}\right), 124.6,124.3\left(\mathrm{q}, J_{\mathrm{C}-\mathrm{F}}=270.5 \mathrm{~Hz}, \mathrm{CF}_{3}\right)$, 124.0, 123.9, 115.1 (ArC), $68.4\left(\mathrm{OCH}_{2}\right), 32.2\left(\mathrm{NCH}_{2} \mathrm{CH}_{2}\right)$.

2-(4-((4-(Trifluoromethyl)benzyl)oxy)phenyl)ethan-1-aminium chloride (4j) 
Using Method B, 3j (1.17 g, $2.96 \mathrm{mmol})$ and $4.0 \mathrm{M} \mathrm{HCl}$ in dioxane (4.44 mL, $17.8 \mathrm{mmol})$ gave $4 \mathbf{j}$ as a white solid $(0.77 \mathrm{~g}, 78 \%) ; \mathrm{R}_{f}=0.00$ (EtOAc 9: acetone 1); ${ }^{1} \mathrm{H}$ NMR (300 MHz, DMSO$\left.d_{6}\right) \delta 8.10\left(\mathrm{~s}, \mathrm{NH}_{3}\right), 7.76(\mathrm{~d}, J=8.2 \mathrm{~Hz}, 2 \mathrm{ArH}), 7.66(\mathrm{~d}, J=8.1 \mathrm{~Hz}, 2 \mathrm{ArH}), 7.19(\mathrm{~d}, J=8.5$ $\mathrm{Hz}, 2 \mathrm{ArH}), 6.98$ (d, $J=8.5 \mathrm{~Hz}, 2 \mathrm{ArH}), 5.22\left(\mathrm{~s}, \mathrm{OCH}_{2}\right), 3.00-2.80\left(\mathrm{~m}, \mathrm{NH}_{3} \mathrm{CH}_{2} \mathrm{CH}_{2}\right) ;{ }^{13} \mathrm{C}$ NMR (100 MHz, DMSO- $\left.d_{6}\right) \delta 157.3,142.6,130.3,130.2,128.7$ (q, $\left.J_{\mathrm{C}-\mathrm{F}}=31.5 \mathrm{~Hz}\right), 128.4$, $125.8\left(\mathrm{q}, J_{\mathrm{C}-\mathrm{F}}=3.9 \mathrm{~Hz}\right), 124.7\left(\mathrm{q}, J_{\mathrm{C}-\mathrm{F}}=270.4 \mathrm{~Hz}, \mathrm{CF}_{3}\right), 115.4(\mathrm{ArC}), 68.7\left(\mathrm{OCH}_{2}\right), 32.5$ $\left(\mathrm{NCH}_{2} \mathrm{CH}_{2}\right)$.

\section{2-(4-((2,4-Dichlorobenzyl)oxy)phenyl)ethan-1-aminium chloride (4k)}

Using Method B, 3k (0.95 g, $2.40 \mathrm{mmol})$ and $4.0 \mathrm{M} \mathrm{HCl}$ in dioxane $(3.60 \mathrm{~mL}, 14.4 \mathrm{mmol})$

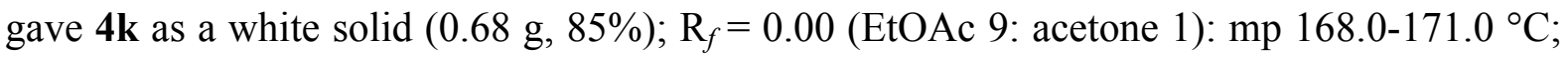
${ }^{1} \mathrm{H}$ NMR (400 MHz, DMSO- $\left.d_{6}\right) \delta 8.23$ (s, NH $), 7.69-7.47$ (m, $\left.3 \mathrm{ArH}\right), 7.21(\mathrm{~d}, J=8.5 \mathrm{~Hz}, 2$ $\operatorname{ArH}), 6.99(\mathrm{~d}, J=8.5 \mathrm{~Hz}, 2 \mathrm{ArH}), 5.12\left(\mathrm{~s}, \mathrm{OCH}_{2}\right), 3.00-2.85\left(\mathrm{~m}, \mathrm{NH}_{3} \mathrm{CH}_{2} \mathrm{CH}_{2}\right) ;{ }^{13} \mathrm{C} \mathrm{NMR}(75$ $\left.\mathrm{MHz}, \mathrm{DMSO}-d_{6}\right) \delta 157.3,134.1,134.0,133.9,131.7,130.5,130.3,129.4,128.0,115.4(\mathrm{ArC})$, $66.8\left(\mathrm{OCH}_{2}\right), 32.5\left(\mathrm{NCH}_{2} \mathrm{CH}_{2}\right)$.

\section{2-(4-((3,4-Dichlorobenzyl)oxy)phenyl)ethan-1-aminium chloride (4l)}

Using Method B, 31 (1.54 g, $3.90 \mathrm{mmol})$ and $4.0 \mathrm{M} \mathrm{HCl}$ in dioxane $(5.85 \mathrm{~mL}, 23.4 \mathrm{mmol})$ gave $4 \mathrm{l}$ as a white solid (1.26 g, 97\%); $\mathrm{R}_{f}=0.00$ (EtOAc 9: acetone 1): $\mathrm{mp}$ 191.0-193.0 ${ }^{\circ} \mathrm{C} ;{ }^{1} \mathrm{H} \mathrm{NMR}$ $\left(300 \mathrm{MHz}, \mathrm{DMSO}-d_{6}\right) \delta 8.14\left(\mathrm{~s}, \mathrm{NH}_{3}\right), 7.72-7.65$ (m, 2 ArH), 7.46-7.42 (m, 1 ArH), 7.19 (d, $J=8.6 \mathrm{~Hz}, 2 \mathrm{ArH}), 6.97(\mathrm{~d}, J=8.6 \mathrm{~Hz}, 2 \mathrm{ArH}), 5.11\left(\mathrm{~s}, \mathrm{OCH}_{2}\right), 3.00-2.81\left(\mathrm{~m}, \mathrm{NH}_{3} \mathrm{CH}_{2} \mathrm{CH}_{2}\right)$; ${ }^{13} \mathrm{C}$ NMR $\left(75 \mathrm{MHz}, \mathrm{DMSO}-d_{6}\right) \delta 157.2,139.0,131.6,131.2,130.7,130.3,130.2,129.8,128.2$, $115.4(\mathrm{ArC}), 68.1\left(\mathrm{OCH}_{2}\right), 32.6\left(\mathrm{NCH}_{2} \mathrm{CH}_{2}\right)$.

Scheme S2. Synthetic route for 6.

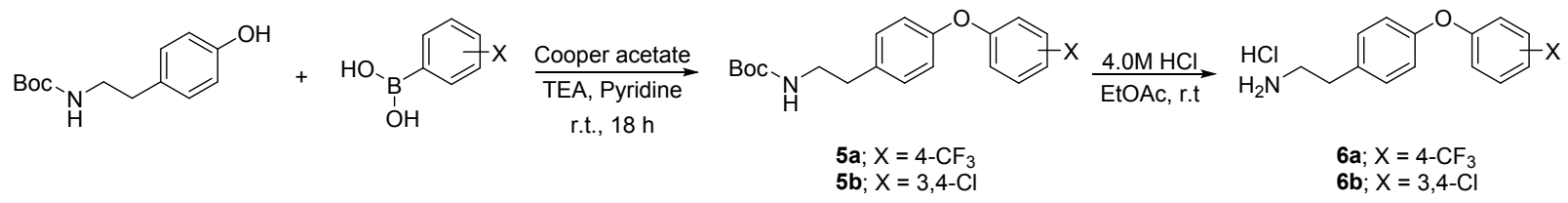

\subsection{Synthesis of 5}

tert-Butyl (4-(4-(trifluoromethyl)phenoxy)phenethyl)carbamate (5a)

Using Method C, $N$-boc tyramine (1.00 g, $4.21 \mathrm{mmol}$ ), (4-(trifluoromethyl)phenyl)boronic acid (1.60 g, $8.43 \mathrm{mmol})$, Copper(II) acetate $(0.38 \mathrm{~g}, 2.13 \mathrm{mmol})$, triethylamine $(2.93 \mathrm{~mL}, 21.1$ $\mathrm{mmol})$ and pyridine $(1.70 \mathrm{~mL}, 21.1 \mathrm{mmol})$ gave $4 \mathbf{a}$ as a white solid $(0.36 \mathrm{~g}, 22 \%) ; R_{f}=0.40$ (n-hexane 5: EtOAc 1); ${ }^{1} \mathrm{H}$ NMR (300 MHz, $\left.\mathrm{CDCl}_{3}\right) \delta 7.56(\mathrm{~d}, J=8.6 \mathrm{~Hz}, 2 \mathrm{ArH}), 7.21(\mathrm{~d}, J$ 
$=8.4 \mathrm{~Hz}, 2 \mathrm{ArH}), 6.98-7.04(\mathrm{~m}, 4 \mathrm{ArH}), 4.61$ (br s, C(O)ONH), 3.39 (q, $J=6.5 \mathrm{~Hz}$, $\left.\mathrm{NHCH}_{2} \mathrm{CH}_{2}\right), 2.80$ (t, $\left.J=7.0 \mathrm{~Hz}, \mathrm{NHCH}_{2} \mathrm{CH}_{2}\right), 1.44\left(\mathrm{~s}, \mathrm{C}\left(\mathrm{CH}_{3}\right)_{3}\right) ;{ }^{13} \mathrm{C} \mathrm{NMR}\left(75 \mathrm{MHz}, \mathrm{CDCl}_{3}\right)$ $\delta 160.7(\mathrm{ArC}), 155.9(\mathbf{C}(\mathrm{O})), 154.2,135.3,130.4,129.6,127.1\left(\mathrm{q}, J_{\mathrm{C}-\mathrm{F}}=3.8 \mathrm{~Hz}\right), 124.8\left(\mathrm{q}, J_{\mathrm{C}-\mathrm{F}}\right.$ $=32.5 \mathrm{~Hz}), 124.2\left(\mathrm{q}, J_{\mathrm{C}-\mathrm{F}}=269.6 \mathrm{~Hz}, \mathbf{C F}_{3}\right), 120.1,117.7(\mathrm{ArC}), 79.3\left(\mathbf{C}\left(\mathrm{CH}_{3}\right)_{3}\right), 41.9$

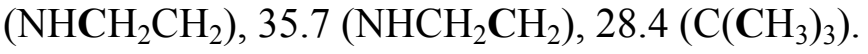

\section{tert-Butyl (4-(3,4-dichlorophenoxy)phenethyl)carbamate (5b)}

Using Method C, $N$-boc tyramine (3.00 g, $12.6 \mathrm{mmol})$, (3,4-dichlorophenyl) boronic acid (4.82 g, $25.3 \mathrm{mmol})$, Copper(II) acetate $(2.32 \mathrm{~g}, 12.8 \mathrm{mmol})$, triethylamine $(8.81 \mathrm{~mL}, 63.2 \mathrm{mmol})$ and pyridine $(5.11 \mathrm{~mL}, 63.2 \mathrm{mmol})$ gave $4 \mathbf{b}$ as a white solid $(0.69 \mathrm{~g}, 14 \%) ; R_{f}=0.51$ ( $n$-hexane 5: EtOAc 1); ${ }^{1} \mathrm{H}$ NMR $\left(300 \mathrm{MHz}, \mathrm{CDCl}_{3}\right) \delta 7.36(\mathrm{~d}, J=8.2 \mathrm{~Hz}, 1 \mathrm{ArH}), 7.18-7.21$ (m, $\left.2 \mathrm{ArH}\right)$, 7.06 (d, $J=2.8 \mathrm{~Hz}, 1$ ArH), 6.82-6.98 (m, 3 ArH), 4.58 (br s, C(O)ONH), 3.38 (q, $J=6.5 \mathrm{~Hz}$, $\left.\mathrm{NHCH}_{2} \mathrm{CH}_{2}\right), 2.79$ (t, $\left.J=7.0 \mathrm{~Hz}, \mathrm{NHCH}_{2} \mathrm{CH}_{2}\right), 1.44\left(\mathrm{~s}, \mathrm{C}\left(\mathrm{CH}_{3}\right)_{3}\right) ;{ }^{13} \mathrm{C} \mathrm{NMR}\left(75 \mathrm{MHz}, \mathrm{CDCl}_{3}\right)$ $\delta 156.8$ (ArC), $155.9(\mathbf{C}(\mathrm{O})), 154.6,135.1,133.2,131.0,130.4,126.3,120.1,119.6,117.8$ (ArC), $79.4\left(\mathbf{C}\left(\mathrm{CH}_{3}\right)_{3}\right), 41.8\left(\mathrm{NHCH}_{2} \mathrm{CH}_{2}\right), 35.6\left(\mathrm{NHCH}_{2} \mathrm{CH}_{2}\right), 28.4\left(\mathrm{C}\left(\mathrm{CH}_{3}\right)_{3}\right)$.

\subsection{Synthesis of 6}

\section{2-(4-(4-(Trifluoromethyl)phenoxy)phenyl)ethan-1-amine hyd rochloride (6a)}

Using Method B, 5a $(0.33 \mathrm{~g}, 0.87 \mathrm{mmol})$ and $4.0 \mathrm{M} \mathrm{HCl}$ in dioxane $(0.65 \mathrm{~mL}, 2.60 \mathrm{mmol})$ gave 6a as a white solid $(0.17 \mathrm{~g}, 60 \%) ; R_{f}=0.00$ (EtOAc 9: acetone 1$) ;{ }^{1} \mathrm{H}$ NMR $(300 \mathrm{MHz}$, DMSO- $\left.d_{6},\right) \delta 8.31$ (br s, NH NH$_{3}, 7.73(\mathrm{~d}, J=8.6 \mathrm{~Hz}, 2 \mathrm{ArH}), 7.37$ (d, $\left.J=8.5 \mathrm{~Hz}, 2 \mathrm{ArH}\right), 7.09$ 7.15 (m, 4 ArH), 2.94-3.09 (m, $\mathrm{NH}_{3} \mathrm{CH}_{2} \mathrm{CH}_{2}$ ); ${ }^{13} \mathrm{C}$ NMR (75 MHz, DMSO- $\left.d_{6}\right) \delta 161.1(\mathbf{C}(\mathrm{O})$ ), 154.1, 134.5, 131.1, $127.8\left(\mathrm{q}, J_{\mathrm{C}-\mathrm{F}}=3.8 \mathrm{~Hz}\right), 124.8\left(\mathrm{q}, J_{\mathrm{C}-\mathrm{F}}=269.6 \mathrm{~Hz}, \mathbf{C F}_{3}\right), 123.6\left(\mathrm{q}, J_{\mathrm{C}-\mathrm{F}}=\right.$ $32.0 \mathrm{~Hz}), 120.7,118.2$ (ArC), $40.3\left(\mathrm{NH}_{3} \mathrm{CH}_{2} \mathrm{CH}_{2}\right), 32.6\left(\mathrm{NH}_{3} \mathrm{CH}_{2} \mathrm{CH}_{2}\right)$.

\section{2-(4-(3,4-Dichlorophenoxy)phenyl)ethan-1-amine hydrochlori de (6b)}

Using Method B, 5b $(0.68 \mathrm{~g}, 1.78 \mathrm{mmol})$ and $4.0 \mathrm{M} \mathrm{HCl}$ in dioxane $(1.33 \mathrm{~mL}, 5.34 \mathrm{mmol})$ gave $6 \mathbf{b}$ as a white solid $(0.48 \mathrm{~g}, 57 \%) ; R_{f}=0.00$ (EtOAc 9: acetone 1); ${ }^{1} \mathrm{H}$ NMR $(300 \mathrm{MHz}$, DMSO- $d_{6}$, ) $\delta 8.26$ (br s, NH NH) 7.62 (d, $J=8.9$ Hz, 1 ArH), 6.97-7.36 (m, 4 ArH), 2.91-3.03 $\left(\mathrm{m}, \mathrm{NH}_{3} \mathrm{CH}_{2} \mathrm{CH}_{2}\right) ;{ }^{13} \mathrm{C} \mathrm{NMR}\left(75 \mathrm{MHz}, \mathrm{DMSO}-d_{6}\right) \delta 157.2(\mathbf{C}(\mathrm{O})), 154.6,134.5,134.2,132.4$, 132.0, 131.1, 125.5, 120.2, 120.1, 118.8 (ArC), $40.8\left(\mathrm{NH}_{3} \mathrm{CH}_{2} \mathrm{CH}_{2}\right), 32.6\left(\mathrm{NH}_{3} \mathrm{CH}_{2} \mathrm{CH}_{2}\right)$.

Scheme S3. Synthetic route for 8 .

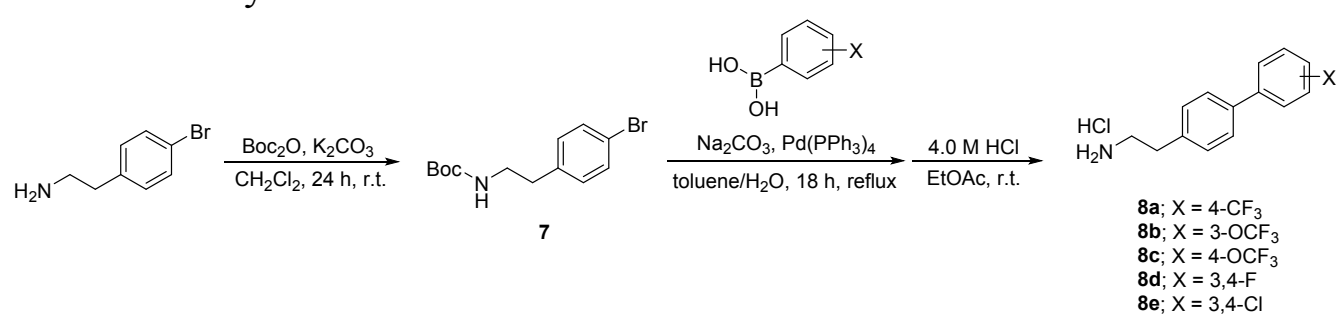




\subsection{Synthesis of 7}

\section{tert-Butyl (4-bromophenethyl)carbamate (7)}

Using Method D, 2-(4-bromophenyl)ethan-1-amine $(4.00 \mathrm{~mL}, 25.8 \mathrm{mmol}$ ), potassium carbonate $(5.35 \mathrm{~g}, 38.7 \mathrm{mmol})$ and di-tert-butyl dicarbonate $(7.40 \mathrm{~mL}, 27.1 \mathrm{mmol})$ gave 6 as a white solid (7.22 g, 93\%); $R_{f}=0.37$ ( $n$-hexane $5:$ EtOAc 1$) ;{ }^{1} \mathrm{H}$ NMR (300 MHz, DMSO- $d_{6}$ ) $\delta 7.42(\mathrm{~d}, J=8.2 \mathrm{~Hz}, 2 \mathrm{ArH}), 7.06(\mathrm{~d}, J=8.0 \mathrm{~Hz}, 2 \mathrm{ArH}), 3.34-3.35\left(\mathrm{~m}, \mathrm{NHCH}_{2} \mathrm{CH}_{2}\right), 2.75$ $\left(\mathrm{t}, J=6.6 \mathrm{~Hz}, \mathrm{NHCH}_{2} \mathrm{CH}_{2}\right), 1.43\left(\mathrm{~s}, \mathrm{C}\left(\mathrm{CH}_{3}\right)_{3}\right) ;{ }^{13} \mathrm{C} \mathrm{NMR}\left(75 \mathrm{MHz}\right.$, DMSO- $\left.d_{6}\right) \delta 155.8(\mathbf{C}(\mathrm{O}))$, 138.0, 131.6, 130.6, $120.2(\mathrm{ArC}), 79.3\left(\mathbf{C}\left(\mathrm{CH}_{3}\right)_{3}\right), 41.6\left(\mathrm{NHCH}_{2} \mathrm{CH}_{2}\right), 35.7\left(\mathrm{NHCH}_{2} \mathbf{C H}_{2}\right), 28.4$ $\left(\mathrm{C}\left(\mathrm{CH}_{3}\right)_{3}\right)$.

\subsection{Synthesis of 8}

\section{2-(4'-(Trifluoromethyl)-[1,1'-biphenyl]-4-yl)ethan-1-amine hydrochloride (8a)}

Using Method B, 6 (2.78 g, 9.26 mmol), (4-(trifluoromethyl)phen yl)boronic acid (2.11 g, 11.1 $\mathrm{mmol}), \mathrm{Na}_{2} \mathrm{CO}_{3}(4.91 \mathrm{~g}, 46.3 \mathrm{mmol})$ and tetrakis(trip henylphosphine)palladium(0) $(0.43 \mathrm{~g}$, $0.37 \mathrm{mmol})$, followed by $4.0 \mathrm{M} \mathrm{HCl}$ in dioxane $(11.0 \mathrm{~mL}, 43.9 \mathrm{mmol})$ gave $8 \mathbf{a}$ as a white solid $(2.18 \mathrm{~g}, 99 \%) ; R_{f}=0.00$ (EtOAc $9:$ acet one 1$) ;{ }^{1} \mathrm{H}$ NMR $\left(400 \mathrm{MHz}\right.$, DMSO- $\left.d_{6}\right) \delta 8.37$ (s, $\left.\mathrm{NH}_{3}\right), 7.91-7.71(\mathrm{~m}, 6 \mathrm{ArH}), 7.44(\mathrm{~d}, J=8.1 \mathrm{~Hz}, 2 \mathrm{ArH}), 3.11-3.01\left(\mathrm{~m}, \mathrm{NH}_{3} \mathrm{CH}_{2} \mathrm{CH}_{2}\right) ;{ }^{13} \mathrm{C}$ NMR $\left(75 \mathrm{MHz}\right.$, DMSO- $\left.d_{6}\right) \delta 144.3,138.4,137.4,129.9,128.2\left(\mathrm{q}, J_{\mathrm{C}-\mathrm{F}}=31.7 \mathrm{~Hz}\right), 127.7,127.6$, $126.2\left(\mathrm{q}, J_{\mathrm{C}-\mathrm{F}}=3.8 \mathrm{~Hz}\right), 124.8\left(\mathrm{q}, J_{\mathrm{C}-\mathrm{F}}=270.1 \mathrm{~Hz}, \mathbf{C F}_{3}\right)(\mathrm{ArC}), 33.0\left(\mathrm{NCH}_{2} \mathrm{CH}_{2}\right)$.

\section{2-(3'-(Trifluoromethoxy)-[1,1'-biphenyl]-4-yl)ethan-1-amine hydrochloride (8b)}

Using Method B, 6 (1.50 g, $5.00 \mathrm{mmol})$, 3-(trifluoromethoxy)phenylboronic acid (1.23 g, 6.00 mmol), tetrakis(triphenylphosphine)palladium(0) $(0.23 \mathrm{~g}, 0.20 \mathrm{mmol})$ and $\mathrm{Na}_{2} \mathrm{CO}_{3}(2.65 \mathrm{~g}$, $25.0 \mathrm{mmol})$ in toluene $/ \mathrm{H}_{2} \mathrm{O}(50 \mathrm{~mL} / 20 \mathrm{~mL})$, followed by $4.0 \mathrm{M} \mathrm{HCl}$ in dioxane $(3.75 \mathrm{~mL}, 15.0$ mmol) gave $8 \mathbf{b}$ as a white solid (1.07 g, 67\%); $\mathrm{R}_{f}=0.00$ (EtOAc 9 : acetone 1); mp 190.0$192.0{ }^{\circ} \mathrm{C} ;{ }^{1} \mathrm{H}$ NMR $\left(400 \mathrm{MHz}\right.$, DMSO- $\left.d_{6}\right) \delta 8.32\left(\mathrm{~s}, \mathrm{NH}_{3}\right), 7.74-7.35$ (m, $\left.8 \mathrm{ArH}\right), 3.10-3.02$ (m, $\mathrm{NH}_{3} \mathrm{CH}_{2} \mathrm{CH}_{2}$ ); ${ }^{13} \mathrm{C}$ NMR (75 MHz, DMSO- $d_{6}$ ) $\delta 149.5,142.7,138.1,137.3,131.3,129.9$, $129.0,127.5,126.1,120.6$ (q, $\left.J_{\mathrm{C}-\mathrm{F}}=254.6 \mathrm{~Hz}, \mathbf{C F}_{3}\right), 120.1,119.5(\mathrm{ArC}), 33.0\left(\mathrm{NCH}_{2} \mathbf{C H}_{2}\right)$.

\section{2-(4'-(Trifluoromethoxy)-[1,1'-biphenyl]-4-yl)ethan-1-amine hydrochloride (8c)}

Using Method B, 6 (4.00 g, $13.3 \mathrm{mmol})$, (4-(trifluoromethoxy)phenyl)boronic acid (3.29 g, $16.0 \mathrm{mmol}), \mathrm{Na}_{2} \mathrm{CO}_{3}(7.06 \mathrm{~g}, 66.6 \mathrm{mmol})$ and tetrakis $(\mathrm{t}$ riphenylphosphine)palladium $(0)(0.62$ $\mathrm{g}, 0.53 \mathrm{mmol})$, followed by $4.0 \mathrm{M} \mathrm{HCl}$ in dioxane $(11.0 \mathrm{~mL}, 43.9 \mathrm{mmol})$ gave $8 \mathrm{c}$ as a white solid ( $2.58 \mathrm{~g}, 97 \%$ ); $R_{f}=0.00$ (EtOAc $9:$ acet one 1 ); ${ }^{1} \mathrm{H}$ NMR (400 MHz, DMSO- $\left.d_{6}\right) \delta 8.31$ (s, $\left.\mathrm{NH}_{3}\right), 7.79$ (d, $\left.J=8.7 \mathrm{~Hz}, 2 \mathrm{ArH}\right), 7.66(\mathrm{~d}, J=8.1 \mathrm{~Hz}, 2 \mathrm{ArH}), 7.45$ (d, $J=8.2 \mathrm{~Hz}, 2 \mathrm{ArH}$ ), $7.40(\mathrm{~d}, J=8.1 \mathrm{~Hz}, 2 \mathrm{ArH}), 2.97-3.10\left(\mathrm{~m}, \mathrm{NH}_{3} \mathrm{CH}_{2} \mathrm{CH}_{2}\right) ;{ }^{13} \mathrm{C}$ NMR $\left(75 \mathrm{MHz}\right.$, DMSO- $\left.d_{6}\right) \delta$ $148.2,139.7,137.7,137.6,129.8,128.9,127.5,121.9,120.6\left(\mathrm{q}, J_{\mathrm{C}-\mathrm{F}}=254.6 \mathrm{~Hz}, \mathrm{CF}_{3}\right)(\mathrm{ArC})$, $33.0\left(\mathrm{NCH}_{2} \mathrm{CH}_{2}\right)$. 


\section{2-(3',4'-Difluoro-[1,1'-biphenyl]-4-yl)ethan-1-amine hydrochloride (8d)}

Using Method B, 6 (6.34 g, $21.1 \mathrm{mmol}$ ), (3,4-difluorophenyl)boronic acid (5.00 g, $31.7 \mathrm{mmol}$ ), $\mathrm{Na}_{2} \mathrm{CO}_{3}$ (11.2 g, $105.5 \mathrm{mmol}$ ) and tetrakis(triphenylphosph ine)palladium(0) (0.98 g, 0.84 $\mathrm{mmol}$ ), followed by $4.0 \mathrm{M} \mathrm{HCl}$ in dioxane $(18.5 \mathrm{~mL}, 74.0 \mathrm{mmol}$ ) gave $\mathbf{8 d}$ as a white solid (3.06 g, 92\%); $R_{f}=0.00$ (EtOAc $9:$ acetone 1); ${ }^{1} \mathrm{H}$ NMR (400 $\left.\mathrm{MHz}, \mathrm{CDCl}_{3}\right) \delta 7.95$ (br s, $\mathrm{NH}_{3}$ ), 7.74-7.79 (m, 1 ArH), 7.67 (d, $J=8.1 \mathrm{~Hz}, 2 \mathrm{ArH}), 7.48-7.54(\mathrm{~m}, 2 \mathrm{ArH}), 7.37$ (d, $J=8.1 \mathrm{~Hz}$, $2 \mathrm{ArH}), 3.08\left(\mathrm{~d}, J=7.8 \mathrm{~Hz}, \mathrm{NH}_{3} \mathrm{CH}_{2} \mathrm{CH}_{2}\right), 2.92$ (t, $J=7.8 \mathrm{~Hz}, \mathrm{NH}_{3} \mathrm{CH}_{2} \mathrm{CH}_{2}$ ).

\section{2-(3',4'-Dichloro-[1,1'-biphenyl]-4-yl)ethan-1-amine hydrochloride (8e)}

Using Method B, 6 (3.00 g, $9.99 \mathrm{mmol}$ ), (3,4-dichlorophenyl)boronic acid (2.29 g, $12.0 \mathrm{mmol}$ ), $\mathrm{Na}_{2} \mathrm{CO}_{3}(5.30 \mathrm{~g}, 50.0 \mathrm{mmol})$ and tetrakis(triphenylphosphi ne)palladium(0) (0.46 g, 0.40 $\mathrm{mmol})$, followed by $4.0 \mathrm{M} \mathrm{HCl}$ in dioxane $(9.20 \mathrm{~mL}, 36.8 \mathrm{mmol}$ ) gave $8 \mathbf{e}$ as a pale yellow solid $(1.77 \mathrm{~g}, 96 \%) ; R_{f}=0.00$ (EtOAc 9 : aceton e 1$) ;{ }^{1} \mathrm{H}$ NMR $\left(300 \mathrm{MHz}, \mathrm{DMSO}-d_{6}\right) \delta 8.06(\mathrm{~s}$, $\mathrm{NH}_{3}$ ), 7.93 (d, $\left.J=2.2 \mathrm{~Hz}, 1 \mathrm{ArH}\right), 7.69-7.73$ (m, 4 ArH), 7.38 (d, $\left.J=10.8 \mathrm{~Hz}, 2 \mathrm{ArH}\right), 3.05-$ $3.10\left(\mathrm{~m}, \mathrm{NH}_{3} \mathrm{CH}_{2} \mathrm{CH}_{2}\right), 2.91-2.97\left(\mathrm{~m}, \mathrm{NH}_{3} \mathrm{CH}_{2} \mathrm{CH}_{2}\right) ;{ }^{13} \mathrm{C} \mathrm{NMR}\left(75 \mathrm{MHz}, \mathrm{DMSO}-d_{6}\right) \delta 140.9$, $138.3,136.3,132.2,131.5,130.5,129.9,128.7,127.4,127.2(\mathrm{ArC}), 33.0\left(\mathrm{NCH}_{2} \mathrm{CH}_{2}\right)$.

\subsection{Synthesis of 9}

\section{2-((4-((3-Methoxybenzyl)oxy)phenethyl)amino)propanamide (9a)}

Using Method F, to a solution of $4 \mathbf{a}(0.85 \mathrm{~g}, 2.90 \mathrm{mmol}), \mathrm{K}_{2} \mathrm{CO}_{3}(1.60 \mathrm{~g}, 11.60 \mathrm{mmol})$, 2Bromopropionamide $(0.53 \mathrm{~g}, 3.50 \mathrm{mmol})$ in $\mathrm{CH}_{3} \mathrm{CN}(29 \mathrm{~mL})$ gave 9a as a white solid $(0.87 \mathrm{~g}$, $90 \%) ; R_{f}=0.33\left(\mathrm{CH}_{2} \mathrm{Cl}_{2} 9: \mathrm{MeOH} 1\right) ;{ }^{1} \mathrm{H} \mathrm{NMR}\left(400 \mathrm{MHz}, \mathrm{CDCl}_{3}\right) \delta$ 7.32-7.26 (m, $\left.1 \mathrm{ArH}\right)$, 7.12-7.09 (m, 2 ArH), 7.01-6.98 (m, 2 ArH), 6.93-6.84 (m, 3 ArH, C(O)NHH'), 5.40 (br s, $\mathrm{C}(\mathrm{O}) \mathrm{NHH}$ ), 5.03 (s, OCH $\mathbf{O H}_{2}, 3.82\left(\mathrm{~s}, \mathrm{OCH}_{3}\right) 3.15$ (q, $\left.J=7.0 \mathrm{~Hz}, \mathrm{CH}\right)$ 2.93-2.68 (m, $\left.\mathrm{NHCH}_{\mathbf{2}} \mathrm{CH}_{2}\right), 1.27\left(\mathrm{~d}, J=7.0 \mathrm{~Hz}, \mathrm{CH}_{3}\right) ;{ }^{13} \mathrm{C} \mathrm{NMR}\left(100 \mathrm{MHz}, \mathrm{CDCl}_{3}\right) \delta 178.0(\mathbf{C}(\mathrm{O})), 159.9$, 157.4, 138.8, 132.0, 129.7, 129.6, 119.6, 115.0, 113.4, $113.0(\mathrm{ArC}), 70.0\left(\mathrm{OCH}_{2}\right), 58.1(\mathbf{C H})$, $55.2\left(\mathrm{OCH}_{3}\right) 49.7\left(\mathrm{NHCH}_{2}\right), 35.6\left(\mathrm{ArCH}_{2}\right), 19.6\left(\mathrm{CH}_{3}\right)$.

\section{2-((4-((2-fluorobenzyl)oxy)phenethyl)amino)propanamide (9b)}

Using Method F, to a solution of $\mathbf{4 b}(0.30 \mathrm{~g}, 1.06 \mathrm{mmol}), \mathrm{K}_{2} \mathrm{CO}_{3}(0.58 \mathrm{~g}, 4.26 \mathrm{mmol}), 2-$ Bromopropionamide $(0.24 \mathrm{~g}, 1.60 \mathrm{mmol})$ in $\mathrm{CH}_{3} \mathrm{CN}(10 \mathrm{~mL})$ gave $9 \mathrm{~b}$ as a white solid $(0.12 \mathrm{~g}$, $34 \%) ; R_{f}=0.33\left(\mathrm{CH}_{2} \mathrm{Cl}_{2} 9: \mathrm{MeOH} 1\right) ;{ }^{1} \mathrm{H} \mathrm{NMR}\left(400 \mathrm{MHz}, \mathrm{CDCl}_{3}\right) \delta 7.52-7.48(\mathrm{~m}, 1 \mathrm{ArH})$, 7.35-7.26 (m, 1 ArH), 7.17-6.91 (m, 6 ArH, C(O)NHH'), 5.68 (br s, C(O)NHH'), 5.11 (s, $\left.\mathrm{OCH}_{2}\right), 3.15$ (q, $\left.J=7.0 \mathrm{~Hz}, \mathrm{CH}\right) 2.91-2.69\left(\mathrm{~m}, \mathrm{NHCH}_{\mathbf{2}} \mathrm{CH}_{2}\right), 1.27\left(\mathrm{~d}, J=6.9 \mathrm{~Hz}, \mathrm{CH}_{3}\right) ;{ }^{13} \mathrm{C}$ NMR $\left(75 \mathrm{MHz}, \mathrm{CDCl}_{3}\right) \delta 178.5(\mathbf{C}(\mathrm{O})), 160.5\left(\mathrm{~d}, J_{\mathrm{C}-\mathrm{F}}=245.4 \mathrm{~Hz}\right), 157.1,132.2,129.7,129.6$, 124.3, 124.2, 115.5, 115.2, $115.0(\mathrm{ArC}), 63.8\left(\mathrm{OCH}_{2}\right), 58.1(\mathrm{CH}), 49.8\left(\mathrm{NHCH}_{2}\right), 35.6$ $\left(\mathrm{ArCH}_{2}\right), 19.7\left(\mathrm{CH}_{3}\right)$. 


\section{2-((4-((3-fluorobenzyl)oxy)phenethyl)amino)propanamide (9c)}

Using Method F, to a solution of $4 \mathbf{c}(0.35 \mathrm{~g}, 1.25 \mathrm{mmol}), \mathrm{K}_{2} \mathrm{CO}_{3}(0.69 \mathrm{~g}, 4.98 \mathrm{mmol})$, 2Bromopropionamide $(0.23 \mathrm{~g}, 1.49 \mathrm{mmol})$ in $\mathrm{CH}_{3} \mathrm{CN}(12 \mathrm{~mL})$ gave $9 \mathrm{c}$ as a white solid $(0.09 \mathrm{~g}$, $23 \%) ; R_{f}=0.33\left(\mathrm{CH}_{2} \mathrm{Cl}_{2} 9: \mathrm{MeOH} 1\right) ;{ }^{1} \mathrm{H} \mathrm{NMR}\left(300 \mathrm{MHz}, \mathrm{CDCl}_{3}\right) \delta$ 7.38-7.31 (m, $\left.1 \mathrm{ArH}\right)$, 7.20-7.10 (m, 4 ArH), 7.04-6.89 (m, 3 ArH, C(O)NHH'), 5.33 (br s, C(O)NHH'), 5.04 (s, $\left.\mathrm{OCH}_{2}\right), 3.15$ (q, $\left.J=7.0 \mathrm{~Hz}, \mathrm{CH}\right)$ 2.94-2.69 (m, $\left.\mathrm{NHCH}_{2} \mathrm{CH}_{2}\right), 1.28\left(\mathrm{~d}, J=7.0 \mathrm{~Hz}, \mathrm{CH}_{3}\right) ;{ }^{13} \mathrm{C}$ NMR $\left(75 \mathrm{MHz}, \mathrm{CDCl}_{3}\right) \delta 178.1(\mathbf{C}(\mathrm{O})), 163.0\left(\mathrm{~d}, J_{\mathrm{C}-\mathrm{F}}=244.6 \mathrm{~Hz}\right), 157.1,139.7\left(\mathrm{~d}, J_{\mathrm{C}-\mathrm{F}}=7.3\right.$ $\mathrm{Hz}), 132.2,130.1\left(\mathrm{~d}, J_{\mathrm{C}-\mathrm{F}}=8.2 \mathrm{~Hz}\right), 129.8,122.7\left(\mathrm{~d}, J_{\mathrm{C}-\mathrm{F}}=2.9 \mathrm{~Hz}\right), 114.9,114.7,114.2\left(\mathrm{~d}, J_{\mathrm{C}-\mathrm{F}}\right.$ $=21.9 \mathrm{~Hz})(\mathrm{ArC}), 69.3\left(\mathrm{OCH}_{2}\right), 58.2(\mathbf{C H}), 49.8\left(\mathrm{NHCH}_{2}\right), 35.6\left(\mathrm{ArCH}_{2}\right), 19.7\left(\mathrm{CH}_{3}\right)$.

\section{2-((4-((4-fluorobenzyl)oxy)phenethyl)amino)propanamide (9d)}

Using Method F, to a solution of $4 \mathbf{d}(0.50 \mathrm{~g}, 1.77 \mathrm{mmol}), \mathrm{K}_{2} \mathrm{CO}_{3}(0.98 \mathrm{~g}, 7.10 \mathrm{mmol}), 2-$ Bromopropionamide $(0.32 \mathrm{~g}, 2.13 \mathrm{mmol})$ in $\mathrm{CH}_{3} \mathrm{CN}(17 \mathrm{~mL})$ gave $9 \mathbf{d} \mathbf{k}$ as a white solid $(0.45$ g, 80\%); $R_{f}=0.2\left(\mathrm{CH}_{2} \mathrm{Cl}_{2} 9: \mathrm{MeOH} 1\right) ;{ }^{1} \mathrm{H} \mathrm{NMR}\left(400 \mathrm{MHz}, \mathrm{CDCl}_{3}\right) \delta 7.42-7.38(\mathrm{~m}, 2 \mathrm{ArH})$, 7.12-7.04 (m, 4 ArH), 6.92-6.89 (m, 2 ArH, C(O)NHH'), 5.57 (br s, C(O)NHH'), 5.00 (s, $\left.\mathrm{OCH}_{2}\right), 3.16(\mathrm{q}, J=7.0 \mathrm{~Hz}, \mathrm{CH}) 2.91-2.69\left(\mathrm{~m}, \mathrm{NHCH}_{\mathbf{2}} \mathrm{CH}_{2}\right), 1.27\left(\mathrm{~d}, J=7.0 \mathrm{~Hz}, \mathrm{CH}_{3}\right) ;{ }^{13} \mathrm{C}$ NMR $\left(75 \mathrm{MHz}, \mathrm{CDCl}_{3}\right) \delta 178.5(\mathbf{C}(\mathrm{O})), 162.5\left(\mathrm{~d}, J_{\mathrm{C}-\mathrm{F}}=244.5 \mathrm{~Hz}\right), 157.2,132.8\left(\mathrm{~d}, J_{\mathrm{C}-\mathrm{F}}=3.1\right.$ $\mathrm{Hz}), 132.1,129.7,129.3\left(\mathrm{~d}, J_{\mathrm{C}-\mathrm{F}}=8.2 \mathrm{~Hz}\right), 115.5\left(\mathrm{~d}, J_{\mathrm{C}-\mathrm{F}}=21.5 \mathrm{~Hz}\right), 114.9(\mathrm{ArC}), 63.8\left(\mathrm{OCH}_{2}\right)$, $58.1(\mathbf{C H}), 49.8\left(\mathrm{NHCH}_{2}\right), 35.6\left(\mathrm{ArCH}_{2}\right), 19.7\left(\mathrm{CH}_{3}\right)$.

\section{2-((4-((2-Chlorobenzyl)oxy)phenethyl)amino)propanamide (9e)}

Using Method F, to a solution of $4 \mathrm{e}(0.50 \mathrm{~g}, 1.68 \mathrm{mmol}), \mathrm{K}_{2} \mathrm{CO}_{3}(0.93 \mathrm{~g}, 6.71 \mathrm{mmol})$, 2Bromopropionamide $(0.38 \mathrm{~g}, 2.52 \mathrm{mmol})$ in $\mathrm{CH}_{3} \mathrm{CN}(16 \mathrm{~mL})$ gave $9 \mathrm{e}$ as a white solid $(0.40 \mathrm{~g}$, $71 \%) ; R_{f}=0.33\left(\mathrm{CH}_{2} \mathrm{Cl}_{2} 9: \mathrm{MeOH} 1\right) ;{ }^{1} \mathrm{H} \mathrm{NMR}\left(400 \mathrm{MHz}, \mathrm{CDCl}_{3}\right) \delta$ 7.56-7.54 (m, $\left.1 \mathrm{ArH}\right)$, 7.40-7.38 (m, 1 ArH), 7.30-7.28 (m, 2 ArH), 7.12 (d, J= 8.4 Hz, 2 ArH), 6.94-6.92 (m, 2 ArH, C(O)NHH'), 5.59 (br s, C(O)NHH'), 5.15 (s, OCH $), 3.15$ (q, J= 7.0 Hz, CH) 2.92-2.69 (m, $\left.\mathrm{NHCH}_{2} \mathrm{CH}_{2}\right), 1.27$ (d, $\left.J=7.0 \mathrm{~Hz}, \mathrm{CH}_{3}\right) ;{ }^{13} \mathrm{C} \mathrm{NMR}\left(75 \mathrm{MHz}, \mathrm{CDCl}_{3}\right) \delta 178.4(\mathbf{C}(\mathrm{O})), 157.1$, 134.8, 132.6, 132.2, 129.8, 129.4, 129.0, 128.8, 127.0, $115.0(\mathrm{ArC}), 67.2\left(\mathrm{OCH}_{2}\right), 58.1(\mathbf{C H})$, $49.8\left(\mathrm{NHCH}_{2}\right), 35.6\left(\mathrm{ArCH}_{2}\right), 19.7\left(\mathrm{CH}_{3}\right)$.

\section{2-((4-((3-Chlorobenzyl)oxy)phenethyl)amino)propanamide (9f)}

Using Method F, to a solution of $\mathbf{4 f}(0.50 \mathrm{~g}, 1.68 \mathrm{mmol}), \mathrm{K}_{2} \mathrm{CO}_{3}(0.93 \mathrm{~g}, 6.71 \mathrm{mmol})$, 2Bromopropionamide $(0.38 \mathrm{~g}, 2.52 \mathrm{mmol})$ in $\mathrm{CH}_{3} \mathrm{CN}(16 \mathrm{~mL})$ gave $9 \mathrm{f}$ as a white solid $(0.43 \mathrm{~g}$, $76 \%) ; R_{f}=0.47\left(\mathrm{CH}_{2} \mathrm{Cl}_{2} 9: \mathrm{MeOH} 1\right) ;{ }^{1} \mathrm{H} \mathrm{NMR}\left(400 \mathrm{MHz}, \mathrm{CDCl}_{3}\right) \delta 7.43(\mathrm{~s}, 1 \mathrm{ArH}), 7.33-$ 7.30 (m, 3 ArH), 7.11 (d, $J=8.4$ Hz, 2 ArH), 6.91-6.88 (m, 2 ArH, C(O)NHH'), 5.62 (br s, C(O)NHH'), 5.02 (s, OCH $), 3.15$ (q, $J=6.9 \mathrm{~Hz}, \mathrm{CH})$ 2.91-2.69 (m, NHCH $\left.\mathbf{C H}_{2}\right), 1.27$ (d, $J$ 
$\left.=7.0 \mathrm{~Hz}, \mathrm{CH}_{3}\right) ;{ }^{13} \mathrm{C} \mathrm{NMR}\left(75 \mathrm{MHz}, \mathrm{CDCl}_{3}\right) \delta 178.4(\mathbf{C}(\mathrm{O})), 157.1,139.2,132.3,129.9,129.8$, 128.1, 127.4, 125.4, 125.4, $115.0(\mathrm{ArC}), 69.2\left(\mathrm{OCH}_{2}\right), 58.1(\mathrm{CH}), 49.8\left(\mathrm{NHCH}_{2}\right), 35.6$ $\left(\mathrm{ArCH}_{2}\right), 19.7\left(\mathrm{CH}_{3}\right)$.

\section{2-((4-((4-Chlorobenzyl)oxy)phenethyl)amino)propanamide (9g)}

Using Method F, to a solution of $4 \mathrm{~g}(0.50 \mathrm{~g}, 1.68 \mathrm{mmol}), \mathrm{K}_{2} \mathrm{CO}_{3}(0.93 \mathrm{~g}, 6.71 \mathrm{mmol})$, 2Bromopropionamide $(0.30 \mathrm{~g}, 2.01 \mathrm{mmol})$ in $\mathrm{CH}_{3} \mathrm{CN}(16 \mathrm{~mL})$ gave $9 \mathrm{~g}$ as a white solid $(0.27 \mathrm{~g}$, $48 \%) ; R_{f}=0.33\left(\mathrm{CH}_{2} \mathrm{Cl}_{2} 9: \mathrm{MeOH} 1\right) ;{ }^{1} \mathrm{H} \mathrm{NMR}\left(400 \mathrm{MHz}, \mathrm{CDCl}_{3}\right) \delta$ 7.37-7.33 (m, $\left.4 \mathrm{ArH}\right)$, 7.10 (d, $J=8.5 \mathrm{~Hz}, 2 \mathrm{ArH}), 6.92$ (br s, C(O)NHH'), 6.89 (d, $J=8.5 \mathrm{~Hz}, 2 \mathrm{ArH}), 5.67$ (br s, $\mathrm{C}(\mathrm{O}) \mathrm{NHH}$ ), 5.00 (s, OCH $), 3.15$ (q, $J=7.0 \mathrm{~Hz}, \mathrm{CH})$ 2.91-2.69 (m, NHCH $\left.\mathbf{C H}_{2}\right), 1.27$ (d, $J$ $\left.=6.9 \mathrm{~Hz}, \mathrm{CH}_{3}\right) ;{ }^{13} \mathrm{C}$ NMR $\left(75 \mathrm{MHz}, \mathrm{DMSO}-d_{6}\right) \delta 178.4(\mathbf{C}(\mathrm{O})), 157.1,135.6,133.7,132.2$, 129.7, 128.8, $114.9(\mathrm{ArC}), 69.3\left(\mathrm{OCH}_{2}\right), 58.1(\mathbf{C H}), 49.8\left(\mathrm{NHCH}_{2}\right), 35.6\left(\mathrm{ArCH}_{2}\right), 19.7\left(\mathrm{CH}_{3}\right)$.

\section{2-((4-((2-(trifluoromethyl)benzyl)oxy)phenethyl)amino)propanamide (9h)}

Using Method F, to a solution of $4 \mathbf{h}(0.50 \mathrm{~g}, 1.51 \mathrm{mmol}), \mathrm{K}_{2} \mathrm{CO}_{3}(0.83 \mathrm{~g}, 6.03 \mathrm{mmol})$, 2Bromopropionamide $(0.34 \mathrm{~g}, 2.26 \mathrm{mmol})$ in $\mathrm{CH}_{3} \mathrm{CN}(15 \mathrm{~mL})$ gave $9 \mathbf{h}$ as a white solid $(0.44 \mathrm{~g}$, $80 \%) ; R_{f}=0.37\left(\mathrm{CH}_{2} \mathrm{Cl}_{2} 9: \mathrm{MeOH} 1\right) ;{ }^{1} \mathrm{H} \mathrm{NMR}\left(400 \mathrm{MHz}, \mathrm{CDCl}_{3}\right) \delta 7.74(\mathrm{~d}, J=7.8 \mathrm{~Hz}, 2$ ArH), 7.69 (d, $J=7.8 \mathrm{~Hz}, 2 \mathrm{ArH}), 7.56(\mathrm{t}, J=7.6 \mathrm{~Hz}, 1 \mathrm{ArH}), 7.41$ (t, $J=7.6 \mathrm{~Hz}, 1 \mathrm{ArH}), 7.11$ (d, $J=8.4$ Hz, 2 ArH), 6.92-6.90 (m, 2 ArH, C(O)NHH'), 5.63-5.52 (m, C(O)NHH'), 5.26 (s, $\left.\mathrm{OCH}_{2}\right), 3.15$ (q, $\left.J=6.9 \mathrm{~Hz}, \mathrm{CH}\right) 2.95-2.66\left(\mathrm{~m}, \mathrm{NHCH}_{\mathbf{2}} \mathrm{CH}_{2}\right), 1.27\left(\mathrm{~d}, J=7.0 \mathrm{~Hz}, \mathrm{CH}_{3}\right) ;{ }^{13} \mathrm{C}$ $\operatorname{NMR}\left(75 \mathrm{MHz}, \mathrm{CDCl}_{3}\right) \delta 178.4(\mathbf{C}(\mathrm{O})), 156.9,135.8,132.2,132.1,129.8,128.6,127.7,127.3$ $\left(\mathrm{q}, J_{\mathrm{C}-\mathrm{F}}=30.7 \mathrm{~Hz}\right), 125.9\left(\mathrm{q}, J_{\mathrm{C}-\mathrm{F}}=5.6 \mathrm{~Hz}\right), 124.4\left(\mathrm{q}, J_{\mathrm{C}-\mathrm{F}}=272.1 \mathrm{~Hz}, \mathbf{C F}_{3}\right), 114.9(\mathrm{ArC}), 66.2$ $\left(\mathrm{OCH}_{2}\right), 58.1(\mathbf{C H}), 49.8\left(\mathrm{NHCH}_{2}\right), 35.6\left(\mathrm{ArCH}_{2}\right), 19.6\left(\mathrm{CH}_{3}\right)$.

\section{2-((4-((3-(trifluoromethyl)benzyl)oxy)phenethyl)amino)propanamide (9i)}

Using Method F, to a solution of $4 \mathbf{i}(0.45 \mathrm{~g}, 1.36 \mathrm{mmol}), \mathrm{K}_{2} \mathrm{CO}_{3}(0.75 \mathrm{~g}, 5.44 \mathrm{mmol})$, 2Bromopropionamide $(0.25 \mathrm{~g}, 1.63 \mathrm{mmol})$ in $\mathrm{CH}_{3} \mathrm{CN}(13 \mathrm{~mL})$ gave $9 \mathbf{i}$ as a white solid $(0.23 \mathrm{~g}$, $46 \%) ; R_{f}=0.3\left(\mathrm{CH}_{2} \mathrm{Cl}_{2} 9: \mathrm{MeOH} 1\right) ;{ }^{1} \mathrm{H} \mathrm{NMR}\left(300 \mathrm{MHz}, \mathrm{CDCl}_{3}\right) \delta 7.70-7.48(\mathrm{~m}, 4 \mathrm{ArH})$, 7.13 (d, $J=8.6$ Hz, 2 ArH), 6.93-6.90 (m, 2 ArH, C(O)NHH'), 5.61 (br s, C(O)NHH'), 5.09 $\left(\mathrm{s}, \mathrm{OCH}_{2}\right), 3.16(\mathrm{q}, J=7.0 \mathrm{~Hz}, \mathrm{CH}) 2.94-2.70\left(\mathrm{~m}, \mathrm{NHCH}_{2} \mathbf{C H}_{2}\right), 1.27\left(\mathrm{~d}, J=7.0 \mathrm{~Hz}, \mathrm{CH}_{3}\right) ;{ }^{13} \mathrm{C}$ $\operatorname{NMR}\left(75 \mathrm{MHz}, \mathrm{CDCl}_{3}\right) \delta 178.3(\mathbf{C}(\mathrm{O})), 157.0,138.2,132.4,131.0\left(\mathrm{q}, J_{\mathrm{C}-\mathrm{F}}=32.1 \mathrm{~Hz}\right), 130.6$, $129.8,129.1,124.8\left(\mathrm{q}, J_{\mathrm{C}-\mathrm{F}}=3.7 \mathrm{~Hz}\right), 124.1\left(\mathrm{q}, J_{\mathrm{C}-\mathrm{F}}=3.7 \mathrm{~Hz}\right) 124.0\left(\mathrm{q}, J_{\mathrm{C}-\mathrm{F}}=270.7 \mathrm{~Hz}, \mathbf{C F}_{3}\right)$, $114.9(\mathrm{ArC}) 69.3\left(\mathrm{OCH}_{2}\right), 58.2(\mathbf{C H}), 49.8\left(\mathrm{NHCH}_{2}\right), 35.6\left(\mathrm{ArCH}_{2}\right), 19.7\left(\mathrm{CH}_{3}\right)$.

\section{2-((4-((4-(trifluoromethyl)benzyl)oxy)phenethyl)amino)propanamide (9j)}


Using Method F, to a solution of $\mathbf{4 j}(0.70 \mathrm{~g}, 2.11 \mathrm{mmol}), \mathrm{K}_{2} \mathrm{CO}_{3}(1.17 \mathrm{~g}, 8.46 \mathrm{mmol})$, 2Bromopropionamide $(0.46 \mathrm{~g}, 3.03 \mathrm{mmol})$ in $\mathrm{CH}_{3} \mathrm{CN}(21 \mathrm{~mL})$ gave $9 \mathbf{j}$ as a white solid $(0.75 \mathrm{~g}$, $96 \%) ; R_{f}=0.33\left(\mathrm{CH}_{2} \mathrm{Cl}_{2} 9: \mathrm{MeOH} 1\right) ;{ }^{1} \mathrm{H} \mathrm{NMR}\left(300 \mathrm{MHz}, \mathrm{CDCl}_{3}\right) \delta 7.64(\mathrm{~d}, J=8.3 \mathrm{~Hz}, 2$ $\operatorname{ArH}), 7.55(\mathrm{~d}, J=8.1 \mathrm{~Hz}, 2 \operatorname{ArH}), 7.12(\mathrm{~d}, J=8.6 \mathrm{~Hz}, 2 \operatorname{ArH}), 6.93-6.89$ (m, 2 ArH, C(O)NHH'), 5.44 (br s, C(O)NHH'), 5.11 (s, OCH $), 3.16$ (q, J= $7.0 \mathrm{~Hz}, \mathrm{CH})$ 2.93-2.70 (m, $\left.\mathrm{NHCH}_{2} \mathrm{CH}_{2}\right), 1.28\left(\mathrm{~d}, J=7.0 \mathrm{~Hz}, \mathrm{CH}_{3}\right) ;{ }^{13} \mathrm{C} \mathrm{NMR}\left(75 \mathrm{MHz}, \mathrm{CDCl}_{3}\right) \delta 178.2(\mathbf{C}(\mathrm{O})), 157.0$, $141.2,132.4,130.1\left(\mathrm{q}, J_{\mathrm{C}-\mathrm{F}}=32.6 \mathrm{~Hz}\right), 129.8,127.4,125.5\left(\mathrm{q}, J_{\mathrm{C}-\mathrm{F}}=3.8 \mathrm{~Hz}\right), 124.1\left(\mathrm{q}, J_{\mathrm{C}-\mathrm{F}}=\right.$ $\left.270.3 \mathrm{~Hz}, \mathbf{C F}_{3}\right), 114.9(\mathrm{ArC}) 69.2\left(\mathrm{OCH}_{2}\right), 58.2(\mathbf{C H}), 49.8\left(\mathrm{NHCH}_{2}\right), 35.6\left(\mathrm{ArCH}_{2}\right), 19.7$ $\left(\mathrm{CH}_{3}\right)$.

\section{2-((4-((2,4-Dichlorobenzyl)oxy)phenethyl)amino)propanamide (9k)}

Using Method F, to a solution of $4 \mathbf{k}(0.30 \mathrm{~g}, 0.90 \mathrm{mmol}), \mathrm{K}_{2} \mathrm{CO}_{3}(0.50 \mathrm{~g}, 3.61 \mathrm{mmol})$, 2Bromopropionamide $(0.20 \mathrm{~g}, 1.35 \mathrm{mmol})$ in $\mathrm{CH}_{3} \mathrm{CN}(9 \mathrm{~mL})$ gave $9 \mathrm{k}$ as a white solid $(0.26 \mathrm{~g}$, $79 \%) ; R_{f}=0.30\left(\mathrm{CH}_{2} \mathrm{Cl}_{2} 9: \mathrm{MeOH} 1\right) ;{ }^{1} \mathrm{H} \mathrm{NMR}\left(400 \mathrm{MHz}, \mathrm{CDCl}_{3}\right) \delta 7.49$ (d, $J=8.3 \mathrm{~Hz}, 1$

ArH) 7.42-7.41 (m, 1 ArH), 7.28-7.25 (m, 1 ArH), 7.12 (d, J=8.4 Hz, 2 ArH), 6.91-6.89 (m, $\left.\mathrm{C}(\mathrm{O}) \mathrm{NHH}^{\prime}, 2 \mathrm{ArH}\right), 5.66$ (br s, C(O)NHH'), 5.10 (s, OCH $), 3.16$ (q, $\left.J=7.0 \mathrm{~Hz}, \mathrm{CH}\right) 2.92-$ $2.69\left(\mathrm{~m}, \mathrm{NHCH}_{\mathbf{2}} \mathbf{C H}_{2}\right), 1.27\left(\mathrm{~d}, J=7.0 \mathrm{~Hz}, \mathrm{CH}_{3}\right) ;{ }^{13} \mathrm{C} \mathrm{NMR}\left(75 \mathrm{MHz}, \mathrm{CDCl}_{3}\right) \delta 178.4(\mathbf{C}(\mathrm{O}))$, 156.8, 134.1, 133.5, 133.2, 132.4, 129.8, 129.6, 129.2, 127.3, 114.9 (ArC), $66.7\left(\mathrm{OCH}_{2}\right), 58.1$ $(\mathbf{C H}), 49.7\left(\mathrm{NHCH}_{2}\right), 35.6\left(\mathrm{ArCH}_{2}\right), 19.7\left(\mathrm{CH}_{3}\right)$.

\section{2-((4-((2,4-Dichlorobenzyl)oxy)phenethyl)amino)propanamide (9l)}

Using Method F, to a solution of $41(0.50 \mathrm{~g}, 1.50 \mathrm{mmol}), \mathrm{K}_{2} \mathrm{CO}_{3}(0.83 \mathrm{~g}, 6.01 \mathrm{mmol}), 2-$ Bromopropionamide $(0.34 \mathrm{~g}, 2.25 \mathrm{mmol})$ in $\mathrm{CH}_{3} \mathrm{CN}(15 \mathrm{~mL})$ gave 91 as a white solid $(0.47 \mathrm{~g}$, $85 \%) ; R_{f}=0.38\left(\mathrm{CH}_{2} \mathrm{Cl}_{2} 9: \mathrm{MeOH} 1\right) ;{ }^{1} \mathrm{H} \mathrm{NMR}\left(400 \mathrm{MHz}, \mathrm{CDCl}_{3}\right) \delta 7.53$ (s, $\left.1 \mathrm{ArH}\right), 7.44(\mathrm{~d}$, $J=8.2 \mathrm{~Hz}, 1 \mathrm{ArH}) 7.24$ (s, $1 \mathrm{ArH}), 7.11$ (d, $J=8.4 \mathrm{~Hz}, 2 \mathrm{ArH}), 6.92$ (br s, C(O)NHH'), 6.88 (d, $J=8.5 \mathrm{~Hz}, 2 \mathrm{ArH}), 5.70$ (br s, C(O)NHH'), 4.99 (s, OCH $), 3.15$ (q, $J=7.0 \mathrm{~Hz}, \mathrm{CH}) 2.91$ $2.69\left(\mathrm{~m}, \mathrm{NHCH}_{\mathbf{2}} \mathrm{CH}_{2}\right), 1.27\left(\mathrm{~d}, J=6.9 \mathrm{~Hz}, \mathrm{CH}_{3}\right) ;{ }^{13} \mathrm{C} \mathrm{NMR}\left(75 \mathrm{MHz}, \mathrm{CDCl}_{3}\right) \delta 178.4(\mathbf{C}(\mathrm{O}))$, $156.9,137.4,132.7,132.4,131.9,130.6,129.8,129.2,126.5,114.9(\mathrm{ArC}), 68.6\left(\mathrm{OCH}_{2}\right), 58.1$ $(\mathbf{C H}), 49.7\left(\mathrm{NHCH}_{2}\right), 35.6\left(\mathrm{ArCH}_{2}\right), 19.7\left(\mathrm{CH}_{3}\right)$.

\section{2-methyl-2-((4-((3-fluorobenzyl)oxy)phenethyl)amino)propanamide (9m)}

Using Method F, to a solution of $\mathbf{4 m}(0.35 \mathrm{~g}, 1.25 \mathrm{mmol}), \mathrm{K}_{2} \mathrm{CO}_{3}(0.69 \mathrm{~g}, 4.98 \mathrm{mmol})$, 2Bromo-2-methylpropionamide $(0.25 \mathrm{~g}, 1.49 \mathrm{mmol})$ in $\mathrm{CH}_{3} \mathrm{CN}(12 \mathrm{~mL})$ gave $9 \mathrm{~m}$ as a white solid (0.21 g, 50\%); $R_{f}=0.44\left(\mathrm{CH}_{2} \mathrm{Cl}_{2} 9: \mathrm{MeOH} 1\right) ;{ }^{1} \mathrm{H} \mathrm{NMR}\left(300 \mathrm{MHz}, \mathrm{CDCl}_{3}\right) \delta$ 7.37-6.87 $\left(\mathrm{m}, 8 \mathrm{ArH}, \mathrm{C}(\mathrm{O}) \mathrm{NH}_{2}\right), 5.54$ (br s, NH), $5.04\left(\mathrm{~s}, \mathrm{OCH}_{2}\right), 2.79-2.65\left(\mathrm{~m}, \mathrm{NHCH}_{2} \mathrm{CH}_{2}\right), 1.28$ (s, $\left.\mathrm{CH}_{3} \mathrm{CCH}_{3}\right) ;{ }^{13} \mathrm{C}$ NMR $\left(75 \mathrm{MHz}, \mathrm{CDCl}_{3}\right) \delta 180.0(\mathbf{C}(\mathrm{O})), 163.0\left(\mathrm{~d}, J_{\mathrm{C}-\mathrm{F}}=244.8 \mathrm{~Hz}\right), 157.0$, 
$139.8\left(\mathrm{~d}, J_{\mathrm{C}-\mathrm{F}}=7.3 \mathrm{~Hz}\right), 132.5,130.1\left(\mathrm{~d}, J_{\mathrm{C}-\mathrm{F}}=8.2 \mathrm{~Hz}\right), 129.8,122.7\left(\mathrm{~d}, J_{\mathrm{C}-\mathrm{F}}=2.9 \mathrm{~Hz}\right), 114.9$, $114.8\left(\mathrm{~d}, J_{\mathrm{C}-\mathrm{F}}=20.8 \mathrm{~Hz}\right), 114.2\left(\mathrm{~d}, J_{\mathrm{C}-\mathrm{F}}=21.9 \mathrm{~Hz}\right)(\mathrm{ArC}), 69.3\left(\mathrm{OCH}_{2}\right), 58.8\left(\mathrm{CH}_{3} \mathrm{CCH}_{3}\right), 45.2$ $\left(\mathrm{NHCH}_{2} \mathrm{CH}_{2}\right), 36.3\left(\mathrm{NHCH}_{2} \mathrm{CH}_{2}\right), 25.6\left(\mathrm{CH}_{3} \mathrm{CCH}_{3}\right)$.

\section{2-methyl-2-((4-((3-(trifluoromethyl)benzyl)oxy)phenethyl)amino)propanam ide (9n)}

Using Method F, to a solution of $4 \mathbf{n}(0.45 \mathrm{~g}, 1.36 \mathrm{mmol}), \mathrm{K}_{2} \mathrm{CO}_{3}(0.75 \mathrm{~g}, 5.44 \mathrm{mmol})$, 2-Bromo2-methylpropionamide $(0.27 \mathrm{~g}, 1.63 \mathrm{mmol})$ in $\mathrm{CH}_{3} \mathrm{CN}(13 \mathrm{~mL})$ gave $9 \mathbf{n}$ as a white solid $(0.32$ g, 62\%); $R_{f}=0.3\left(\mathrm{CH}_{2} \mathrm{Cl}_{2} 9: \mathrm{MeOH} 1\right) ;{ }^{1} \mathrm{H} \mathrm{NMR}\left(300 \mathrm{MHz}, \mathrm{CDCl}_{3}\right) \delta 7.70-7.47$ (m, $\left.5 \mathrm{ArH}\right)$, 7.15-6.89 (m, $3 \mathrm{ArH}, \mathrm{C}(\mathrm{O}) \mathrm{NH}_{2}$ ), 5.65 (br s, NH), 5.09 ( $\left.\mathrm{s}, \mathrm{OCH}_{2}\right), 2.79-2.66$ (m, NHCH $\mathbf{C H}_{2}$ ), $1.28\left(\mathrm{~s}, \mathrm{CH}_{3} \mathrm{CCH}_{3}\right) ;{ }^{13} \mathrm{C} \mathrm{NMR}\left(75 \mathrm{MHz}, \mathrm{CDCl}_{3}\right) \delta 180.0(\mathbf{C}(\mathrm{O})), 157.0,138.2,132.7,131.0$ $\left(\mathrm{q}, J_{\mathrm{C}-\mathrm{F}}=32.1 \mathrm{~Hz}\right), 130.6,129.9,129.1,124.8\left(\mathrm{q}, J_{\mathrm{C}-\mathrm{F}}=3.8 \mathrm{~Hz}\right), 124.1\left(\mathrm{q}, J_{\mathrm{C}-\mathrm{F}}=3.9 \mathrm{~Hz}\right), 124.1$ $\left(\mathrm{q}, J_{\mathrm{C}-\mathrm{F}}=270.6 \mathrm{~Hz}, \mathbf{C F}_{3}\right), 114.9(\mathrm{ArC}), 69.3\left(\mathrm{OCH}_{2}\right), 58.8\left(\mathrm{CH}_{3} \mathbf{C C H}_{3}\right), 45.2\left(\mathrm{NHCH}_{2} \mathrm{CH}_{2}\right)$, $36.3\left(\mathrm{NHCH}_{2} \mathrm{CH}_{2}\right), 25.6\left(\mathrm{CH}_{3} \mathrm{CCH}_{3}\right)$.

\section{2-methyl-2-((4-((4-(trifluoromethyl)benzyl)oxy)phenethyl)amino)propanam ide (9o)}

Using Method F, to a solution of $4 \mathbf{o}(0.20 \mathrm{~g}, 0.60 \mathrm{mmol}), \mathrm{K}_{2} \mathrm{CO}_{3}(0.34 \mathrm{~g}, 2.42 \mathrm{mmol}), 2$-Bromo2-methylpropionamide $(0.15 \mathrm{~g}, 0.91 \mathrm{mmol})$ in $\mathrm{CH}_{3} \mathrm{CN}(6 \mathrm{~mL})$ gave 90 as a white solid $(0.32$ g, 62\%); $R_{f}=0.3\left(\mathrm{CH}_{2} \mathrm{Cl}_{2} 9: \mathrm{MeOH} 1\right) ;{ }^{1} \mathrm{H} \mathrm{NMR}\left(300 \mathrm{MHz}, \mathrm{CDCl}_{3}\right) \delta$ 7.66-7.54 (m, $\left.4 \mathrm{ArH}\right)$, 7.14-6.89 (m, $3 \mathrm{ArH}, \mathrm{C}(\mathrm{O}) \mathrm{NH}_{2}$ ), 5.36 (br s, NH), 5.11 ( $\left.\mathrm{s}, \mathrm{OCH}_{2}\right), 2.77-2.68\left(\mathrm{~m}, \mathrm{NHCH}_{2} \mathrm{CH}_{2}\right.$ ), $1.29\left(\mathrm{~s}, \mathrm{CH}_{3} \mathrm{CCH}_{3}\right) ;{ }^{13} \mathrm{C} \mathrm{NMR}\left(75 \mathrm{MHz}, \mathrm{CDCl}_{3}\right) \delta 179.9(\mathbf{C}(\mathrm{O})), 157.0,141.2,132.6,130.1$ $\left(\mathrm{q}, J_{\mathrm{C}-\mathrm{F}}=31.3 \mathrm{~Hz}\right), 129.9,127.4,125.6\left(\mathrm{q}, J_{\mathrm{C}-\mathrm{F}}=3.8 \mathrm{~Hz}\right), 124.8\left(\mathrm{q}, J_{\mathrm{C}-\mathrm{F}}=270.3 \mathrm{~Hz}, \mathbf{C F}_{3}\right)$, $114.8(\mathrm{ArC}), 69.2\left(\mathrm{OCH}_{2}\right), 58.8\left(\mathrm{CH}_{3} \mathrm{CCH}_{3}\right), 45.2\left(\mathrm{NHCH}_{2} \mathrm{CH}_{2}\right), 36.3\left(\mathrm{NHCH}_{2} \mathrm{CH}_{2}\right), 25.6$ $\left(\mathrm{CH}_{3} \mathrm{CCH}_{3}\right)$.

\subsection{Synthesis of 11}

\section{2-((4-(4-(trifluoromethyl)phenoxy)phenethyl)amino)propanamide (11a)}

Using Method F, to a solution of $6 \mathrm{a}(0.30 \mathrm{~g}, 0.94 \mathrm{mmol}), \mathrm{K}_{2} \mathrm{CO}_{3}(0.52 \mathrm{~g}, 3.78 \mathrm{mmol})$, 2Bromopropionamide $(0.17 \mathrm{~g}, 1.13 \mathrm{mmol})$ in $\mathrm{CH}_{3} \mathrm{CN}(9 \mathrm{~mL})$ gave 11a as a white solid $(0.27 \mathrm{~g}$, $79 \%) ; R_{f}=0.26\left(\mathrm{CH}_{2} \mathrm{Cl}_{2} 9.5: \mathrm{MeOH} 0.5\right) ;{ }^{1} \mathrm{H} \mathrm{NMR}\left(300 \mathrm{MHz}, \mathrm{CDCl}_{3}\right) \delta 7.57(\mathrm{~d}, J=8.6 \mathrm{~Hz}$, $2 \mathrm{ArH}), 7.21$ (d, $J=8.4$ Hz, 2 ArH), 7.04-6.93 (m, 4 ArH, C(O)NHH'), 5.73 (br s, C(O)NHH'), 3.19 (q, $J=7.0 \mathrm{~Hz}, \mathrm{CH}), 2.98-2.76\left(\mathrm{~m}, \mathrm{NHCH}_{2} \mathrm{CH}_{2}\right), 1.31$ (d, $J=7.0 \mathrm{~Hz}, \mathrm{CH}_{3}$ ), the NH peak was overlapped with the $\mathrm{CH}_{3}$ signals. The remaining peak was not detected and is believed to overlap with the observed signals; ${ }^{13} \mathrm{C}$ NMR $\left(75 \mathrm{MHz}, \mathrm{CDCl}_{3}\right) \delta 178.2(\mathbf{C}(\mathrm{O})), 160.6,154.2$, $135.9,130.3,127.1\left(\mathrm{q}, J_{\mathrm{C}-\mathrm{F}}=3.7 \mathrm{~Hz}\right), 124.2\left(\mathrm{q}, J_{\mathrm{C}-\mathrm{F}}=269.6 \mathrm{~Hz}\right), 120.0,117.7(\mathrm{ArC}), 58.2$ $(\mathbf{C H}), 49.7\left(\mathrm{NHCH}_{2} \mathrm{CH}_{2}\right), 35.9\left(\mathrm{NHCH}_{2} \mathrm{CH}_{2}\right), 19.7\left(\mathrm{CH}_{3}\right)$. 


\section{2-((4-(3,4-dichlorophenoxy)phenethyl)amino)propanamide (11b)}

Using Method F, to a solution of $\mathbf{6 b}(0.25 \mathrm{~g}, 0.79 \mathrm{mmol}), \mathrm{K}_{2} \mathrm{CO}_{3}(0.43 \mathrm{~g}, 3.14 \mathrm{mmol}), 2-$ Bromopropionamide $(0.14 \mathrm{~g}, 0.94 \mathrm{mmol})$ in $\mathrm{CH}_{3} \mathrm{CN}(8 \mathrm{~mL})$ gave $11 \mathrm{~b}$ as a white solid $(0.25 \mathrm{~g}$, $88 \%) ; R_{f}=0.25\left(\mathrm{CH}_{2} \mathrm{Cl}_{2} 9.5: \mathrm{MeOH} 0.5\right) ;{ }^{1} \mathrm{H} \mathrm{NMR}\left(300 \mathrm{MHz}, \mathrm{CDCl}_{3}\right) \delta 7.37(\mathrm{~d}, J=8.8 \mathrm{~Hz}$, $1 \mathrm{ArH}), 7.20$ (d, $J=8.5 \mathrm{~Hz}, 2 \mathrm{ArH}), 7.06-6.82$ (m, 4 ArH, C(O)NHH'), 5.65 (br s, C(O)NHH'), 3.18 (q, $J=6.9 \mathrm{~Hz}, \mathrm{CH}), 2.97-2.75\left(\mathrm{~m}, \mathrm{NHCH}_{2} \mathrm{CH}_{2}\right), 1.30\left(\mathrm{~d}, J=7.0 \mathrm{~Hz}, \mathrm{CH}_{3}\right)$, the NH peak was overlapped with the $\mathrm{CH}_{3}$ signals. The remaining peak was not detected and is believed to overlap with the observed signals; ${ }^{13} \mathrm{C}$ NMR $\left(75 \mathrm{MHz}, \mathrm{CDCl}_{3}\right) \delta 178.2(\mathbf{C}(\mathrm{O})), 156.8,154.5$, 135.7, 133.2, 131.0, 130.3, 126.3, 120.1, 119.6, 117.8 (ArC), $58.2(\mathrm{CH}), 49.7\left(\mathrm{NHCH}_{2} \mathrm{CH}_{2}\right)$, $35.8\left(\mathrm{NHCH}_{2} \mathbf{C H}_{2}\right), 19.7\left(\mathrm{CH}_{3}\right)$.

\subsection{Synthesis of 13}

\section{2-((2-(4'-(trifluoromethyl)-[1,1'-biphenyl]-4-yl)ethyl)amino)propanamide (13a)}

Using Method F, to a solution of $8 \mathbf{a}(0.30 \mathrm{~g}, 0.99 \mathrm{mmol}), \mathrm{K}_{2} \mathrm{CO}_{3}(0.55 \mathrm{~g}, 3.98 \mathrm{mmol})$, 2bromopropionamide $(0.18 \mathrm{~g}, 1.19 \mathrm{mmol})$ in $\mathrm{CH}_{3} \mathrm{CN}(9 \mathrm{~mL})$ gave 13a as a white solid $(0.21 \mathrm{~g}$, $58 \%) ; R_{f}=0.16\left(\mathrm{CH}_{2} \mathrm{Cl}_{2} 9.5: \mathrm{MeOH} 0.5\right) ;{ }^{1} \mathrm{H} \mathrm{NMR}\left(400 \mathrm{MHz}, \mathrm{CDCl}_{3}\right) \delta 7.68(\mathrm{~s}, 4 \mathrm{ArH}), 7.55$ (d, $J=8.1 \mathrm{~Hz}, 2 \operatorname{ArH}), 7.30$ (d, $J=8.1 \mathrm{~Hz}, 2 \mathrm{ArH}), 6.93$ (br s, C(O)NHH'), 5.58 (br s, C(O)NHH'), 3.20 (q, $J=7.0 \mathrm{~Hz}, \mathrm{CH}), 3.00-2.82$ (m, NHCH $\mathbf{C H}_{2}$ ), 1.45 (br s, NH), 1.30 (d, $J$ $\left.=7.0 \mathrm{~Hz}, \mathrm{CH}_{3}\right) ;{ }^{13} \mathrm{C} \mathrm{NMR}\left(75 \mathrm{MHz}, \mathrm{CDCl}_{3}\right) \delta 178.2(\mathbf{C}(\mathrm{O})), 144.4,139.8,137.8,129.4,129.3$ $\left(\mathrm{q}, J_{\mathrm{C}-\mathrm{F}}=32.1 \mathrm{~Hz}\right), 127.4,127.2,125.7\left(\mathrm{q}, J_{\mathrm{C}-\mathrm{F}}=3.7 \mathrm{~Hz}\right), 124.3\left(\mathrm{q}, J_{\mathrm{C}-\mathrm{F}}=270.2 \mathrm{~Hz}, \mathbf{C F}_{3}\right)$ (ArC), $58.2(\mathbf{C H}), 49.5\left(\mathrm{NHCH}_{2} \mathrm{CH}_{2}\right), 36.2\left(\mathrm{NHCH}_{2} \mathrm{CH}_{2}\right), 19.7\left(\mathrm{CH}_{3}\right)$.

\section{2-((2-(3'-(trifluoromethoxy)-[1,1'-biphenyl]-4-yl)ethyl)amino)propanamide (13b)}

Using Method F, to a solution of $\mathbf{8 b}(0.50 \mathrm{~g}, 1.57 \mathrm{mmol}), \mathrm{K}_{2} \mathrm{CO}_{3}(0.87 \mathrm{~g}, 6.29 \mathrm{mmol})$, 2bromopropionamide $(0.29 \mathrm{~g}, 1.89 \mathrm{mmol})$ in $\mathrm{CH}_{3} \mathrm{CN}(16 \mathrm{~mL})$ gave $\mathbf{1 3 b}$ as a white solid $(0.46$ g, 83\%); $R_{f}=0.08\left(\mathrm{CH}_{2} \mathrm{Cl}_{2} 9.5: \mathrm{MeOH} 0.5\right) ;{ }^{1} \mathrm{H} \mathrm{NMR}\left(400 \mathrm{MHz}, \mathrm{CDCl}_{3}\right) \delta 7.53-7.42(\mathrm{~m}, 5$ ArH), 7.30-7.18 (m, 3 ArH), 6.94 (br s, C(O)NHH'), 5.69 (br s, C(O)NHH'), 3.19 (q, J=7.0 $\mathrm{Hz}, \mathrm{CH}), 2.99-2.81\left(\mathrm{~m}, \mathrm{NHCH}_{2} \mathrm{CH}_{2}\right), 1.30\left(\mathrm{~d}, J=7.0 \mathrm{~Hz}, \mathrm{CH}_{3}\right)$, the NH peak was overlapped with the $\mathrm{CH}_{3}$ signals. The remaining peak was not detected and is believed to overlap with the observed signals; ${ }^{13} \mathrm{C}$ NMR $\left(75 \mathrm{MHz}, \mathrm{CDCl}_{3}\right) \delta 178.2(\mathbf{C}(\mathrm{O})), 149.7,143.0,139.6,137.7,130.1$, 129.3, 127.2, 125.3, $120.6\left(\mathrm{q}, J_{\mathrm{C}-\mathrm{F}}=255.5 \mathrm{~Hz}, \mathbf{C F}_{3}\right), 119.5,119.4(\mathrm{ArC}), 58.2(\mathbf{C H}), 49.5$ $\left(\mathrm{NHCH}_{2} \mathrm{CH}_{2}\right), 36.1\left(\mathrm{NHCH}_{2} \mathbf{C H}_{2}\right), 19.7\left(\mathrm{CH}_{3}\right)$.

\section{2-((2-(4'-(trifluoromethoxy)-[1,1'-biphenyl]-4-yl)ethyl)amino)propanamide (13c)}

Using Method F, to a solution of 8c (0.50 g, $1.57 \mathrm{mmol}), \mathrm{K}_{2} \mathrm{CO}_{3}(0.87 \mathrm{~g}, 6.29 \mathrm{mmol})$, 2bromopropionamide $(0.29 \mathrm{~g}, 1.89 \mathrm{mmol})$ in $\mathrm{CH}_{3} \mathrm{CN}(16 \mathrm{~mL})$ gave $13 \mathrm{c}$ as a white solid $(0.27$ 
g, 49\%); $R_{f}=0.17\left(\mathrm{CH}_{2} \mathrm{Cl}_{2} 9.5: \mathrm{MeOH} 0.5\right) ;{ }^{1} \mathrm{H} \mathrm{NMR}\left(400 \mathrm{MHz}, \mathrm{CDCl}_{3}\right) \delta 7.60-7.49$ (m, 4 ArH), 7.28 (d, $J=8.2 \mathrm{~Hz}, 4 \mathrm{ArH}), 6.93$ (br s, C(O)NHH'), 5.56 (br s, C(O)NHH'), 3.19 (q, $J$ $=7.0 \mathrm{~Hz}, \mathrm{CH}), 2.99-2.80\left(\mathrm{~m}, \mathrm{NHCH}_{2} \mathrm{CH}_{2}\right), 1.30\left(\mathrm{~d}, J=7.0 \mathrm{~Hz}, \mathrm{CH}_{3}\right)$, the NH peak was overlapped with the $\mathrm{CH}_{3}$ signals. The remaining peak was not detected and is believed to overlap with the observed signals; ${ }^{13} \mathrm{C}$ NMR $\left(75 \mathrm{MHz}, \mathrm{CDCl}_{3}\right) \delta 178.2(\mathbf{C}(\mathrm{O})), 148.6,139.6$, 139.2, 137.9, 129.3, 128.3, 127.2, 121.3, 120.6 (q, $\left.J_{\mathrm{C}-\mathrm{F}}=255.6 \mathrm{~Hz}, \mathbf{C F}_{3}\right)(\mathrm{ArC}), 58.2(\mathbf{C H})$, $49.5\left(\mathrm{NHCH}_{2} \mathrm{CH}_{2}\right), 36.1\left(\mathrm{NHCH}_{2} \mathrm{CH}_{2}\right), 19.7\left(\mathrm{CH}_{3}\right)$.

\section{2-((2-(3',4'-dichloro-[1,1'-biphenyl]-4-yl)ethyl)amino)propanamide (13d)}

Using Method F, to a solution of $8 \mathrm{e}(0.30 \mathrm{~g}, 0.99 \mathrm{mmol}), \mathrm{K}_{2} \mathrm{CO}_{3}(0.55 \mathrm{~g}, 3.97 \mathrm{mmol})$, 2bromopropionamide $(0.18 \mathrm{~g}, 1.19 \mathrm{mmol})$ in $\mathrm{CH}_{3} \mathrm{CN}(9 \mathrm{~mL})$ gave $13 \mathbf{d}$ as a white solid $(0.29 \mathrm{~g}$, $82 \%) ; R_{f}=0.18\left(\mathrm{CH}_{2} \mathrm{Cl}_{2} 9.5: \mathrm{MeOH} 0.5\right) ;{ }^{1} \mathrm{H} \mathrm{NMR}\left(400 \mathrm{MHz}, \mathrm{CDCl}_{3}\right) \delta 7.65$ (s, $\left.1 \mathrm{ArH}\right)$, 7.58-7.39 (m, 4 ArH), 7.27 (d, $J=7.8 \mathrm{~Hz}, 4 \mathrm{ArH}), 6.92$ (br s, C(O)NHH'), 5.65 (br s, C(O)NHH'), 3.19 (q, $J=6.9 \mathrm{~Hz}, \mathrm{CH}), 2.99-2.80$ (m, NHCH $\mathbf{C H}_{2}$ ), 1.29 (d, $J=7.0 \mathrm{~Hz}, \mathrm{CH}_{3}$ ), the NH peak was overlapped with the $\mathrm{CH}_{3}$ signals. The remaining peak was not detected and is believed to overlap with the observed signals; ${ }^{13} \mathrm{C} \mathrm{NMR}\left(75 \mathrm{MHz}, \mathrm{CDCl}_{3}\right) \delta 178.2(\mathbf{C}(\mathrm{O}))$, $140.9,139.8,136.8,132.9,131.3,130.7,129.4,128.8,128.5,127.1,126.2(\mathrm{ArC}), 58.2(\mathbf{C H})$, $49.5\left(\mathrm{NHCH}_{2} \mathrm{CH}_{2}\right), 36.2\left(\mathrm{NHCH}_{2} \mathrm{CH}_{2}\right), 19.7\left(\mathrm{CH}_{3}\right)$.

\section{2-((2-(4'-(trifluoromethyl)-[1,1'-biphenyl]-4-yl)ethyl)amino)butanamide (13e)}

Using Method F, to a solution of $8 \mathbf{a}(0.25 \mathrm{~g}, 0.83 \mathrm{mmol}), \mathrm{K}_{2} \mathrm{CO}_{3}(0.23 \mathrm{~g}, 1.66 \mathrm{mmol})$, 2bromobutanamide $(0.14 \mathrm{~g}, 0.83 \mathrm{mmol})$ in $\mathrm{CH}_{3} \mathrm{CN}(10 \mathrm{~mL})$ gave $13 \mathrm{e}$ as a white powder $(0.24$ g, 83\%); $R_{f}=0.48($ EtOAc $9: \mathrm{MeOH} 1) ;{ }^{1} \mathrm{H}$ NMR (400 MHz, DMSO) $\delta 7.87$ (d, $J=8.2 \mathrm{~Hz}, 2$ ArH), 7.79 (d, $J=8.4 \mathrm{~Hz}, 2 \mathrm{ArH}), 7.65(\mathrm{~d}, J=8.2 \mathrm{~Hz}, 2 \operatorname{ArH}), 7.34(\mathrm{~d}, J=8.2 \mathrm{~Hz}, 2 \mathrm{ArH})$, 7.28 (br s, C(O)NHH), 6.96 (br s, C(O)NHH'), 2.89 (t, $J=6.5 \mathrm{~Hz}, \mathrm{CH}), 2.64-2.79$ (m, $\left.\mathrm{NHCH}_{2} \mathrm{CH}_{2}\right), 1.44-1.50\left(\mathrm{~m}, \mathrm{C}(\mathrm{O}) \mathrm{CHCH}_{2}\right), 0.85$ (t, $\left.J=7.4 \mathrm{~Hz}, \mathrm{CH}_{3}\right)$, the NH peak was not detected and is believed to overlap with the observed signals; ${ }^{13} \mathrm{C}$ NMR (100 MHz, DMSO) $\delta$ $177.3(\mathbf{C}(\mathrm{O})), 144.4,141.0,136.7,129.8,129.0\left(\mathrm{~d}, J_{\mathrm{C}-\mathrm{F}}=31.5 \mathrm{~Hz}, \mathbf{C C F}_{3}\right), 127.6,127.5,127.3$, 127.2, 126.2, 126.1, $124.8\left(\mathrm{q}, J_{\mathrm{C}-\mathrm{F}}=270.3 \mathrm{~Hz}, \mathbf{C F}_{3}\right), 63.3(\mathbf{C H}), 49.4\left(\mathrm{NHCH}_{2} \mathrm{CH}_{2}\right), 35.6$ $\left(\mathrm{NHCH}_{2} \mathrm{CH}_{2}\right), 26.4\left(\mathrm{CH}_{2} \mathrm{CH}_{3}\right), 10.6\left(\mathrm{CH}_{3}\right)$.

\section{2-((2-(3',4'-dichloro-[1,1'-biphenyl]-4-yl)ethyl)amino)butanamide (13f)}

Using Method F, to a solution of $8 \mathrm{e}(0.25 \mathrm{~g}, 0.83 \mathrm{mmol}), \mathrm{K}_{2} \mathrm{CO}_{3}(0.23 \mathrm{~g}, 1.66 \mathrm{mmol})$, 2bromobutanamide $(0.14 \mathrm{~g}, 1.20 \mathrm{mmol})$ in $\mathrm{CH}_{3} \mathrm{CN}(10 \mathrm{~mL})$ gave $\mathbf{1 3 f}$ as a white powder $(0.20$ g, 70\%); $R_{f}=0.43$ (EtOAc $\left.9: \mathrm{MeOH} 1\right) ;{ }^{1} \mathrm{H}$ NMR (400 MHz, DMSO) $\delta 7.91(\mathrm{~d}, J=1.9 \mathrm{~Hz}$, ArH), 7.62-7.71 (m, 4 ArH), 7.31 (d, $J=8.2$ Hz, 2 ArH), 7.26 (br s, C(O)NHH), 6.95 (br s, 
$\mathrm{C}(\mathrm{O}) \mathrm{NHH}$ '), 2.87 ( $\mathrm{t}, J=6.5 \mathrm{~Hz}, \mathrm{CH}), 2.62-2.74\left(\mathrm{~m}, \mathrm{NHCH}_{\mathbf{2}} \mathrm{CH}_{2}\right), 1.43-1.49$ (m, C(O)CHCH $)$, $0.84\left(\mathrm{t}, J=7.4 \mathrm{~Hz}, \mathrm{CH}_{3}\right)$, the NH peak was not detected and is believed to overlap with the observed signals; ${ }^{13} \mathrm{C}$ NMR (100 MHz, DMSO) $\delta 177.4(\mathbf{C}(\mathrm{O})), 141.1,141.0,135.6,132.1$, $131.4,130.3,129.8,129.7,128.5,127.1,127.1,127.0,127.0,63.4(\mathrm{CH}), 49.5\left(\mathrm{NHCH}_{2} \mathrm{CH}_{2}\right)$, $35.7\left(\mathrm{NHCH}_{2} \mathrm{CH}_{2}\right), 26.5\left(\mathrm{CH}_{2} \mathrm{CH}_{3}\right), 10.7\left(\mathrm{CH}_{3}\right)$.

\section{2-((2-(4'-(trifluoromethyl)-[1,1'-biphenyl]-4-yl)ethyl)amino)pentanamide (13g)}

Using Method F, to a solution of $\mathbf{8 a}(0.14 \mathrm{~g}, 0.46 \mathrm{mmol}), \mathrm{K}_{2} \mathrm{CO}_{3}(0.13 \mathrm{~g}, 0.92 \mathrm{mmol})$, 2bromopentanamide $(0.09 \mathrm{~g}, 0.51 \mathrm{mmol})$ in $\mathrm{CH}_{3} \mathrm{CN}(8 \mathrm{~mL})$ gave $13 \mathrm{~g}$ as a white powder $(0.10$ g, 61\%); $R_{f}=0.28$ (EtOAc 9 : Hexane 1); ${ }^{1} \mathrm{H}$ NMR (400 MHz, DMSO) $\delta 7.88(\mathrm{~d}, J=8.2 \mathrm{~Hz}$, $2 \operatorname{ArH}), 7.80$ (d, $J=8.3 \mathrm{~Hz}, 2 \mathrm{ArH}), 7.66(\mathrm{~d}, J=8.1 \mathrm{~Hz}, 2 \mathrm{ArH}), 7.34$ (d, $J=8.2 \mathrm{~Hz}, 2 \mathrm{ArH})$, 7.29 (br s, C(O)NHH), 6.95 (br s, C(O)NHH'), 2.96 (t, $J=6.3 \mathrm{~Hz}, \mathrm{CH}), 2.63-2.80$ (m, $\left.\mathrm{NHCH}_{2} \mathrm{CH}_{2}\right), 1.23-1.48\left(\mathrm{~m}, \mathrm{C}(\mathrm{O}) \mathrm{CHCH}_{2} \mathrm{CH}_{2}\right), 0.85$ ( $\left.\mathrm{t}, J=7.2 \mathrm{~Hz}, \mathrm{CH}_{3}\right)$, the NH peak was not detected and is believed to overlap with the observed signals; ${ }^{13} \mathrm{C}$ NMR (100 MHz, DMSO) $\delta 176.9(\mathbf{C}(\mathrm{O})), 144.6,141.4,136.6,129.8,129.8,128.0\left(\mathrm{~d}, J_{\mathrm{C}-\mathrm{F}}=31.8 \mathrm{~Hz}, \mathbf{C C F}_{3}\right), 127.7,127.6$, 127.3, 127.3, 126.2, 126.1, 124.8 (q, $\left.J_{\mathrm{C}-\mathrm{F}}=261.7 \mathrm{~Hz}, \mathrm{CF}_{3}\right), 62.0(\mathbf{C H}), 49.6\left(\mathrm{NHCH}_{2} \mathrm{CH}_{2}\right)$, $36.0\left(\mathrm{NHCH}_{2} \mathbf{C H}_{2}\right), 35.9\left(\mathrm{CH}_{2} \mathrm{CH}_{2} \mathrm{CH}_{3}\right), 19.2\left(\mathrm{CH}_{2} \mathbf{C H}_{2} \mathrm{CH}_{3}\right), 14.4\left(\mathrm{CH}_{3}\right)$.

\section{2-((2-(3',4'-dichloro-[1,1'-biphenyl]-4-yl)ethyl)amino)pentanamide (13h)}

Using Method F, to a solution of $8 \mathrm{e}(0.20 \mathrm{~g}, 0.66 \mathrm{mmol}), \mathrm{K}_{2} \mathrm{CO}_{3}(0.17 \mathrm{~g}, 1.20 \mathrm{mmol})$, 2bromopentanamide $(0.11 \mathrm{~g}, 0.60 \mathrm{mmol})$ in $\mathrm{CH}_{3} \mathrm{CN}(10 \mathrm{~mL})$ gave $\mathbf{1 3 h}$ as a white powder $(0.16$ g, 74\%); $R_{f}=0.32\left(\right.$ EtOAc 9 : Hexane 1); ${ }^{1} \mathrm{H}$ NMR (400 MHz, DMSO) $\delta 7.91(\mathrm{~d}, J=1.8 \mathrm{~Hz}$, ArH), 7.62-7.71 (m, 4 ArH), 7.31 (d, $J=8.1$ Hz, 2 ArH), 7.27 (br s, C(O)NHH), 6.94 (br s, $\mathrm{C}(\mathrm{O}) \mathrm{NHH}$ '), $2.94(\mathrm{t}, J=6.4 \mathrm{~Hz}, \mathrm{CH}), 2.60-2.78\left(\mathrm{~m}, \mathrm{NHCH}_{\mathbf{2}} \mathrm{CH}_{2}\right), 1.24-1.47$ (m, $\left.\mathrm{C}(\mathrm{O}) \mathrm{CHCH}_{2} \mathrm{CH}_{2}\right), 0.85$ ( $\left.\mathrm{t}, J=7.2 \mathrm{~Hz}, \mathrm{CH}_{3}\right)$, the $\mathrm{NH}$ peak was not detected and is believed to overlap with the observed signals; ${ }^{13} \mathrm{C}$ NMR (100 MHz, DMSO) $\delta 177.0(\mathbf{C}(\mathrm{O})), 141.3,141.2$, $135.5,132.1,131.4,130.3,129.8,129.7,128.7,127.1,127.1,127.1,62.0(\mathbf{C H}), 49.6$ $\left(\mathrm{NHCH}_{2} \mathrm{CH}_{2}\right), 36.1\left(\mathrm{NHCH}_{2} \mathbf{C H}_{2}\right), 36.0\left(\mathrm{CH}_{2} \mathrm{CH}_{2} \mathrm{CH}_{3}\right), 19.2\left(\mathrm{CH}_{2} \mathrm{CH}_{3}\right), 14.4\left(\mathrm{CH}_{3}\right)$.

Scheme S4. Synthetic route for $\mathbf{1 5}$ and 16.

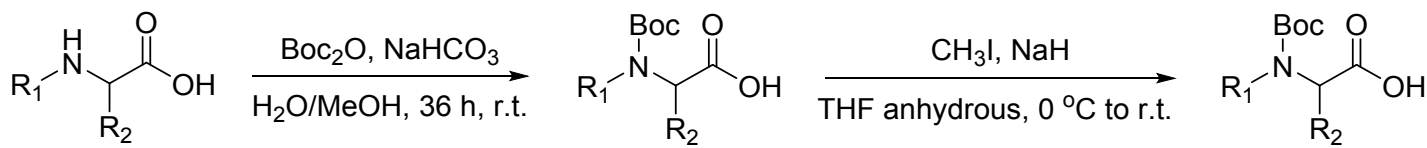

15a; $R_{1}=H, R_{2}=$ ethyl $15 b ; R_{1}=H, R_{2}=$ propyl 15c; $R_{1}=H, R_{2}=n-b u t y l$ 16a; $\mathrm{R}_{1}=\mathrm{CH}_{3}, \mathrm{R}_{2}=$ ethyl 16b; $\mathrm{R}_{1}=\mathrm{CH}_{3}, \mathrm{R}_{2}=$ propyl 16c; $\mathrm{R}_{1}=\mathrm{CH}_{3}, \mathrm{R}_{2}=$ n-butyl

\subsection{Synthesis of 15}

2-((tert-butoxycarbonyl)amino)butanoic acid (15a) 
Using Method $\mathrm{H}$, to a solution of 2-((tert-butoxycarbonyl)amino)butanoic acid (5.00 g, 48.5 mmol), Boc anhydride (19.9 mL, $72.7 \mathrm{mmol}), \mathrm{NaHCO}_{3}(6.11 \mathrm{~g}, 72.7 \mathrm{mmol})$ in distilled water $(125 \mathrm{~mL})$ and methanol $(125 \mathrm{~mL})$ gave 15a as a white solid $(8.25 \mathrm{~g}, 83 \%) ; R_{f}=0.00\left(\mathrm{CH}_{2} \mathrm{Cl}_{2}\right.$ $9.5: \mathrm{MeOH} 0.5$ and few drops of acetic acid); ${ }^{1} \mathrm{H}$ NMR (300 MHz, DMSO- $\left.d_{6}\right) \delta 12.4$ (C(O)OH), 7.02 (d, $J=7.9 \mathrm{~Hz}, \mathrm{NH}), 3.82-3.69$ (m, CH), 1.72-1.48 (m, $\left.\mathrm{CH}_{2} \mathrm{CH}_{3}\right), 1.38$ (s, $\left.\mathrm{C}\left(\mathrm{CH}_{3}\right)_{3}\right), 0.87\left(\mathrm{t}, J=7.3 \mathrm{~Hz}, \mathrm{CH}_{2} \mathrm{CH}_{3}\right) ;{ }^{13} \mathrm{C} \mathrm{NMR}\left(75 \mathrm{MHz}, \mathrm{CDCl}_{3}\right) \delta 174.5(\mathbf{C}(\mathrm{O}) \mathrm{OH}), 156.1$ $\left.(\mathbf{C}(\mathrm{O}) \mathrm{NH}), 78.3\left(\mathbf{C}\left(\mathrm{CH}_{3}\right)_{3}\right), 55.3(\mathbf{C H}), 28.6\left(\mathrm{C}_{(\mathbf{C H}}\right)_{3}\right), 24.6\left(\mathrm{CH}_{2} \mathrm{CH}_{3}\right), 11.0\left(\mathrm{CH}_{2} \mathbf{C H}_{3}\right)$.

\section{2-((tert-butoxycarbonyl)amino)pentanoic acid (15b)}

Using Method $\mathrm{H}$, to a solution of 2-((tert-butoxycarbonyl)amino)pentanoic acid (10.0 g, 85.4 $\mathrm{mmol})$, Boc anhydride $(35.1 \mathrm{~mL}, 128.0 \mathrm{mmol}), \mathrm{NaHCO}_{3}(10.8 \mathrm{~g}, 128.0 \mathrm{mmol})$ in distilled water $(215 \mathrm{~mL})$ and methanol $(215 \mathrm{~mL})$ gave $\mathbf{1 5 b}$ as a white solid $(13.4 \mathrm{~g}, 83 \%) ; R_{f}=0.85$ $\left(\mathrm{CH}_{2} \mathrm{Cl}_{2} 3: \mathrm{MeOH} 17\right) ;{ }^{1} \mathrm{H}$ NMR (400 MHz, DMSO- $\left.d_{6}\right) \delta 12.4(\mathrm{C}(\mathrm{O}) \mathrm{OH}), 7.03(\mathrm{~d}, J=8.0 \mathrm{~Hz}$, $\mathrm{NH}), 3.89-3.75$ (m, $\left.\mathrm{CH}), 1.65-1.50\left(\mathrm{~m}, \mathrm{CH}_{2} \mathrm{CH}_{2} \mathrm{CH}_{3}\right), 1.38-1.20\left(\mathrm{~m}, \mathrm{CH}_{2} \mathrm{CH}_{2} \mathrm{CH}_{3}\right), \mathrm{C}\left(\mathrm{CH}_{3}\right)_{3}\right)$, $0.85\left(\mathrm{t}, J=7.4 \mathrm{~Hz}, \mathrm{CH}_{2} \mathrm{CH}_{2} \mathrm{CH}_{3}\right) ;{ }^{13} \mathrm{C} \mathrm{NMR}\left(100 \mathrm{MHz}, \mathrm{DMSO}-d_{6}\right) \delta 174.8(\mathbf{C}(\mathrm{O}) \mathrm{OH}), 156.1$ $(\mathbf{C}(\mathrm{O}) \mathrm{NH}), \quad 78.4 \quad\left(\mathbf{C}\left(\mathrm{CH}_{3}\right)_{3}\right), \quad 53.6(\mathbf{C H}), \quad 33.3 \quad\left(\mathrm{CH}_{2} \mathrm{CH}_{2} \mathrm{CH}_{3}\right), 28.7 \quad\left(\mathrm{C}_{\left.\left(\mathrm{CH}_{3}\right)_{3}\right),}, 19.3\right.$ $\left(\mathrm{CH}_{2} \mathrm{CH}_{2} \mathrm{CH}_{3}\right), 13.9\left(\mathrm{CH}_{2} \mathrm{CH}_{2} \mathrm{CH}_{3}\right)$.

\section{2-((tert-butoxycarbonyl)amino)hexanoic acid (15c)}

Using Method $\mathrm{H}$, to a solution of 2-((tert-butoxycarbonyl)amino)hexanoic acid (5.50 g, 41.9 mmol), Boc anhydride (17.2 mL, $62.9 \mathrm{mmol}), \mathrm{NaHCO}_{3}(5.28 \mathrm{~g}, 62.9 \mathrm{mmol})$ in distilled water $(105 \mathrm{~mL})$ and methanol $(105 \mathrm{~mL})$ gave $15 \mathrm{c}$ as a white solid $(8.32 \mathrm{~g}, 85 \%) ; R_{f}=0.00\left(\mathrm{CH}_{2} \mathrm{Cl}_{2}\right.$ 3 : MeOH 17); ${ }^{1} \mathrm{H}$ NMR (400 MHz, DMSO- $\left.d_{6}\right) \delta 12.4(\mathrm{C}(\mathrm{O}) \mathrm{OH}), 7.02(\mathrm{~d}, J=8.0 \mathrm{~Hz}, \mathrm{NH})$, 3.87-3.74 (m, $\mathrm{CH}), \quad 1.65-1.54\left(\mathrm{~m}, \quad \mathrm{CH}_{2} \mathrm{CH}_{3}\right), 1.38$ (s, $\left.\mathrm{C}\left(\mathrm{CH}_{3}\right)_{3}\right), \quad 1.35-1.22 \quad(\mathrm{~m}$, $\left.\mathrm{CH}_{2} \mathrm{CH}_{2} \mathrm{CH}_{2} \mathrm{CH}_{3}\right), 0.85$ (t, $\left.J=6.7 \mathrm{~Hz}, \mathrm{CH}_{2} \mathrm{CH}_{2} \mathrm{CH}_{2} \mathrm{CH}_{3}\right) ;{ }^{13} \mathrm{C}$ NMR (75 MHz, DMSO- $\left.d_{6}\right) \delta$ $174.7(\mathbf{C}(\mathrm{O}) \mathrm{OH}), 156.0(\mathbf{C}(\mathrm{O}) \mathrm{NH}), 78.3\left(\mathbf{C}\left(\mathrm{CH}_{3}\right)_{3}\right), 58.8(\mathbf{C H}), 31.0\left(\mathbf{C H}_{2} \mathrm{CH}_{2} \mathrm{CH}_{2} \mathrm{CH}_{3}\right), 28.7$ $\left(\mathrm{C}\left(\mathrm{CH}_{3}\right)_{3}\right), 28.2\left(\mathrm{CH}_{2} \mathrm{CH}_{2} \mathrm{CH}_{2} \mathrm{CH}_{3}\right), 22.1\left(\mathrm{CH}_{2} \mathrm{CH}_{2} \mathrm{CH}_{2} \mathrm{CH}_{3}\right), 14.2\left(\mathrm{CH}_{2} \mathrm{CH}_{2} \mathrm{CH}_{2} \mathbf{C H}_{3}\right)$.

\subsection{Synthesis of 16}

\section{2-((tert-butoxycarbonyl)(methyl)amino)butanoic acid (16a)}

Using Method I, to a solution of $\mathbf{1 5 a}(3.00 \mathrm{~g}, 14.8 \mathrm{mmol})$, Iodomethane $(9.19 \mathrm{~mL}, 147.6 \mathrm{mmol})$ and sodium hydride $(3.54 \mathrm{~g}, 147.6 \mathrm{mmol})$ in THF anhydrous $(147 \mathrm{~mL})$ gave 16a as a yellow oil (2.84 g, 88\%); $R_{f}=0.45\left(\mathrm{CH}_{2} \mathrm{Cl}_{2} 9: \mathrm{MeOH} 1\right.$ and few drops of acetic acid); ${ }^{1} \mathrm{H}$ NMR (300 MHz, DMSO- $\left.d_{6}\right) \delta 12.7(\mathrm{C}(\mathrm{O}) \mathrm{OH}), 4.43-4.14(\mathrm{~m}, \mathrm{CH}), 2.71\left(\mathrm{~s}, \mathrm{NCH}_{3}\right), 1.90-1.36\left(\mathrm{~m}, \mathrm{CH}_{2} \mathrm{CH}_{3}\right.$, $\left.\mathrm{C}\left(\mathrm{CH}_{3}\right)_{3}\right), 0.87-0.79\left(\mathrm{~m}, \mathrm{CH}_{2} \mathrm{CH}_{3}\right) ;{ }^{13} \mathrm{C} \mathrm{NMR}\left(75 \mathrm{MHz}, \mathrm{CDCl}_{3}\right) \delta 173.4(\mathbf{C}(\mathrm{O}) \mathrm{OH}), 156.1$ $(\mathbf{C}(\mathrm{O}) \mathrm{NH}), 79.4\left(\mathbf{C}\left(\mathrm{CH}_{3}\right)_{3}\right), 59.3(\mathbf{C H}), 31.6\left(\mathrm{C}\left(\mathbf{C H}_{3}\right)_{3}\right), 28.5\left(\mathbf{C H}_{2} \mathrm{CH}_{3}\right), 22.5\left(\mathbf{C H}_{3}\right), 11.3$ $\left(\mathrm{CH}_{2} \mathrm{CH}_{3}\right)$. 


\section{2-((tert-butoxycarbonyl)(methyl)amino)pentanoic acid (16b)}

Using Method I, to a solution of $\mathbf{1 5 b}(1.50 \mathrm{~g}, 6.90 \mathrm{mmol})$, Iodomethane $(4.30 \mathrm{~mL}, 69.0 \mathrm{mmol})$ and sodium hydride (1.66 g, $69.0 \mathrm{mmol})$ in THF anhydrous $(70 \mathrm{~mL})$ gave $\mathbf{1 6 b}$ as a yellow oil (1.34 g, 83\%); $R_{f}=0.45\left(\mathrm{CH}_{2} \mathrm{Cl}_{2} 9: \mathrm{MeOH} 1\right.$ and few drops of acetic acid); ${ }^{1} \mathrm{H}$ NMR (300 $\left.\mathrm{MHz}, \mathrm{DMSO}-d_{6}\right) \delta 12.7(\mathrm{C}(\mathrm{O}) \mathrm{OH}), 4.54-4.28(\mathrm{~m}, \mathrm{CH}), 2.70$ (s, NCH $), 1.79-1.64(\mathrm{~m}$, $\mathrm{CH}_{2} \mathrm{CH}_{2} \mathrm{CH}_{3}, 1.41-1.37\left(\mathrm{~m}, \mathrm{CH}_{2} \mathrm{CH}_{2} \mathrm{CH}_{3}, \mathrm{C}\left(\mathrm{CH}_{3}\right)_{3}\right), 1.37-1.29\left(\mathrm{~m}, \mathrm{CH}_{2} \mathrm{CH}_{2} \mathrm{CH}_{3}\right) ;{ }^{13} \mathrm{C} \mathrm{NMR}$ $\left(75 \mathrm{MHz}, \mathrm{CDCl}_{3}\right) \delta 173.6(\mathbf{C}(\mathrm{O}) \mathrm{OH}), 156.0(\mathbf{C}(\mathrm{O}) \mathrm{NH}), 79.4\left(\mathbf{C}\left(\mathrm{CH}_{3}\right)_{3}\right), 58.8(\mathbf{C H}), 31.3\left(\mathbf{C H}_{3}\right)$, $30.7\left(\mathrm{CH}_{2} \mathrm{CH}_{2} \mathrm{CH}_{3}\right), 28.5\left(\mathrm{C}\left(\mathrm{CH}_{3}\right)_{3}\right), 19.4\left(\mathrm{CH}_{2} \mathbf{C H}_{2} \mathrm{CH}_{3}\right), 13.8\left(\mathrm{CH}_{2} \mathrm{CH}_{2} \mathbf{C H}_{3}\right)$.

\section{2-((tert-butoxycarbonyl)(methyl)amino)hexanoic acid (16c)}

Using Method I, 15c (0.50 g, $2.16 \mathrm{mmol})$, iodomethane $(1.35 \mathrm{~mL}, 21.6 \mathrm{mmol})$ and sodium hydride (0.87 g, $21.6 \mathrm{mmol})$ gave 16c as a oil $(0.42 \mathrm{~g}, 78 \%) ; R_{f}=0.33\left(\mathrm{CH}_{2} \mathrm{Cl}_{2} 9: \mathrm{MeOH} 1\right)$; ${ }^{1} \mathrm{H}$ NMR (400 MHz, DMSO- $d_{6}$ ) $\delta 12.6$ (br s, C(O)OH), 4.25-4.52 (m, chiral-H), 2.70 (s, $\left.\mathrm{NCH}_{3}\right), 1.66-1.81\left(\mathrm{~m}, \mathrm{CH}_{2} \mathrm{CH}_{2} \mathrm{CH}_{2} \mathrm{CH}_{3}\right), 1.38$ (d, $\left.J=15.1 \mathrm{~Hz}, \mathrm{C}\left(\mathrm{CH}_{3}\right)_{3}\right), 1.18-1.33$ (m, $\mathrm{CH}_{2} \mathrm{CH}_{2} \mathrm{CH}_{2} \mathrm{CH}_{3}$ ), 0.87 (q, $\mathrm{J}=\mathrm{Hz}, \mathrm{CH}_{2} \mathrm{CH}_{2} \mathrm{CH}_{2} \mathrm{CH}_{3}$ ).

\subsection{Synthesis of 17}

\section{tert-butyl(1-oxo-1-((4-((4-(trifluoromethyl)benzyl)oxy)phenethyl)amino)pentan-2- yl)carbamate (17a)}

Using Method J, to a solution of $\mathbf{3 j}(0.50 \mathrm{~g}, 2.30 \mathrm{mmol})$, NMM (0.51 mL. $4.60 \mathrm{mmol})$, IBCF $(0.39 \mathrm{~mL}, 3.00 \mathrm{mmol}), \mathbf{1 5 b}(0.80 \mathrm{~g}, 2.42 \mathrm{mmol})$ in THF anhydrous $(23 \mathrm{~mL})$ gave $\mathbf{2 8 c}$ as a white solid (1.03 g, 90\%); $R_{f}=0.16$ (EtOAc $1:$ n-hexane 3); ${ }^{1} \mathrm{H}$ NMR $\left(300 \mathrm{MHz}, \mathrm{CDCl}_{3}\right) \delta 7.59$ (dd, $J=21.2,8.2 \mathrm{~Hz}, 4 \mathrm{ArH}), 7.11$ (d, $J=8.6 \mathrm{~Hz}, 2 \mathrm{ArH}), 6.90$ (d, $J=8.6 \mathrm{~Hz}, 2 \mathrm{ArH}), 6.20$ (br s, $\mathrm{C}(\mathrm{O}) \mathrm{NH}), 5.10$ (s, OCH $), 5.03$ (br s, C(O)ONH), 4.00 (q, $J=6.8 \mathrm{~Hz}, \mathrm{CH}), 3.58-3.40$ (m, $\left.\mathrm{NHCH}_{2} \mathrm{CH}_{2}\right), 2.75\left(\mathrm{t}, J=7.1 \mathrm{~Hz}, \mathrm{NHCH}_{2} \mathrm{CH}_{2}\right), 1.81-1.48\left(\mathrm{~m}, \mathrm{CH}_{2} \mathrm{CH}_{2} \mathrm{CH}_{3}\right), 1.43\left(\mathrm{~s}, \mathrm{C}\left(\mathrm{CH}_{3}\right)_{3}\right)$, 1.37-1.25 (m, $\left.\mathrm{CH}_{2} \mathrm{CH}_{2} \mathrm{CH}_{3}\right), 0.90\left(\mathrm{t}, J=7.2 \mathrm{~Hz}, \mathrm{CH}_{2} \mathrm{CH}_{2} \mathrm{CH}_{3}\right.$ ); ${ }^{13} \mathrm{C}$ NMR (75 MHz, DMSO$\left.d_{6}\right) \delta 172.1(\mathbf{C}(\mathrm{O}) \mathrm{NH}), 157.2(\mathrm{ArC}), 155.7(\mathbf{C}(\mathrm{O}) \mathrm{ONH}), 141.2,131.5,130.1\left(\mathrm{q}, J_{\mathrm{C}-\mathrm{F}}=32.2\right.$ $\mathrm{Hz}), 129.8,127.3,125.5\left(\mathrm{q}, J_{\mathrm{C}-\mathrm{F}}=3.7 \mathrm{~Hz}\right), 124.1$ (q, $\left.J_{\mathrm{C}-\mathrm{F}}=270.4 \mathrm{~Hz}, \mathbf{C F}_{3}\right), 115.0(\mathrm{ArC}), 79.9$ $\left(\mathbf{C}\left(\mathrm{CH}_{3}\right)_{3}\right), \quad 69.2 \quad\left(\mathrm{OCH}_{2}\right), \quad 54.5(\mathbf{C H}), 40.8 \quad\left(\mathrm{NHCH}_{2} \mathrm{CH}_{2}\right), 34.9 \quad\left(\mathrm{NHCH}_{2} \mathbf{C H}_{2}\right), \quad 34.8$ $\left(\mathrm{CH}_{2} \mathrm{CH}_{2} \mathrm{CH}_{3}\right), 28.3\left(\mathrm{C}\left(\mathrm{CH}_{3}\right)_{3}\right), 18.9\left(\mathrm{CH}_{2} \mathrm{CH}_{2} \mathrm{CH}_{3}\right), 13.8\left(\mathrm{CH}_{2} \mathrm{CH}_{2} \mathbf{C H}_{3}\right)$.

tert-butyl(1-oxo-1-((4-((3,4-dichlorobenzyl)oxy)phenethyl)amino)pentan-2-yl)carbamate (17b)

Using Method J, to a solution of 31 ( $0.50 \mathrm{~g}, 2.30 \mathrm{mmol})$, NMM (0.51 mL. $4.60 \mathrm{mmol})$, IBCF $(0.39 \mathrm{~mL}, 3.00 \mathrm{mmol}), \mathbf{1 5 b}(0.80 \mathrm{~g}, 2.42 \mathrm{mmol})$ in THF anhydrous $(23 \mathrm{~mL})$ gave $\mathbf{2 8 d}$ as a 
white solid (1.05 g, 92\%); $R_{f}=0.14$ (EtOAc $1:$ n-hexane 3$) ;{ }^{1} \mathrm{H}$ NMR (300 MHz, $\left.\mathrm{CDCl}_{3}\right) \delta$ 7.53-7.52 (m, 1 ArH), 7.44 (d, $J=8.2 \mathrm{~Hz}, 1 \mathrm{ArH}), 7.24-7.23$ (m, 1 ArH), 7.11 (d, $J=8.5 \mathrm{~Hz}$, $2 \operatorname{ArH}), 6.88$ (d, $J=8.6 \mathrm{~Hz}, 2 \mathrm{ArH}), 6.18$ (br s, C(O)NH), 4.98 (br s, OCH, $\mathrm{C}(\mathrm{O}) \mathrm{ONH}), 4.00$ (q, $J=6.6 \mathrm{~Hz}, \mathrm{CH}), 3.58-3.40\left(\mathrm{~m}, \mathrm{NHCH}_{2} \mathrm{CH}_{2}\right), 2.75$ (t, $\left.J=7.1 \mathrm{~Hz}, \mathrm{NHCH}_{2} \mathrm{CH}_{2}\right), 1.78-1.48$ $\left(\mathrm{m}, \mathrm{CH}_{2} \mathrm{CH}_{2} \mathrm{CH}_{3}\right), 1.43\left(\mathrm{~s}, \mathrm{C}\left(\mathrm{CH}_{3}\right)_{3}\right), 1.37-1.26\left(\mathrm{~m}, \mathrm{CH}_{2} \mathrm{CH}_{2} \mathrm{CH}_{3}\right), 0.90$ (t, $J=7.2 \mathrm{~Hz}$, $\left.\mathrm{CH}_{2} \mathrm{CH}_{2} \mathrm{CH}_{3}\right) ;{ }^{13} \mathrm{C}$ NMR $\left(75 \mathrm{MHz}, \mathrm{DMSO}-d_{6}\right) \delta 172.1(\mathbf{C}(\mathrm{O}) \mathrm{NH}), 157.0(\mathrm{ArC}), 155.7$ (C(O)ONH), 137.4, 132.8, 131.9, 131.6, 130.6, 129.8, 129.2, 126.5, 115.0 (ArC), 80.0 $\left(\mathrm{C}_{\left.\left(\mathrm{CH}_{3}\right)_{3}\right),} 68.6\left(\mathrm{OCH}_{2}\right), 54.5(\mathbf{C H}), 40.8\left(\mathrm{NHCH}_{2} \mathrm{CH}_{2}\right), 34.8 \quad\left(\mathrm{NHCH}_{2} \mathrm{CH}_{2}\right), 34.7\right.$ $\left(\mathrm{CH}_{2} \mathrm{CH}_{2} \mathrm{CH}_{3}\right), 28.3\left(\mathrm{C}\left(\mathrm{CH}_{3}\right)_{3}\right), 18.9\left(\mathrm{CH}_{2} \mathrm{CH}_{2} \mathrm{CH}_{3}\right), 13.8\left(\mathrm{CH}_{2} \mathrm{CH}_{2} \mathrm{CH}_{3}\right)$.

tert-butylmethyl(1-oxo-1-((4-((4-(trifluoromethyl)benzyl)oxy)phenethyl)amino)pentan-2yl)carbamate (17c)

Using Method J, to a solution of $\mathbf{3 j}(0.18 \mathrm{~g}, 0.78 \mathrm{mmol})$, NMM (0.17 mL. $1.56 \mathrm{mmol})$, IBCF $(0.13 \mathrm{~mL}, 1.01 \mathrm{mmol}), \mathbf{1 6 b}(0.27 \mathrm{~g}, 0.82 \mathrm{mmol})$ in THF anhydrous $(7 \mathrm{~mL})$ gave $17 \mathbf{c}$ as a colorless oil $(0.33 \mathrm{~g}, 82 \%) ; R_{f}=0.12$ (EtOAc $1: \mathrm{n}$-hexane 3$) ;{ }^{1} \mathrm{H}$ NMR $\left(300 \mathrm{MHz}, \mathrm{CDCl}_{3}\right) \delta$ 7.59 (dd, $J=22.0,8.2 \mathrm{~Hz}, 4$ ArH), 7.12-7.08 (m, 2 ArH), 6.90 (d, $J=8.6$ Hz, 2 ArH), 6.14$5.89(\mathrm{~m}, \mathrm{C}(\mathrm{O}) \mathrm{NH}), 5.10$ (s, OCH $\mathbf{H}_{2}, 4.50$ (br s, CH), 3.57-3.46 (m, $\left.\mathrm{NHCH}_{2} \mathrm{CH}_{2}\right), 2.76-2.72(\mathrm{~m}$, $\left.\mathrm{NHCH}_{2} \mathrm{CH}_{2}\right), 2.65$ (s, $\left.\mathrm{CH}_{3}\right), 1.90-1.54$ (m, $\left.\mathrm{CH}_{2} \mathrm{CH}_{2} \mathrm{CH}_{3}\right), 1.44$ (s, $\left.\mathrm{C}\left(\mathrm{CH}_{3}\right)_{3}\right), 1.26-1.23$ (m, $\mathrm{CH}_{2} \mathrm{CH}_{2} \mathrm{CH}_{3}$ ), 0.93 (t, $\left.J=7.3 \mathrm{~Hz}, \mathrm{CH}_{2} \mathrm{CH}_{2} \mathrm{CH}_{3}\right) ;{ }^{13} \mathrm{C}$ NMR $\left(75 \mathrm{MHz}, \mathrm{DMSO}-d_{6}\right) \delta 171.1$ $(\mathbf{C}(\mathrm{O}) \mathrm{NH}), 157.2,141.2,130.1\left(\mathrm{q}, J_{\mathrm{C}-\mathrm{F}}=32.3 \mathrm{~Hz}\right), 129.8,127.3,125.5\left(\mathrm{q}, J_{\mathrm{C}-\mathrm{F}}=3.8 \mathrm{~Hz}\right), 124.1$ $\left(\mathrm{q}, J_{\mathrm{C}-\mathrm{F}}=270.4 \mathrm{~Hz}, \mathbf{C F}_{3}\right), 115.0(\mathrm{ArC}), 80.4\left(\mathbf{C}\left(\mathrm{CH}_{3}\right)_{3}\right), 69.2\left(\mathrm{OCH}_{2}\right), 57.9(\mathbf{C H}), 40.6$ $\left(\mathrm{NHCH}_{2} \mathrm{CH}_{2}\right), 34.9\left(\mathrm{NHCH}_{2} \mathrm{CH}_{2}\right), 29.8\left(\mathrm{CH}_{2} \mathrm{CH}_{2} \mathrm{CH}_{3}\right), 29.7\left(\mathrm{CH}_{3}\right), 28.4\left(\mathrm{C}_{\left.\left(\mathrm{CH}_{3}\right)_{3}\right), 19.2}\right.$ $\left(\mathrm{CH}_{2} \mathrm{CH}_{2} \mathrm{CH}_{3}\right), 13.7\left(\mathrm{CH}_{2} \mathrm{CH}_{2} \mathbf{C H}_{3}\right)$.

\section{tert-butylmethyl(1-oxo-1-((4-((3,4-dichlorobenzyl)oxy)phenethyl)amino)pentan-2- yl)carbamate (17d)}

Using Method J, to a solution of 31 ( $0.18 \mathrm{~g}, 0.78 \mathrm{mmol})$, NMM (0.17 mL. $1.56 \mathrm{mmol})$, IBCF $(0.13 \mathrm{~mL}, 1.01 \mathrm{mmol}), \mathbf{1 6 b}(0.27 \mathrm{~g}, 0.82 \mathrm{mmol})$ in THF anhydrous $(7 \mathrm{~mL})$ gave $\mathbf{1 7 d}$ as a white solid (0.36 g, 90\%); $R_{f}=0.14$ (EtOAc $1:$ n-hexane 3); ${ }^{1} \mathrm{H}$ NMR $\left(300 \mathrm{MHz}, \mathrm{CDCl}_{3}\right) \delta 7.53$ $7.52(\mathrm{~m}, 1 \mathrm{ArH}), 7.45$ (d, $J=8.2 \mathrm{~Hz}, 1 \mathrm{ArH}), 7.27-7.23$ (m, $1 \mathrm{ArH}), 7.10$ (dd, $J=58.1,8.6 \mathrm{~Hz}$, $4 \mathrm{ArH}), 6.14-5.89$ (m, C(O)NH), 4.98 ( $\left.\mathrm{s}, \mathrm{OCH}_{2}\right), 4.50$ (br s, CH), 3.54-3.46 (m, $\mathrm{NHCH}_{2} \mathrm{CH}_{2}$ ), $2.74\left(\mathrm{t}, J=6.8 \mathrm{~Hz}, \mathrm{NHCH}_{2} \mathrm{CH}_{2}\right), 2.65\left(\mathrm{~s}, \mathrm{CH}_{3}\right), 1.76-1.54\left(\mathrm{~m}, \mathrm{CH}_{2} \mathrm{CH}_{2} \mathrm{CH}_{3}\right), 1.44\left(\mathrm{~s}, \mathrm{C}\left(\mathrm{CH}_{3}\right)_{3}\right)$, 1.27-1.21 (m, $\mathrm{CH}_{2} \mathrm{CH}_{2} \mathrm{CH}_{3}$ ), 0.90 (t, $J=7.3 \mathrm{~Hz}, \mathrm{CH}_{2} \mathrm{CH}_{2} \mathrm{CH}_{3}$ ); ${ }^{13} \mathrm{C}$ NMR (75 MHz, DMSO$\left.d_{6}\right) \delta 171.1(\mathbf{C}(\mathrm{O}) \mathrm{NH}), 157.0,141.2,137.4,132.8,131.6,130.6,129.8,129.2,126.5,115.0$ $(\mathrm{ArC}), 80.4\left(\mathbf{C}\left(\mathrm{CH}_{3}\right)_{3}\right), 68.6\left(\mathrm{OCH}_{2}\right), 57.9(\mathbf{C H}), 40.6\left(\mathrm{NHCH}_{2} \mathrm{CH}_{2}\right), 34.9\left(\mathrm{NHCH}_{2} \mathrm{CH}_{2}\right), 29.8$ $\left(\mathrm{CH}_{2} \mathrm{CH}_{2} \mathrm{CH}_{3}\right), 29.7\left(\mathbf{C H}_{3}\right), 28.4\left(\mathrm{C}\left(\mathrm{CH}_{3}\right)_{3}\right), 19.2\left(\mathrm{CH}_{2} \mathbf{C H}_{2} \mathrm{CH}_{3}\right), 13.7\left(\mathrm{CH}_{2} \mathrm{CH}_{2} \mathbf{C H}_{3}\right)$. 


\subsection{Synthesis of 19}

tert-butyl(1-oxo-1-((4-(4-(trifluoromethyl)phenoxy)phenethyl)amino)pentan-2yl)carbamate (19a)

Using Method J, 5a (0.06 g, $0.27 \mathrm{mmol}), N$-methylmorpholine $(0.07 \mathrm{~mL}, 0.67 \mathrm{mmol})$, isobutyl chloroformate $(0.05 \mathrm{~mL}, 0.35 \mathrm{mmol})$ and $15 \mathbf{b}(0.09 \mathrm{~g}, 0.28 \mathrm{mmol})$ gave 19a as a white solid $(0.13 \mathrm{~g}, 100 \%): R_{f}=0.08$ ( $n$-hexane $3:$ EtOAc 1$) ;{ }^{1} \mathrm{H} \mathrm{NMR}\left(300 \mathrm{MHz}, \mathrm{CDCl}_{3}\right) \delta 7.57(\mathrm{~d}, J=$ 7.0 Hz, 2 ArH), 7.20-7.22 (m, 2 ArH), 6.98-7.04 (m, 4 ArH), 6.13 (br s, C(O)NH), 4.93 (br s, $\mathrm{C}(\mathrm{O}) \mathrm{ONH}), 4.00\left(\mathrm{~d}, J=6.2 \mathrm{~Hz}\right.$, chiral-H), 3.48-3.57 (m, $\left.\mathrm{NHCH}_{2} \mathrm{CH}_{2}\right), 2.82(\mathrm{t}, J=5.8 \mathrm{~Hz}$, $\left.\mathrm{NHCH}_{2} \mathrm{CH}_{2}\right), 1.51-1.79\left(\mathrm{~m}, \mathrm{CH}_{2} \mathrm{CH}_{2} \mathrm{CH}_{3}\right), 1.43$ (d, $\left.J=2.1 \mathrm{~Hz}, \mathrm{C}\left(\mathrm{CH}_{3}\right)_{3}\right), 1.32-1.36$ (m, $\left.\mathrm{CH}_{2} \mathrm{CH}_{2} \mathrm{CH}_{3}\right), 0.90-0.94\left(\mathrm{~m}, \mathrm{CH}_{2} \mathrm{CH}_{2} \mathrm{CH}_{3}\right)$.

\section{tert-butyl(1-((4-(3,4-dichlorophenoxy)phenethyl)amino)-1-oxopentan-2-yl)carbamate} (19b)

Using Method J, 5b (0.14 g, $0.62 \mathrm{mmol}), N$-methylmorpholine $(0.17 \mathrm{~mL}, 1.56 \mathrm{mmol})$, isobutyl chloroformate $(0.11 \mathrm{~mL}, 0.81 \mathrm{mmol})$ and $15 \mathbf{b}(0.19 \mathrm{~g}, 0.60 \mathrm{mmol})$ gave $19 \mathrm{~b}$ as a white solid $(0.21 \mathrm{~g}, 74 \%): R_{f}=0.14$ ( $n$-hexane $3:$ EtOAc 1$) ;{ }^{1} \mathrm{H} \mathrm{NMR}\left(300 \mathrm{MHz}, \mathrm{CDCl}_{3}\right) \delta 7.37(\mathrm{~d}, J=$ $8.8 \mathrm{~Hz}, 1 \mathrm{ArH}), 7.19(\mathrm{~d}, J=8.5 \mathrm{~Hz}, 2 \operatorname{ArH}), 7.06(\mathrm{~d}, J=2.8 \mathrm{~Hz}, 1 \operatorname{ArH}), 6.93-6.96(\mathrm{~m}, 2 \mathrm{ArH})$, 6.82-6.86 (m, 1 ArH), 6.15 (d, J=5.3 Hz, C(O)NH), 4.95 (br s, C(O)ONH), 3.96-4.03 (m, chiral-H), 3.44-3.62 (m, $\left.\mathrm{NHCH}_{2} \mathrm{CH}_{2}\right), 2.81$ (t, $\left.J=9.4 \mathrm{~Hz}, \mathrm{NHCH}_{2} \mathrm{CH}_{2}\right), 1.48-1.81$ (m, $\left.\mathrm{CH}_{2} \mathrm{CH}_{2} \mathrm{CH}_{3}\right), 1.43\left(\mathrm{~s}, \mathrm{C}\left(\mathrm{CH}_{3}\right)_{3}\right), 1.24-1.36\left(\mathrm{~m}, \mathrm{CH}_{2} \mathrm{CH}_{2} \mathrm{CH}_{3}\right), 0.91(\mathrm{t}, J=7.3 \mathrm{~Hz}$, $\mathrm{CH}_{2} \mathrm{CH}_{2} \mathrm{CH}_{3}$ ).

\subsection{Synthesis of 21}

tert-butyl(1-oxo-1-((2-(4'-(trifluoromethyl)-[1,1'-biphenyl]-4-yl)ethyl)amino)butan-2yl)carbamate (21a)

Using Method J, 8a (0.42 g, $2.08 \mathrm{~m} \mathrm{~mol}), N$-methylmorpholine $(0.57 \mathrm{~mL}, 5.21 \mathrm{mmol})$, isobutyl chloroformate $(0.35 \mathrm{~m} \mathrm{~L}, 2.71 \mathrm{mmol})$ and $15 \mathrm{a}(0.66 \mathrm{~g}, 2.19 \mathrm{mmol})$ gave $21 \mathrm{a}$ as a white solid (0.92 g, 98\%): $R_{f}=0.16$ ( $n$-hexane $3:$ EtOAc 1); ${ }^{1} \mathrm{H}$ NMR $\left(400 \mathrm{MHz}, \mathrm{CDCl}_{3}\right) \delta 7.69-7.71(\mathrm{~m}$, 4 ArH), 7.57 (d, $J=8.0 \mathrm{~Hz}, 2 \operatorname{ArH}), 7.32$ (d, $J=8.0 \mathrm{~Hz}, 2 \mathrm{ArH}), 6.15$ (br s, C(O)NH), 5.00 (br s, C(O)ONH), 3.97 (q, $J=6.8 \mathrm{~Hz}, 8.4 \mathrm{~Hz}$, chiral-H), 3.53-3.66 (s, $\left.\mathrm{NHCH}_{2} \mathrm{CH}_{2}\right), 2.90$ (d, $J$ $\left.=7.0 \mathrm{~Hz}, \mathrm{NHCH}_{2} \mathrm{CH}_{2}\right), 1.58-1.90\left(\mathrm{~m}, \mathrm{CH}_{2} \mathrm{CH}_{3}\right), 1.45\left(\mathrm{~s}, \mathrm{C}\left(\mathrm{CH}_{3}\right)_{3}\right), 0.93(\mathrm{~d}, J=7.4 \mathrm{~Hz}$, $\mathrm{CH}_{2} \mathrm{CH}_{3}$ ).

\section{tert-butyl(1-ox0-1-((2-(4'-(trifluoromethoxy)-[1,1'-biphenyl]-4-yl)ethyl)amino)butan-2-} yl)carbamate (21b)

Using Method J, 8c (0.49 g, $2.40 \mathrm{~m} \mathrm{~mol}), N$-methylmorpholine $(0.66 \mathrm{~mL}, 6.00 \mathrm{mmol})$, isobutyl chloroformate $(0.41 \mathrm{~m} \mathrm{~L}, 3.12 \mathrm{mmol})$ and $\mathbf{1 5 a}(0.80 \mathrm{~g}, 2.52 \mathrm{mmol})$ gave $\mathbf{2 1 b}$ as a white solid $(0.94 \mathrm{~g}, 84 \%): R_{f}=0.14$ ( $n$-hexane $3:$ EtOAc 1$) ;{ }^{1} \mathrm{H}$ NMR $\left(400 \mathrm{MHz}, \mathrm{CDCl}_{3}\right) \delta 7.68-7.73(\mathrm{~m}$, 3 ArH), 7.51-7.61 (m, 2 ArH), 7.31 (d, $J=8.2 \mathrm{~Hz}, 2 \mathrm{ArH}), 6.17$ (d, $J=5.6 \mathrm{~Hz}, \mathrm{C}(\mathrm{O}) \mathrm{NH}), 5.01$ 
(br s, C(O)ONH), 3.96 (t, $J=6.7 \mathrm{~Hz}$, chiral-H), 3.51-3.67 (m, $\left.\mathrm{NHCH}_{2} \mathrm{CH}_{2}\right), 2.90(\mathrm{~d}, J=7.0$ $\left.\mathrm{Hz}, \mathrm{NHCH}_{2} \mathrm{CH}_{2}\right), 1.56-1.92\left(\mathrm{~m}, \mathrm{CH}_{2} \mathrm{CH}_{3}\right), 1.45$ (s, $\left.\mathrm{C}\left(\mathrm{CH}_{3}\right)_{3}\right), 0.93$ (d, J=7.4 Hz, $\left.\mathrm{CH}_{2} \mathrm{CH}_{3}\right)$.

tert-butyl(1-((2-(3',4'-difluoro-[1,1'-biphenyl]-4-yl)ethyl)amino)-1-oxobutan-2yl)carbamate (21c)

Using Method J, 8d (0.65 g, $3.18 \mathrm{mmol}), N$-methylmorpholine (0.98 mL, $8.90 \mathrm{mmol})$, isobutyl chloroformate $(0.54 \mathrm{~mL}, 4.13 \mathrm{mmol})$ and $15 \mathrm{a}(0.90 \mathrm{~g}, 3.34 \mathrm{mmol})$ gave $21 \mathrm{c}$ as a white solid $(1.16 \mathrm{~g}, 87 \%) ; R_{f}=0.05$ ( $n$-hexane 3: EtOAc 1$) ;{ }^{1} \mathrm{H}$ NMR $\left(400 \mathrm{MHz}, \mathrm{CDCl}_{3}\right) \delta 7.48(\mathrm{~d}, J=8.2$ $\mathrm{Hz}, 2$ ArH), 7.36-7.41 (m, 1 ArH), 7.20-7.32 (m, 4 ArH), 6.10 (br s, C(O)NH), 4.98 (br s, $\mathrm{C}(\mathrm{O}) \mathrm{ONH}), 3.96\left(\mathrm{q}, J=7.0 \mathrm{~Hz}\right.$, chiral-H), 3.50-3.66 (m, $\left.\mathrm{NHCH}_{2} \mathrm{CH}_{2}\right), 2.88(\mathrm{t}, J=7.0 \mathrm{~Hz}$, $\left.\mathrm{NHCH}_{2} \mathrm{CH}_{2}\right), 1.56-1.92\left(\mathrm{~m}, \mathrm{CH}_{2} \mathrm{CH}_{3}\right), 1.45\left(\mathrm{~s}, \mathrm{C}\left(\mathrm{CH}_{3}\right)_{3}\right), 0.93\left(\mathrm{t}, J=7.4 \mathrm{~Hz}, \mathrm{CH}_{2} \mathrm{CH}_{3}\right)$.

tert-butyl(1-((2-(3',4'-dichloro-[1,1'-biphenyl]-4-yl)ethyl)amino)-1-oxobutan-2yl)carbamate (21d)

Using Method J, 8e (0.32 g, $1.57 \mathrm{mmol}), N$-methylmorpholine ( $0.43 \mathrm{~mL}, 3.93 \mathrm{mmol})$, isobutyl chloroformate $(0.27 \mathrm{~mL}, 2.05 \mathrm{mmol})$ and $15 \mathrm{a}(0.50 \mathrm{~g}, 1.65 \mathrm{mmol})$ gave $21 \mathrm{~d}$ as a white solid (0.66 g, 93\%): $R_{f}=0.05$ ( $n$-hexane $3:$ EtOAc 1); ${ }^{1} \mathrm{H}$ NMR $\left(400 \mathrm{MHz}, \mathrm{CDCl}_{3}\right) \delta 7.65(\mathrm{~s}, 1$ ArH), 7.47-7.50 (m, 3 ArH), 7.39 (d, $J=8.2 \mathrm{~Hz}, 1 \mathrm{ArH}), 7.28$ (s, 2 ArH), 6.07 (br s, C(O)NH), 4.94 (br s, C(O)ONH), 3.93 (d, $J=6.6 \mathrm{~Hz}$, chiral-H), 3.50-3.62 (m, $\left.\mathrm{NHCH}_{2} \mathrm{CH}_{2}\right), 2.86$ (t, $J=$ $\left.6.9 \mathrm{~Hz}, \mathrm{NHCH}_{2} \mathrm{CH}_{2}\right), 1.55-1.88\left(\mathrm{~m}, \mathrm{CH}_{2} \mathrm{CH}_{3}\right), 1.43\left(\mathrm{~s}, \mathrm{C}\left(\mathrm{CH}_{3}\right)_{3}\right), 0.91$ (t, J=7.4 Hz, $\left.\mathrm{CH}_{2} \mathrm{CH}_{3}\right)$.

tert-butyl(1-oxo-1-((2-(4'-(trifluoromethyl)-[1,1'-biphenyl]-4-yl)ethyl)amino)pentan-2yl)carbamate (21e)

Using Method J, 8a (0.55 g, $2.53 \mathrm{mmol}), N$-methylmorpholine ( $0.69 \mathrm{~mL}, 6.31 \mathrm{mmol})$, isobutyl chloroformate $(0.43 \mathrm{~mL}, 3.28 \mathrm{mmol})$ and $\mathbf{1 5 b}(0.80 \mathrm{~g}, 2.65 \mathrm{mmol})$ gave 21e as a white solid $(1.05 \mathrm{~g}, 89 \%): R_{f}=0.20$ ( $n$-hexane $3:$ EtOAc 1$) ;{ }^{1} \mathrm{H}$ NMR $\left(300 \mathrm{MHz}, \mathrm{CDCl}_{3}\right) \delta 7.67(\mathrm{~s}, 4$ ArH), 7.41 (dd, $J=8.1 \mathrm{~Hz}, 63.6 \mathrm{~Hz}, 4 \mathrm{ArH}), 6.36$ (br s, C(O)NH), 5.05-5.07 (m, C(O)ONH), 4.00-4.05 (m, chiral-H), 3.45-3.66 (m, $\left.\mathrm{NHCH}_{2} \mathrm{CH}_{2}\right), 2.87$ (t, $\left.J=7.1 \mathrm{~Hz}, \mathrm{NHCH}_{2} \mathrm{CH}_{2}\right), 1.50$ $1.83\left(\mathrm{~m}, \mathrm{CH}_{2} \mathrm{CH}_{2} \mathrm{CH}_{3}\right), 1.42\left(\mathrm{~s}, \mathrm{C}\left(\mathrm{CH}_{3}\right)_{3}\right), 1.28-1.39\left(\mathrm{~m}, \mathrm{CH}_{2} \mathrm{CH}_{2} \mathrm{CH}_{3}\right), 0.90$ (t, $J=7.2 \mathrm{~Hz}$, $\left.\mathrm{CH}_{2} \mathrm{CH}_{2} \mathrm{CH}_{3}\right) ;{ }^{13} \mathrm{C}$ NMR (75 MHz, $\left.\mathrm{CDCl}_{3}\right) \delta 171.3(\mathbf{C}(\mathrm{O}) \mathrm{NH}), 155.7(\mathbf{C}(\mathrm{O}) \mathrm{ONH}), 144.4$, 139.0, 138.0, 129.4, 127.5, 127.2, $125.7\left(\mathrm{q}, J_{\mathrm{C}-\mathrm{F}}=3.7 \mathrm{~Hz}\right), 124.3\left(\mathrm{q}, J_{\mathrm{C}-\mathrm{F}}=270.2 \mathrm{~Hz}, \mathbf{C F}_{3}\right)$ (ArC), $80.0\left(\mathbf{C}\left(\mathrm{CH}_{3}\right)_{3}\right), 54.5$ (chiral-C), $40.6\left(\mathrm{NHCH}_{2} \mathrm{CH}_{2}\right), 35.4\left(\mathrm{NHCH}_{2} \mathrm{CH}_{2}\right), 34.8$ $\left(\mathrm{CH}_{2} \mathbf{C H}_{2} \mathrm{CH}_{3}\right), 28.3\left(\mathrm{C}\left(\mathrm{CH}_{3}\right)_{3}\right), 18.9\left(\mathrm{CH}_{2} \mathrm{CH}_{2} \mathrm{CH}_{3}\right), 13.8\left(\mathrm{CH}_{2} \mathrm{CH}_{2} \mathbf{C H}_{3}\right)$.

\section{tert-butyl(1-oxo-1-((2-(4'-(trifluoromethoxy)-[1,1'-biphenyl]-4-yl)ethyl)amino)pentan-2-} yl)carbamate (21f)

Using Method J, 8c (0.33 g, $1.50 \mathrm{mmol}), N$-methylmorpholine $(0.41 \mathrm{~mL}, 3.75 \mathrm{mmol})$, isobutyl chloroformate $(0.25 \mathrm{~mL}, 1.95 \mathrm{mmol})$ and $\mathbf{1 5 b}(0.50 \mathrm{~g}, 1.57 \mathrm{mmol})$ gave $21 \mathbf{f}$ as a white solid $(0.70 \mathrm{~g}, 98 \%): R_{f}=0.12(n$-hexane $3:$ EtOAc 1$) ;{ }^{1} \mathrm{H}$ NMR $\left(400 \mathrm{MHz}, \mathrm{DMSO}-d_{6}\right) \delta 7.88(\mathrm{t}, J$ $=5.5 \mathrm{~Hz}, \mathrm{C}(\mathrm{O}) \mathrm{NH}), 7.74-7.77$ (m, $2 \mathrm{ArH}), 7.47-7.50$ (m, $3 \mathrm{ArH}), 7.59$ (d, $J=8.2 \mathrm{~Hz}, 2 \mathrm{ArH})$, 7.44 (d, $J=8.2 \mathrm{~Hz}, 2 \mathrm{ArH}), 7.32$ (d, $J=8.2 \mathrm{~Hz}, 2 \mathrm{ArH}), 6.74$ (d, $J=8.2 \mathrm{~Hz}, \mathrm{C}(\mathrm{O}) \mathrm{ONH}), 3.86$ 
(q, $J=5.7 \mathrm{~Hz}, 8.2 \mathrm{~Hz}$, chiral-H), 3.26-3.40 (m, $\left.\mathrm{NHCH}_{2} \mathrm{CH}_{2}\right), 2.76\left(\mathrm{t}, J=7.0 \mathrm{~Hz}, \mathrm{NHCH}_{2} \mathrm{CH}_{2}\right)$, 1.37-1.52 (m, $\left.\mathrm{CH}_{2} \mathrm{CH}_{2} \mathrm{CH}_{2} \mathrm{CH}_{3}\right), 1.37$ (s, $\left.\mathrm{C}\left(\mathrm{CH}_{3}\right)_{3}\right), 1.17-1.25\left(\mathrm{~m}, \mathrm{CH}_{2} \mathrm{CH}_{2} \mathrm{CH}_{2} \mathrm{CH}_{3}\right), 0.81$ (t, $\left.J=7.3 \mathrm{~Hz}, \mathrm{CH}_{2} \mathrm{CH}_{2} \mathrm{CH}_{2} \mathrm{CH}_{3}\right)$.

\section{tert-butyl(1-((2-(3',4'-difluoro-[1,1'-biphenyl]-4-yl)ethyl)amino)-1-oxopentan-2-} yl)carbamate (21g)

Using Method J, 8d (0.50 g, $2.29 \mathrm{mmol}), N$-methylmorpholine $(0.70 \mathrm{~mL}, 6.40 \mathrm{mmol})$, isobutyl chloroformate $(0.39 \mathrm{~mL}, 2.97 \mathrm{mmol})$ and $\mathbf{1 5 b}(0.80 \mathrm{~g}, 2.40 \mathrm{mmol})$ gave $21 \mathrm{~g}$ as a white solid $(0.97 \mathrm{~g}, 98 \%): R_{f}=0.10$ ( $n$-hexane $3:$ EtOAc 1$) ;{ }^{1} \mathrm{H}$ NMR $\left(400 \mathrm{MHz}, \mathrm{CDCl}_{3}\right) \delta 7.46(\mathrm{~d}, J=$ 7.9 Hz, 2 ArH), 7.33-7.38 (m, 1 ArH), 7.17-7.27 (m, 4 ArH), 6.11 (s, C(O)NH), 4.92 (br s, $\mathrm{C}(\mathrm{O}) \mathrm{ONH}$ ), 3.99 (q, $J=7.1 \mathrm{~Hz}$, chiral-H), 3.47-3.63 (m, $\left.\mathrm{NHCH}_{2} \mathrm{CH}_{2}\right), 2.85$ (t, $J=7.0 \mathrm{~Hz}$, $\left.\mathrm{NHCH}_{2} \mathrm{CH}_{2}\right), 1.48-1.82\left(\mathrm{~m}, \mathrm{CH}_{2} \mathrm{CH}_{2} \mathrm{CH}_{3}\right), 1.42\left(\mathrm{~s}, \mathrm{C}\left(\mathrm{CH}_{3}\right)_{3}\right), 1.26-1.36\left(\mathrm{~m}, \mathrm{CH}_{2} \mathrm{CH}_{2} \mathrm{CH}_{3}\right)$, $0.90\left(\mathrm{t}, J=7.3 \mathrm{~Hz}, \mathrm{CH}_{2} \mathrm{CH}_{2} \mathrm{CH}_{3}\right)$.

\section{tert-butyl(1-((2-(3',4'-dichloro-[1,1'-biphenyl]-4-yl)ethyl)amino)-1-oxopentan-2-} yl)carbamate $(21 \mathrm{~h})$

Using Method J, 8e (0.66 g, $3.02 \mathrm{mmol}), N$-methylmorpholine $(0.83 \mathrm{~mL}, 7.55 \mathrm{mmol})$, isobutyl chloroformate $(0.51 \mathrm{~mL}, 3.92 \mathrm{mmol})$ and $\mathbf{1 5 b}(0.96 \mathrm{~g}, 3.17 \mathrm{mmol})$ gave $\mathbf{2 1 h}$ as a white solid $(1.17 \mathrm{~g}, 83 \%): R_{f}=0.10(n$-hexane $3:$ EtOAc 1$) ;{ }^{1} \mathrm{H}$ NMR $\left(400 \mathrm{MHz}, \mathrm{DMSO}-d_{6}\right) \delta 7.90(\mathrm{~s}, 1$ ArH), 7.86 (s, C(O)NH), 7.63-7.71 (m, 4 ArH), 7.31 (d, J=7.7 Hz, 2 ArH), 6.73 (d, $J=7.9$ $\mathrm{Hz}, \mathrm{C}(\mathrm{O}) \mathrm{ONH}), 3.85$ (d, $J=6.1 \mathrm{~Hz}$, chiral-H), 3.26-3.37 (m, $\left.\mathrm{NHCH}_{2} \mathrm{CH}_{2}\right), 2.76(\mathrm{~d}, J=6.6$ $\left.\mathrm{Hz}, \quad \mathrm{NHCH}_{2} \mathrm{CH}_{2}\right), 1.37-1.47\left(\mathrm{~m}, \mathrm{CH}_{2} \mathrm{CH}_{2} \mathrm{CH}_{3}\right), 1.37\left(\mathrm{~s}, \mathrm{C}\left(\mathrm{CH}_{3}\right)_{3}\right), 1.18-1.21$ (m, $\mathrm{CH}_{2} \mathrm{CH}_{2} \mathrm{CH}_{3}$ ), 0.80 (t, $J=7.0 \mathrm{~Hz}, \mathrm{CH}_{2} \mathrm{CH}_{2} \mathrm{CH}_{3}$ ); ${ }^{13} \mathrm{C} \mathrm{NMR}\left(75 \mathrm{MHz}, \mathrm{CDCl}_{3}\right) \delta 172.3$ $(\mathbf{C}(\mathrm{O}) \mathrm{NH}), 155.7(\mathbf{C}(\mathrm{O}) \mathrm{ONH}), 140.9,138.9,137.0,132.8,131.3,130.7,130.1,129.4,128.8$, 127.1, 126.2 (ArC), $80.0\left(\mathbf{C}\left(\mathrm{CH}_{3}\right)_{3}\right), 54.5$ (chiral-C), $40.5\left(\mathrm{NHCH}_{2} \mathrm{CH}_{2}\right), 35.4\left(\mathrm{NHCH}_{2} \mathrm{CH}_{2}\right)$, $34.7\left(\mathrm{CH}_{2} \mathrm{CH}_{2} \mathrm{CH}_{3}\right), 28.3\left(\mathrm{C}\left(\mathrm{CH}_{3}\right)_{3}\right), 18.9\left(\mathrm{CH}_{2} \mathrm{CH}_{2} \mathrm{CH}_{3}\right), 13.8\left(\mathrm{CH}_{2} \mathrm{CH}_{2} \mathrm{CH}_{3}\right)$.

\section{tert-butyl(1-oxo-1-((2-(4'-(trifluoromethyl)-[1,1'-biphenyl]-4-yl)ethyl)amino)hexan-2-} yl)carbamate (21i)

Using Method J, 8a (0.73 g, $3.16 \mathrm{~m} \mathrm{~mol}), N$-methylmorpholine $(0.87 \mathrm{~mL}, 7.89 \mathrm{mmol})$, isobutyl chloroformate $(0.53 \mathrm{~mL}, 4.10 \mathrm{mmol})$ and $\mathbf{1 5 c}(1.00 \mathrm{~g}, 3.31 \mathrm{mmol})$ gave $21 \mathbf{i}$ as a white solid $(1.34 \mathrm{~g}, 89 \%): R_{f}=0.13(n$-hexane $3:$ EtOAc 1$) ;{ }^{1} \mathrm{H}$ NMR $\left(400 \mathrm{MHz}, \mathrm{DMSO}-d_{6}\right) \delta 7.87(\mathrm{~d}, J$ $=8.2 \mathrm{~Hz}, 2 \operatorname{ArH}, \mathrm{C}(\mathrm{O}) \mathrm{NH}), 7.80(\mathrm{~d}, J=8.5 \mathrm{~Hz}, 2 \operatorname{ArH}), 7.66(\mathrm{~d}, J=8.1 \mathrm{~Hz}, 2 \operatorname{ArH}), 7.35(\mathrm{~d}, J$ $=8.1 \mathrm{~Hz}, 2 \mathrm{ArH}), 6.73(\mathrm{~d}, J=8.2 \mathrm{~Hz}, \mathrm{C}(\mathrm{O}) \mathrm{ONH}), 3.83(\mathrm{q}, J=5.6 \mathrm{~Hz}, 8.4 \mathrm{~Hz}$, chiral-H), 3.24$3.43\left(\mathrm{~m}, \mathrm{NHCH}_{2} \mathrm{CH}_{2}\right), 2.77$ (d, $\left.J=7.0 \mathrm{~Hz}, \mathrm{NHCH}_{2} \mathrm{CH}_{2}\right), 1.37-1.52\left(\mathrm{~m}, \mathrm{CH}_{2} \mathrm{CH}_{2} \mathrm{CH}_{2} \mathrm{CH}_{3}\right.$ ), 1.37 (s, $\left.\mathrm{C}\left(\mathrm{CH}_{3}\right)_{3}\right), 1.15-1.20\left(\mathrm{~m}, \mathrm{CH}_{2} \mathrm{CH}_{2} \mathrm{CH}_{2} \mathrm{CH}_{3}\right), 0.80$ (d, $\left.J=6.8 \mathrm{~Hz}, \mathrm{CH}_{2} \mathrm{CH}_{2} \mathrm{CH}_{2} \mathrm{CH}_{3}\right)$.

\section{tert-butyl(1-oxo-1-((4'-(trifluoromethoxy)-[1,1'-biphenyl]-4-ylamino)hexan-2-} yl)carbamate (21j)

Using Method J, 8c (0.46 g, $1.97 \mathrm{mmol}), N$-methylmorpholine (0.61 mL, $5.52 \mathrm{mmol})$, isobutyl chloroformate $(0.33 \mathrm{~mL}, 2.56 \mathrm{mmol})$ and $\mathbf{1 5 c}(0.60 \mathrm{~g}, 2.07 \mathrm{mmol})$ gave $\mathbf{2 1} \mathbf{j}$ as a white solid 
(0.64 g, 69\%): $R_{f}=0.39$ ( $n$-hexane $3:$ EtOAc 1); ${ }^{1} \mathrm{H}$ NMR (400 MHz, $\left.\mathrm{CDCl}_{3}\right) \delta 8.42$ (br s, $\mathrm{C}(\mathrm{O}) \mathrm{NH}), 7.62(\mathrm{~d}, J=8.5 \mathrm{~Hz}, 2 \mathrm{ArH}), 7.57$ (d, $J=8.6 \mathrm{~Hz}, 2 \mathrm{ArH}), 7.52(\mathrm{~d}, J=8.5 \mathrm{~Hz}, 2 \mathrm{ArH})$, 7.27-7.29 (m, 2 ArH), 5.06 (br s, C(O)ONH), 4.22 (d, $J=5.2 \mathrm{~Hz}$, chiral-H), 1.66-2.01 (m, $\left.\mathrm{CH}_{2} \mathrm{CH}_{2} \mathrm{CH}_{2} \mathrm{CH}_{3}\right), 1.50\left(\mathrm{~s}, \mathrm{C}\left(\mathrm{CH}_{3}\right)_{3}\right), 1.41-1.42\left(\mathrm{~m}, \mathrm{CH}_{2} \mathrm{CH}_{2} \mathrm{CH}_{2} \mathrm{CH}_{3}\right), 0.94$ (t, $J=6.8 \mathrm{~Hz}$, $\mathrm{CH}_{2} \mathrm{CH}_{2} \mathrm{CH}_{2} \mathrm{CH}_{3}$ ).

tert-butyl(1-((2-(3',4'-difluoro-[1,1'-biphenyl]-4-yl)ethyl)amino)-1-oxohexan-2yl)carbamate (21k)

Using Method J, 8d (1.26 g, $5.45 \mathrm{mmol}), N$-methylmorpholine (1.34 mL, $12.2 \mathrm{mmol})$, isobutyl chloroformate $(0.48 \mathrm{~mL}, 3.67 \mathrm{mmol})$ and $15 \mathrm{c}(0.80 \mathrm{~g}, 2.97 \mathrm{mmol})$ gave $21 \mathbf{k}$ as a white solid $(1.29 \mathrm{~g}, 97 \%) ; R_{f}=0.08$ ( $n$-hexane 3: EtOAc 1); ${ }^{1} \mathrm{H} \mathrm{NMR}\left(400 \mathrm{MHz}, \mathrm{CDCl}_{3}\right) \delta 7.48(\mathrm{~d}, J=8.1$ Hz, 2 ArH), 7.36-7.41 (m, 1 ArH), 7.20-7.30 (m, 4 ArH), 6.11 (br s, C(O)NH), 4.94 (br s, $\mathrm{C}(\mathrm{O}) \mathrm{ONH}), 3.96$ (q, $J=6.9 \mathrm{~Hz}, \quad$ chiral-H), 3.50-3.66 (m, $\left.\mathrm{NHCH}_{2} \mathrm{CH}_{2}\right), 2.88(\mathrm{t}, J=7.0 \mathrm{~Hz}$, $\left.\mathrm{NHCH}_{2} \mathrm{CH}_{2}\right), \quad 1.51-1.87$ (m, $\left.\mathrm{CH}_{2} \mathrm{CH}_{2} \mathrm{CH}_{2} \mathrm{CH}_{3}\right), 1.45$ (s, $\left.\mathrm{C}\left(\mathrm{CH}_{3}\right)_{3}\right), 1.31-1.37$ (m, $\left.\mathrm{CH}_{2} \mathrm{CH}_{2} \mathrm{CH}_{2} \mathrm{CH}_{3}\right), 0.90$ ( $\mathrm{t}, J=6.9 \mathrm{~Hz}, \mathrm{CH}_{2} \mathrm{CH}_{2} \mathrm{CH}_{2} \mathrm{CH}_{3}$ ).

\section{tert-butyl(1-((2-(3',4'-dichloro-[1,1'-biphenyl]-4-yl)ethyl)amino)-1-oxohexan-2-} yl)carbamate (211)

Using Method J, 8e (0.36 g, $1.57 \mathrm{mmol}), N$-methylmorpholine $(0.43 \mathrm{~mL}, 3.93 \mathrm{mmol})$, isobutyl chloroformate $(0.27 \mathrm{~mL}, 2.05 \mathrm{mmol})$ and $15 \mathrm{c}(0.50 \mathrm{~g}, 16.5 \mathrm{mmol})$ gave $21 \mathrm{l}$ as a white solid $(0.76 \mathrm{~g}, 100 \%): R_{f}=0.07$ ( $n$-hexane $3:$ EtOAc 1); ${ }^{1} \mathrm{H}$ NMR $\left(400 \mathrm{MHz}, \mathrm{CDCl}_{3}\right) \delta 7.65$ (s, 1 ArH), 7.34-7.49 (m, 4 ArH), 7.26-7.28 (m, $2 \mathrm{ArH}$, the peak was overlapped with the $\mathrm{CDCl}_{3}$ signals.), 6.07 (br s, C(O)NH), 4.91 (br s, C(O)ONH), 3.97 (d, J=5.6 Hz, chiral-H), 3.51-3.62 $\left(\mathrm{m}, \mathrm{NHCH}_{2} \mathrm{CH}_{2}\right), 2.86$ ( $\left.\mathrm{t}, J=6.7 \mathrm{~Hz}, \mathrm{NHCH}_{2} \mathrm{CH}_{2}\right), 1.51-1.81\left(\mathrm{~m}, \mathrm{CH}_{2} \mathrm{CH}_{2} \mathrm{CH}_{2} \mathrm{CH}_{3}\right), 1.42$ (s, $\left.\mathrm{C}\left(\mathrm{CH}_{3}\right)_{3}\right), 1.27\left(\mathrm{~d}, J=6.7 \mathrm{~Hz}, \mathrm{CH}_{2} \mathrm{CH}_{2} \mathrm{CH}_{2} \mathrm{CH}_{3}\right), 0.87-0.88\left(\mathrm{~m}, \mathrm{CH}_{2} \mathrm{CH}_{2} \mathrm{CH}_{2} \mathrm{CH}_{3}\right)$.

\section{tert-butylmethyl(1-oxo-1-((2-(4'-(trifluoromethyl)-[1,1'-biphenyl]-4- yl)ethyl)amino)butan-2-yl)carbamate (21m)}

Using Method J, 8a (0.62 g, $2.84 \mathrm{mmol}), N$-methylmorpholine $(0.87 \mathrm{~mL}, 7.95 \mathrm{mmol})$, isobutyl chloroformate $(0.48 \mathrm{~mL}, 3.69 \mathrm{mmol})$ and $\mathbf{1 6 a}(0.90 \mathrm{~g}, 2.98 \mathrm{mmol})$ gave $\mathbf{2 1} \mathbf{m}$ as a white solid (0.89 g, 67\%): $R_{f}=0.15$ ( $n$-hexane $3:$ EtOAc 1); ${ }^{1} \mathrm{H}$ NMR $\left(400 \mathrm{MHz}, \mathrm{CDCl}_{3}\right) \delta 7.68-7.73(\mathrm{~m}$, $4 \operatorname{ArH}), 7.56$ (d, $J=7.9$ Hz, 2 ArH), 7.29-7.32 (m, 2 ArH), 5.94-6.22 (m, C(O)NH), 4.45 (s, chiral-H), 3.57-3.64 (m, $\left.\mathrm{NHCH}_{2} \mathrm{CH}_{2}\right), 2.88$ (t, $\left.J=6.1 \mathrm{~Hz}, \mathrm{NHCH}_{2} \mathrm{CH}_{2}\right), 2.70\left(\mathrm{NCH}_{3}\right), 1.61-$ $1.99\left(\mathrm{~m}, \mathrm{CH}_{2} \mathrm{CH}_{3}\right), 1.45$ (s, $\left.\mathrm{C}\left(\mathrm{CH}_{3}\right)_{3}\right), 0.89$ (t, $\left.J=7.3 \mathrm{~Hz}, \mathrm{CH}_{2} \mathrm{CH}_{3}\right)$.

\section{tert-butylmethyl(1-oxo-1-((2-(4'-(trifluoromethoxy)-[1,1'-biphenyl]-4-} yl)ethyl)amino)butan-2-yl)carbamate (21n)

Using Method J, 8c (0.65 g, $3.00 \mathrm{mmol}), N$-methylmorpholine (0.92 mL, $8.99 \mathrm{mmol})$, isobutyl chloroformate $(0.51 \mathrm{~mL}, 3.90 \mathrm{mmol})$ and $16 \mathrm{a}(1.00 \mathrm{~g}, 3.15 \mathrm{mmol})$ gave $21 \mathrm{n}$ as a oil $(1.00 \mathrm{~g}$, 69\%): $R_{f}=0.19$ (n-hexane $3:$ EtOAc 1); $\left.{ }^{1} \mathrm{H} \mathrm{NMR} \mathrm{(400} \mathrm{MHz,} \mathrm{CDCl}_{3}\right) \delta 7.51-7.61$ (m, $\left.4 \mathrm{ArH}\right)$, 7.28-7.30 (m, 4 ArH), 5.98-6.26 (m, C(O)NH), 4.45 (s, chiral-H), 3.59-3.60 (m, $\left.\mathrm{NHCH}_{2} \mathrm{CH}_{2}\right)$, 
$2.87\left(\mathrm{~s}, \mathrm{NHCH}_{2} \mathrm{CH}_{2}\right), 2.69\left(\mathrm{NCH}_{3}\right), 1.62-1.99\left(\mathrm{~m}, \mathrm{CH}_{2} \mathrm{CH}_{3}\right), 1.45\left(\mathrm{~s}, \mathrm{C}\left(\mathrm{CH}_{3}\right)_{3}\right), 0.87-0.91(\mathrm{~m}$, $\mathrm{CH}_{2} \mathrm{CH}_{3}$ ).

tert-butyl(1-((3',4'-dichloro-[1,1'-biphenyl]-4-yl)ethyl)amino)-1-oxobutan-2yl)(methyl)carbamate (210)

Using Method J, 8e (0.82 g, $3.78 \mathrm{~m} \mathrm{~mol}), N$-methylmorpholine (1.04 mL, $9.44 \mathrm{mmol})$, isobutyl chloroformate $(0.64 \mathrm{~m} \mathrm{~L}, 4.91 \mathrm{mmol})$ and 16a $(1.20 \mathrm{~g}, 3.97 \mathrm{mmol})$ gave 210 as a oil $(1.38 \mathrm{~g}$, 79\%): $R_{f}=0.22$ ( $n$-hexane 3 : EtOAc 1); ${ }^{1} \mathrm{H}$ NMR (400 MHz, $\left.\mathrm{CDCl}_{3}\right) \delta 7.66(\mathrm{~d}, J=2.0 \mathrm{~Hz}, 1$ ArH), 7.45-7.53 (m, 3 ArH), 7.41 (dd, $J=2.1 \mathrm{~Hz}, 6.3 \mathrm{~Hz}, 1$ ArH), 7.27-7.29 (m, 2 ArH), 5.90-6.26 (m, C(O)NH), 4.44 (s, chiral-H), 3.55-3.64 (m, $\left.\mathrm{NHCH}_{2} \mathrm{CH}_{2}\right), 2.87(\mathrm{t}, J=6.7 \mathrm{~Hz}$, $\left.\mathrm{NHCH}_{2} \mathrm{CH}_{2}\right), 2.70\left(\mathrm{NCH}_{3}\right), 1.61-1.98\left(\mathrm{~m}, \mathrm{CH}_{2} \mathrm{CH}_{3}\right), 1.45\left(\mathrm{~s}, \mathrm{C}\left(\mathrm{CH}_{3}\right)_{3}\right), 0.88(\mathrm{t}, J=7.4 \mathrm{~Hz}$, $\mathrm{CH}_{2} \mathrm{CH}_{3}$ ).

tert-butylmethyl(1-oxo-1-((2-(4'-(trifluoromethyl)-[1,1'-biphenyl]-4yl)ethyl)amino)pentan-2-yl)carbamate (21p)

Using Method J, 8a (0.58 g, $2.53 \mathrm{mmol}), N$-methylmorpholine $(0.69 \mathrm{~mL}, 6.31 \mathrm{mmol})$, isobutyl chloroformate $(0.43 \mathrm{~mL}, 3.28 \mathrm{mmol})$ and $16 \mathrm{~b}(0.80 \mathrm{~g}, 2.65 \mathrm{mmol})$ gave $21 \mathrm{p}$ as a oil $(1.19 \mathrm{~g}$, 98\%): $R_{f}=0.26(n$-hexane $3:$ EtOAc 1$) ;{ }^{1} \mathrm{H} \mathrm{NMR}\left(300 \mathrm{MHz}, \mathrm{CDCl}_{3}\right) \delta 7.64-7.70(\mathrm{~m}, 4 \mathrm{ArH})$, 7.41 (dd, $J=8.1 \mathrm{~Hz}, 67.4 \mathrm{~Hz}, 4 \mathrm{ArH}), 6.21$ (br s, C(O)NH), 5.95 (br s, C(O)ONH), 4.52 (br s, chiral-H), 3.54-3.63 (m, $\left.\mathrm{NHCH}_{2} \mathrm{CH}_{2}\right), 2.86$ (t, $\left.J=6.8 \mathrm{~Hz}, \mathrm{NHCH}_{2} \mathrm{CH}_{2}\right), 1.55-1.91$ (m, $\left.\mathrm{CH}_{2} \mathrm{CH}_{2} \mathrm{CH}_{3}\right), 1.42\left(\mathrm{~s}, \mathrm{C}\left(\mathrm{CH}_{3}\right)_{3}\right), 1.23-1.27\left(\mathrm{~m}, \mathrm{CH}_{2} \mathrm{CH}_{2} \mathrm{CH}_{3}\right), 0.88-0.95\left(\mathrm{~m}, \mathrm{CH}_{2} \mathrm{CH}_{2} \mathrm{CH}_{3}\right)$; ${ }^{13} \mathrm{C}$ NMR $\left(75 \mathrm{MHz}, \mathrm{CDCl}_{3}\right) \delta 171.2(\mathbf{C}(\mathrm{O}) \mathrm{NH}), 156.7(\mathbf{C}(\mathrm{O}) \mathrm{ONH}), 144.4,139.0,138.0,129.4$, 127.5, 127.2, 125.7 (q, $\left.J_{\mathrm{C}-\mathrm{F}}=3.7 \mathrm{~Hz}\right), 124.3\left(\mathrm{q}, J_{\mathrm{C}-\mathrm{F}}=270.0 \mathrm{~Hz}, \mathbf{C F}_{3}\right)(\mathrm{ArC}), 80.5\left(\mathbf{C}\left(\mathrm{CH}_{3}\right)_{3}\right)$, 58.0 (chiral-C), $40.4\left(\mathrm{NHCH}_{2} \mathrm{CH}_{2}\right), 35.5\left(\mathrm{NHCH}_{2} \mathrm{CH}_{2}\right), 31.6\left(\mathrm{CH}_{2} \mathrm{CH}_{2} \mathrm{CH}_{3}\right), 29.8\left(\mathrm{NCH}_{3}\right)$, $28.4\left(\mathrm{C}\left(\mathrm{CH}_{3}\right)_{3}\right), 19.2\left(\mathrm{CH}_{2} \mathrm{CH}_{2} \mathrm{CH}_{3}\right), 13.7\left(\mathrm{CH}_{2} \mathrm{CH}_{2} \mathbf{C H}_{3}\right)$.

tert-butylmethyl(1-oxo-1-((2-(4'-(trifluoromethoxy)-[1,1'-biphenyl]-4yl)ethyl)amino)pentan-2-yl)carbamate (21q)

Using Method J, 8c (0.25 g, $1.08 \mathrm{mmol}), N$-methylmorpholine $(0.30 \mathrm{~mL}, 2.69 \mathrm{mmol})$, isobutyl chloroformate $(0.18 \mathrm{~mL}, 1.40 \mathrm{mmol})$ and $\mathbf{1 6 b}(0.36 \mathrm{~g}, 1.13 \mathrm{mmol})$ gave $\mathbf{2 1 q}$ as a white solid $(0.32 \mathrm{~g}, 60 \%): R_{f}=0.18$ ( $n$-hexane $3:$ EtOAc 1$) ;{ }^{1} \mathrm{H}$ NMR $\left(400 \mathrm{MHz}, \mathrm{DMSO}-d_{6}\right) \delta 7.89$ (br s, $\mathrm{C}(\mathrm{O}) \mathrm{NH}), 7.76(\mathrm{~d}, J=8.4 \mathrm{~Hz}, 2 \mathrm{ArH}), 7.59$ (d, $J=7.8 \mathrm{~Hz}, 2 \mathrm{ArH}), 7.44$ (d, $J=8.2 \mathrm{~Hz}, 2 \mathrm{ArH})$, $7.30(\mathrm{~d}, J=8.2 \mathrm{~Hz}, 2 \mathrm{ArH}), 4.39\left(\mathrm{~d}, J=73.1 \mathrm{~Hz}\right.$, chiral-H), $3.34\left(\mathrm{~s}, \mathrm{NHCH}_{2} \mathrm{CH}_{2}\right.$, the peak was overlapped with the $\mathrm{H}_{2} \mathrm{O}$ signals.), 2.77 ( $\left.\mathrm{s}, \mathrm{NHCH}_{2} \mathrm{CH}_{2}\right), 2.66$ (s, $\left.\mathrm{NCH}_{3}\right), 1.53-1.68$ (m, $\left.\mathrm{CH}_{2} \mathrm{CH}_{2} \mathrm{CH}_{3}\right), 1.39$ (s, $\left.\mathrm{C}\left(\mathrm{CH}_{3}\right)_{3}\right), 1.16-1.23$ (m, $\left.\mathrm{CH}_{2} \mathrm{CH}_{2} \mathrm{CH}_{3}\right), 0.89$ (d, $J=6.5 \mathrm{~Hz}$, $\mathrm{CH}_{2} \mathrm{CH}_{2} \mathrm{CH}_{3}$ ).

\section{tert-butyl(1-((3',4'-dichloro-[1,1'-biphenyl]-4-yl)ethyl)amino)-1-oxopentan-2-} yl)(methyl)carbamate (21r)

Using Method J, 8e $(0.35 \mathrm{~g}, 1.51 \mathrm{~m} \mathrm{~mol}), N$-methylmorpholine $(0.33 \mathrm{~mL}, 3.03 \mathrm{mmol})$, isobutyl chloroformate $(0.26 \mathrm{~m} \mathrm{~L}, 1.97 \mathrm{mmol})$ and $\mathbf{1 6 b}(0.48 \mathrm{~g}, 1.59 \mathrm{mmol})$ gave $\mathbf{2 1 r}$ as a oil $(0.69 \mathrm{~g}$, 
95\%): $R_{f}=0.33$ (n-hexane $3:$ EtOAc 1); ${ }^{1} \mathrm{H} \mathrm{NMR}\left(400 \mathrm{MHz}, \mathrm{CDCl}_{3}\right) \delta 7.46-7.64$ (m, $\left.4 \mathrm{ArH}\right)$, 7.35-7.40 (m, 2 ArH), 7.25-7.27 (m, 2 ArH), 5.95-6.25 (m, C(O)NH), 4.51 (br s, chiral-H), 3.53-3.60 (m, $\left.\mathrm{NHCH}_{2} \mathrm{CH}_{2}\right), 2.84\left(\mathrm{t}, J=6.6 \mathrm{~Hz}, \mathrm{NHCH}_{2} \mathrm{CH}_{2}\right), 2.67\left(\mathrm{~s}, \mathrm{NCH}_{3}\right), 1.57-1.90(\mathrm{~m}$, $\left.\mathrm{CH}_{2} \mathrm{CH}_{2} \mathrm{CH}_{3}\right), 1.42\left(\mathrm{~s}, \mathrm{C}\left(\mathrm{CH}_{3}\right)_{3}\right), 1.22-1.32\left(\mathrm{~m}, \mathrm{CH}_{2} \mathrm{CH}_{2} \mathrm{CH}_{3}\right), 0.95$ (t, $J=7.3 \mathrm{~Hz}$, $\left.\mathrm{CH}_{2} \mathrm{CH}_{2} \mathrm{CH}_{3}\right) ;{ }^{13} \mathrm{C}$ NMR $\left(75 \mathrm{MHz}, \mathrm{CDCl}_{3}\right) \delta 171.2(\mathbf{C}(\mathrm{O}) \mathrm{NH}), 156.8(\mathbf{C}(\mathrm{O}) \mathrm{ONH}), 140.9$, 139.4, 138.9, 137.0, 132.8, 131.3, 130.7, 129.4, 128.8, 127.1, $126.2(\mathrm{ArC}), 80.4\left(\mathbf{C}\left(\mathrm{CH}_{3}\right)_{3}\right)$, 57.9 (chiral-C), $40.3\left(\mathrm{NHCH}_{2} \mathrm{CH}_{2}\right), 35.4\left(\mathrm{NHCH}_{2} \mathrm{CH}_{2}\right), 31.6\left(\mathrm{CH}_{2} \mathrm{CH}_{2} \mathrm{CH}_{3}\right), 29.8\left(\mathrm{NCH}_{3}\right)$, $28.3\left(\mathrm{C}\left(\mathrm{CH}_{3}\right)_{3}\right), 19.2\left(\mathrm{CH}_{2} \mathrm{CH}_{2} \mathrm{CH}_{3}\right), 13.7\left(\mathrm{CH}_{2} \mathrm{CH}_{2} \mathbf{C H}_{3}\right)$.

tert-butylmethyl(1-oxo-1-((2-(4'-(trifluoromethyl)-[1,1'-biphenyl]-4yl)ethyl)amino)hexan-2-yl)carbamate (21s)

Using Method J, 8a (0.70 g, $2.84 \mathrm{mmol}), N$-methylmorpholine $(0.87 \mathrm{~mL}, 7.95 \mathrm{mmol})$, isobutyl chloroformate $(0.48 \mathrm{~mL}, 3.69 \mathrm{mmol})$ and $16 \mathrm{c}(0.90 \mathrm{~g}, 2.98 \mathrm{mmol})$ gave $21 \mathrm{~s}$ as a pale yellow solid (0.88 g, 63\%): $R_{f}=0.32$ ( $n$-hexane $3:$ EtOAc 1$) ;{ }^{1} \mathrm{H}$ NMR (400 MHz, DMSO- $\left.d_{6}\right) \delta 7.87$ (d, $J=8.3 \mathrm{~Hz}, 2 \mathrm{ArH}, \mathrm{C}(\mathrm{O}) \mathrm{NH}), 7.80$ (d, $J=8.4 \mathrm{~Hz}, 2 \mathrm{ArH}), 7.66$ (d, $J=8.2 \mathrm{~Hz}, 2 \mathrm{ArH}), 7.33$ (d, $J=8.1 \mathrm{~Hz}, 2 \mathrm{ArH}), 4.27-4.47$ (m, chiral-H), 3.32-3.33 (m, $\mathrm{NHCH}_{2} \mathrm{CH}_{2}$, the peak was overlapped with the $\mathrm{H}_{2} \mathrm{O}$ signals.), $2.78\left(\mathrm{t}, J=6.8 \mathrm{~Hz}, \mathrm{NHCH}_{2} \mathrm{CH}_{2}\right), 2.66\left(\mathrm{NCH}_{3}\right), 1.53-1.71(\mathrm{~m}$, $\left.\mathrm{CH}_{2} \mathrm{CH}_{2} \mathrm{CH}_{2} \mathrm{CH}_{3}\right), \quad 1.39$ (s, $\left.\mathrm{C}\left(\mathrm{CH}_{3}\right)_{3}\right), \quad 1.24-1.28 \quad\left(\mathrm{~m}, \quad \mathrm{CH}_{2} \mathrm{CH}_{2} \mathrm{CH}_{2} \mathrm{CH}_{3}\right), \quad 1.12$ (s, $\left.\mathrm{CH}_{2} \mathrm{CH}_{2} \mathrm{CH}_{2} \mathrm{CH}_{3}\right), 0.84-0.89\left(\mathrm{~m}, \mathrm{CH}_{2} \mathrm{CH}_{2} \mathrm{CH}_{2} \mathrm{CH}_{3}\right)$.

\section{tert-butylmethyl(1-oxo-1-((2-(4'-(trifluoromethoxy)-[1,1'-biphenyl]-4-} yl)ethyl)amino)hexan-2-yl)carbamate (21t)

Using Method J, 8c (0.74 g, $3.00 \mathrm{mmol}), N$-methylmorpholine (0.92 mL, $8.99 \mathrm{mmol})$, isobutyl chloroformate $(0.51 \mathrm{~mL}, 3.90 \mathrm{mmol})$ and $\mathbf{1 6 c}(1.00 \mathrm{~g}, 3.15 \mathrm{mmol})$ gave $21 \mathrm{t}$ as a oil $(0.92 \mathrm{~g}$, 60\%): $R_{f}=0.29(n$-hexane $3:$ EtOAc 1$) ;{ }^{1} \mathrm{H} \mathrm{NMR}\left(400 \mathrm{MHz}, \mathrm{DMSO}-d_{6}\right) \delta 7.88(\mathrm{br} \mathrm{s}, \mathrm{C}(\mathrm{O}) \mathrm{NH})$, 7.74-7.77 (m, 2 ArH), 7.59 (d, $J=8.2 \mathrm{~Hz}, 2 \operatorname{ArH}), 7.44$ (d, $J=8.0 \mathrm{~Hz}, 2 \operatorname{ArH}), 7.30$ (d, $J=$ $8.1 \mathrm{~Hz}, 2 \mathrm{ArH}), 4.28-4.47$ (m, chiral-H), 3.30-3.34 (m, $\mathrm{NHCH}_{2} \mathrm{CH}_{2}$, the peak was overlapped with the $\mathrm{H}_{2} \mathrm{O}$ signals.), $2.77\left(\mathrm{t}, J=6.7 \mathrm{~Hz}, \mathrm{NHCH}_{2} \mathrm{CH}_{2}\right), 2.66\left(\mathrm{NCH}_{3}\right), 1.53-1.72(\mathrm{~m}$, $\left.\mathrm{CH}_{2} \mathrm{CH}_{2} \mathrm{CH}_{2} \mathrm{CH}_{3}\right), 1.39$ (s, $\left.\mathrm{C}\left(\mathrm{CH}_{3}\right)_{3}\right), 1.12-1.29$ (m, $\left.\mathrm{CH}_{2} \mathrm{CH}_{2} \mathrm{CH}_{2} \mathrm{CH}_{3}\right), 0.84-0.90$ (m, $\mathrm{CH}_{2} \mathrm{CH}_{2} \mathrm{CH}_{2} \mathrm{CH}_{3}$ ).

\section{tert-butyl(1-((2-(3',4'-dichloro-[1,1'-biphenyl]-4-yl)ethyl)amino)-1-oxohexan-2- yl)(methyl)carbamate (21u)}

Using Method J, 8e $(0.41 \mathrm{~g}, 1.67 \mathrm{mmol}), N$-methylmorpholine $(0.46 \mathrm{~mL}, 4.18 \mathrm{mmol})$, isobutyl chlorof ormate $(0.28 \mathrm{~mL}, 2.17 \mathrm{mmol})$ and $16 \mathbf{c}(0.38 \mathrm{~g}, 1.26 \mathrm{mmol})$ gave $21 \mathrm{u}$ as a oil $(0.60 \mathrm{~g}$, 97\%): $R_{f}=0.30$ ( $n$-hexane $3:$ EtOAc 1$) ;{ }^{1} \mathrm{H}$ NMR $\left(400 \mathrm{MHz}, \mathrm{DMSO}-d_{6}\right) \delta 7.89(\mathrm{~d}, J=1.9 \mathrm{~Hz}$, $1 \mathrm{ArH}, \mathrm{C}(\mathrm{O}) \mathrm{NH}), 7.62-7.71$ (m, 4 ArH), 7.30 (d, $J=8.1 \mathrm{~Hz}, 2 \mathrm{ArH}), 4.37$ (d, J=8.0 Hz, chiralH), 3.32-3.35 (m, $\left.\mathrm{NHCH}_{2} \mathrm{CH}_{2}\right), 2.77\left(\mathrm{t}, J=6.5 \mathrm{~Hz}, \mathrm{NHCH}_{2} \mathrm{CH}_{2}\right), 2.66$ (s, $\left.\mathrm{NCH}_{3}\right), 1.53-1.71$ $\left(\mathrm{m}, \mathrm{CH}_{2} \mathrm{CH}_{2} \mathrm{CH}_{2} \mathrm{CH}_{3}\right), 1.39$ ( $\left.\mathrm{s}, \mathrm{C}\left(\mathrm{CH}_{3}\right)_{3}\right), 1.12-1.28\left(\mathrm{~m}, \mathrm{CH}_{2} \mathrm{CH}_{2} \mathrm{CH}_{2} \mathrm{CH}_{3}\right), 0.84$ (br s, $\mathrm{CH}_{2} \mathrm{CH}_{2} \mathrm{CH}_{2} \mathrm{CH}_{3}$ ). 


\subsection{Synthesis of 23}

tert-butyl 2-((2-(4'-(trifluoromethyl)-[1,1'-biphenyl]-4-yl)ethyl)carbamoyl)pyrrolidine-1carboxylate (23a)

Using Method J, to a solution of (tert-butoxycarbonyl)proline ( $0.80 \mathrm{~g}, 3.72 \mathrm{mmol})$, NMM (0.82 mL. $7.43 \mathrm{mmol})$, IBCF (0.63 mL, $4.83 \mathrm{mmol}), 8 \mathrm{a}(1.18 \mathrm{~g}, 3.90 \mathrm{mmol})$ in THF anhydrous (37 $\mathrm{mL}$ ) gave $23 \mathrm{a}$ as a white solid (1.48 g, 85\%); $R_{f}=0.36$ (EtOAc $2: \mathrm{n}$-hexane 1$) ;{ }^{1} \mathrm{H}$ NMR (300 $\left.\mathrm{MHz}, \mathrm{CDCl}_{3}\right) \delta 7.68(\mathrm{~s}, 6 \mathrm{ArH}), 7.54(\mathrm{~d}, J=8.0 \mathrm{~Hz}, 2 \mathrm{ArH}), 7.32-7.27$ (m, $\left.2 \mathrm{ArH}\right), 6.98-6.14$ (m, C(O)NH), 4.25 (br s, C(O)CH), 3.56-3.52 (m, $\mathrm{NHCH}_{2} \mathrm{CH}_{2}$ ), 3.35 (br s, C(O)NCH ), 2.95$2.82\left(\mathrm{~m}, \mathrm{NHCH}_{2} \mathrm{CH}_{2}\right), 2.35-1.85\left(\mathrm{~m}, \mathrm{CH}_{2} \mathrm{CH}_{2} \mathrm{CH}\right), 1.42$ (br s, $\left.\mathrm{C}\left(\mathrm{CH}_{3}\right)_{3}\right) ;{ }^{13} \mathrm{C} \mathrm{NMR}(75 \mathrm{MHz}$, $\left.\mathrm{CDCl}_{3}\right) \delta 172.2(\mathbf{C}(\mathrm{O}) \mathrm{NH}), 155.8(\mathbf{C}(\mathrm{O}) \mathrm{ONH}), 144.4,139.1,138.0,129.4,129.3\left(\mathrm{q}, J_{\mathrm{C}-\mathrm{F}}=32.7\right.$ $\mathrm{Hz}), 127.4,127.2,125.7$ (q, $\left.J_{\mathrm{C}-\mathrm{F}}=3.7 \mathrm{~Hz}\right), 124.3$ (q, $\left.J_{\mathrm{C}-\mathrm{F}}=270.1 \mathrm{~Hz}, \mathbf{C F}_{3}\right)(\mathrm{ArC}), 80.4$ $\left(\mathbf{C}\left(\mathrm{CH}_{3}\right)_{3}\right), 61.2(\mathbf{C H}), 60.0\left(\mathrm{C}(\mathrm{O}) \mathrm{NCH}_{2}\right), 47.1\left(\mathrm{NHCH}_{2} \mathrm{CH}_{2}\right), 40.4\left(\mathrm{NHCH}_{2} \mathbf{C H}_{2}\right), 35.5$ $\left(\mathrm{C}(\mathrm{O}) \mathrm{NCH}_{2} \mathrm{CH}_{2} \mathbf{C H}_{2}\right), 28.4\left(\mathrm{C}\left(\mathrm{CH}_{3}\right)_{3}\right), 24.4\left(\mathrm{C}(\mathrm{O}) \mathrm{NCH}_{2} \mathbf{C H}_{2} \mathrm{CH}_{2}\right)$.

\section{tert-butyl 2-((2-(3',4'-dichloro-[1,1'-biphenyl]-4-yl)ethyl)carbamoyl)pyrrolidine-1-} carboxylate (23b)

Using Method J, (tert-butoxycarbon yl)proline ( $0.80 \mathrm{~g}, 3.72 \mathrm{mmol}), N$-methylmorpholine $(1.14$ $\mathrm{mL}, 10.4 \mathrm{mmol})$, isobutyl chloroformate $(0.63 \mathrm{~mL}, 4.83 \mathrm{mmol})$ and $\mathbf{8 e}(1.18 \mathrm{~g}, 3.90 \mathrm{mmol})$ gave 23b as a white solid $(1.07 \mathrm{~g}, 62 \%) ; R_{f}=0.21$ ( $n$-hexane 1: EtOAc 1$) ;{ }^{1} \mathrm{H} \mathrm{NMR}(400 \mathrm{MHz}$, $\left.\mathrm{CDCl}_{3}\right) \delta 7.67(\mathrm{~d}, J=1.8 \mathrm{~Hz}, 1 \mathrm{ArH}), 7.49-7.53(\mathrm{~m}, 3 \mathrm{ArH}), 7.71(\mathrm{dd}, J=1.7 \mathrm{~Hz}, 8.3 \mathrm{~Hz}, 1$ ArH), 7.30 (d, $J=11.0 \mathrm{~Hz}, 2$ ArH), 6.12-6.98 (m, C(O)NH), 4.27 (br s, chiral-H), 3.56 (q, $J$ $=6.2 \mathrm{~Hz}, 6.7 \mathrm{~Hz}, \mathrm{NHCH}_{2} \mathrm{CH}_{2}$ ), 3.36 (br s, 2 pyrrolidine-H), 2.82-2.94 (m, $\mathrm{NHCH}_{2} \mathrm{CH}_{2}$ ), $1.87-$ 2.38 (m, 4 pyrrolidine-H), $1.44\left(\mathrm{~s}, \mathrm{C}\left(\mathrm{CH}_{3}\right)_{3}\right)$.

\subsection{Synthesis of 24}

tert-butyl 3-((2-(4'-(trifluoromethyl)-[1,1'-biphenyl]-4-yl)ethyl)carbamoyl)pyrrolidine-1carboxylate (24a)

Using Method K, to a solution of 1-(tert-butoxycarbonyl)py rrolidine-3-carboxylic acid (0.80 g, $3.72 \mathrm{mmol})$, NMM (0.82 mL. $7.43 \mathrm{mmol})$, IBCF (0.63 mL, $4.83 \mathrm{mmol}), 8 \mathrm{a}$ (1.18 g, 3.90 $\mathrm{mmol})$ in THF anhydrous $(37 \mathrm{~mL})$ gave 24 as a white solid $(1.48 \mathrm{~g}, 85 \%) ; R_{f}=0.51$ (EtOAc 3 : n-hexane 1); ${ }^{1} \mathrm{H}$ NMR (300 MHz, $\left.\mathrm{CDCl}_{3}\right) \delta 7.68$ (s, 4 ArH), 7.57-7.54 (m, 2 ArH), 7.30-7.27 (m, 2 ArH), 5.69 (br s, C(O)NH), 3.59-3.25 (m, C(O)NCH $\left.\mathbf{H}_{2} \mathrm{CH}, \mathrm{NHCH}_{2} \mathrm{CH}_{2}\right), 2.91-2.77$ (m, $\left.\mathrm{CH}_{2} \mathrm{CH}_{2} \mathrm{CH}\right), 2.18-2.01\left(\mathrm{~m}, \mathrm{CH}_{2} \mathrm{CH}_{2} \mathrm{CH}\right), 1.45\left(\mathrm{~s}, \mathrm{C}\left(\mathrm{CH}_{3}\right)_{3}\right) ;{ }^{13} \mathrm{C} \mathrm{NMR}\left(75 \mathrm{MHz}, \mathrm{CDCl}_{3}\right) \delta$ $172.2(\mathbf{C}(\mathrm{O}) \mathrm{NH}), 154.4(\mathbf{C}(\mathrm{O}) \mathrm{ONH}), 144.3,138.9,138.1,129.4,129.3\left(\mathrm{q}, J_{\mathrm{C}-\mathrm{F}}=32.1 \mathrm{~Hz}\right)$, 127.5, 127.3, 125.8 (q, $\left.J_{\mathrm{C}-\mathrm{F}}=3.8 \mathrm{~Hz}\right), 124.3\left(\mathrm{q}, J_{\mathrm{C}-\mathrm{F}}=270.2 \mathrm{~Hz}, \mathbf{C F}_{3}\right)(\mathrm{ArC}), 79.5\left(\mathbf{C}\left(\mathrm{CH}_{3}\right)_{3}\right)$, $48.7\left(\mathrm{NCH}_{2} \mathrm{CH}_{2}\right), 45.2\left(\mathrm{CH}_{2} \mathrm{NCH}_{2}\right), 40.6(\mathbf{C H}), 35.3\left(\mathrm{NCH}_{2} \mathrm{CH}_{2}\right), 29.2\left(\mathrm{NCH}_{2} \mathrm{CH}_{2} \mathrm{CH}\right), 28.5$ $\left(\mathrm{C}\left(\mathrm{CH}_{3}\right)_{3}\right)$. 


\subsection{Synthesis of 25}

\section{tert-butyl 2-((2-(3',4'-dichloro-[1,1'-biphenyl]-4-yl)ethyl)carbamoyl)piperidine-1-}

carboxylate (25b)

Using Method F, 1-(tert-butoxycarbon yl)piperidine-2-carboxylic acid (1.00 g, $4.36 \mathrm{mmol}), \mathrm{N}$ methylmorpholine $(1.34 \mathrm{~mL}, 12.2 \mathrm{mmol})$, isobutyl chloroformate $(0.74 \mathrm{~mL}, 5.67 \mathrm{mmol})$ and 8e (1.39 g, $4.58 \mathrm{mmol})$ gave $\mathbf{2 5 b}$ as a white solid (1.28 g, 61\%); $R_{f}=0.70$ ( $n$-hexane 3: EtOAc 1); ${ }^{1} \mathrm{H}$ NMR (400 MHz, $\left.\mathrm{CDCl}_{3}\right) \delta 7.67(\mathrm{~d}, J=2.0 \mathrm{~Hz}, 1 \mathrm{ArH}), 7.50-7.53$ (m, $\left.3 \mathrm{ArH}\right), 7.71$ (dd, $J=2.0 \mathrm{~Hz}, 8.3 \mathrm{~Hz}, 1 \mathrm{ArH}), 7.30$ (d, $J=8.1 \mathrm{~Hz}, 2 \mathrm{ArH}), 6.16$ (br s, C(O)NH), 4.74 (br s, chiralH), 3.99 (br s, 1 piperidine-H), 3.52-3.68 (m, $\left.\mathrm{NHCH}_{2} \mathrm{CH}_{2}\right), 2.84-2.96$ (m, $\left.\mathrm{NHCH}_{2} \mathrm{CH}_{2}\right), 2.63$ (br s, 1 piperidine-H), 2.32 ( $\mathrm{br} \mathrm{s}, 1$ piperidine-H), 1.45-1.68 (m, 5 piperidine-H), 1.45 (s, $\left.\mathrm{C}\left(\mathrm{CH}_{3}\right)_{3}\right)$. 


\section{4. ${ }^{1} \mathrm{H}$ and ${ }^{13} \mathrm{C}$ NMR spectra}
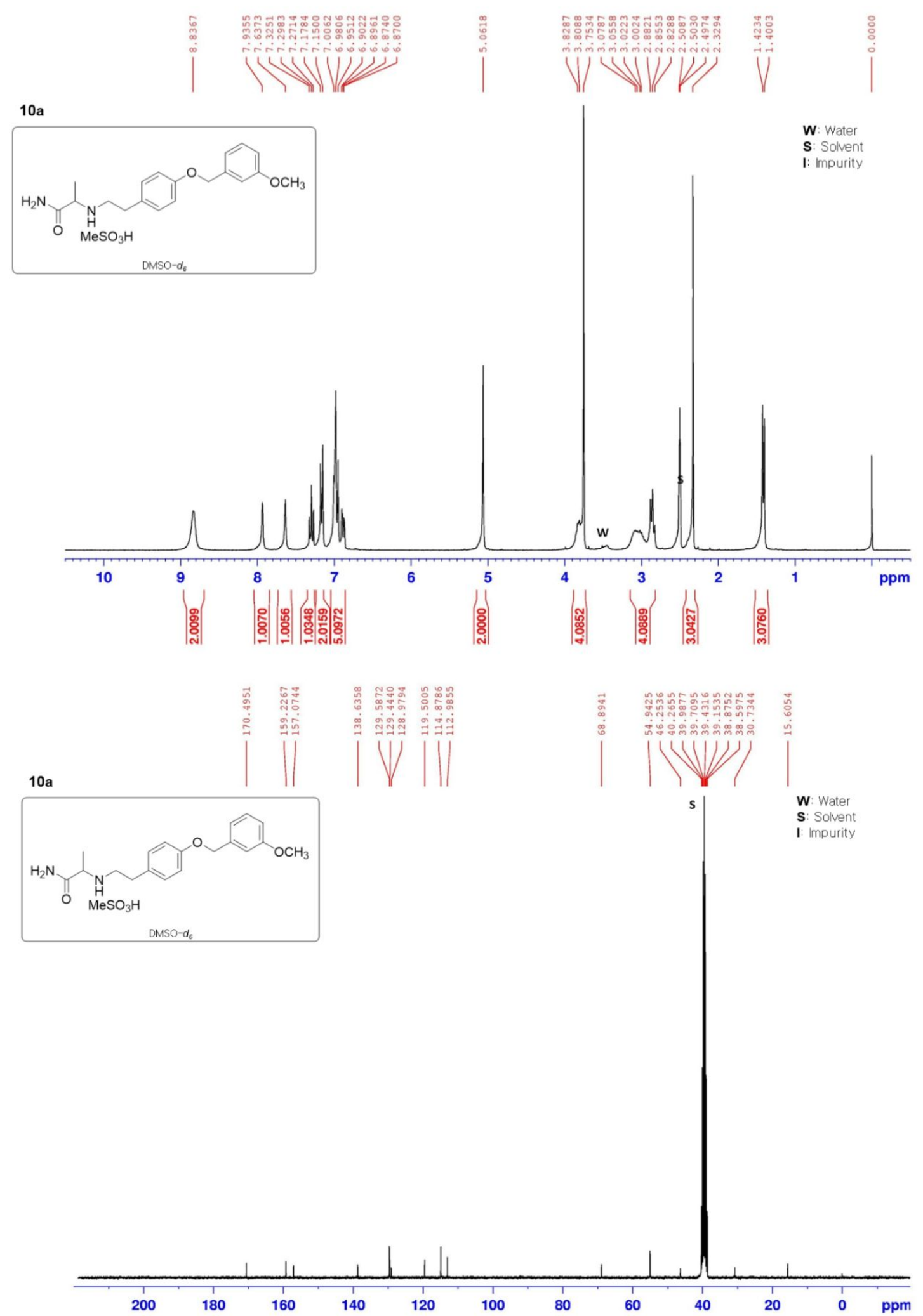

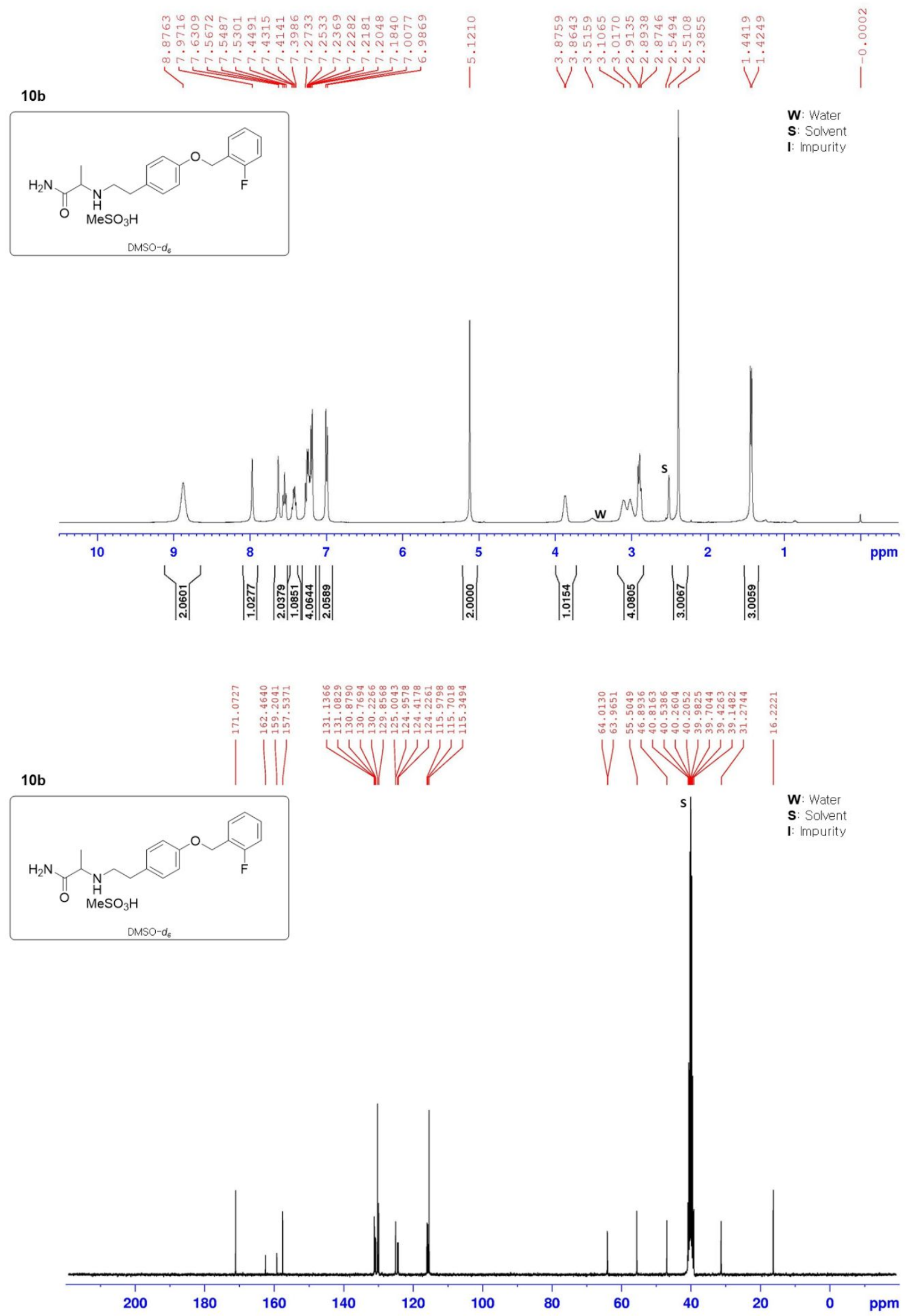

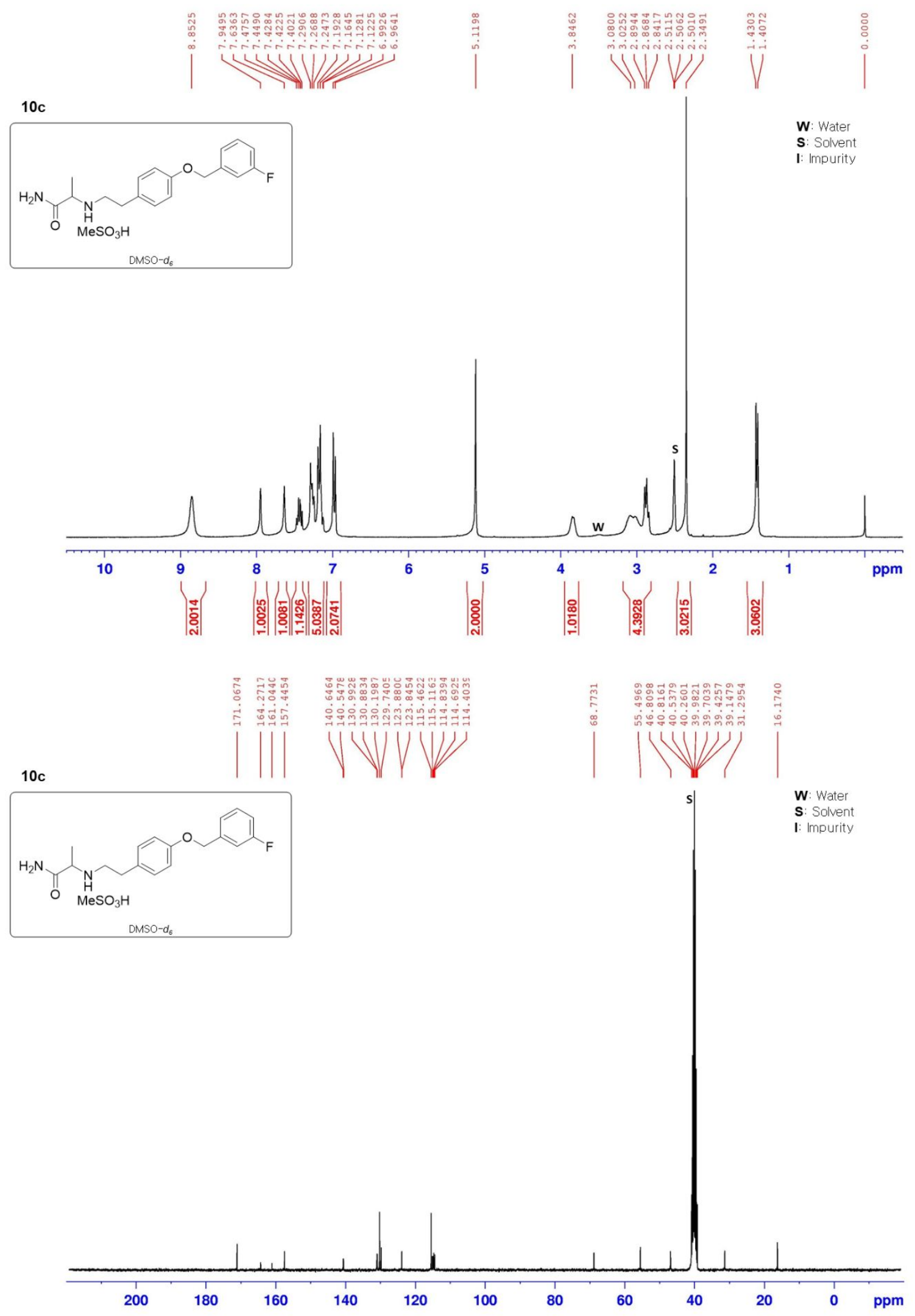

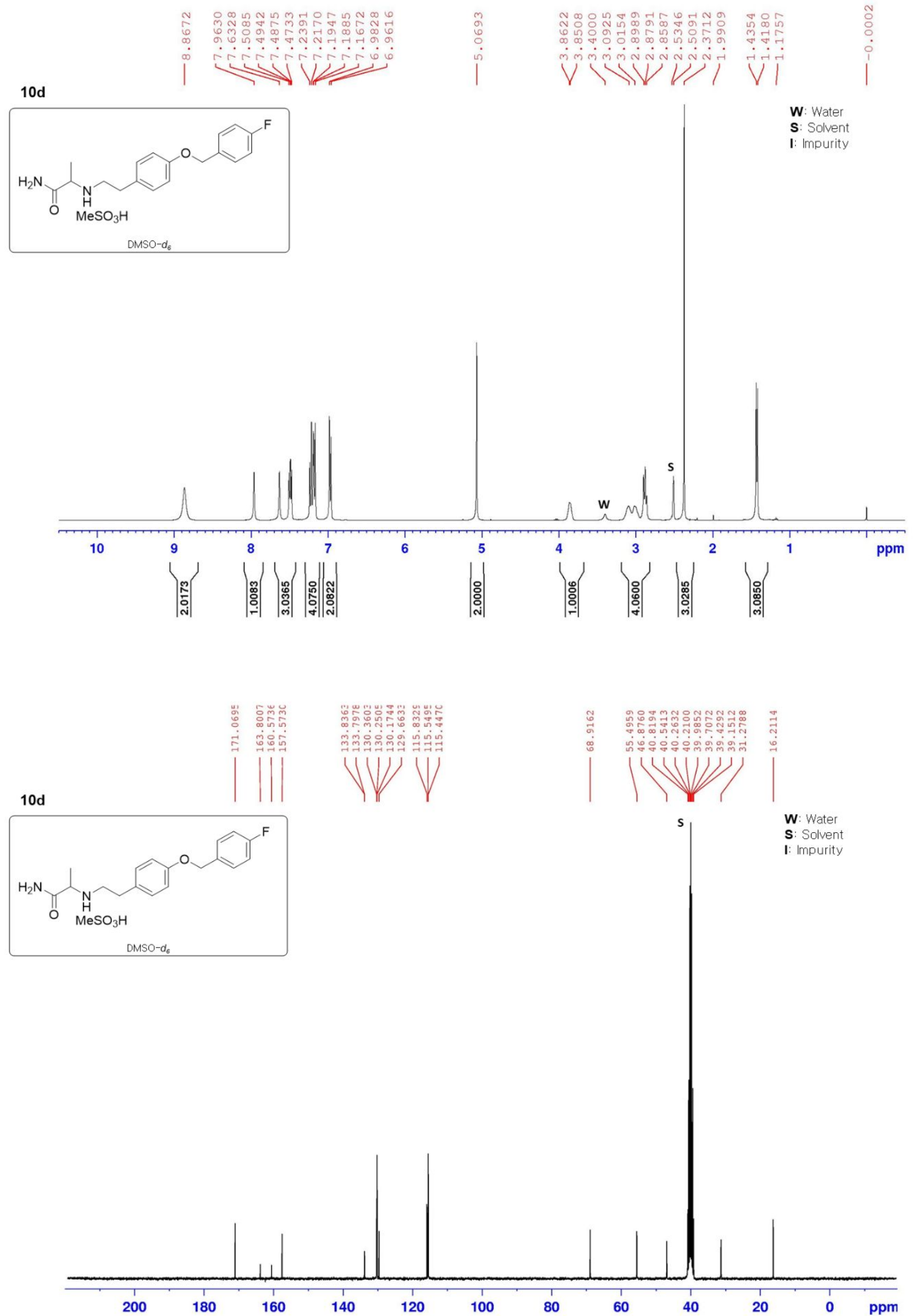
$10 \mathrm{e}$

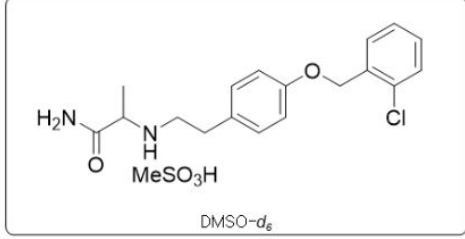

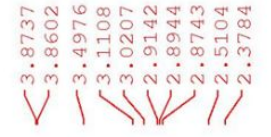

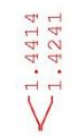

W: Water

S: Solvent

I: Impurity

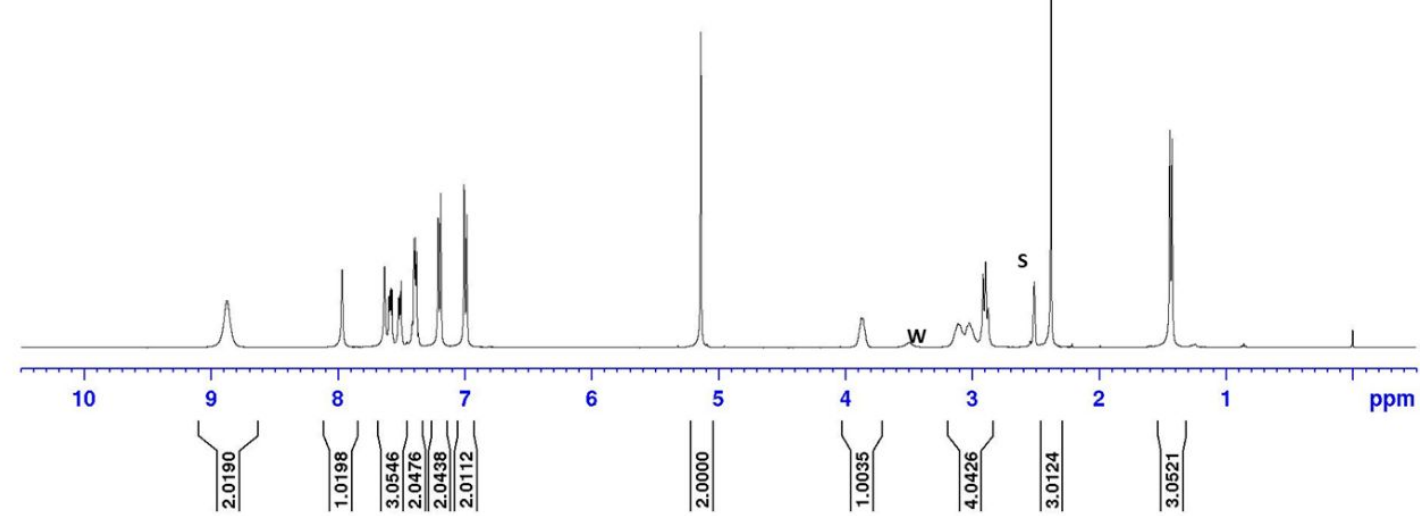

$10 \mathrm{e}$

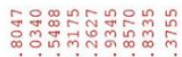

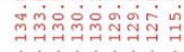

WW

(c)

DMSO-d

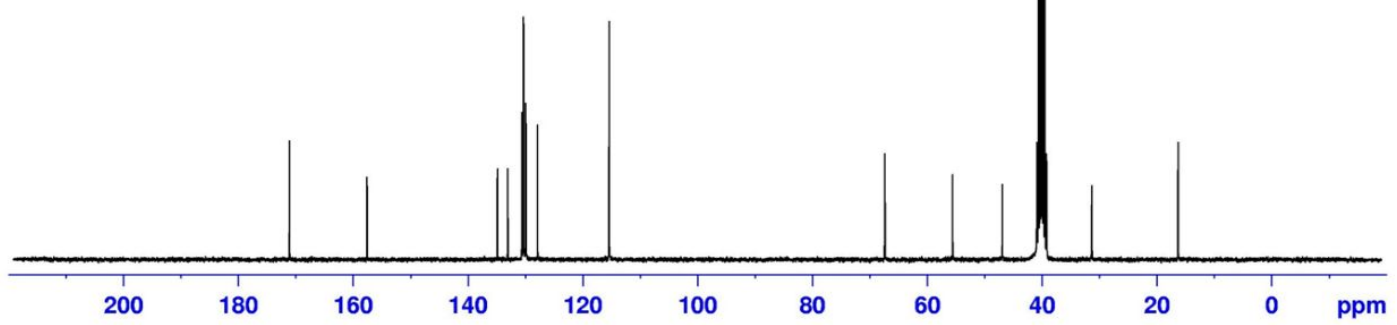


$10 f$

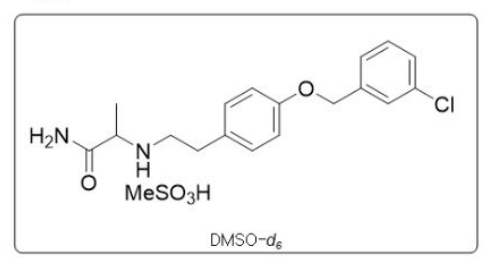

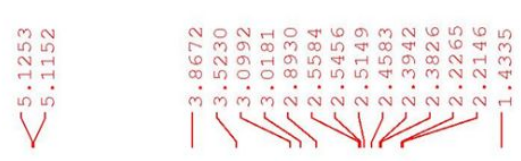

W: Water

S: Solvent

I: Impurity
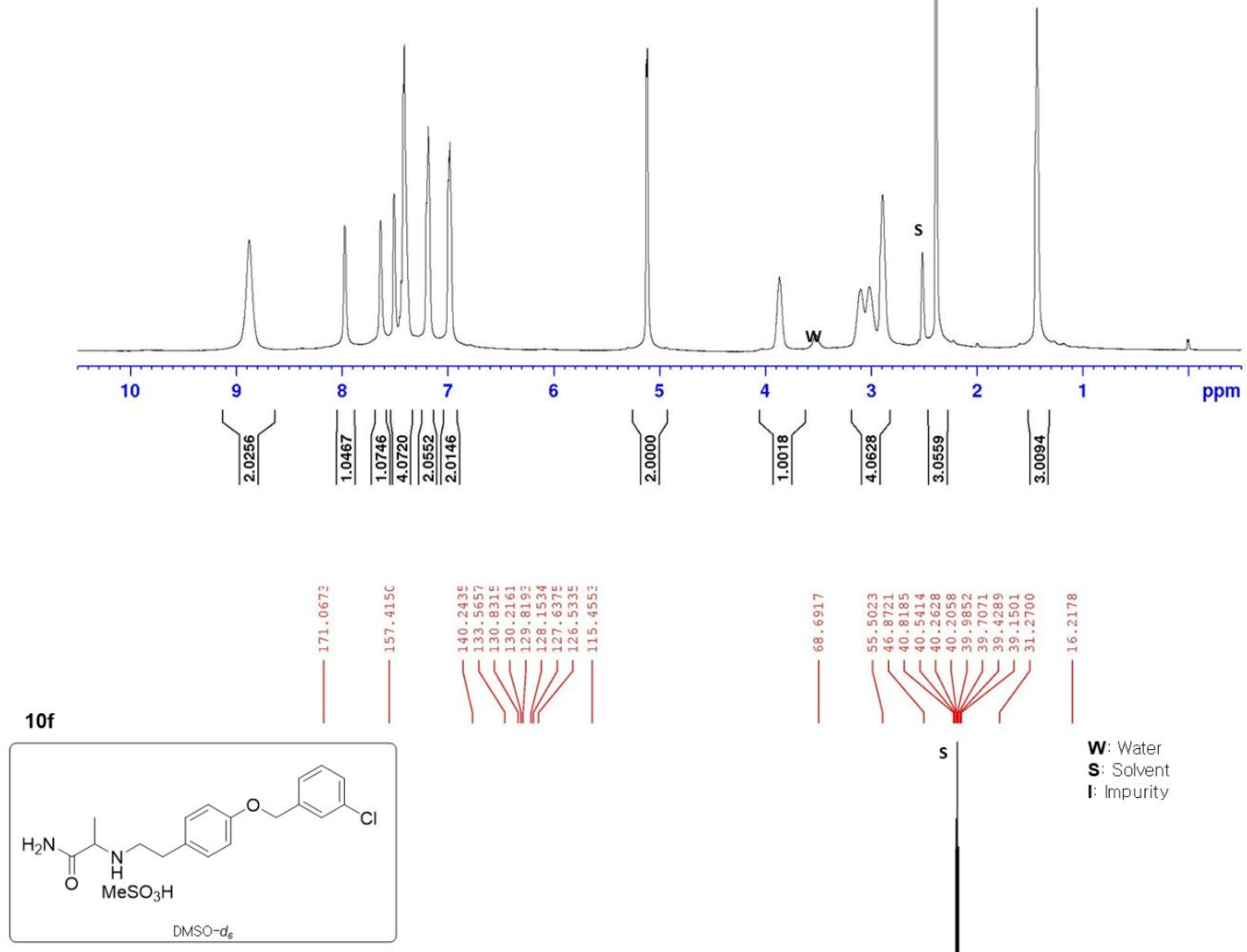

W: Water

S: Solvent I: Impurity

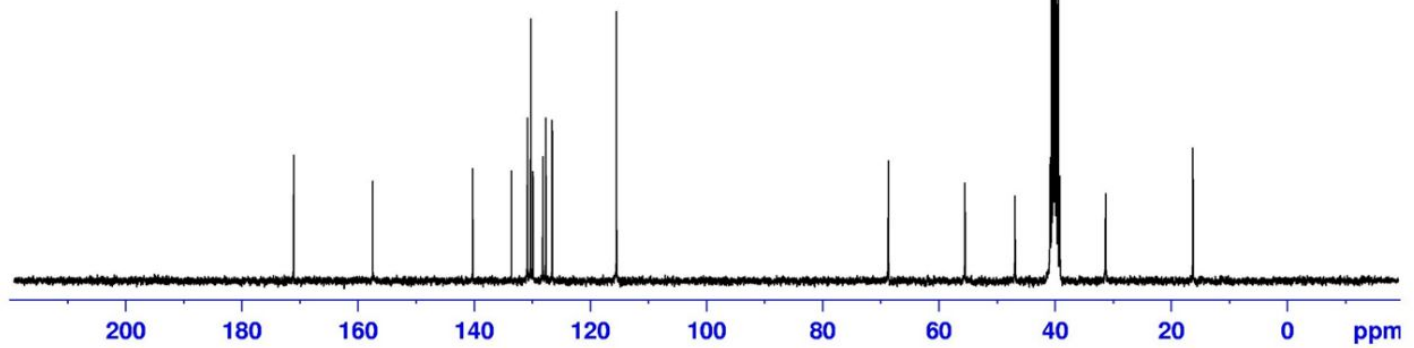



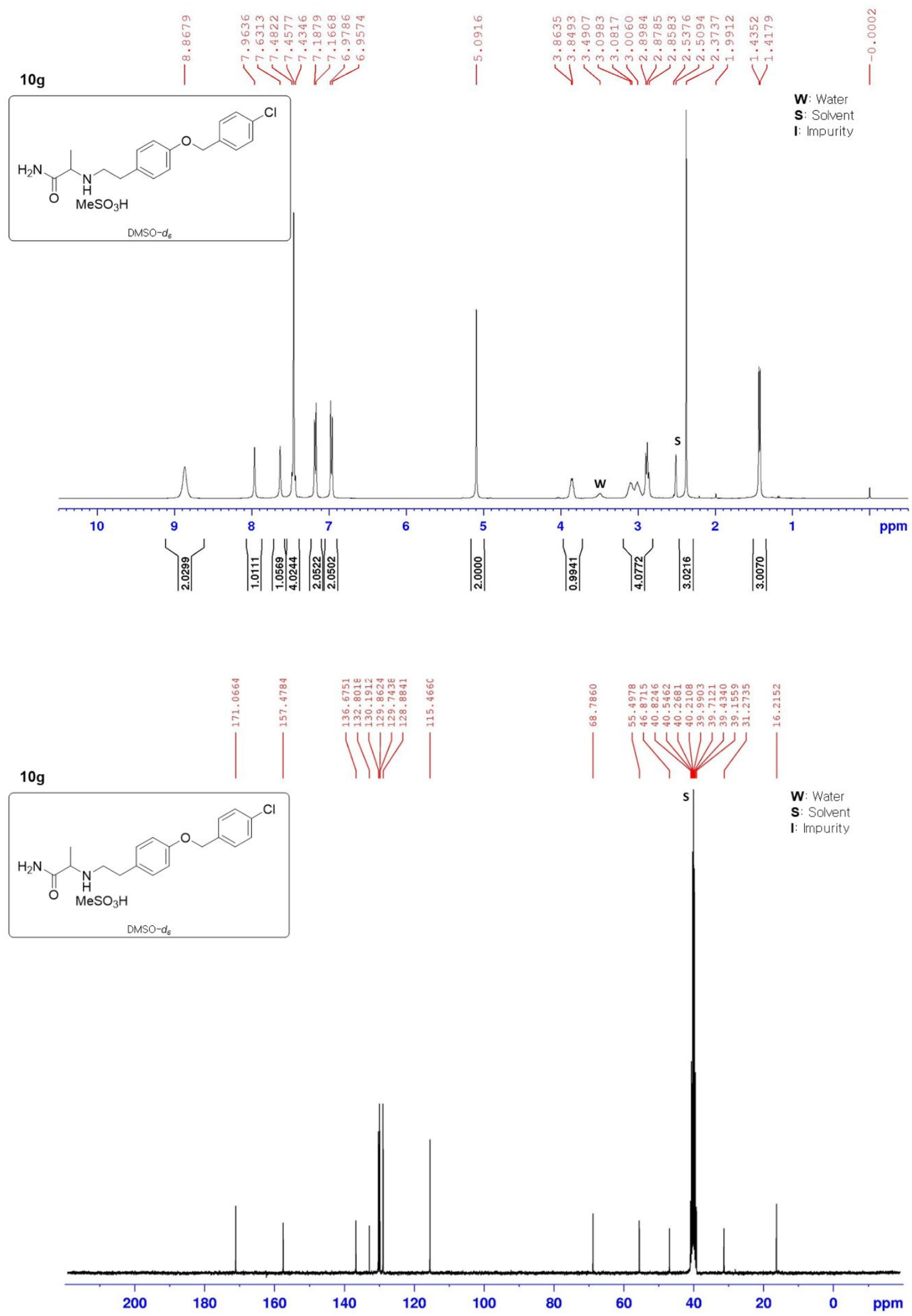

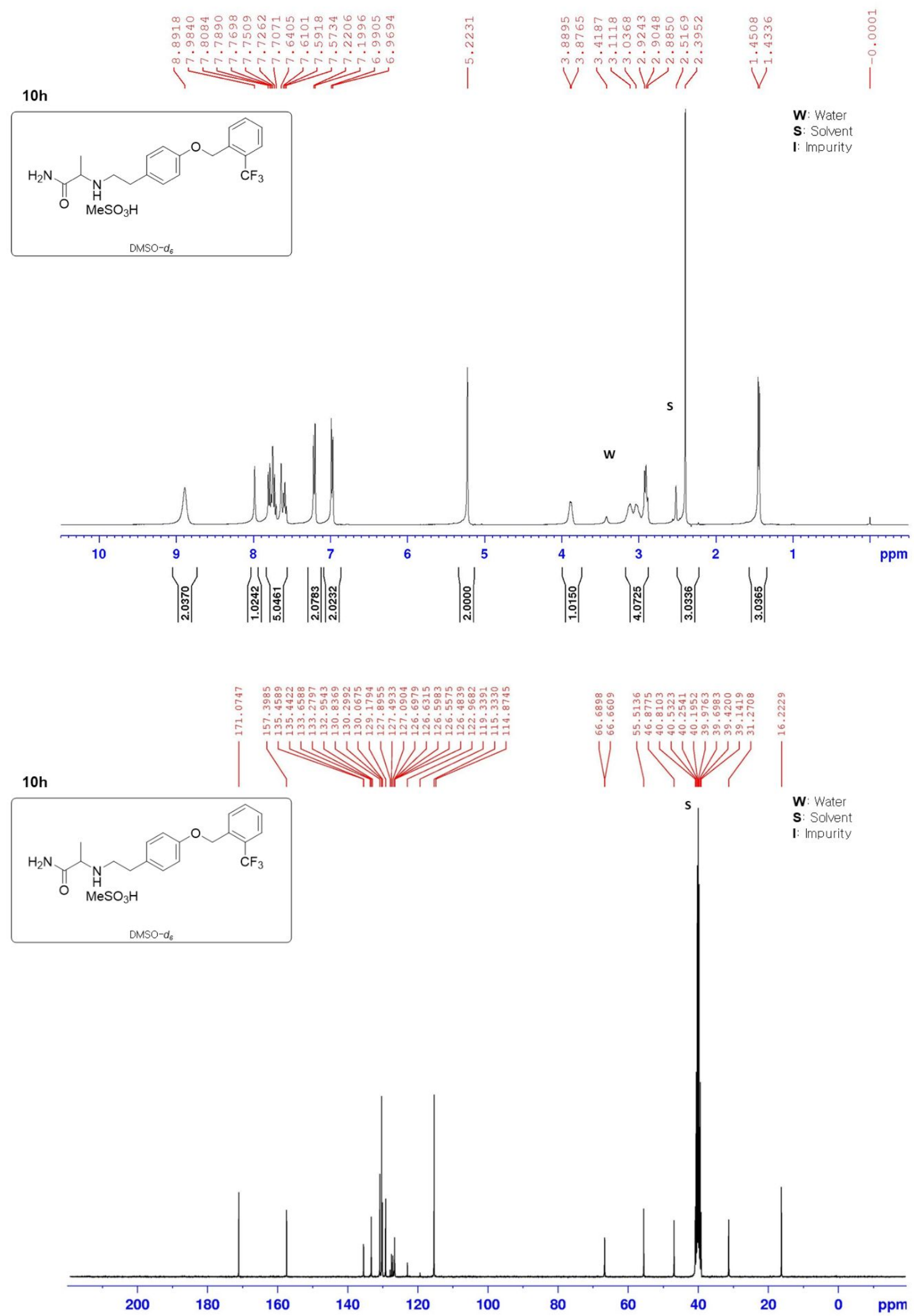

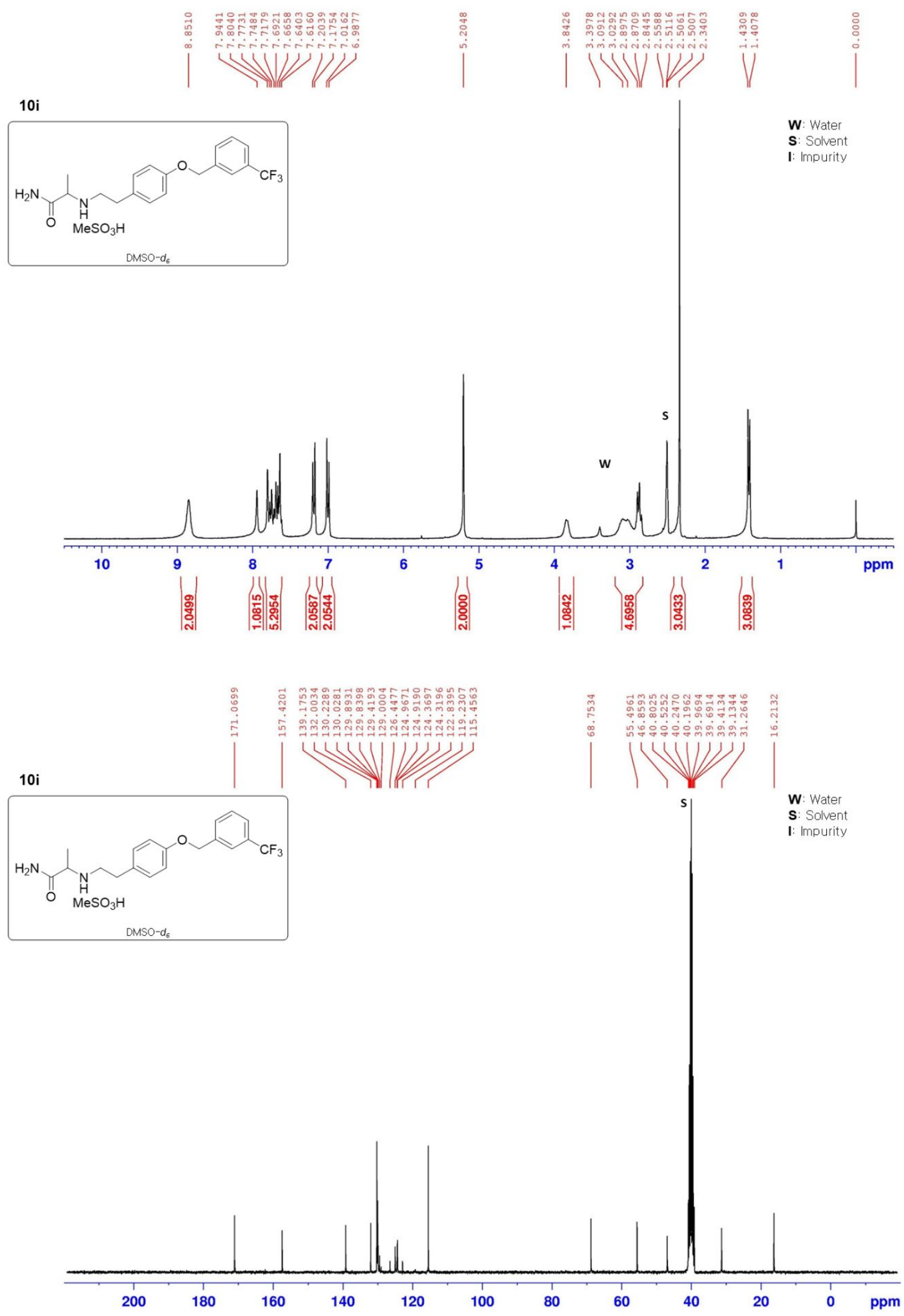

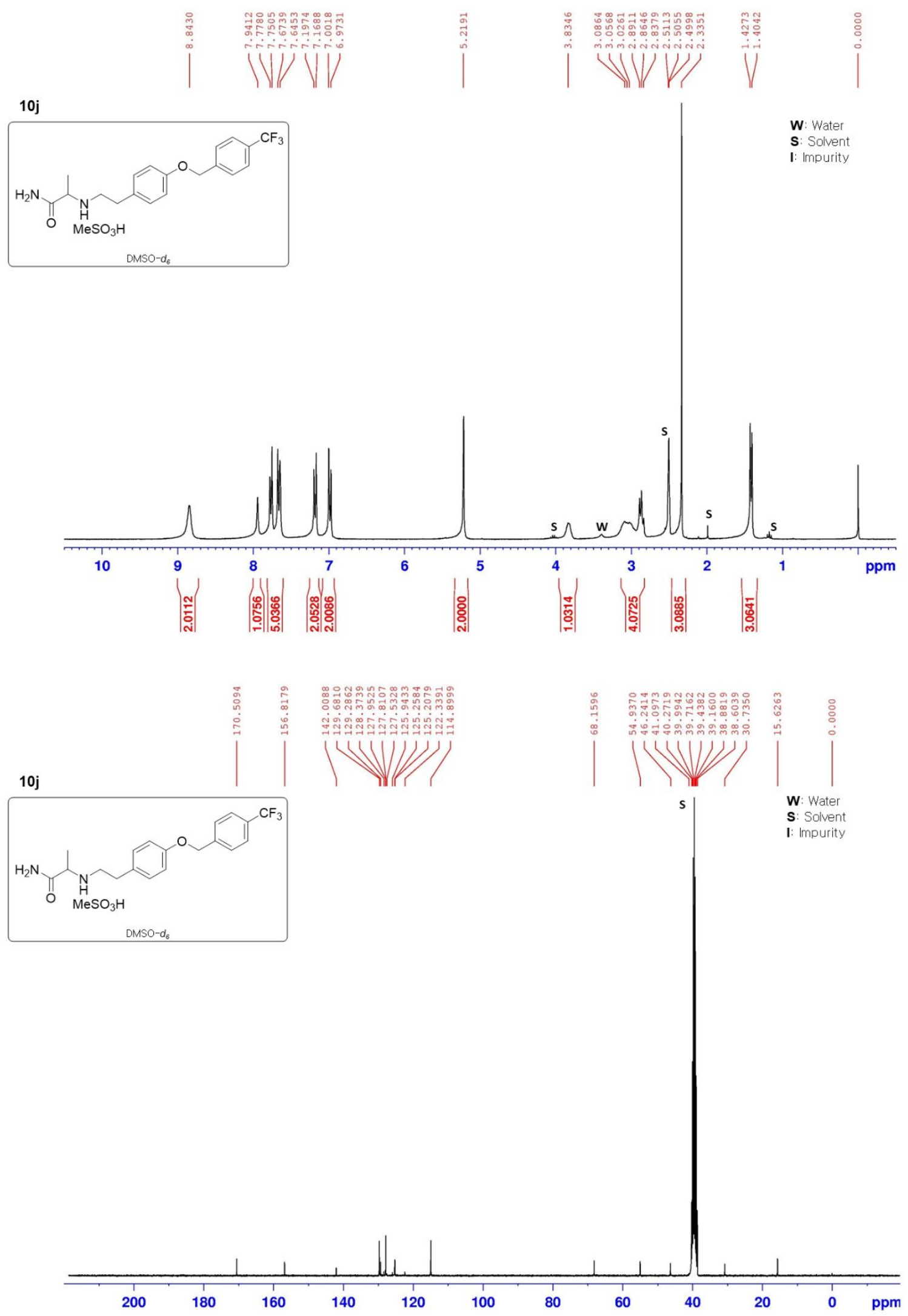

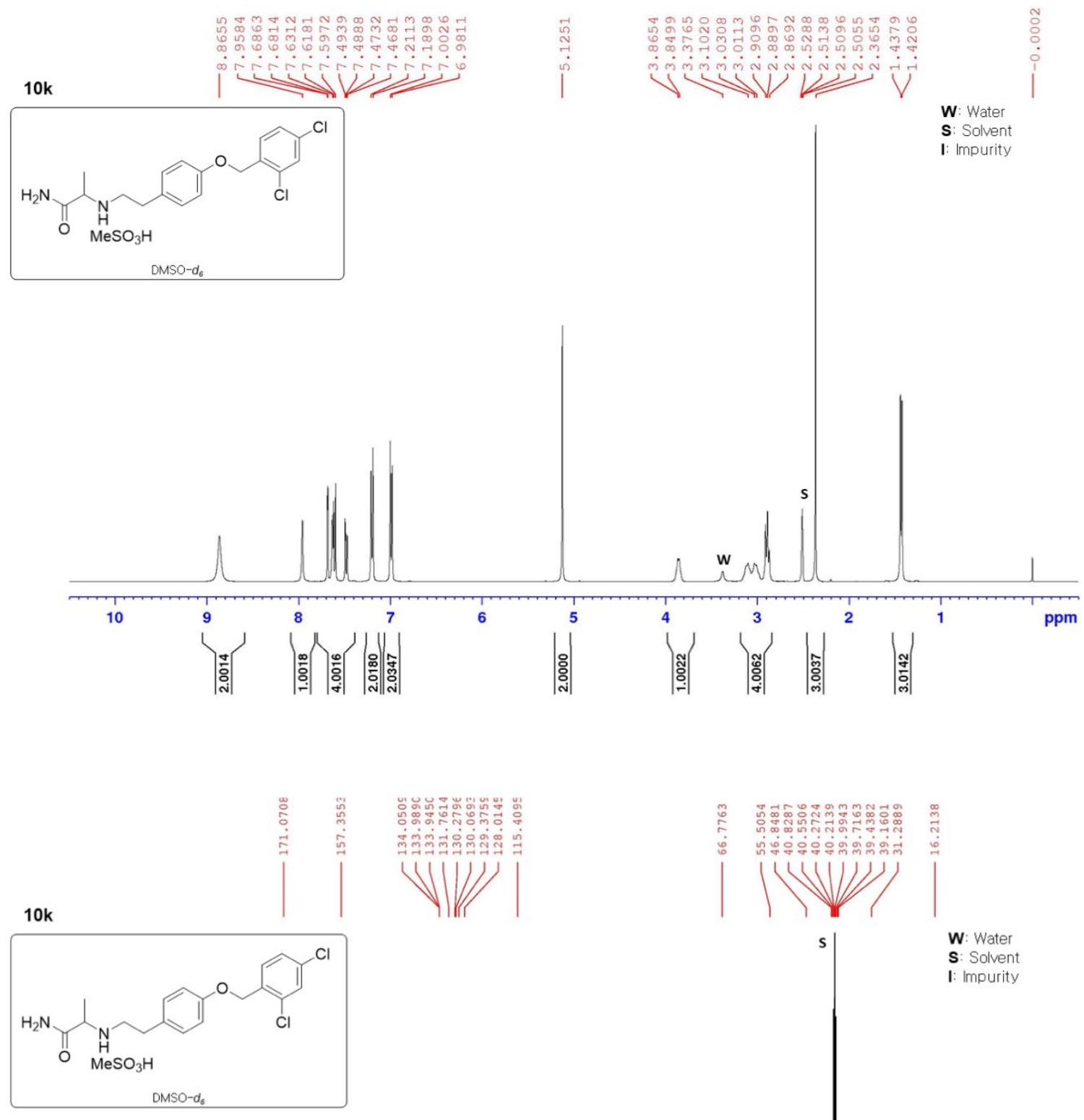

W: Water

S: Solvent

I: Impurity

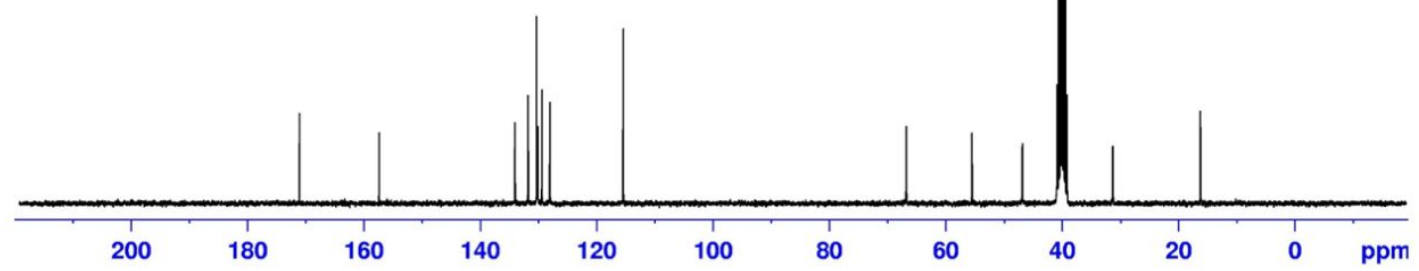



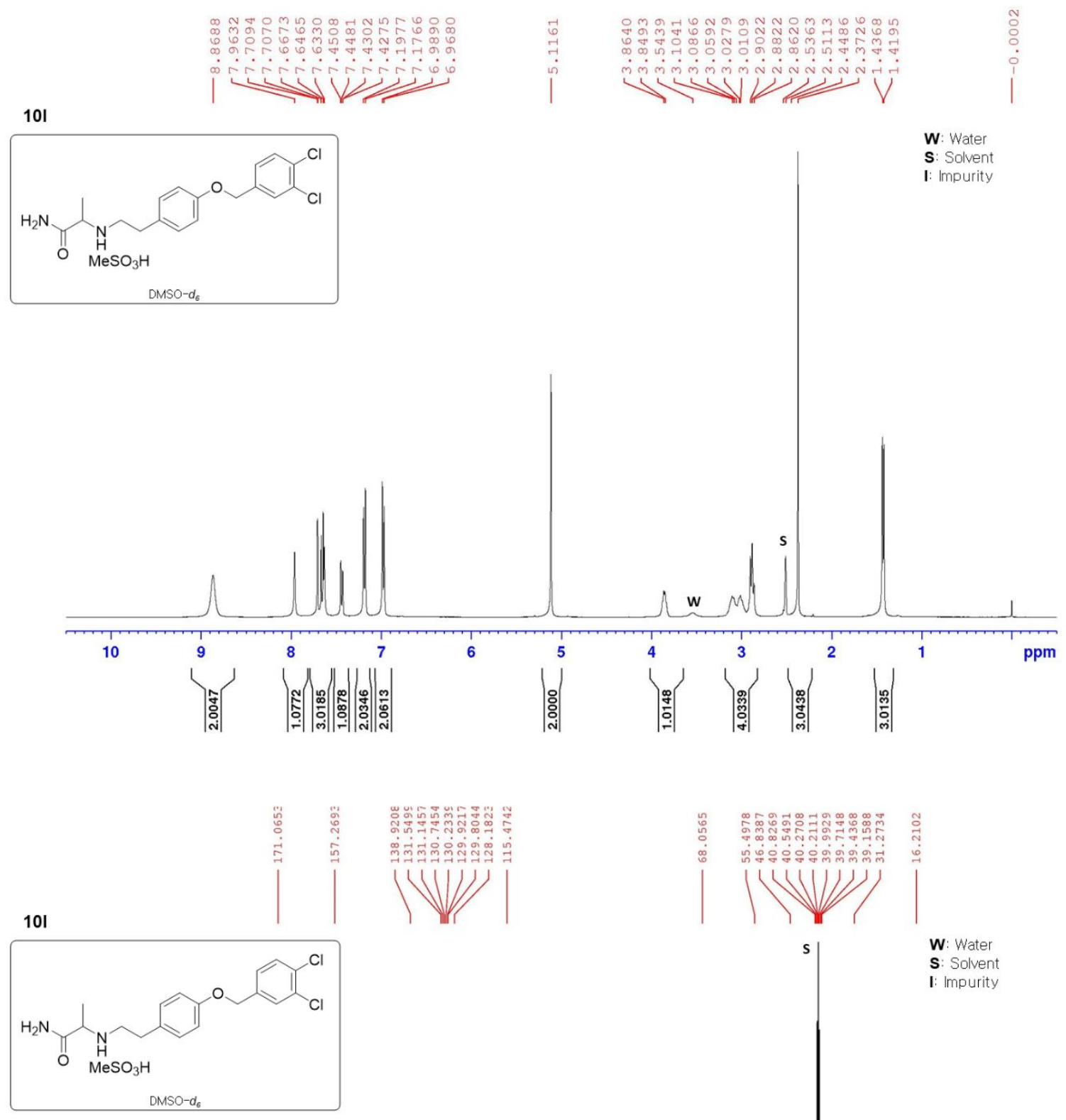

W: Water

S: Solvent I: Impurity

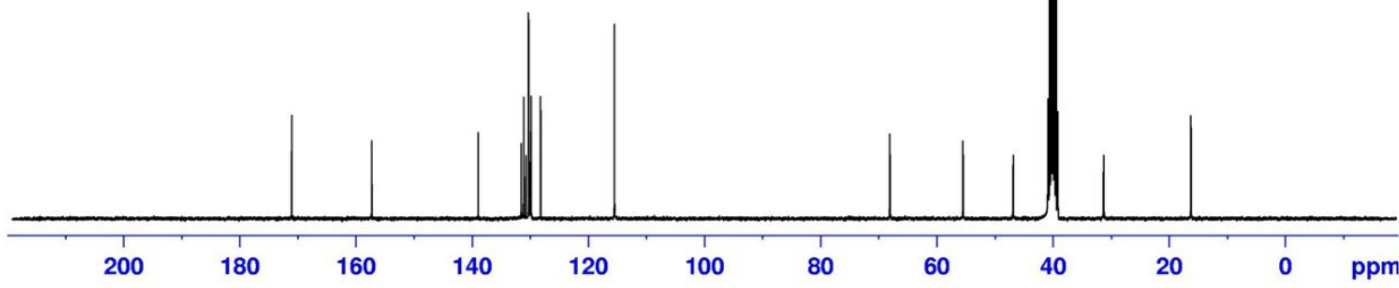




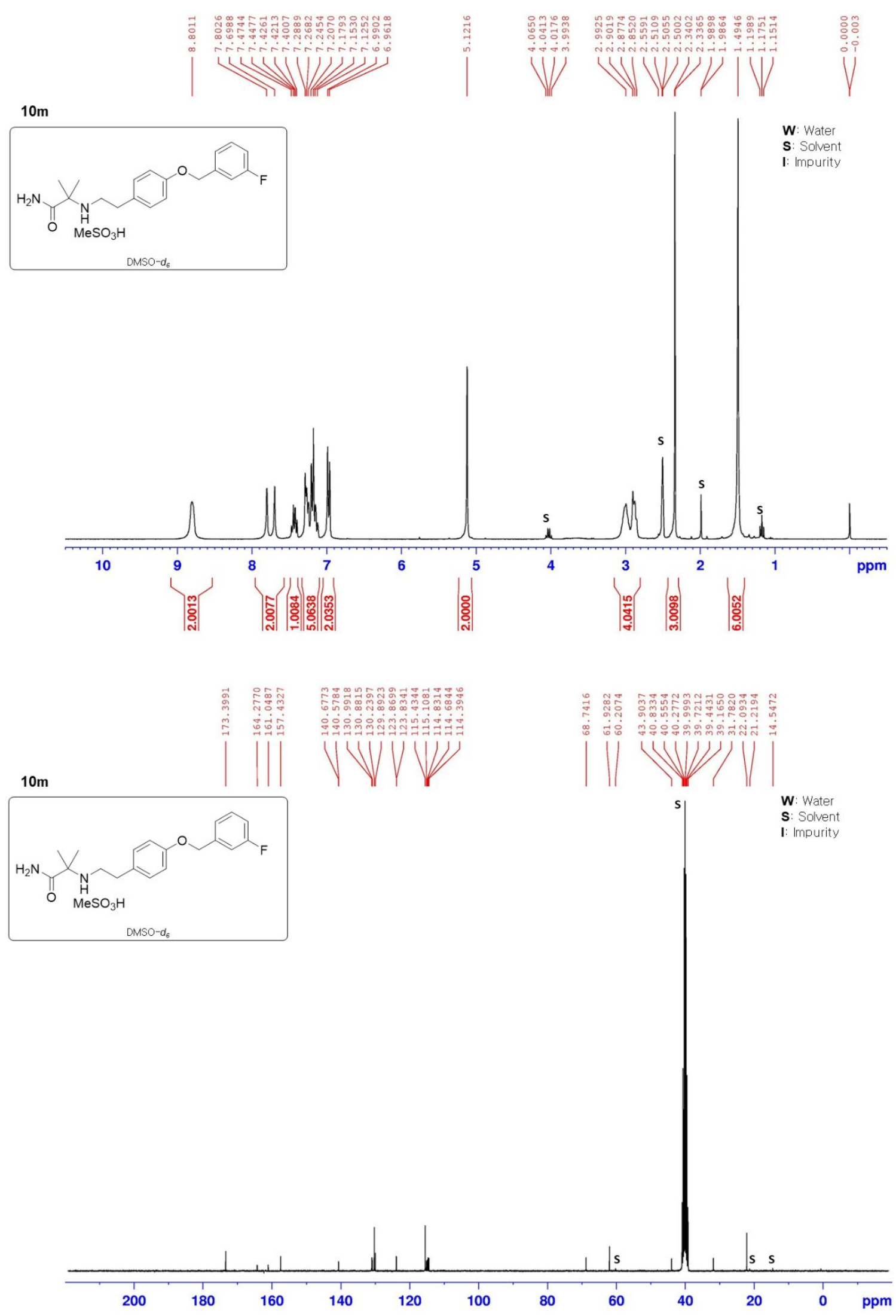



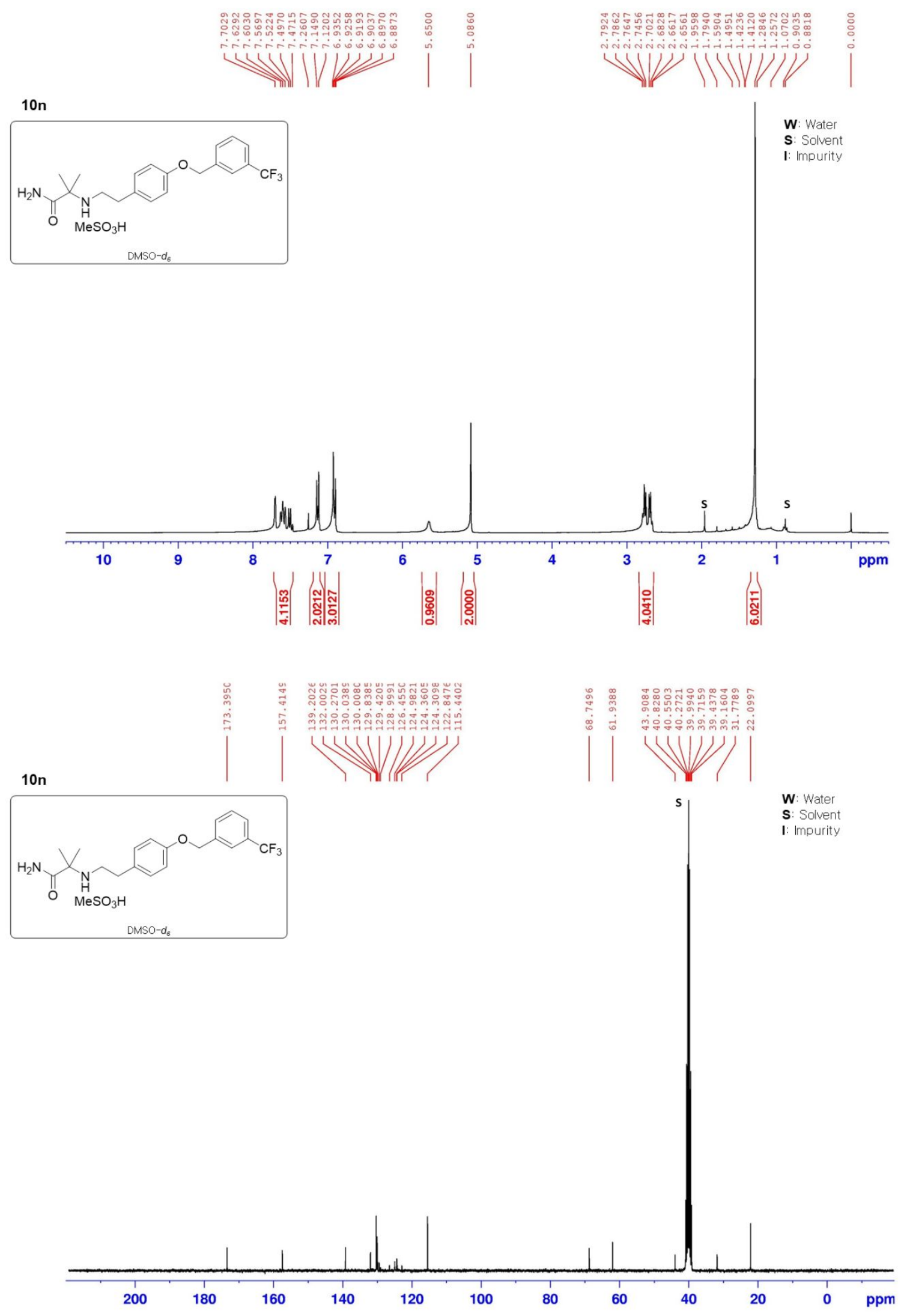

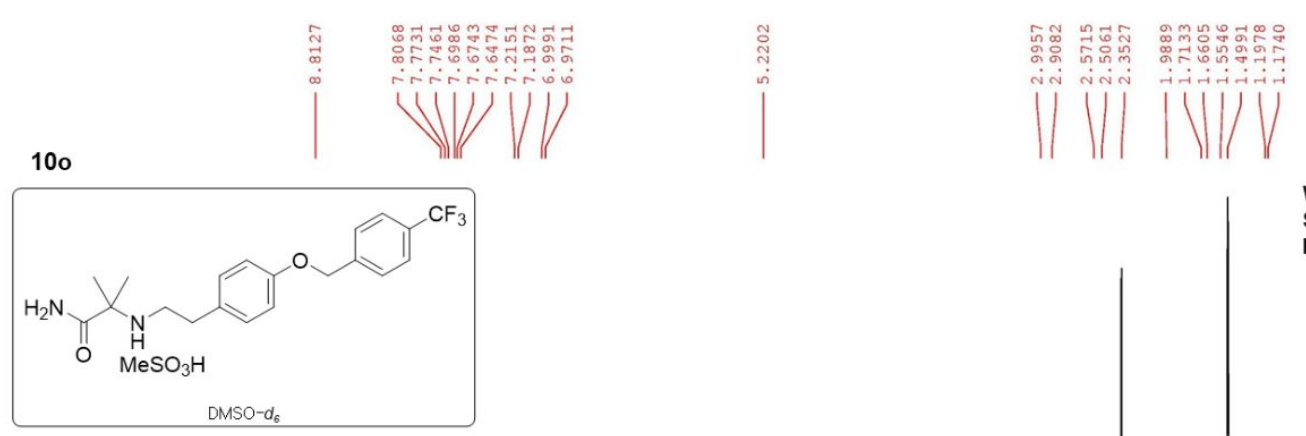

W: Water

S: Solvent

DMSO-d
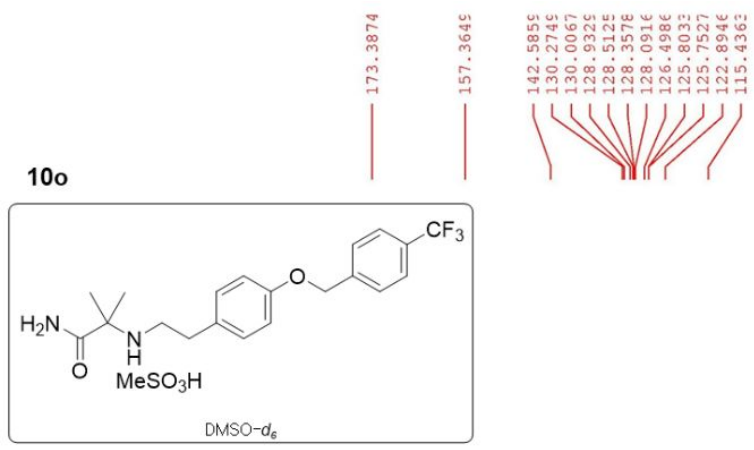

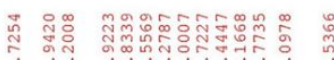

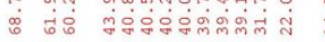

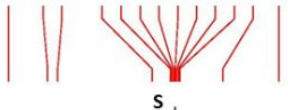

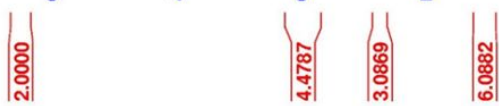

ppm |ల్లో:

$$
\begin{aligned}
& \text { W: Water } \\
& \text { S: Solvent } \\
& \text { I: Impurity }
\end{aligned}
$$

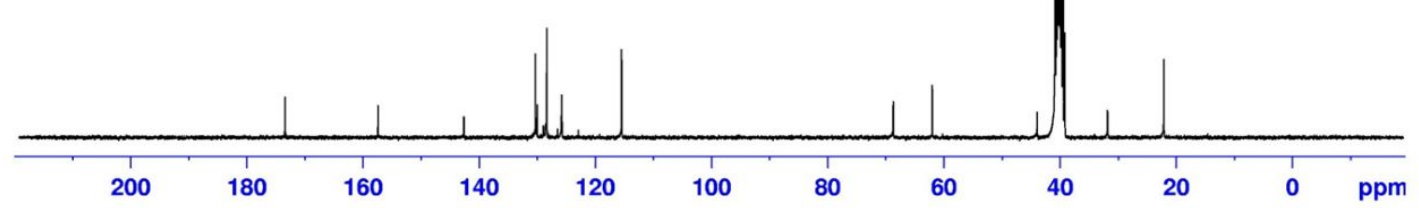



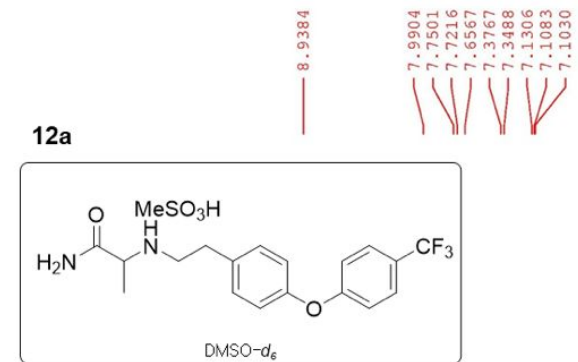

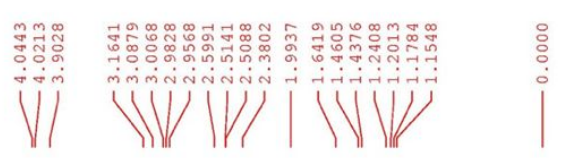

W: Water

S: Solvent I: Impurity
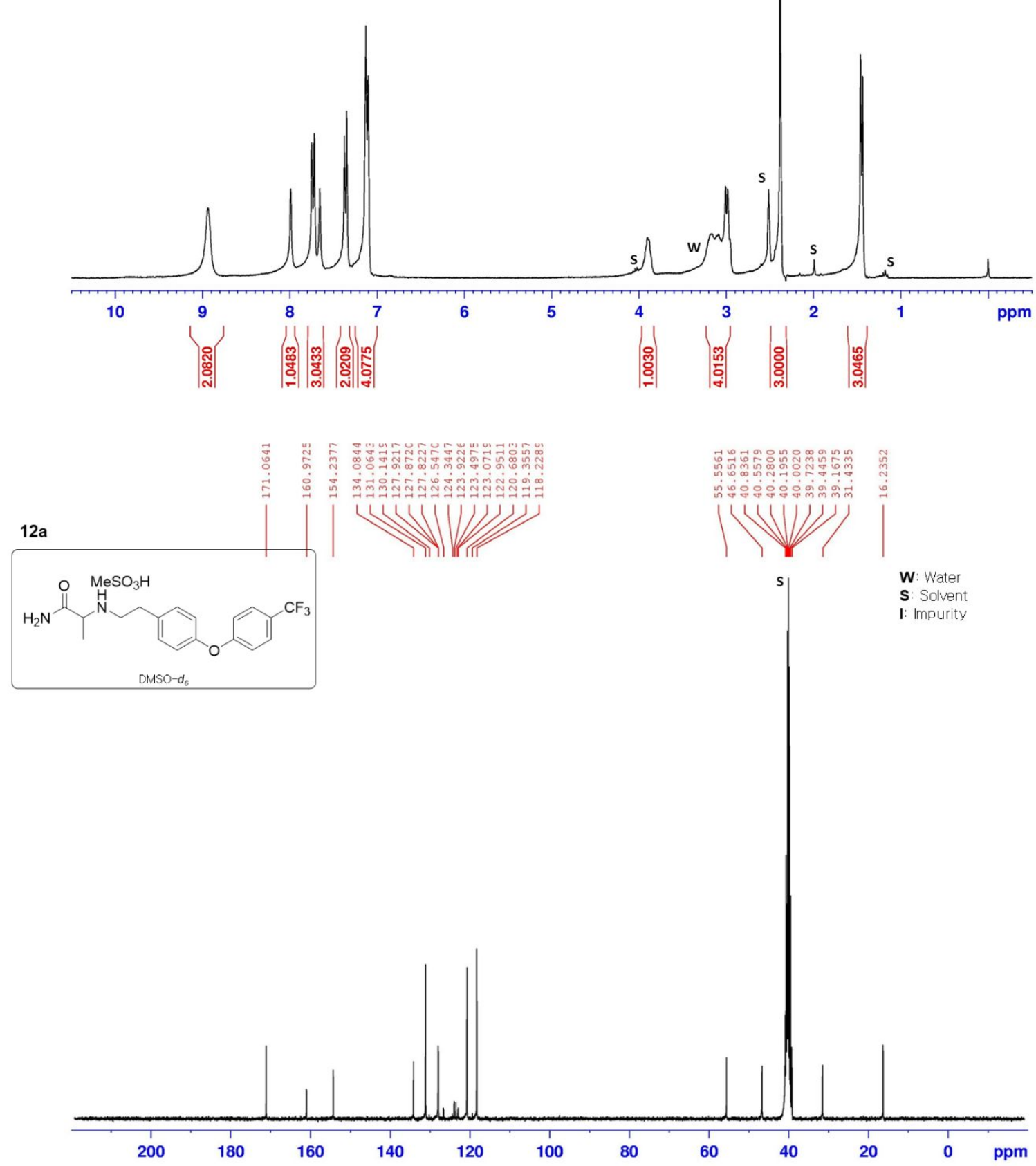

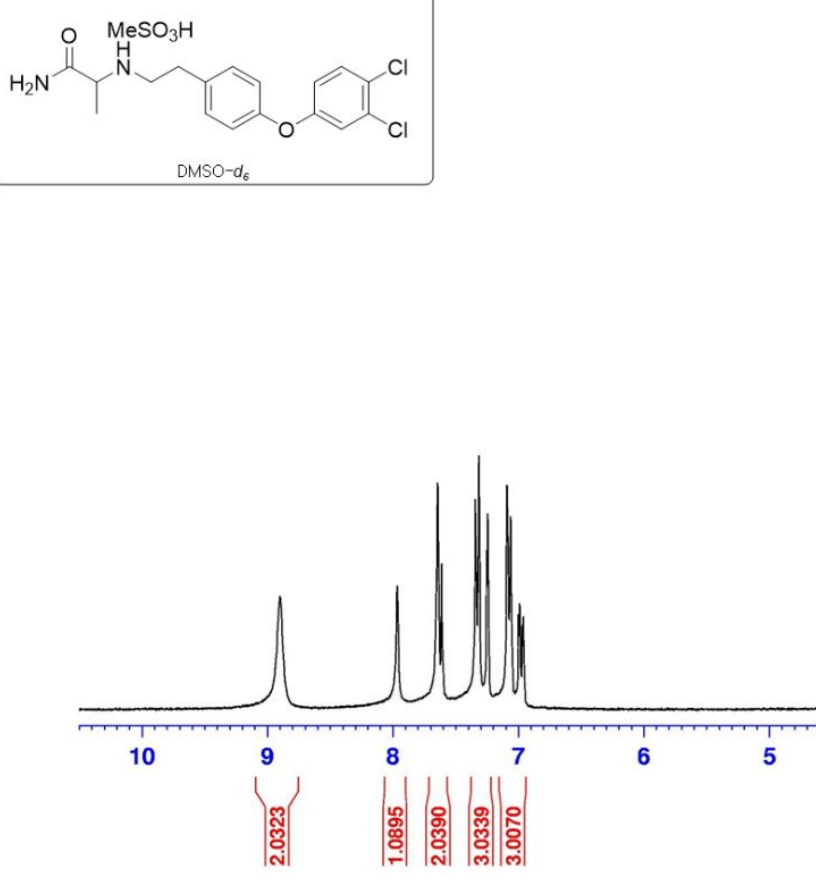

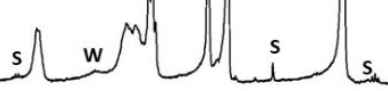

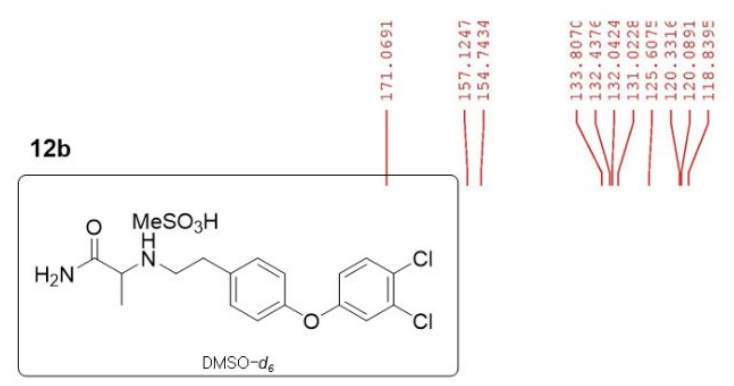

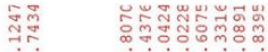
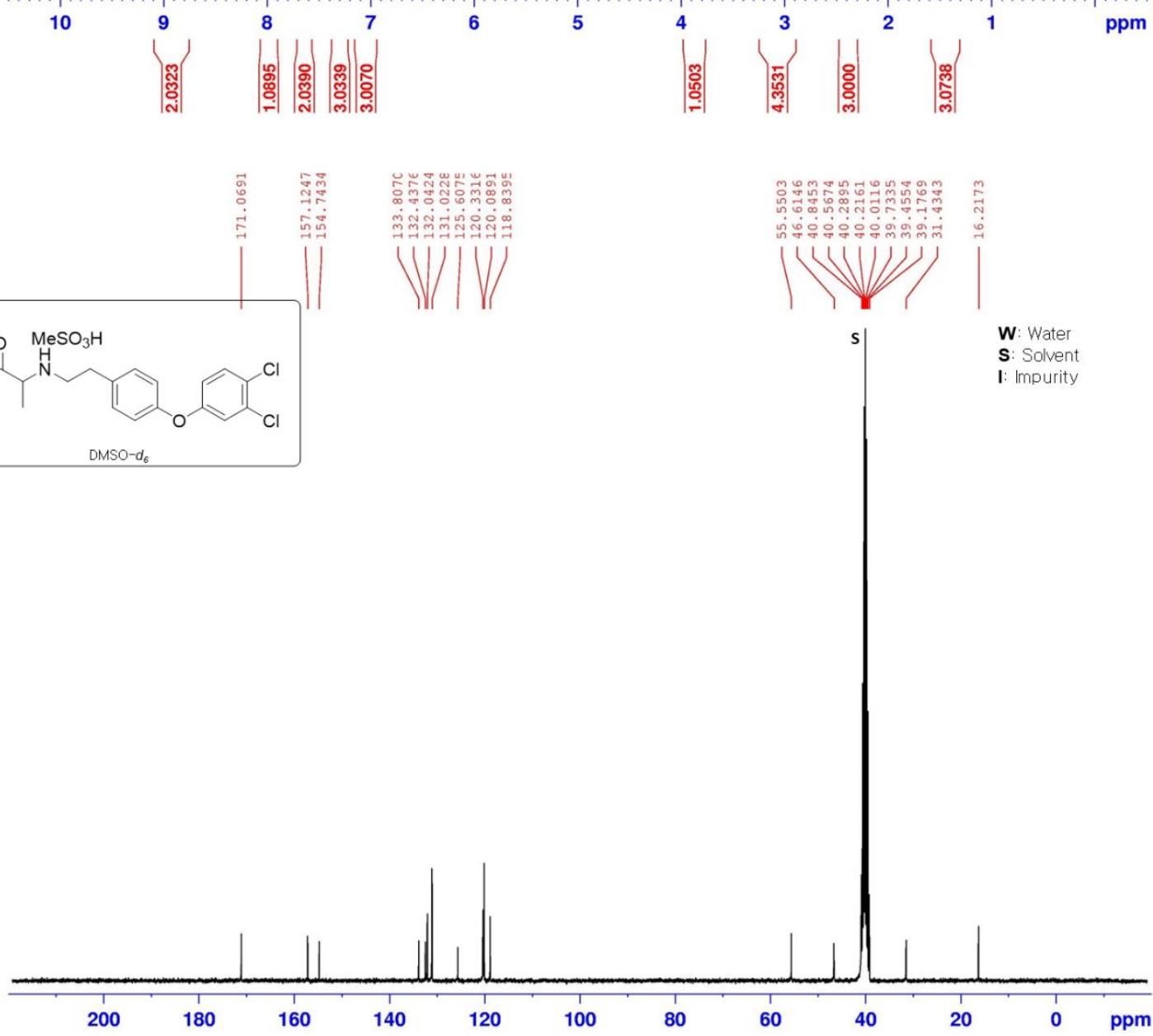

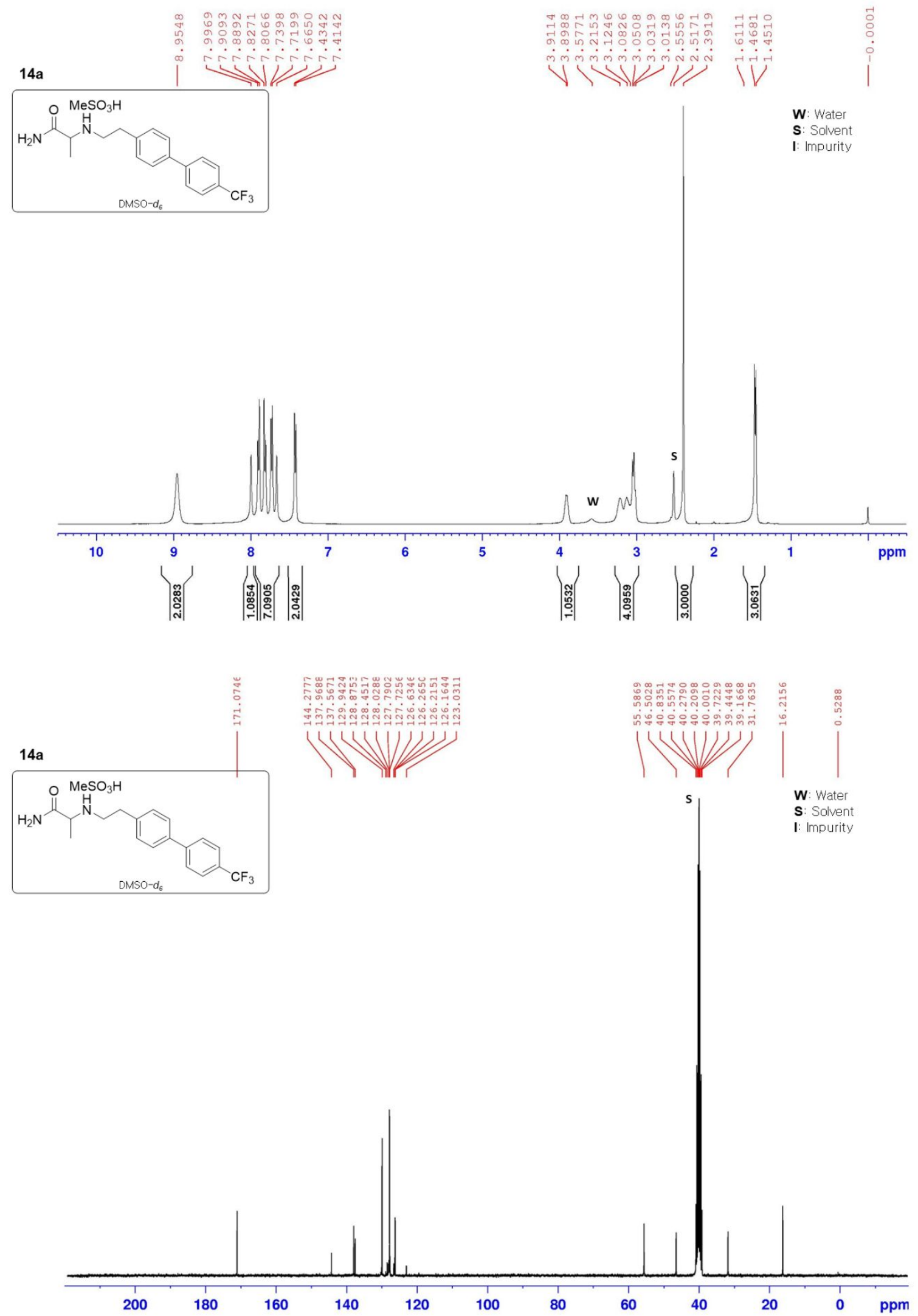

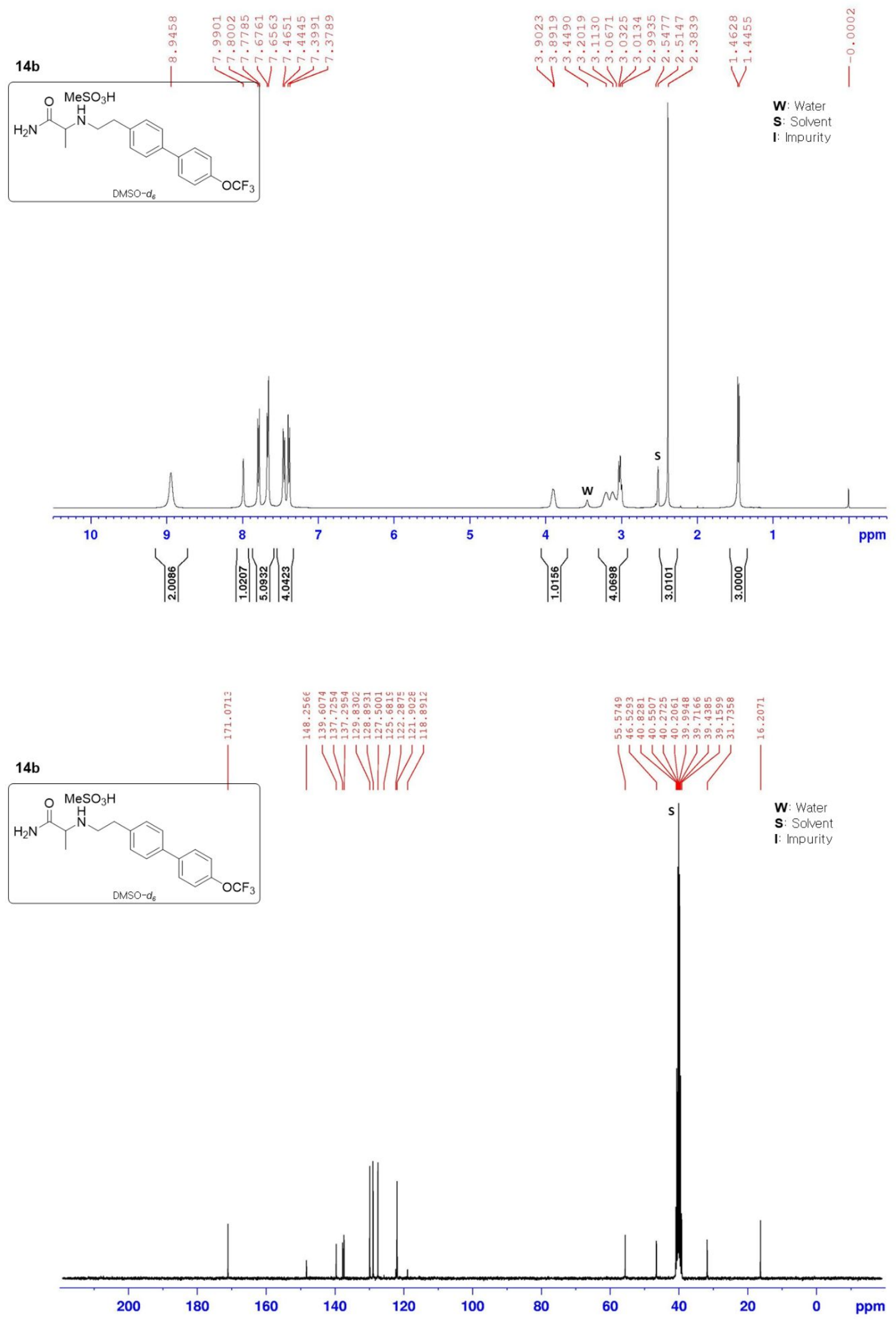

S59 

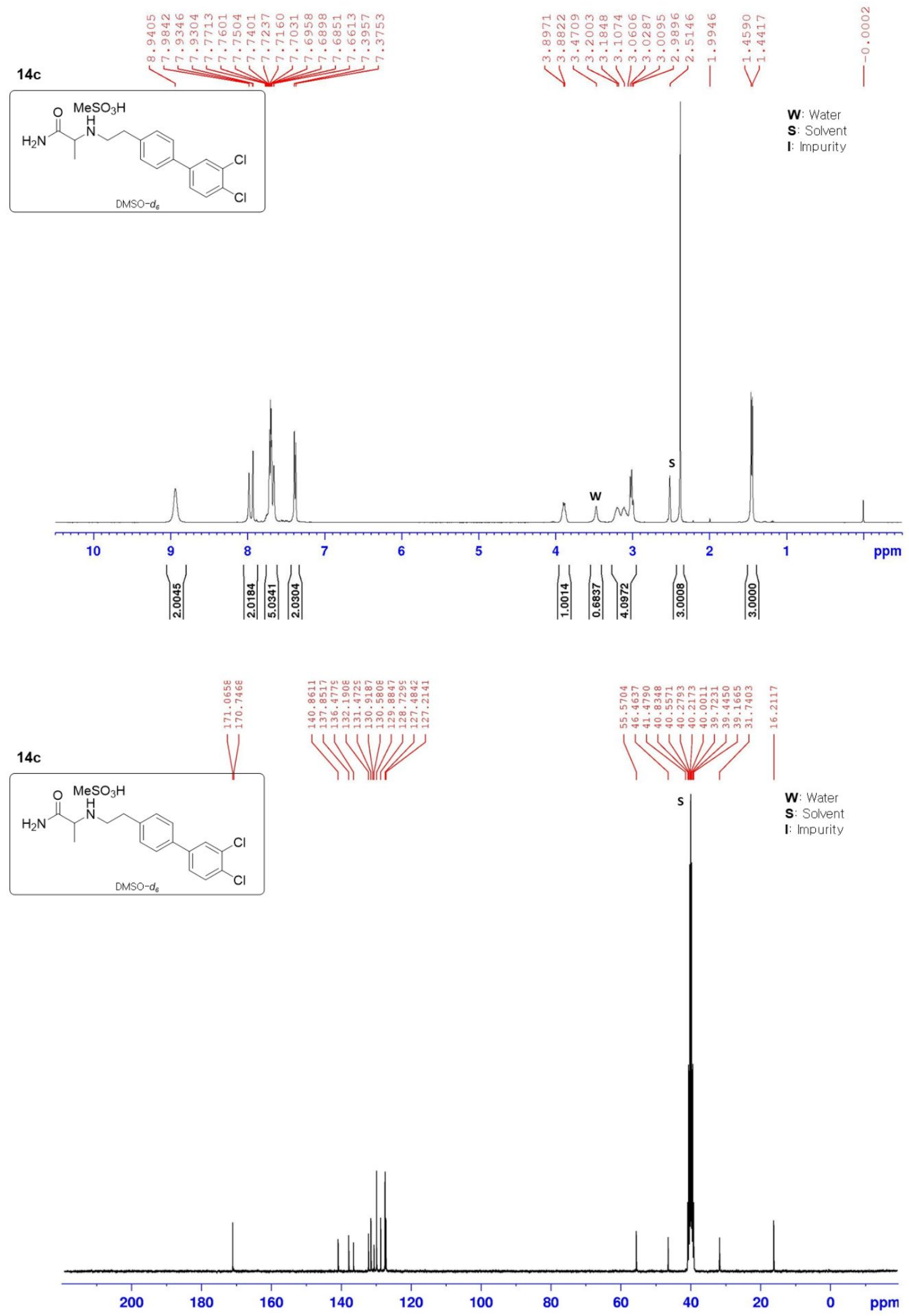

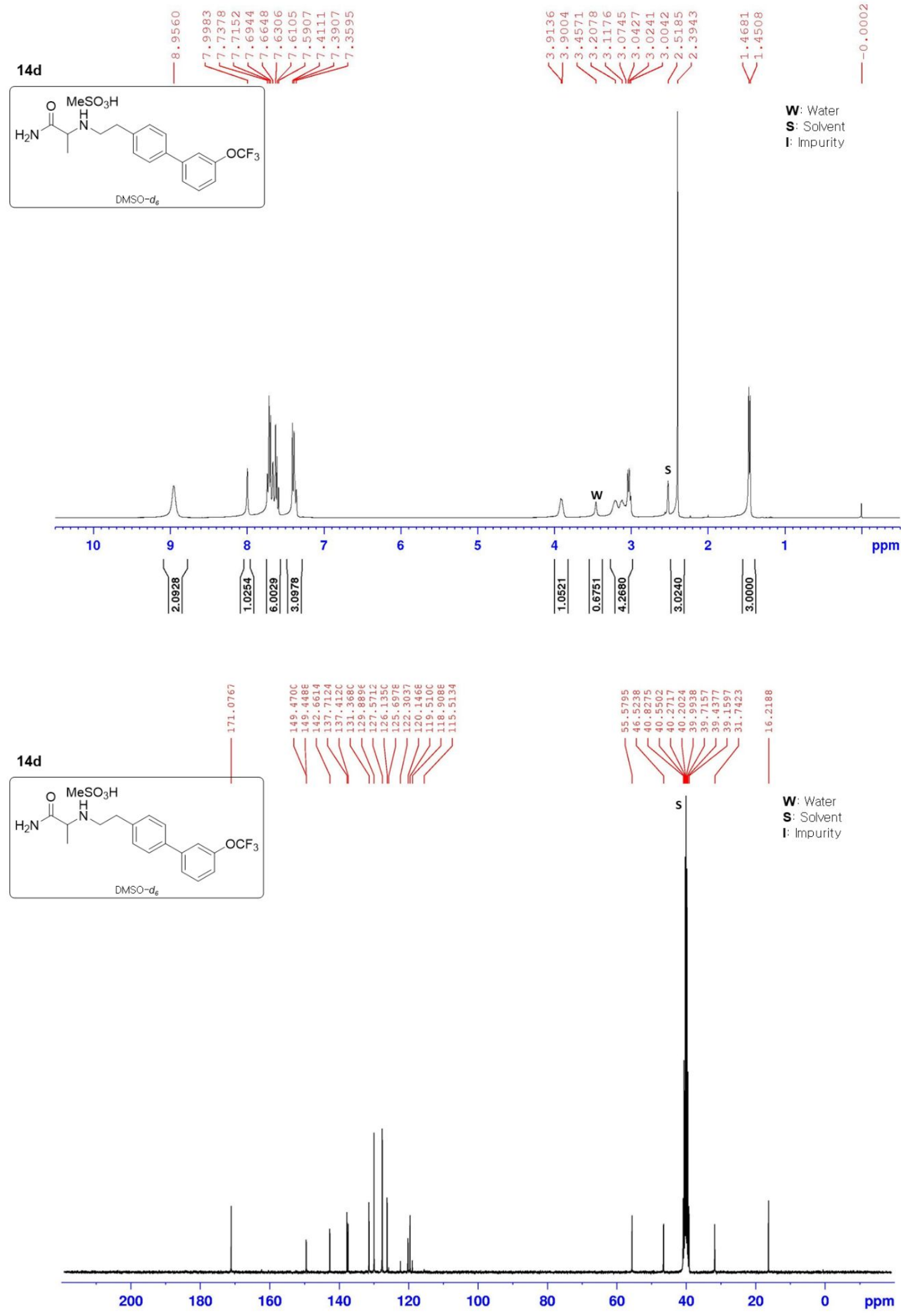

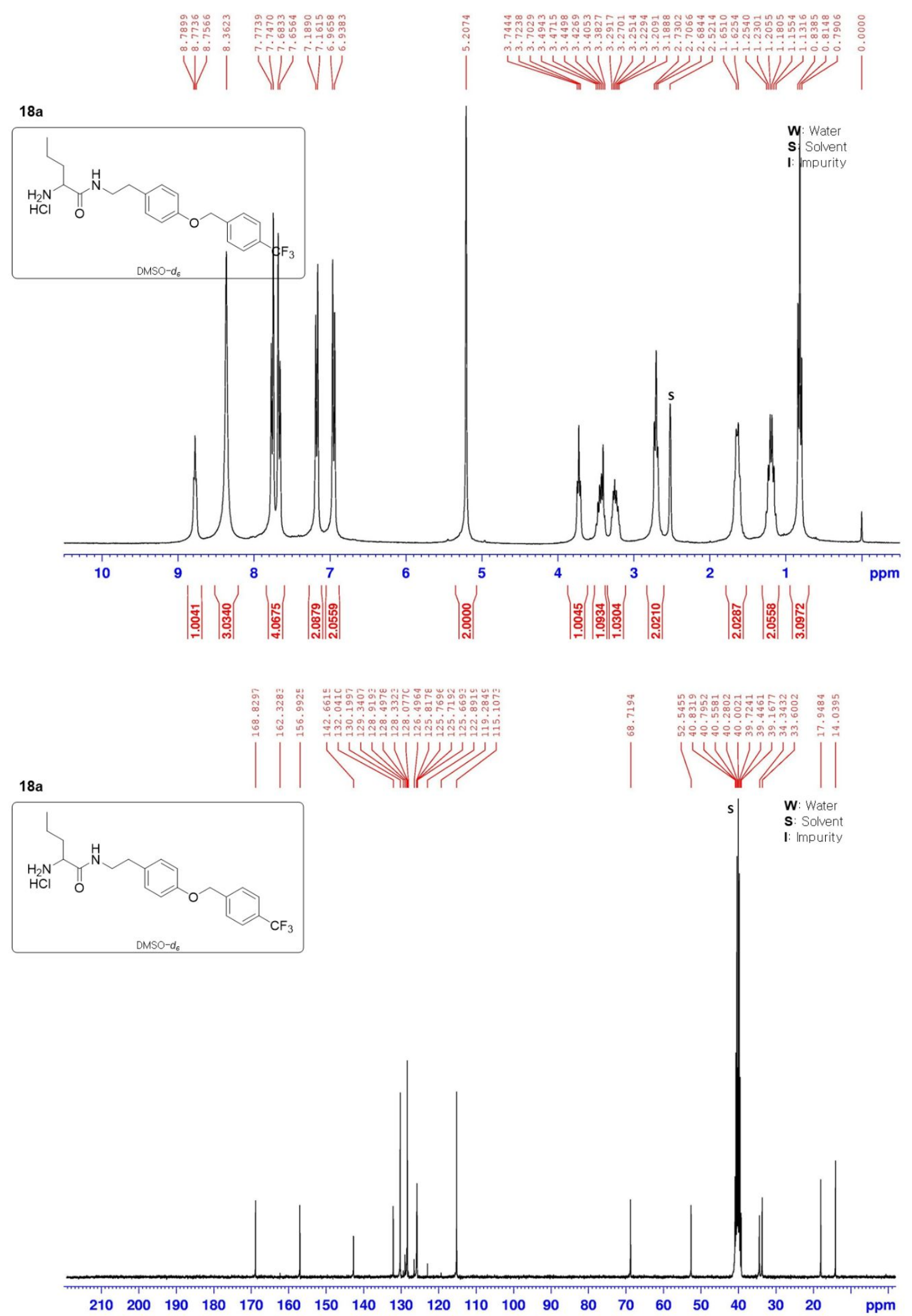


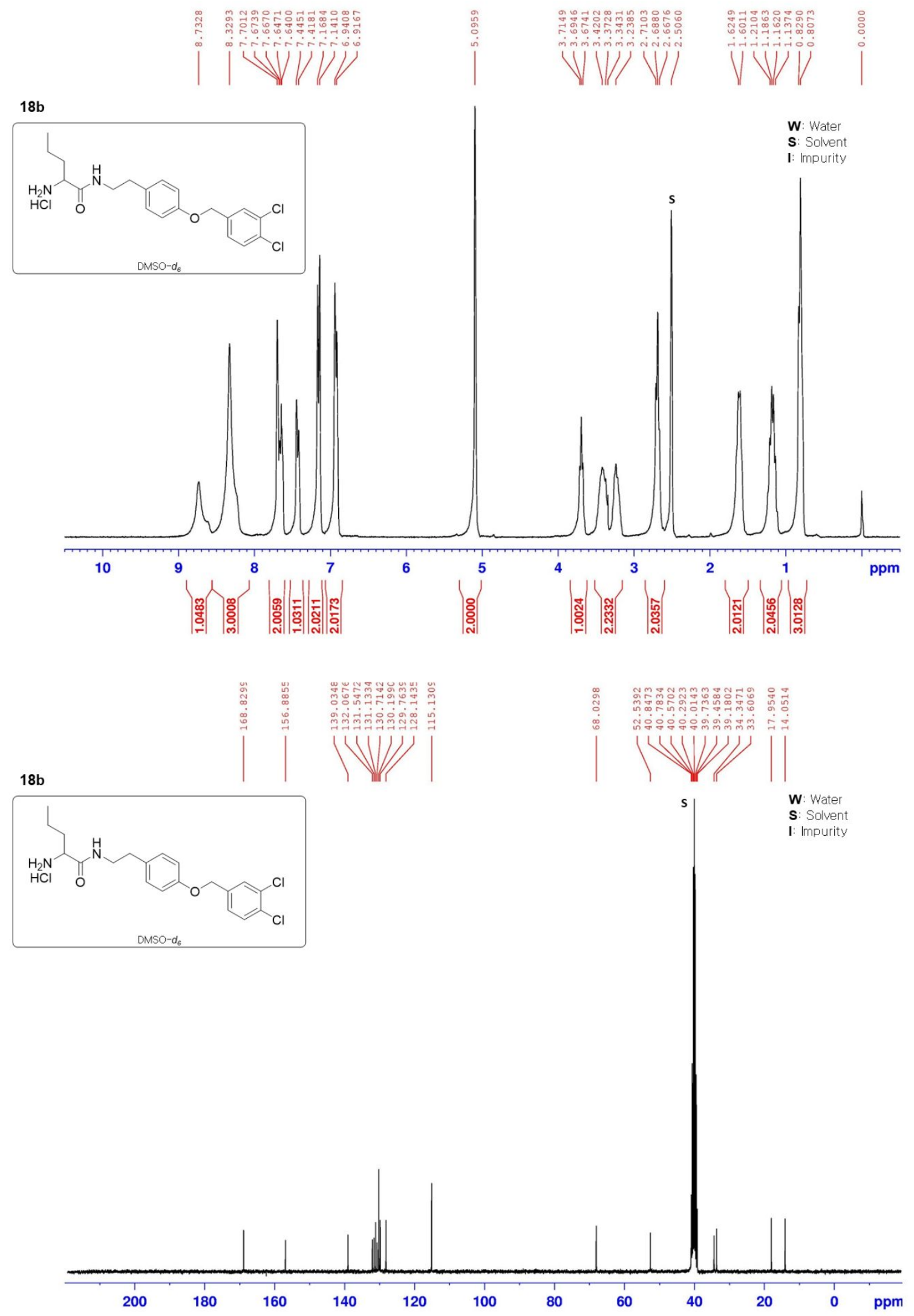



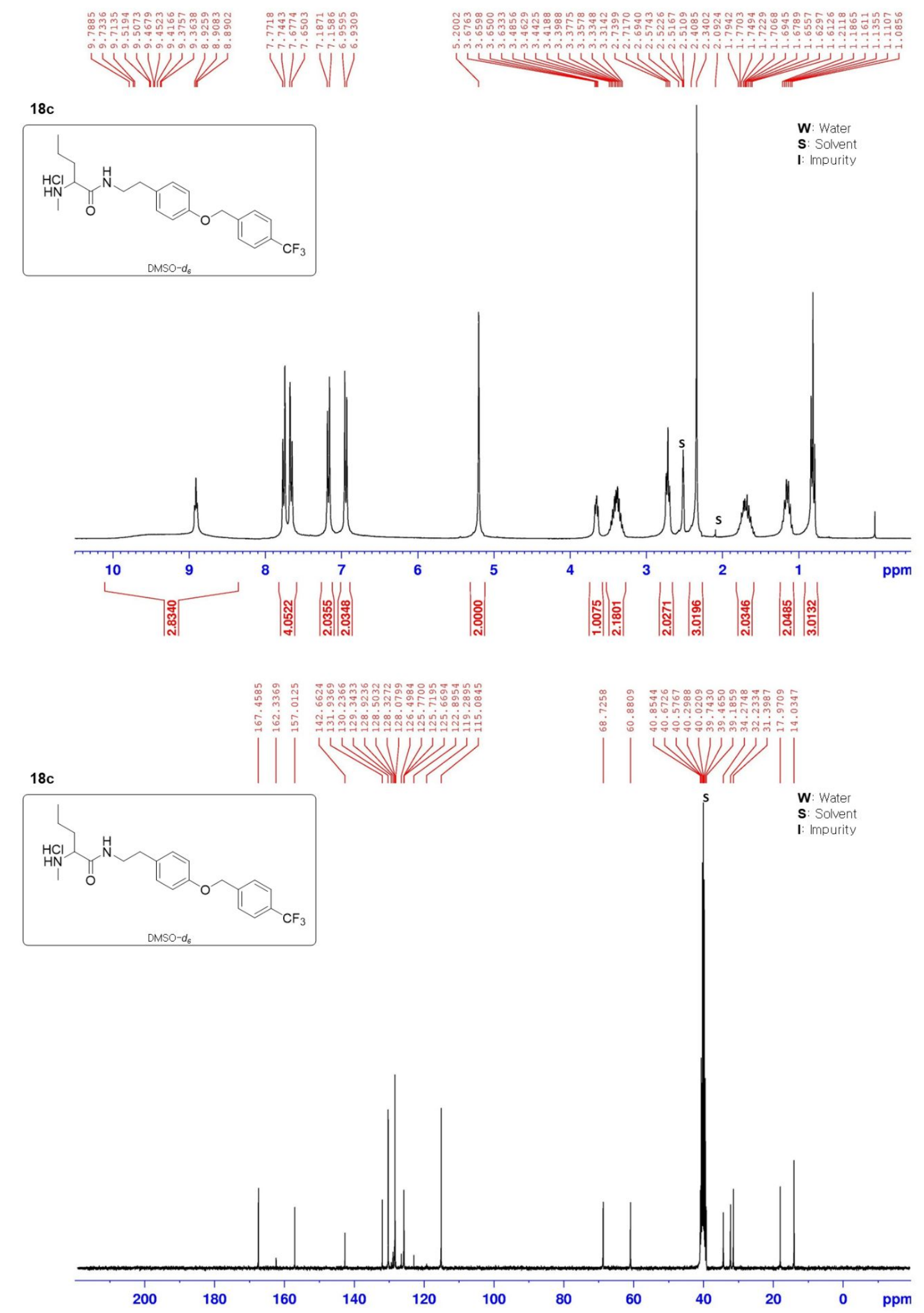

W: Water

S: Solvent

I: Impurity 

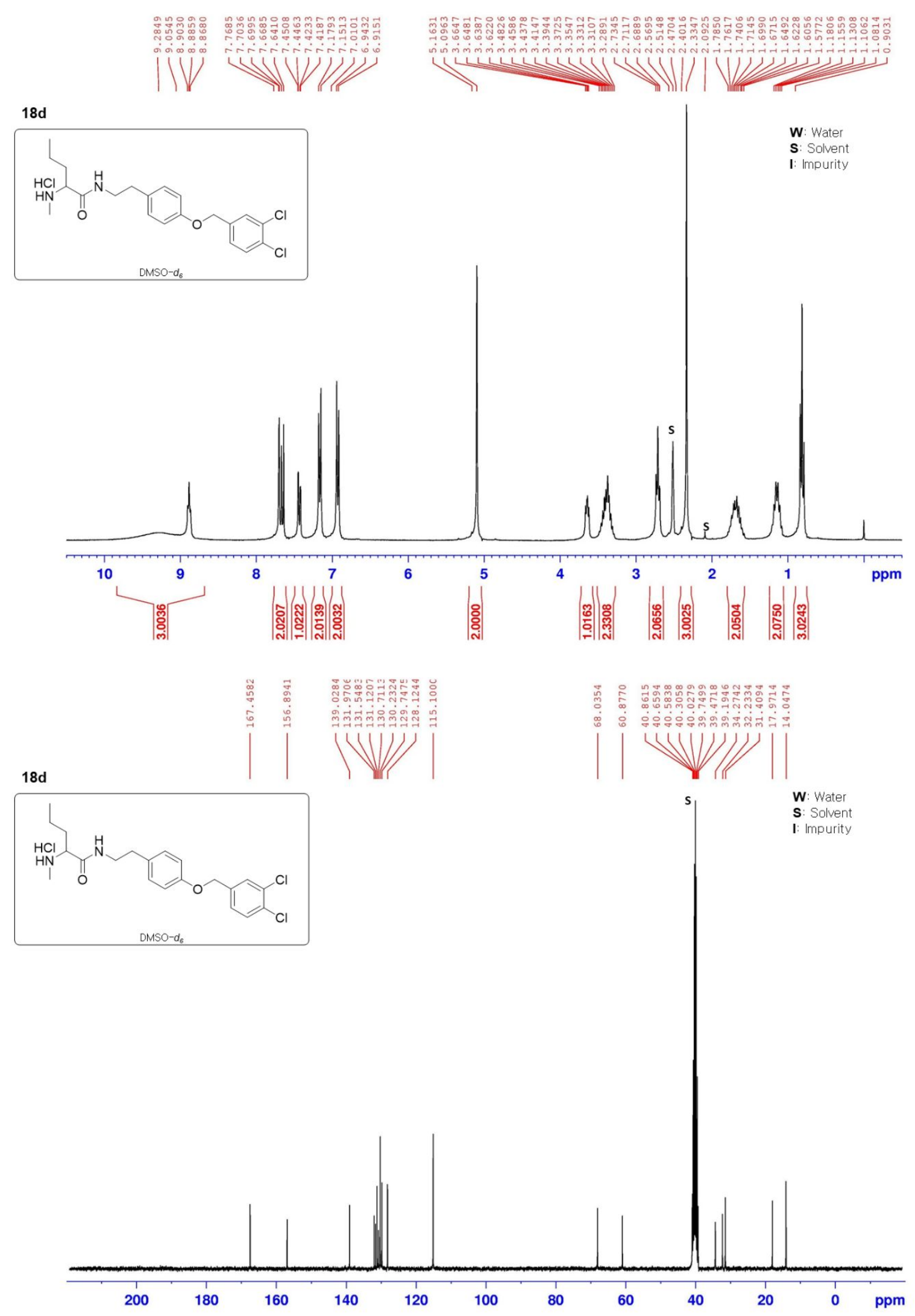


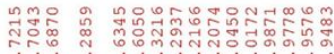

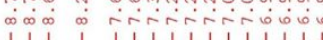

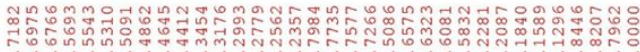

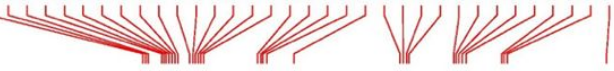

$20 \mathrm{a}$

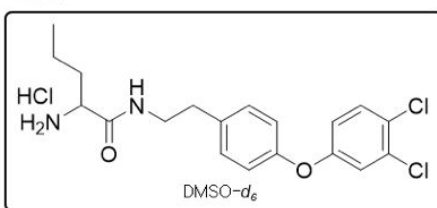

W: Water

S: Solvent

I: Impurity

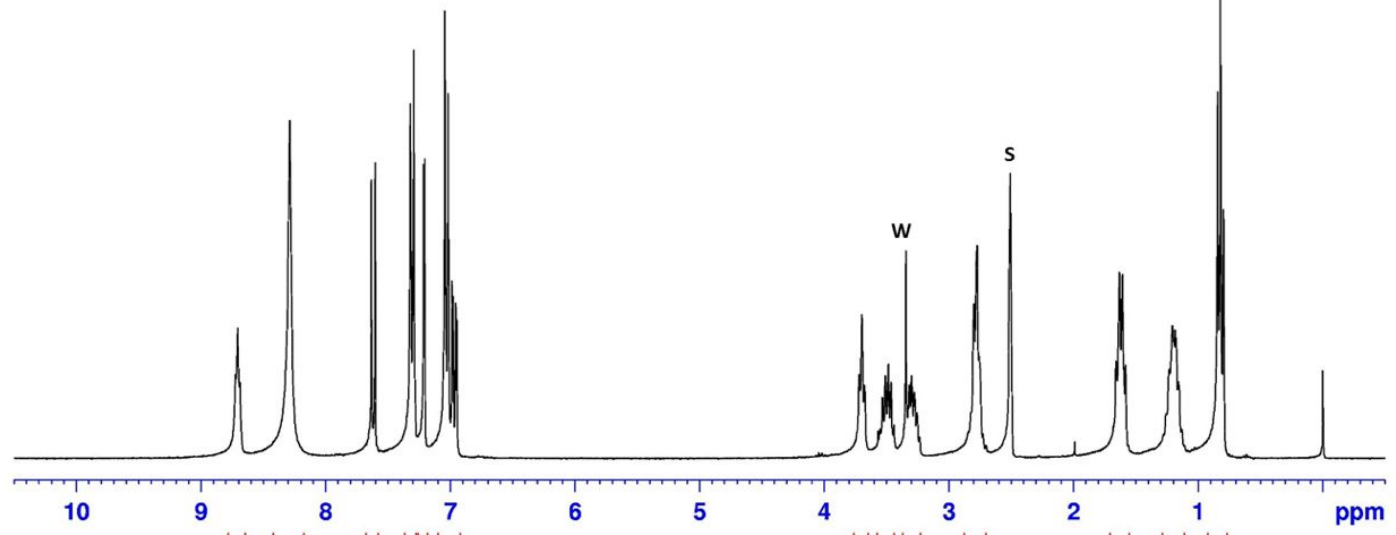

|

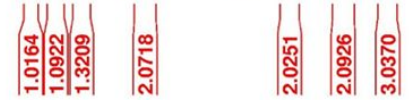

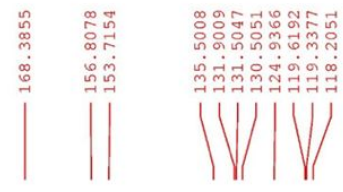

$20 \mathrm{a}$

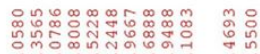

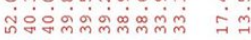

10.1/W

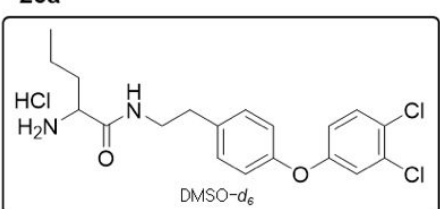

S: Solvent

I: Impurity

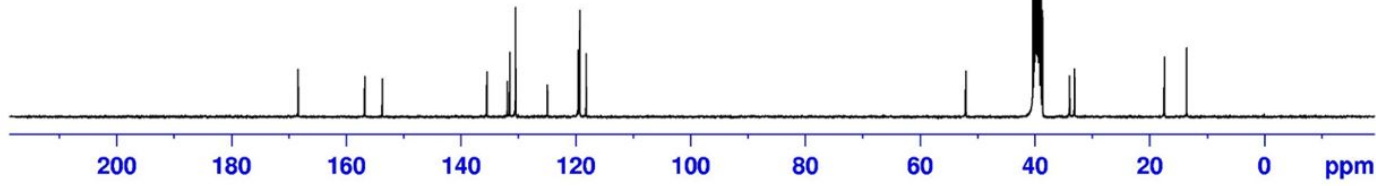


20b

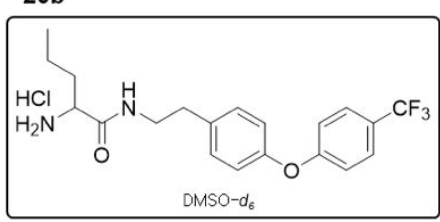

W: Water

S: Solvent

I: Impurity
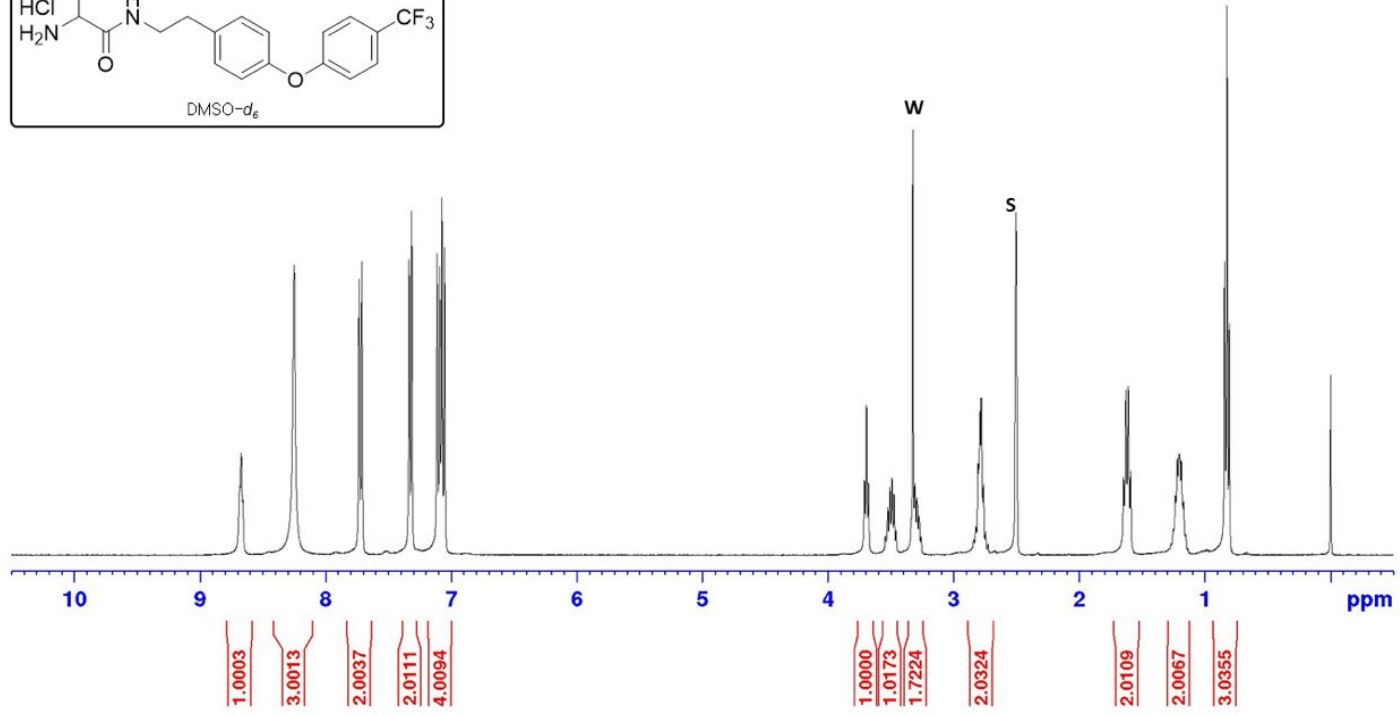

|

|ำ

ㅎํำ

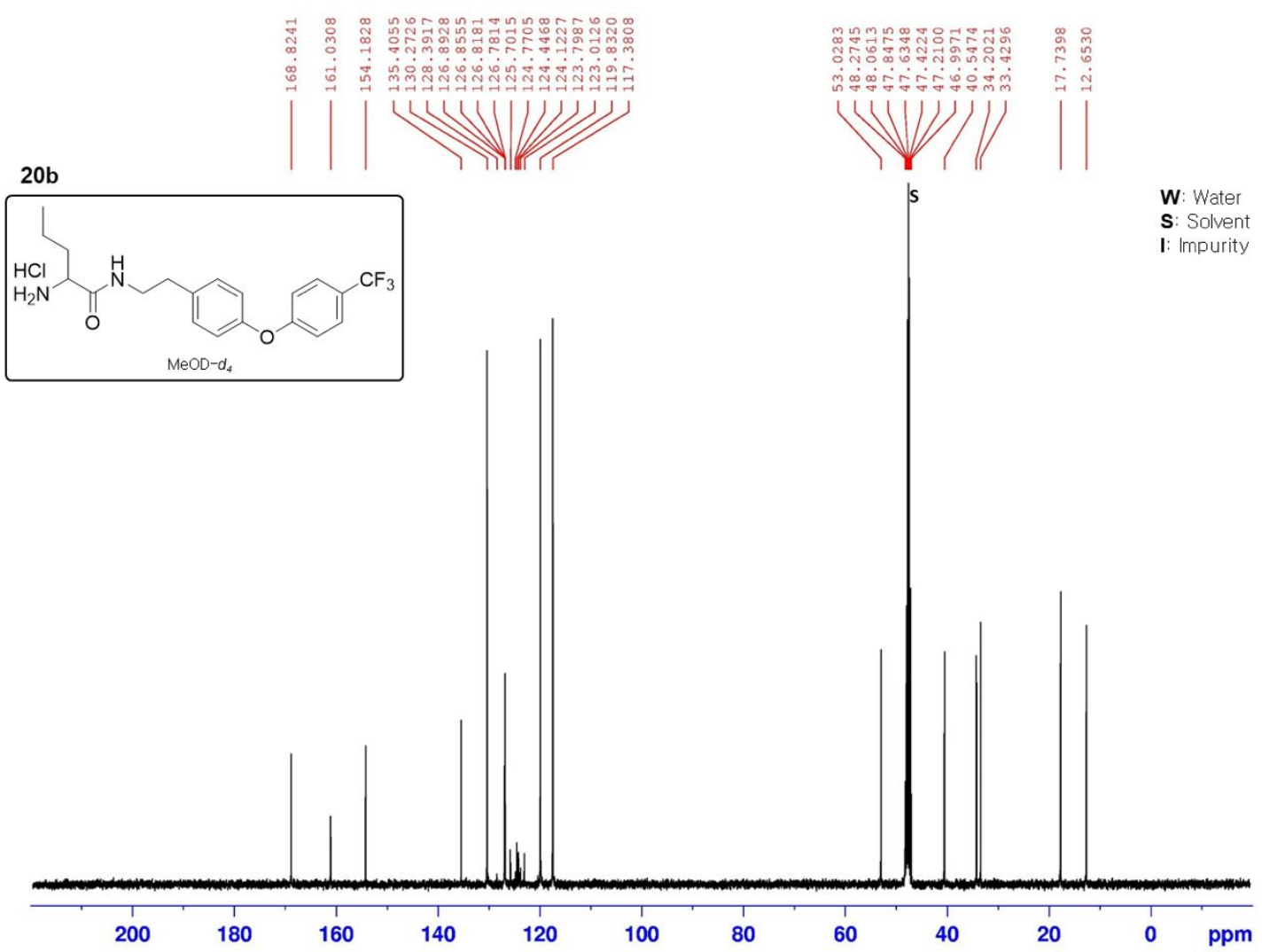




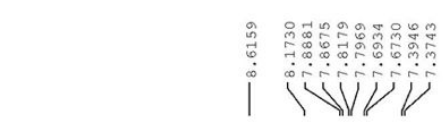

$22 a$

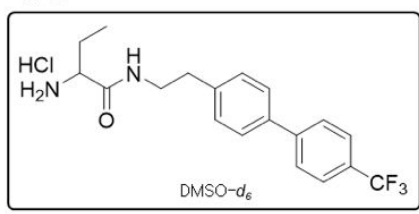

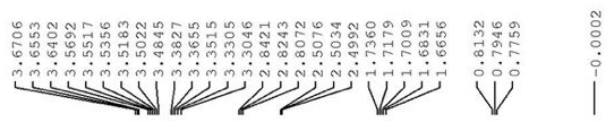

w

W: Wate

S: Solvent

I: Impurity
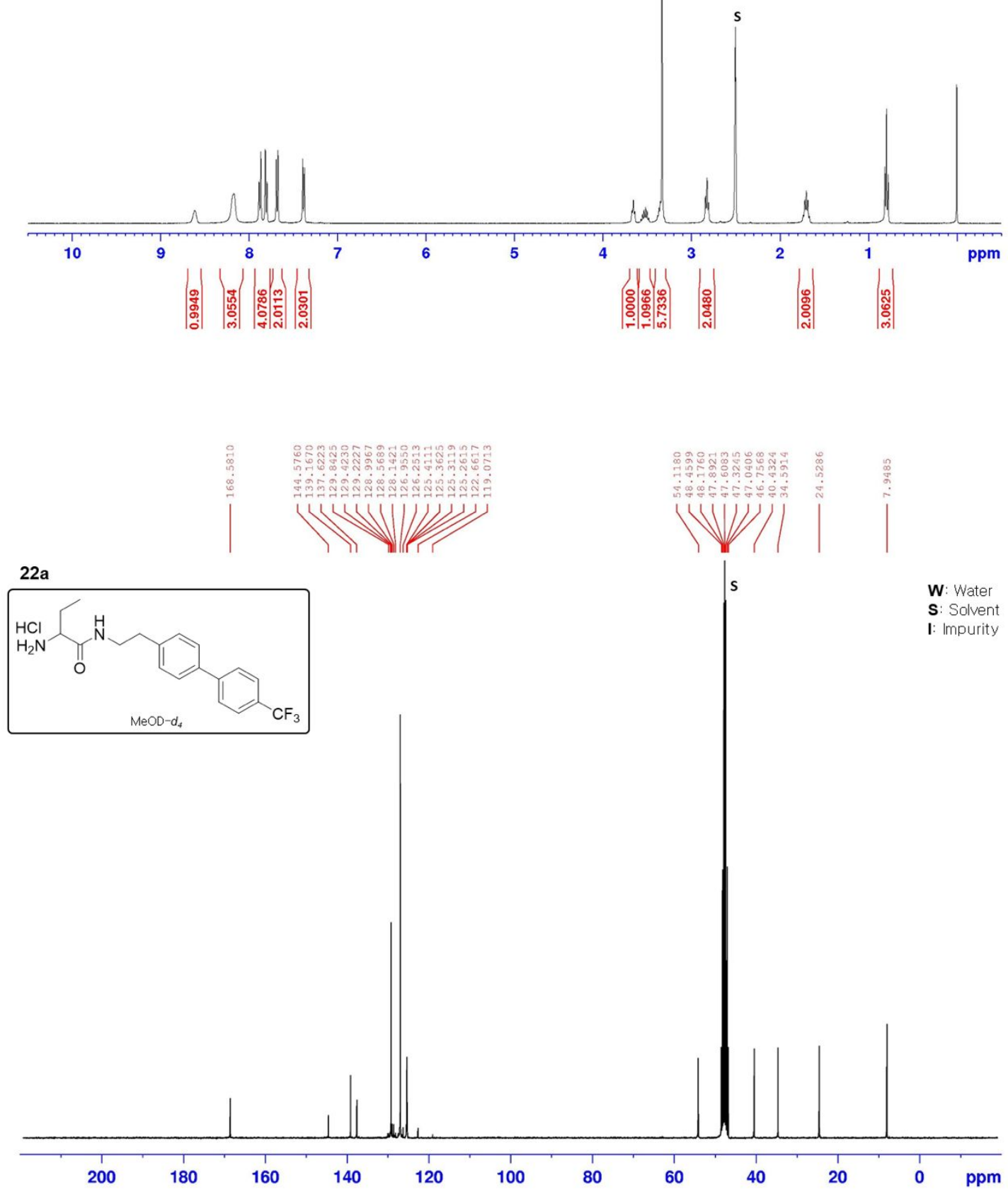

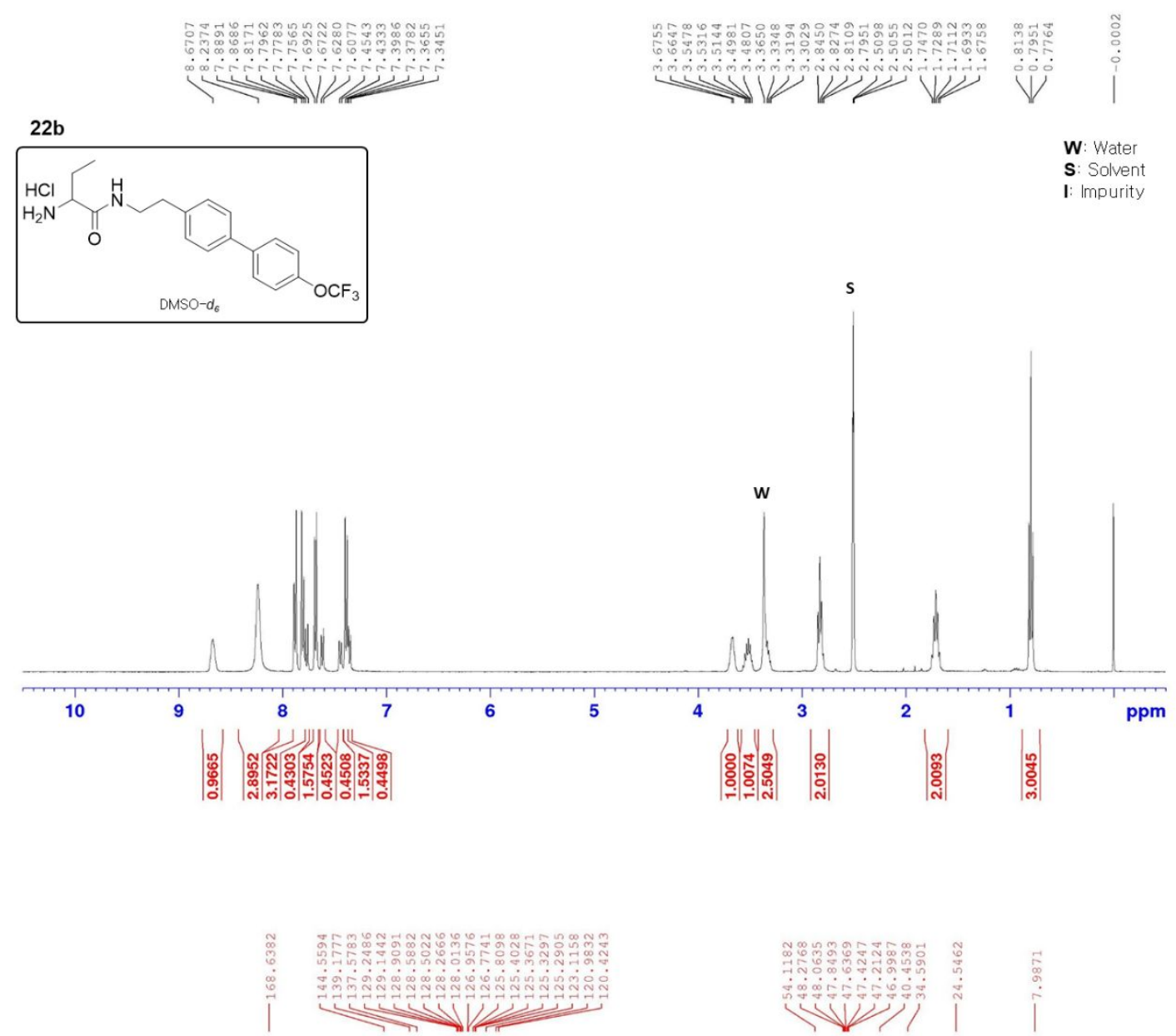

22b
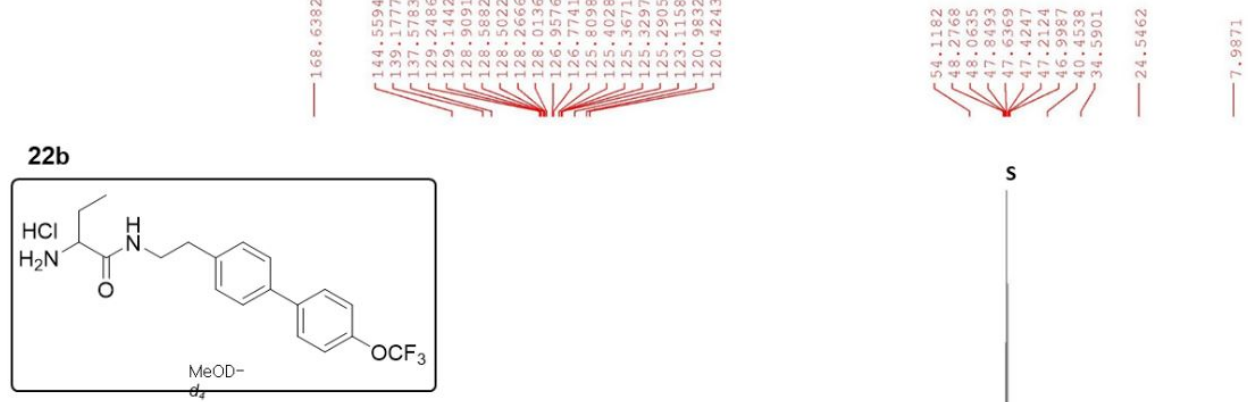

W: Water

S: Solvent

I: Impurity

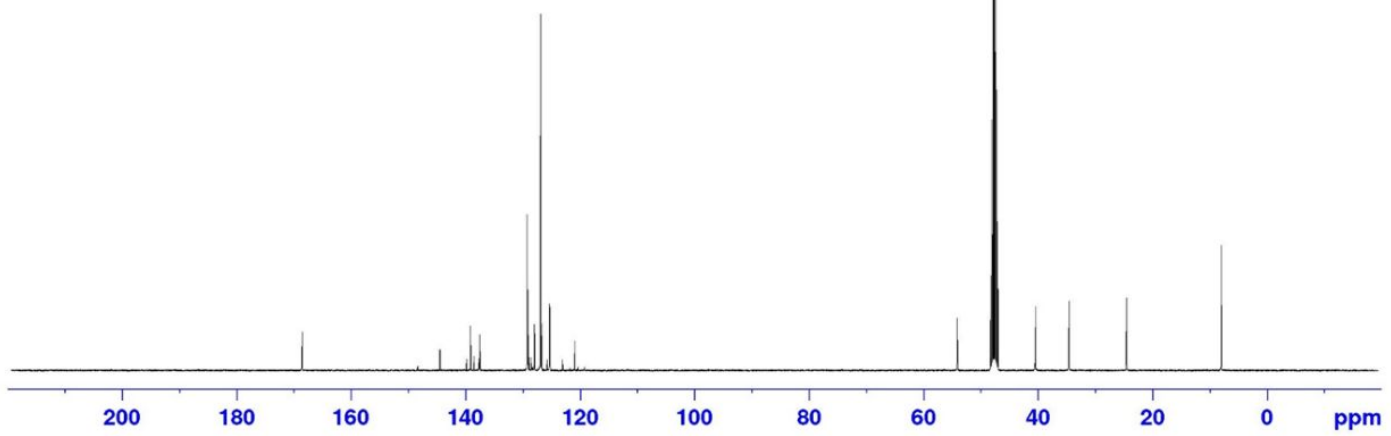



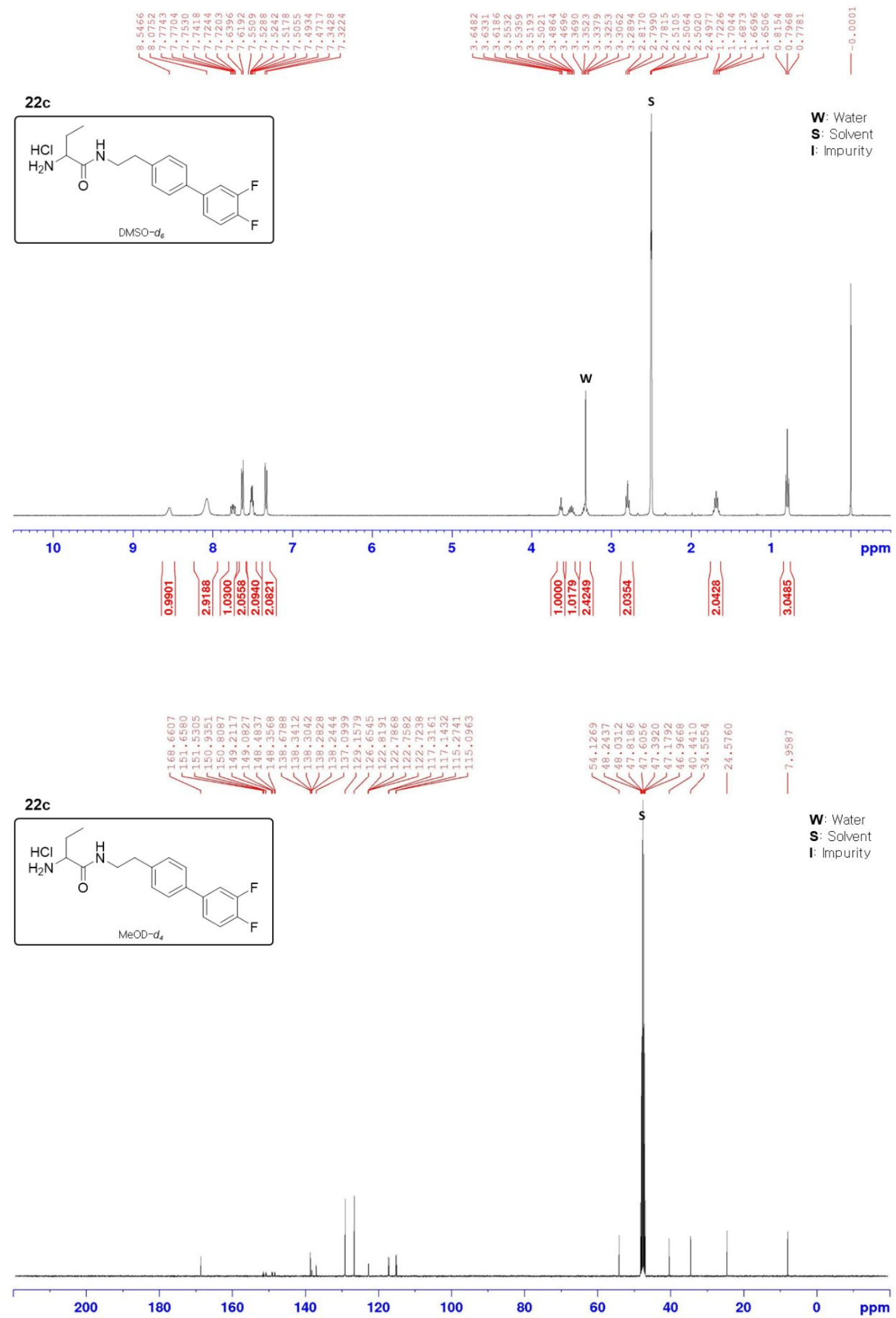


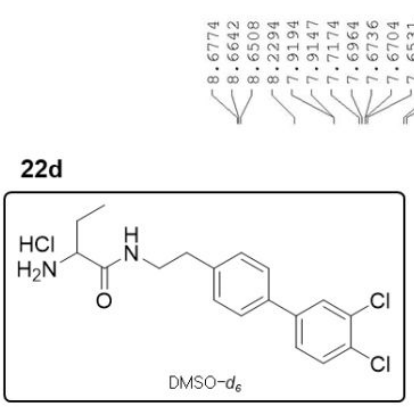

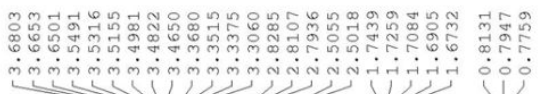

W: Water

S: Solvent

I: Impurity
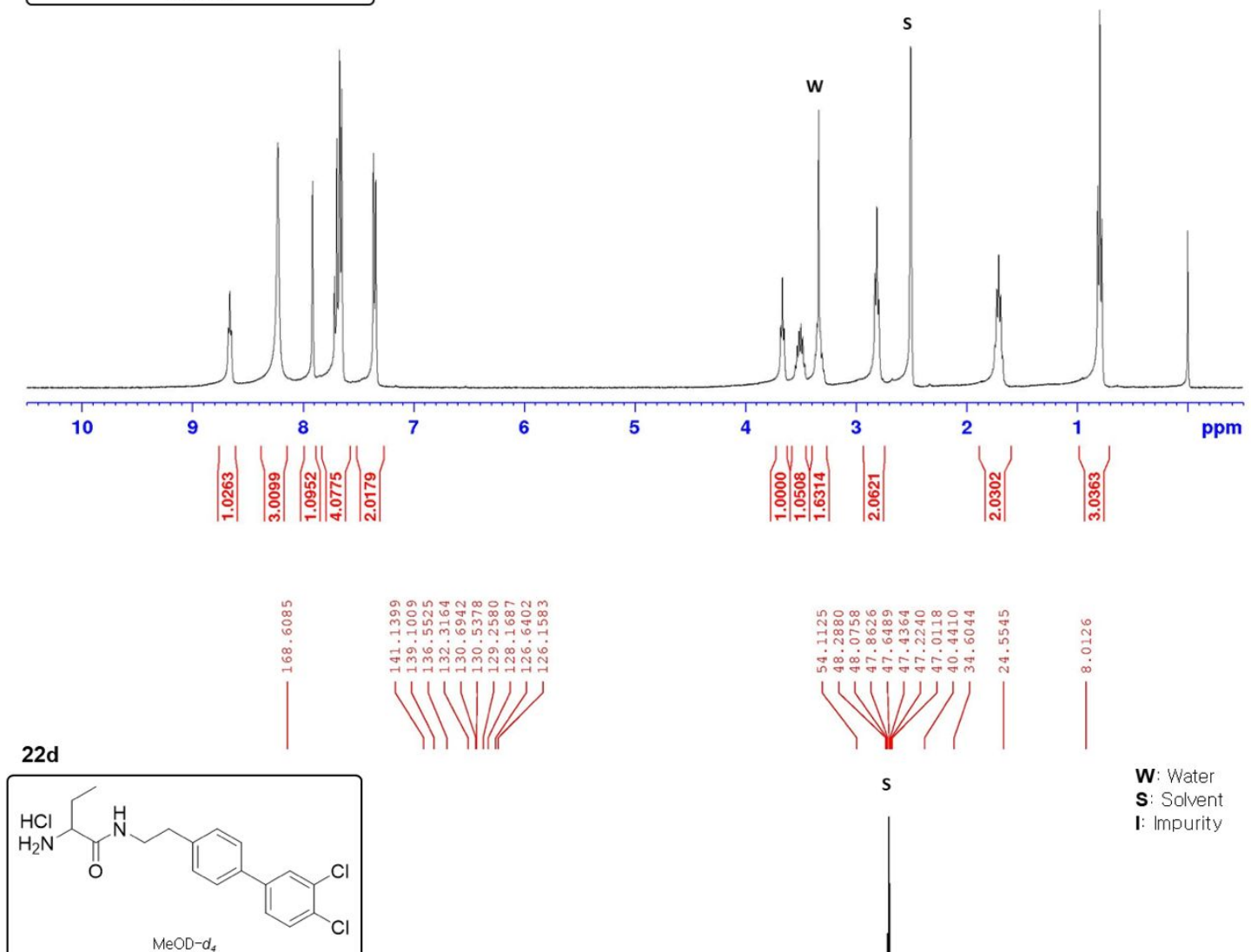

W: Water

S: Solvent

I: Impurity

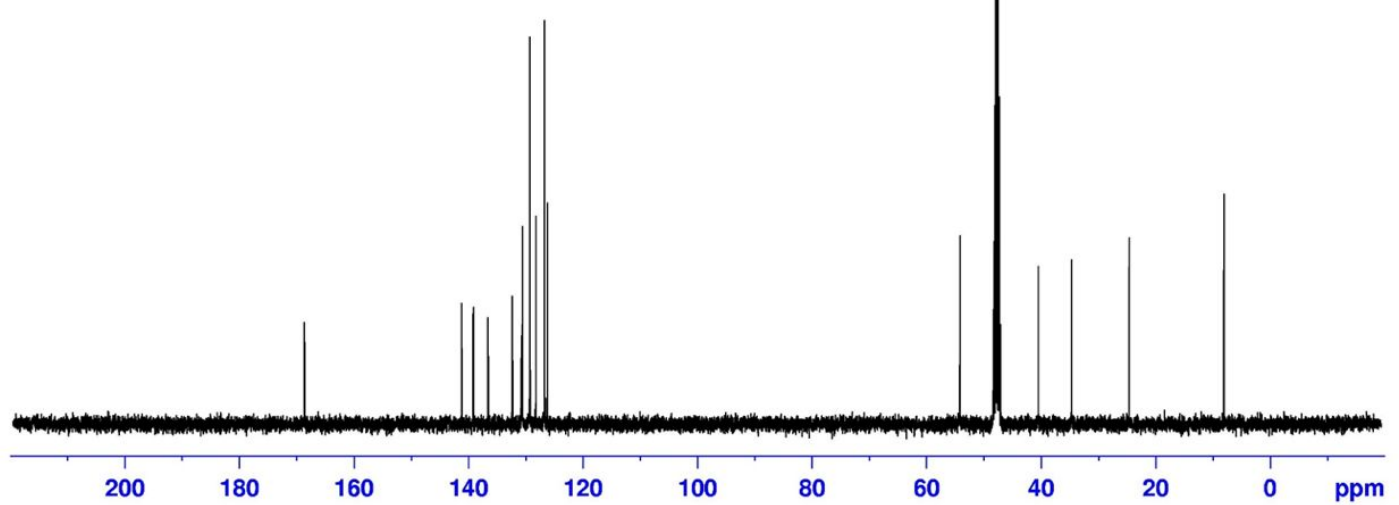



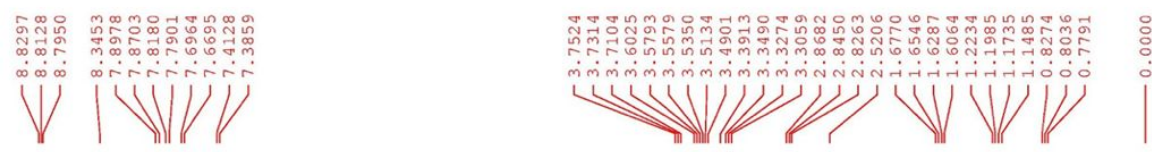

22

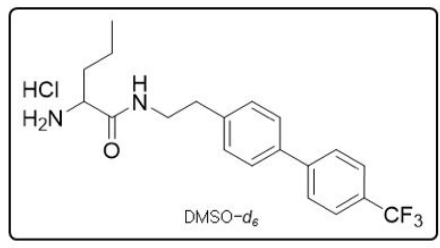

S: Solvent I: Impurity
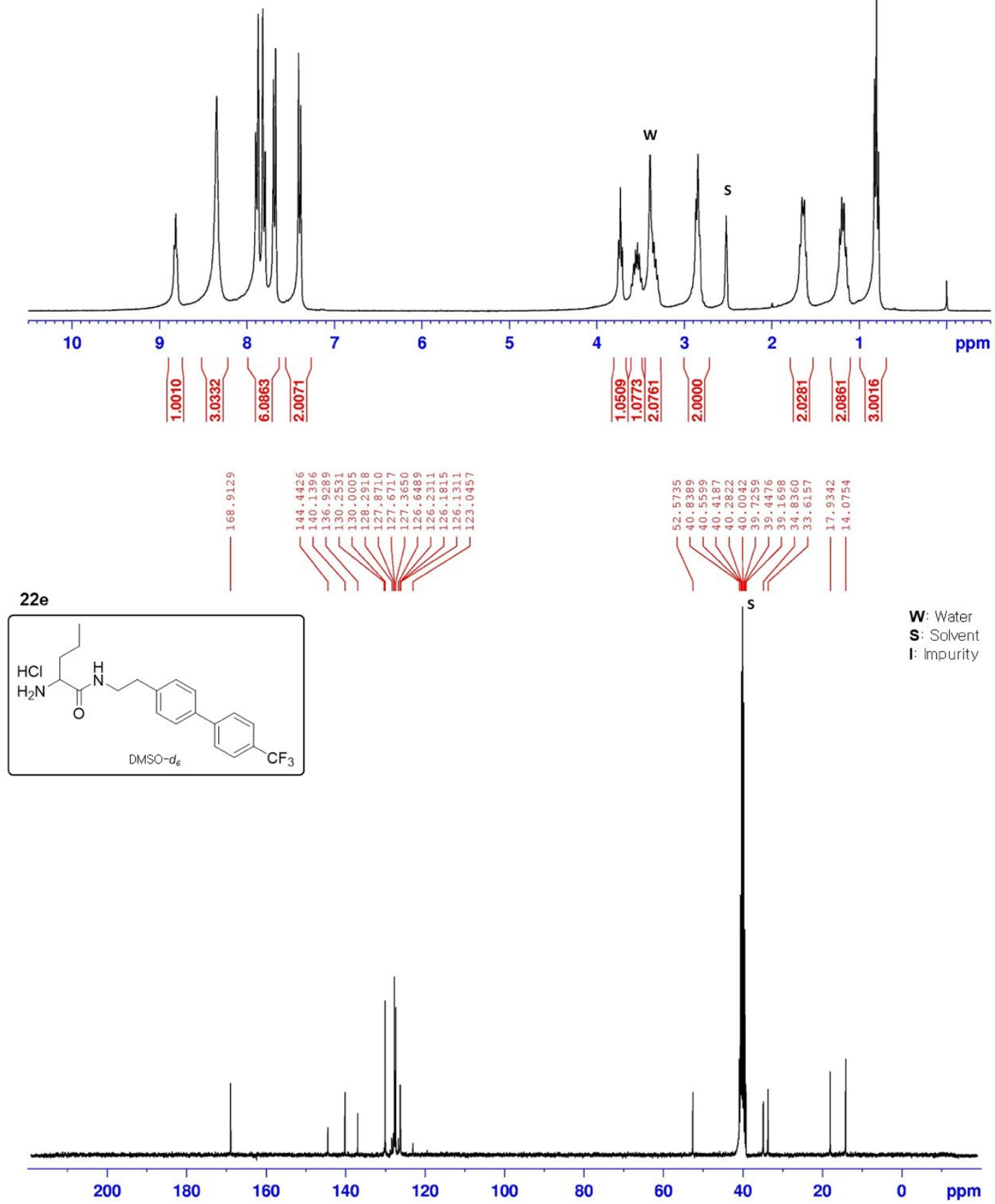

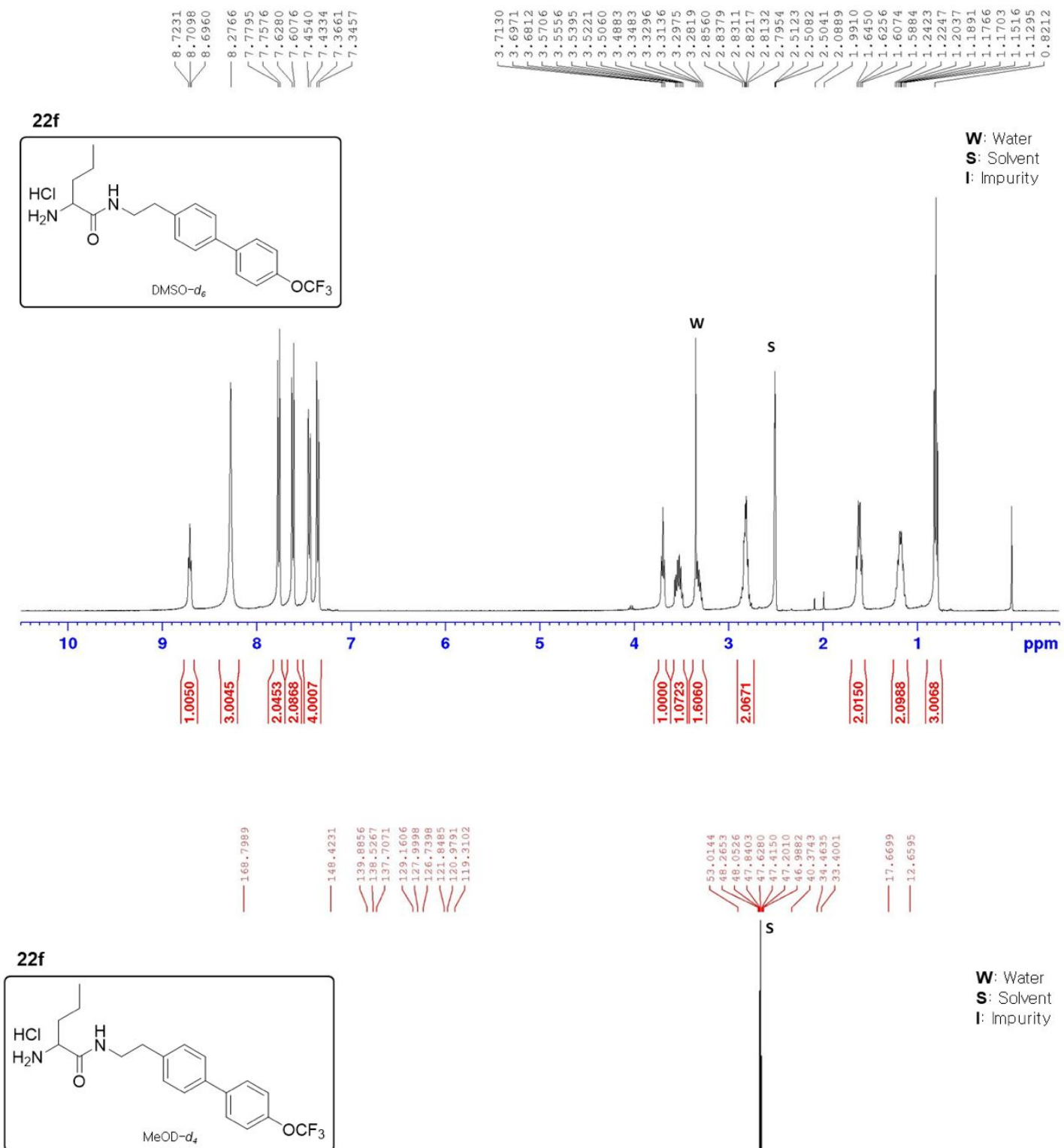

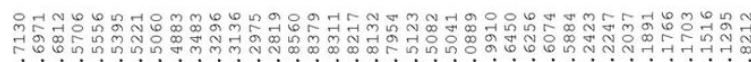

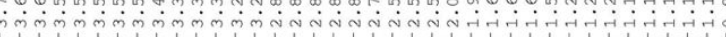

W: Water

S: Solvent

I: Impurity

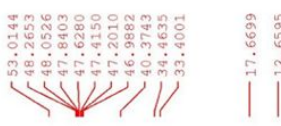

s

W: Water

S: Solvent

I: Impurity

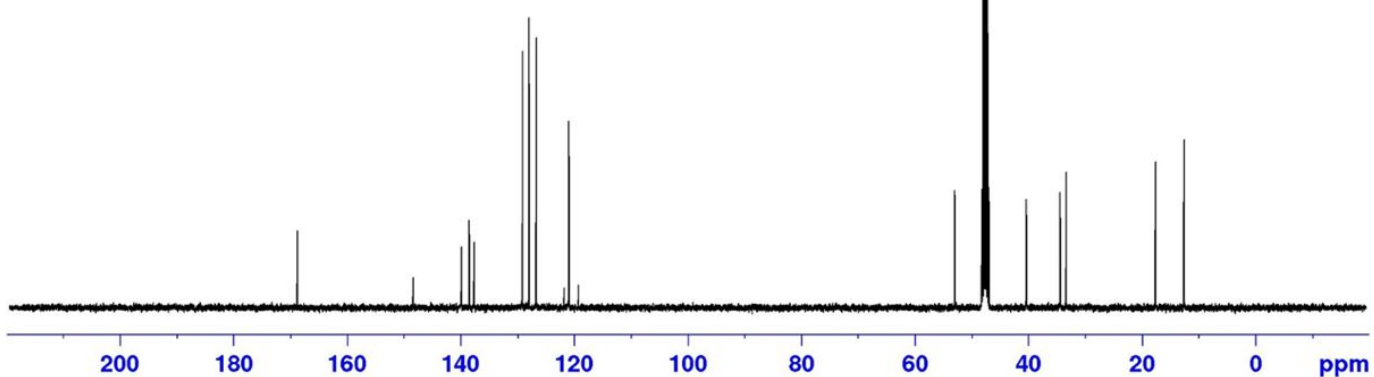



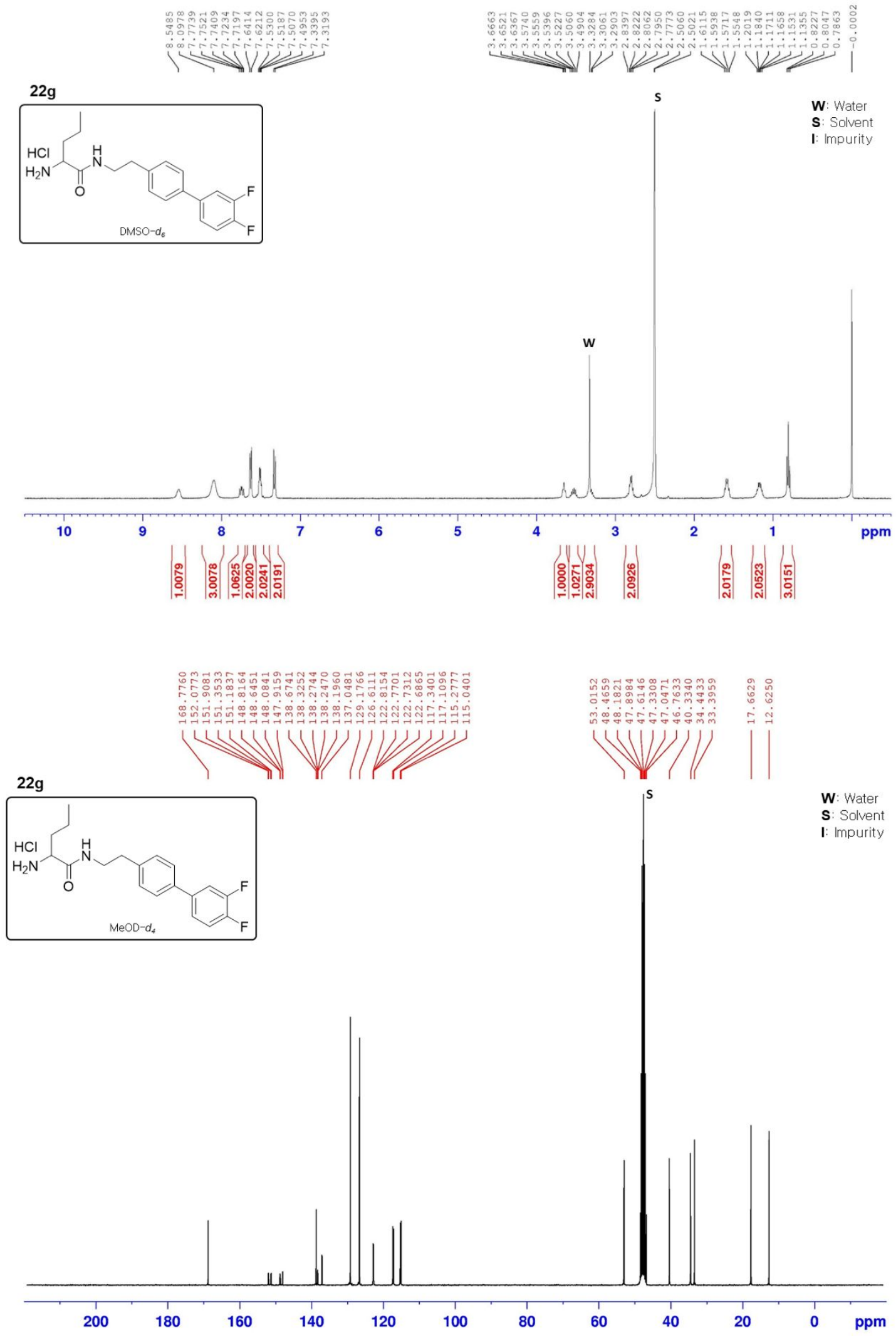


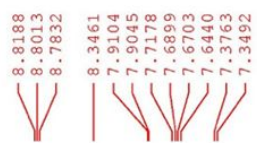

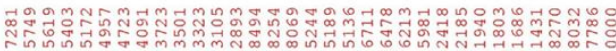
? (n)

$22 \mathrm{~h}$

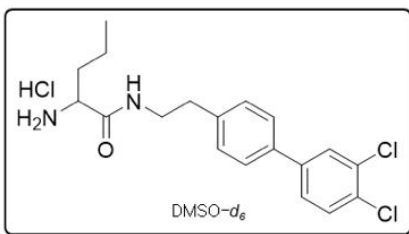

W: Water

S: Solvent

I: Impurity
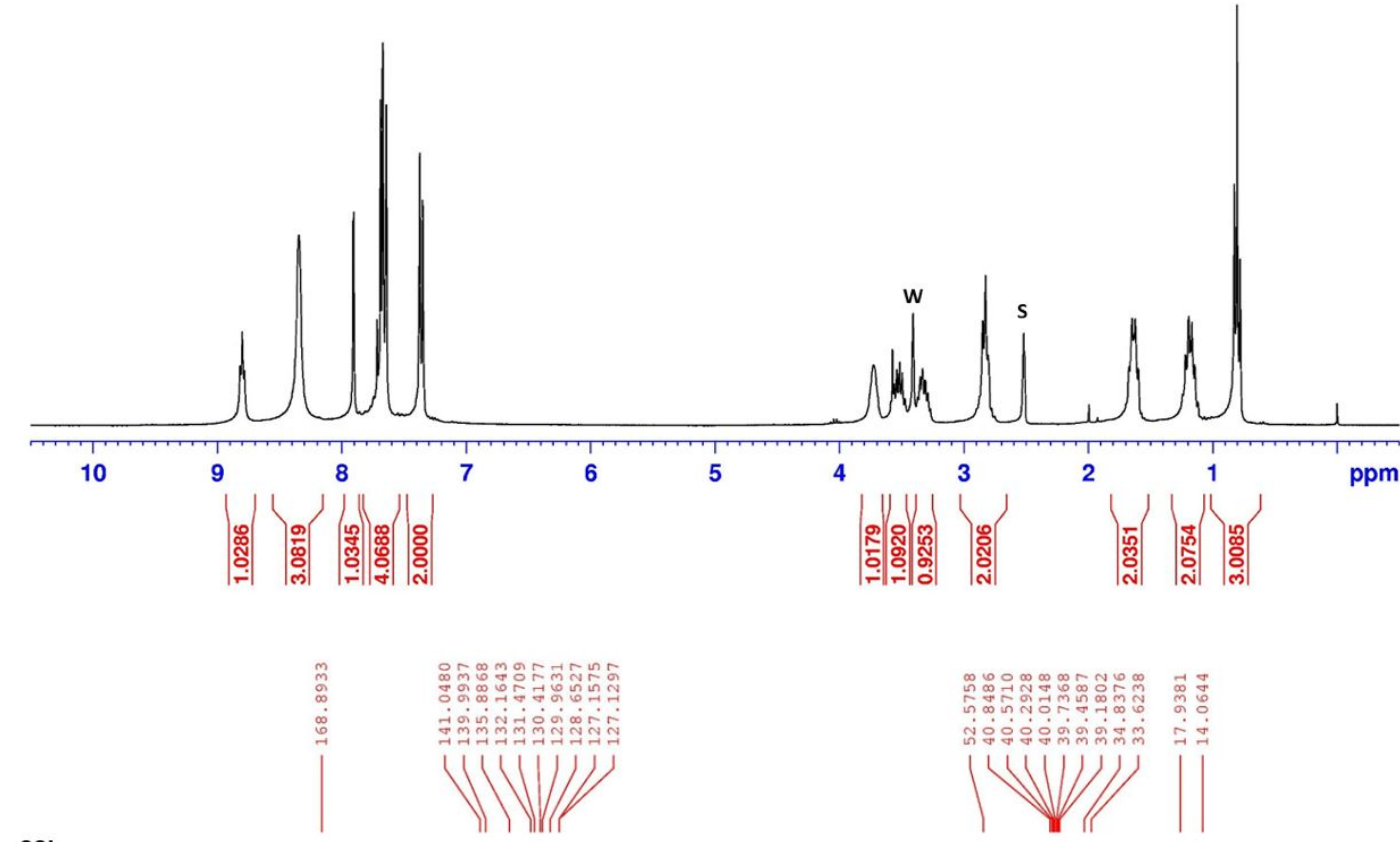

$22 \mathrm{~h}$
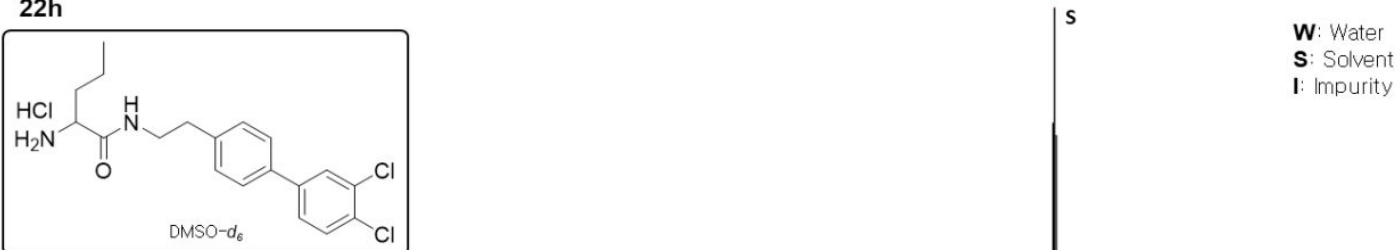

S: Solvent

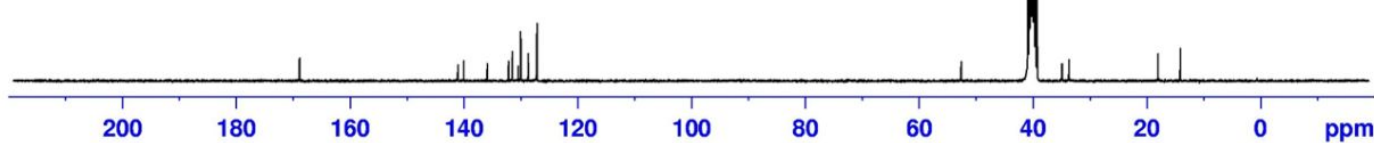




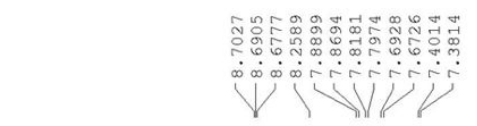

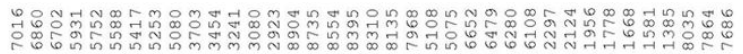

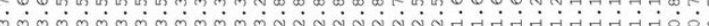

22
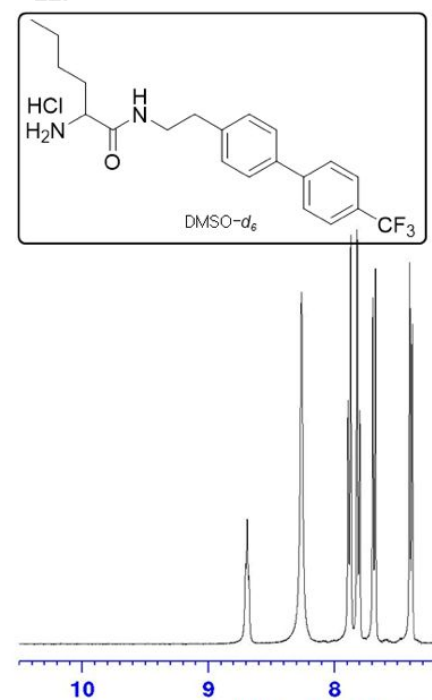
|
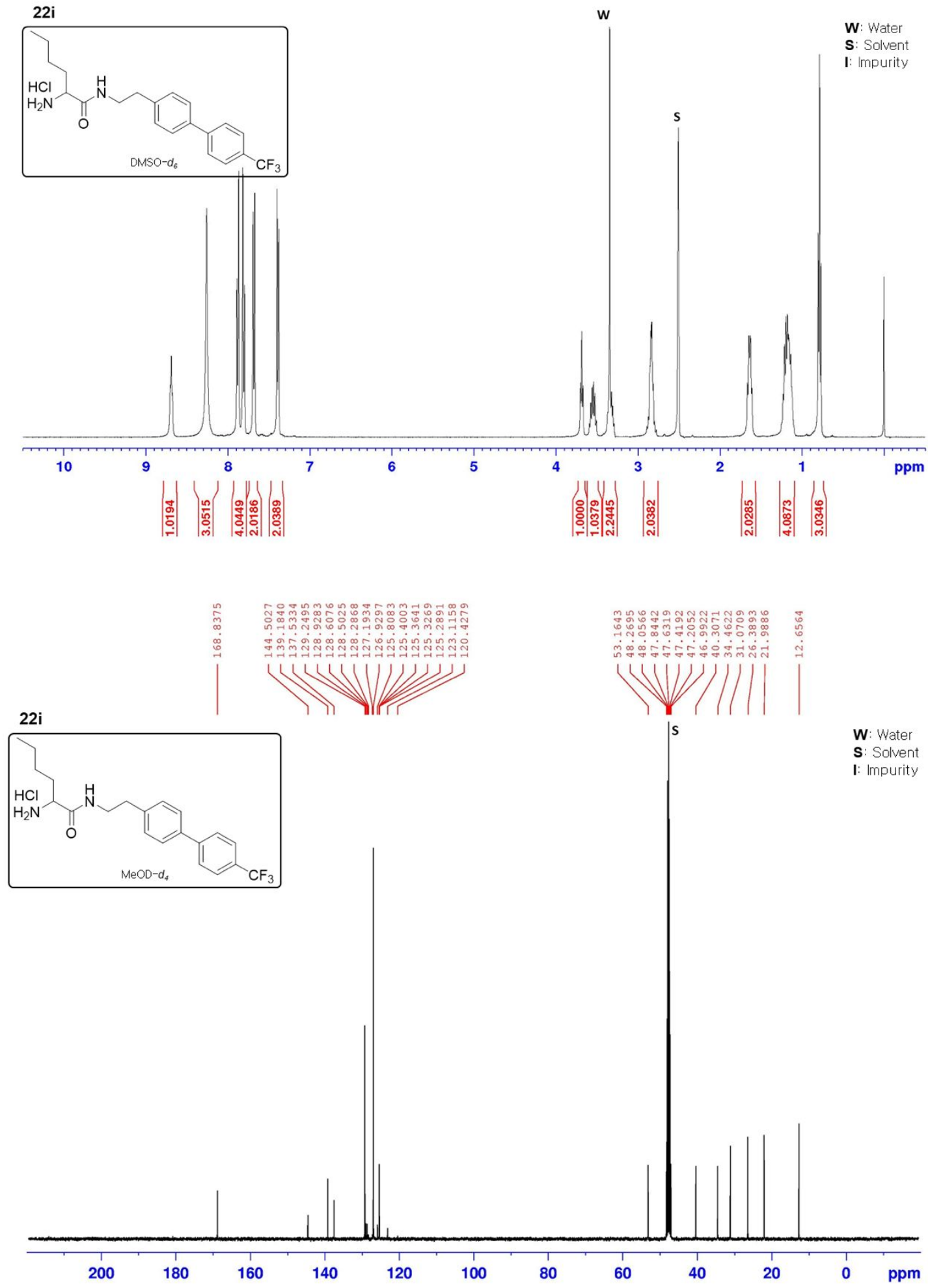

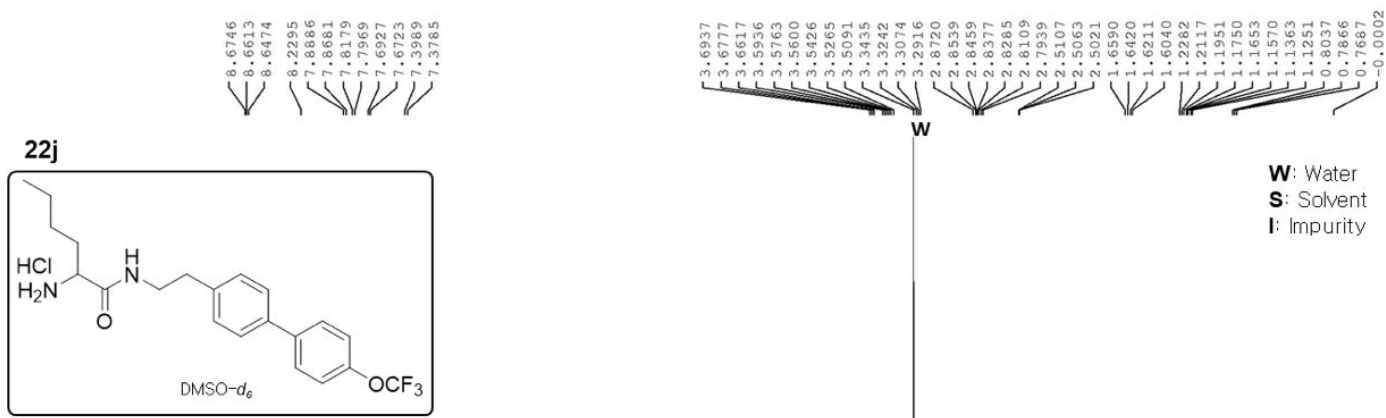

W: Water
$\mathbf{S}$ : Solvent

S: Solvent

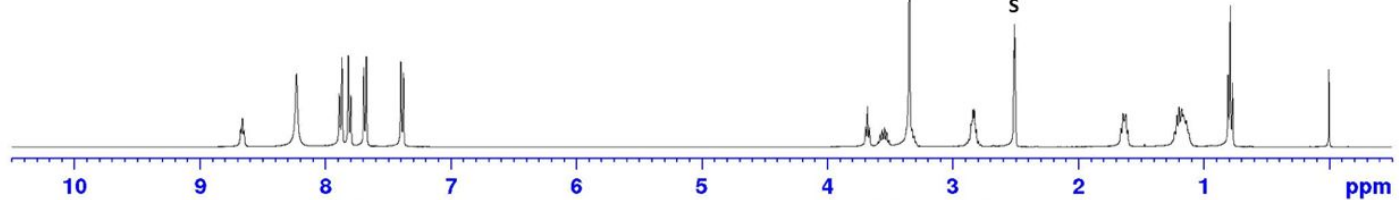

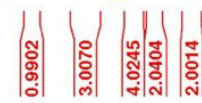

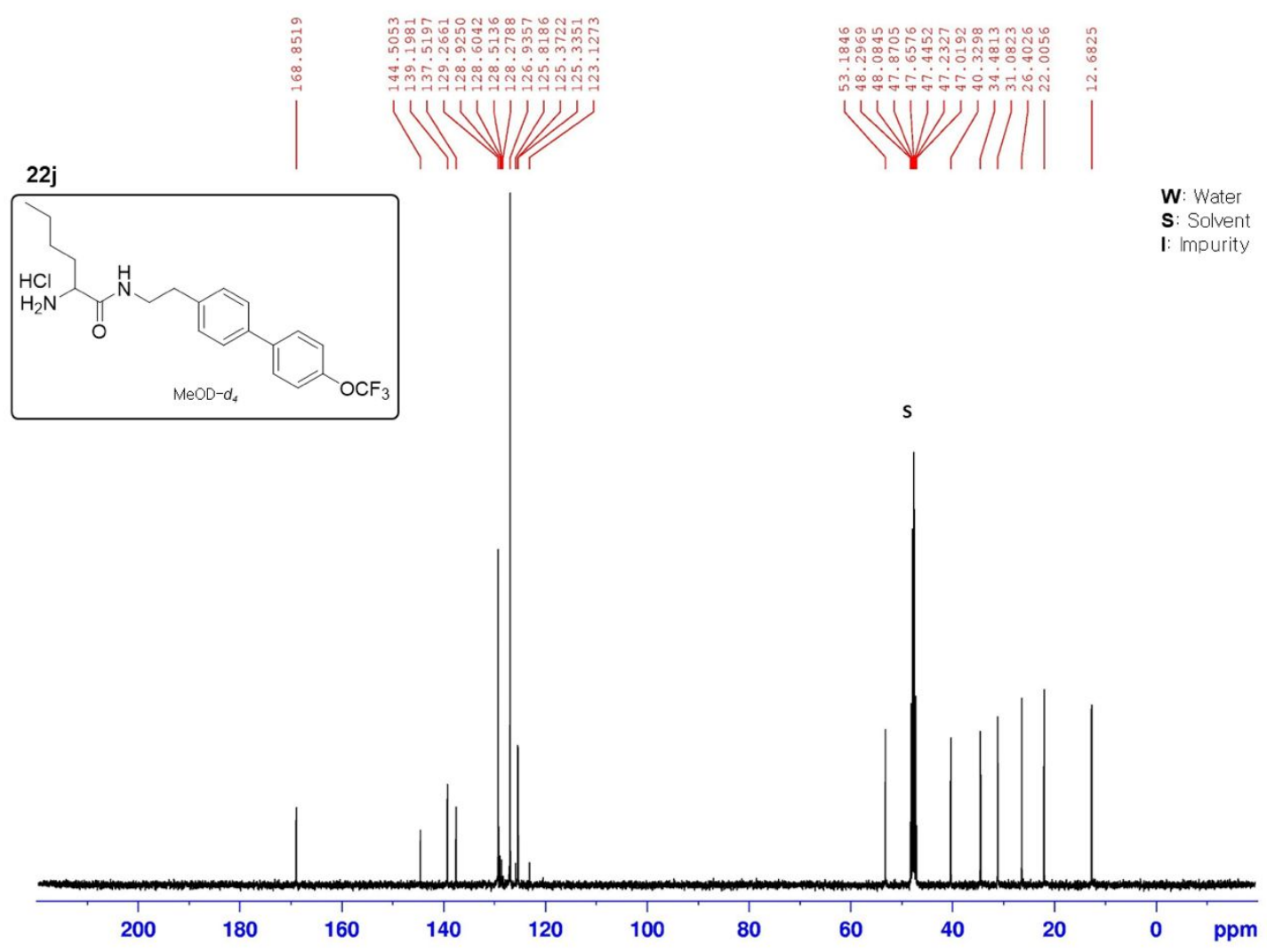



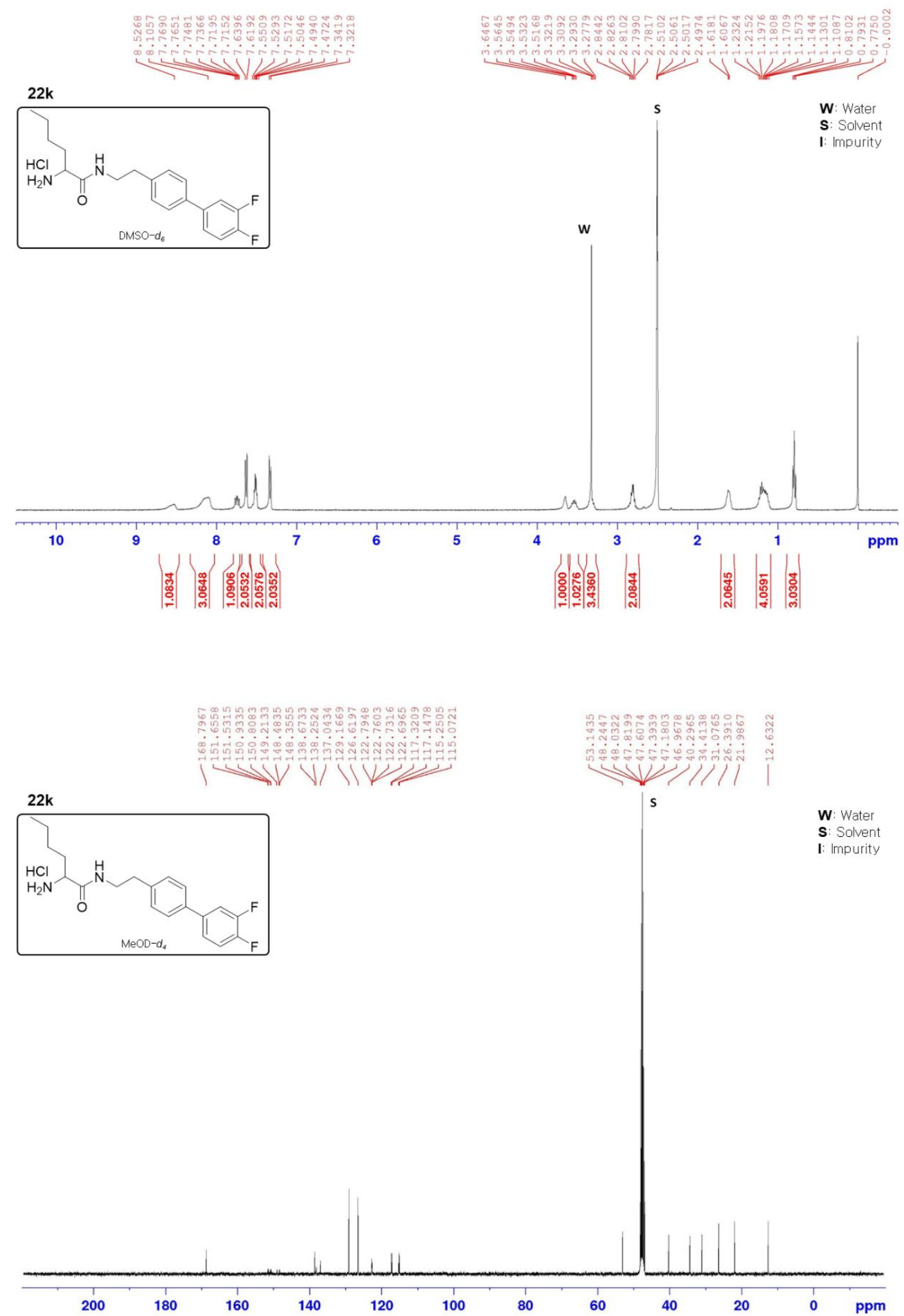


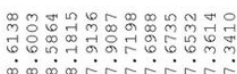

Vं

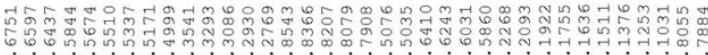

221

W: Water

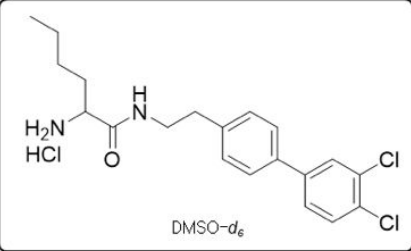

I: Impurity

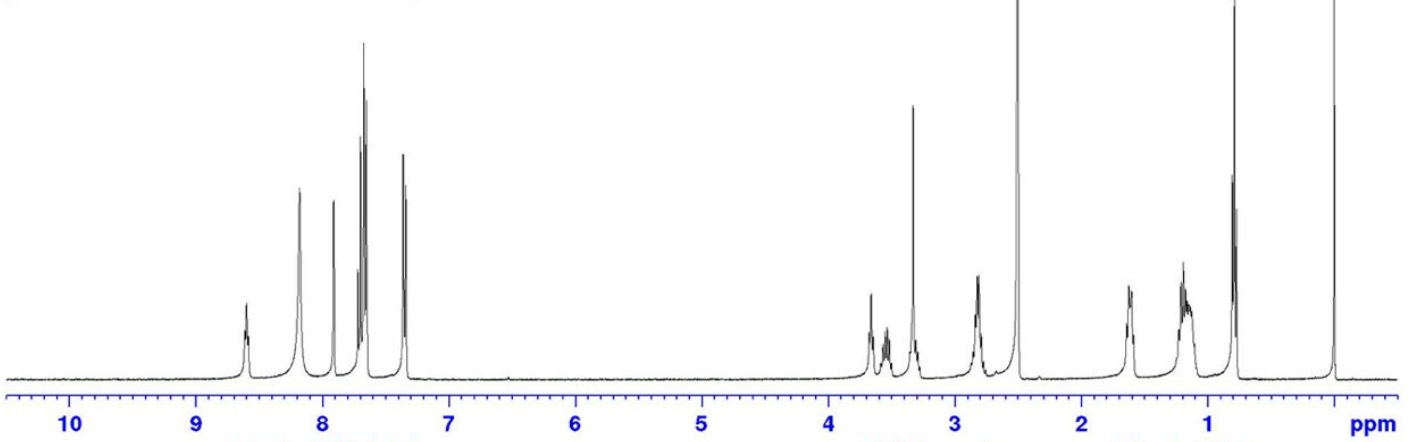

|

|ำ

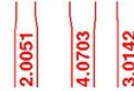
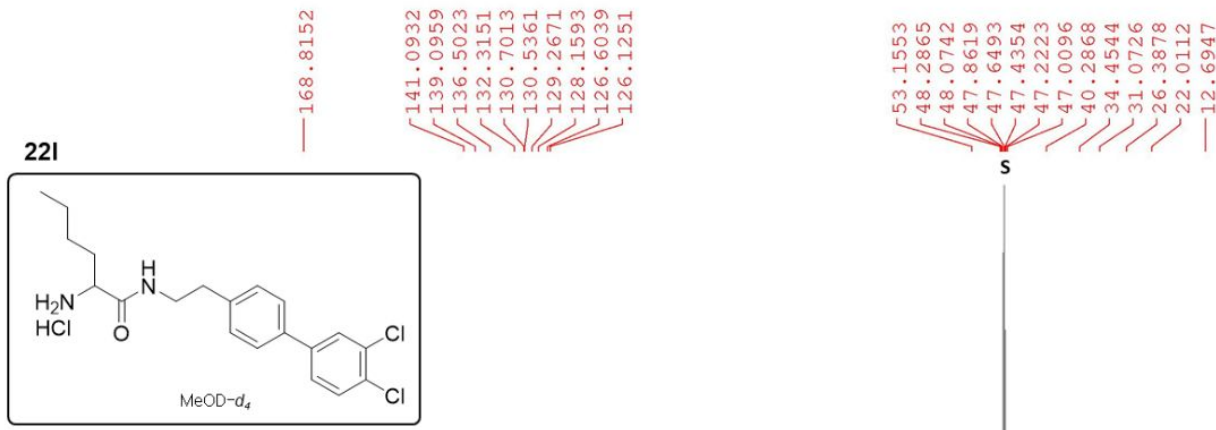

W: Water

S: Solvent

I: Impurity

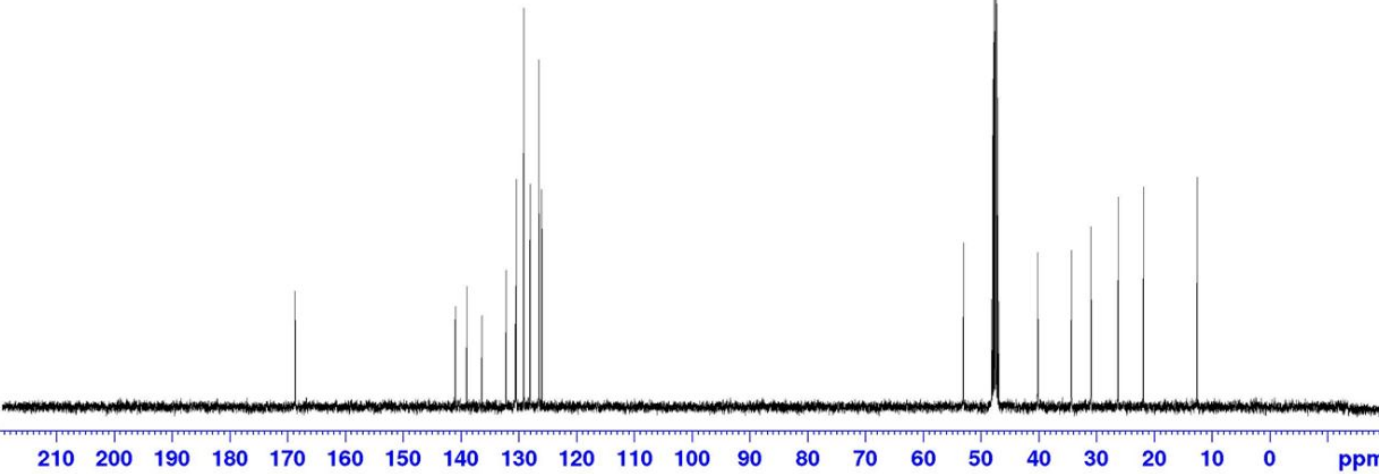



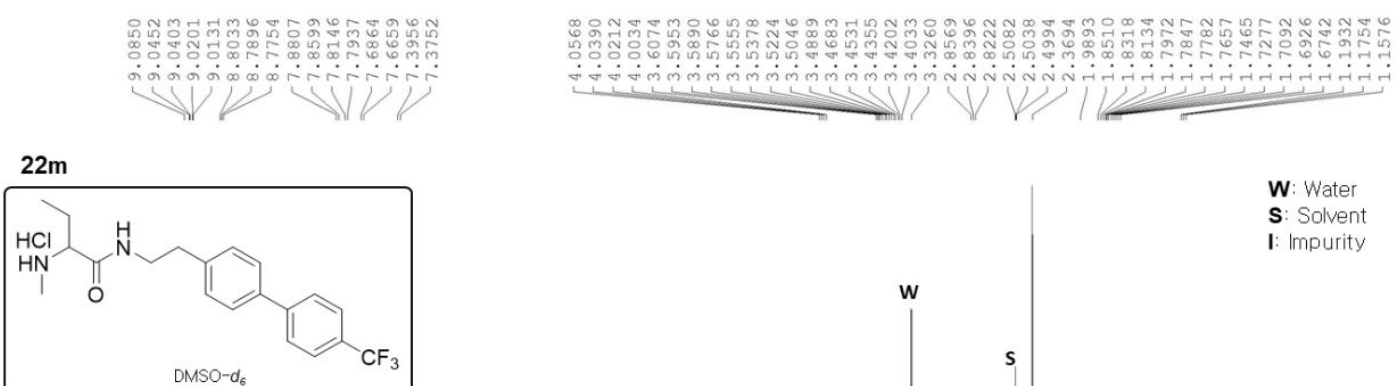

W: Water

S: Solvent
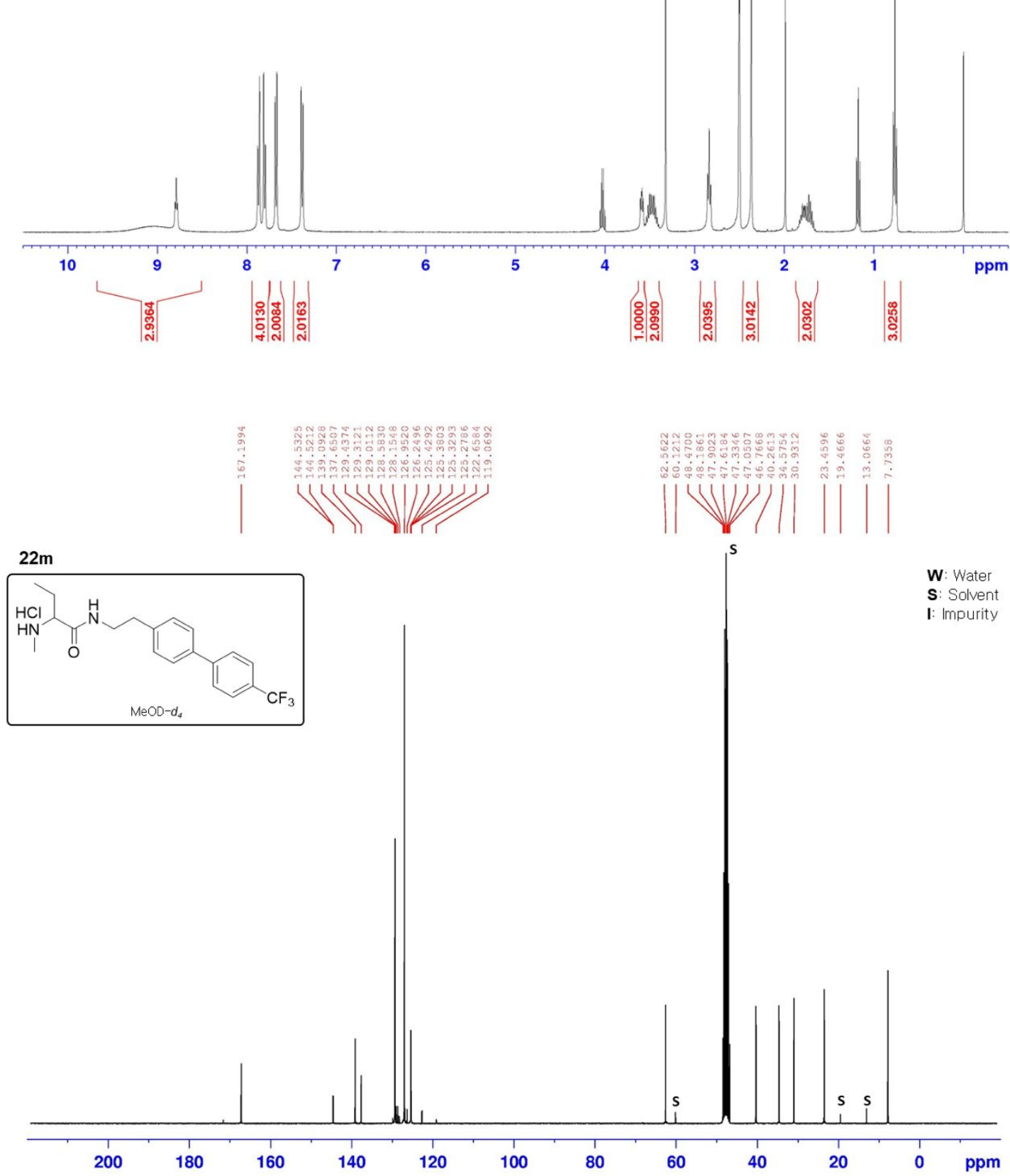

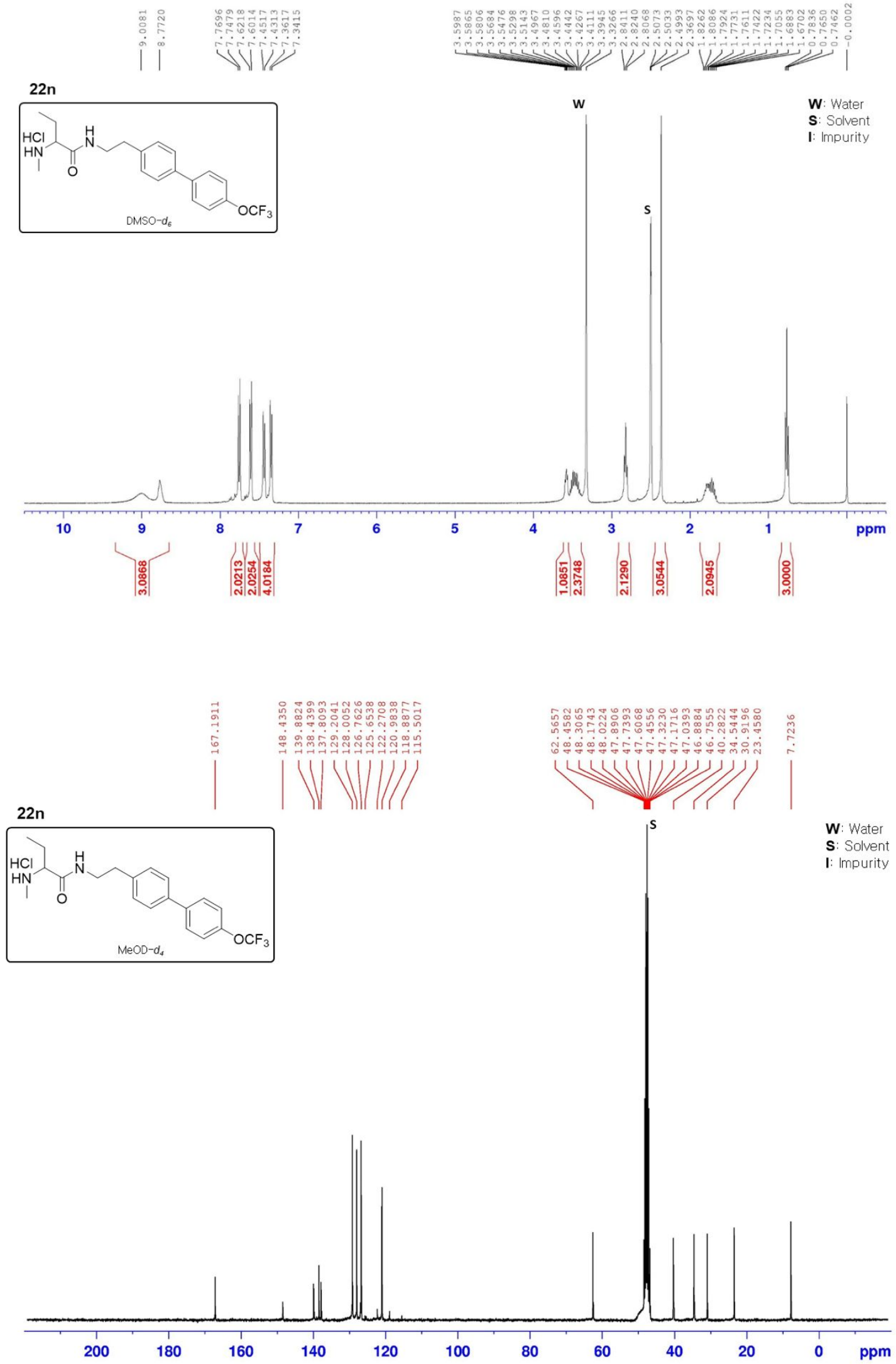


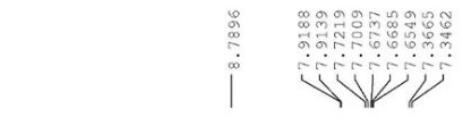

220
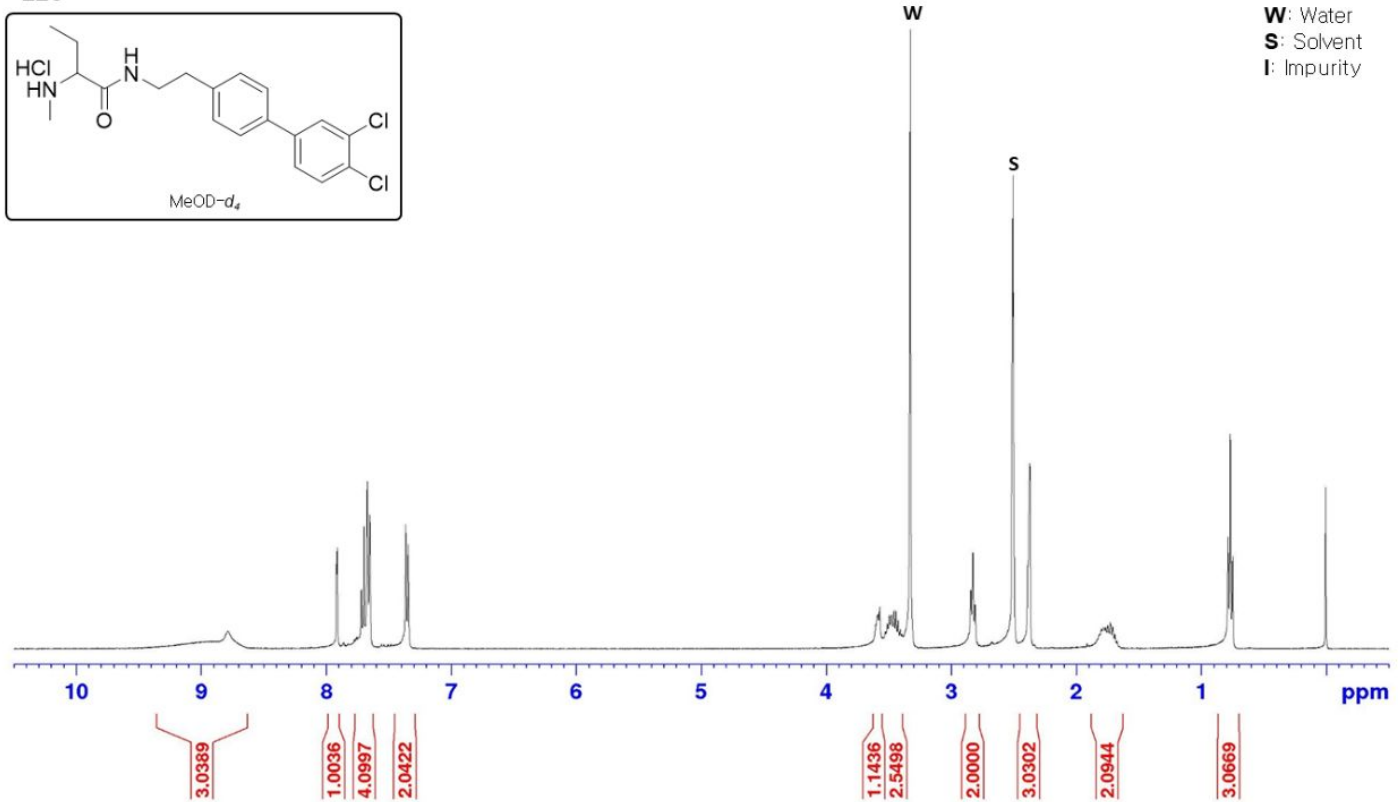

22o

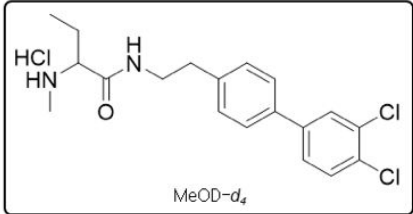

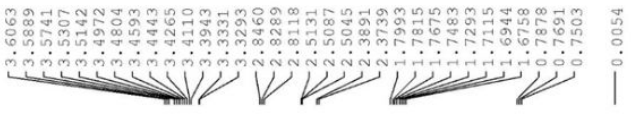

W: Water

S: Solvent

I: Impurity

w
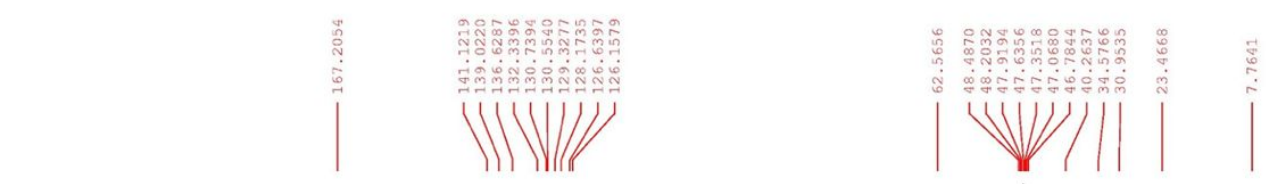

s

W: Water

S: Solvent

I: Impurity

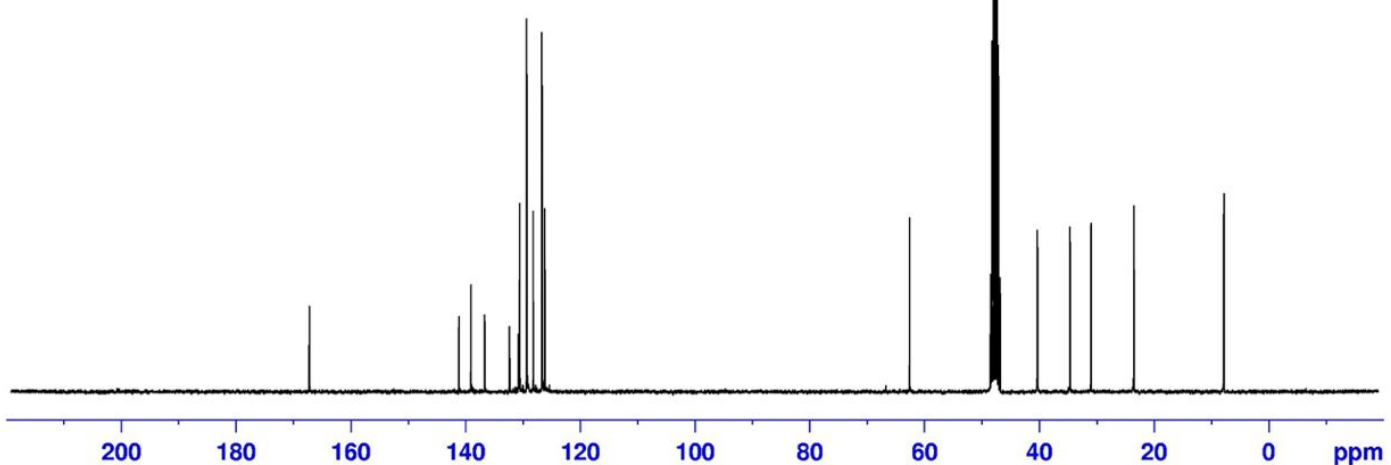



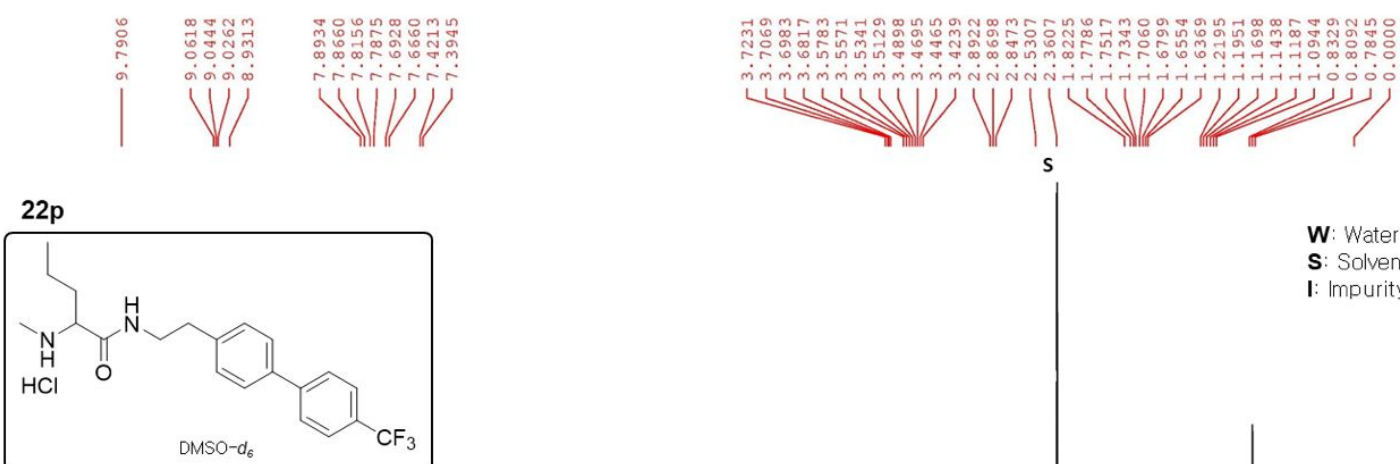

W: Water

S: Solvent

I: Impurity
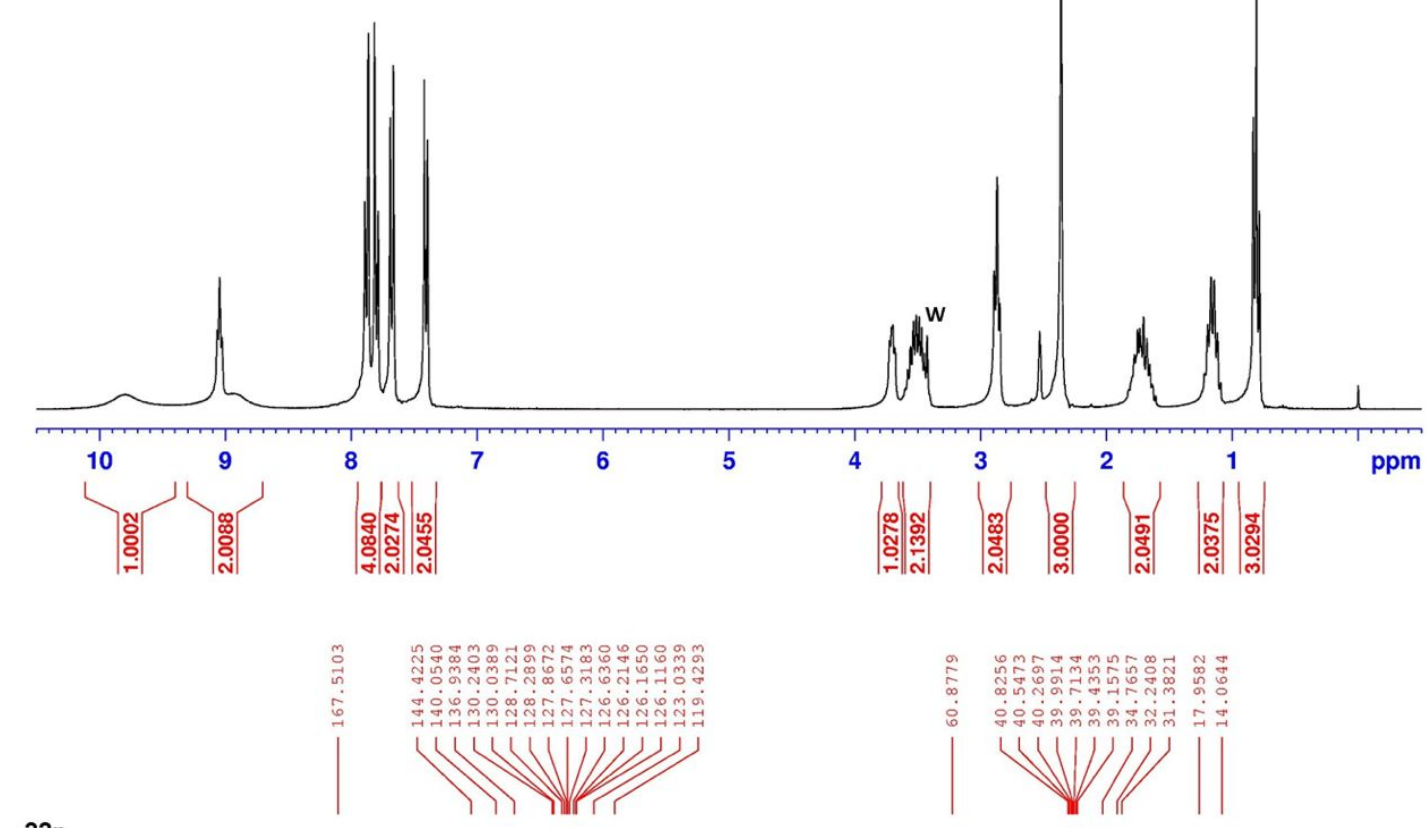

$22 p$

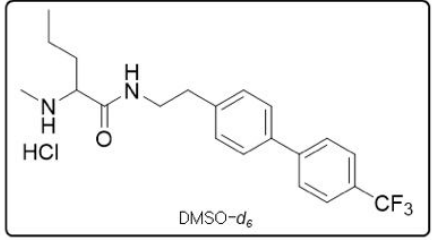

S: Solvent

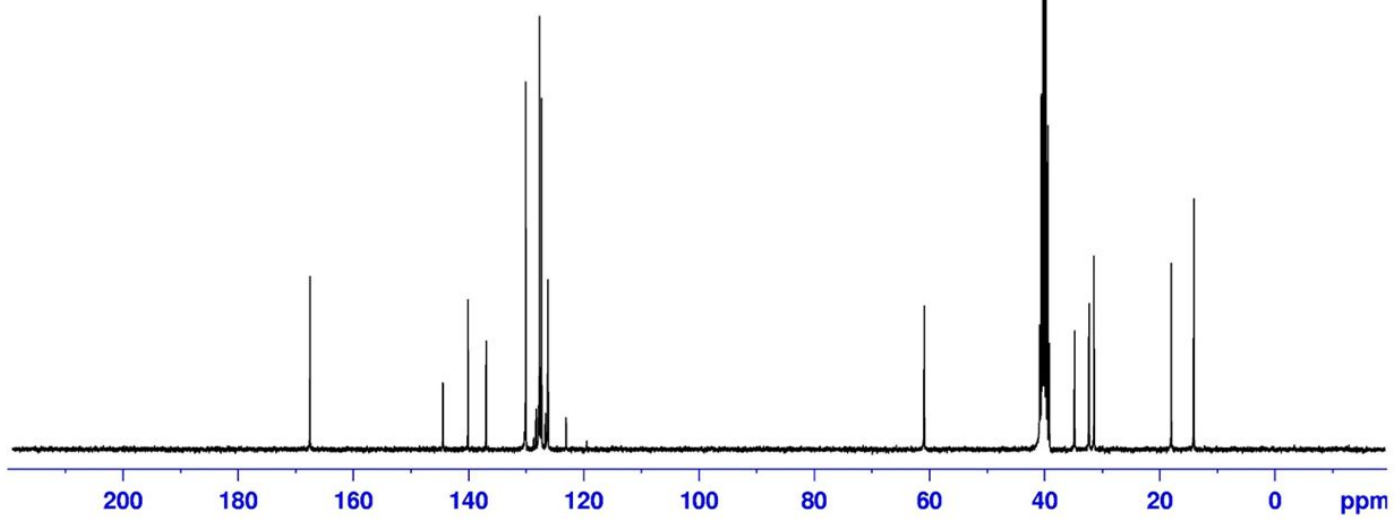




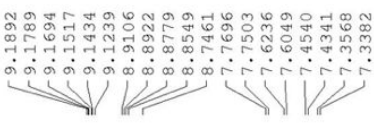

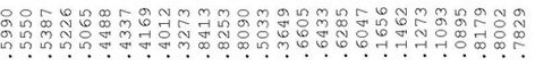

$22 q$
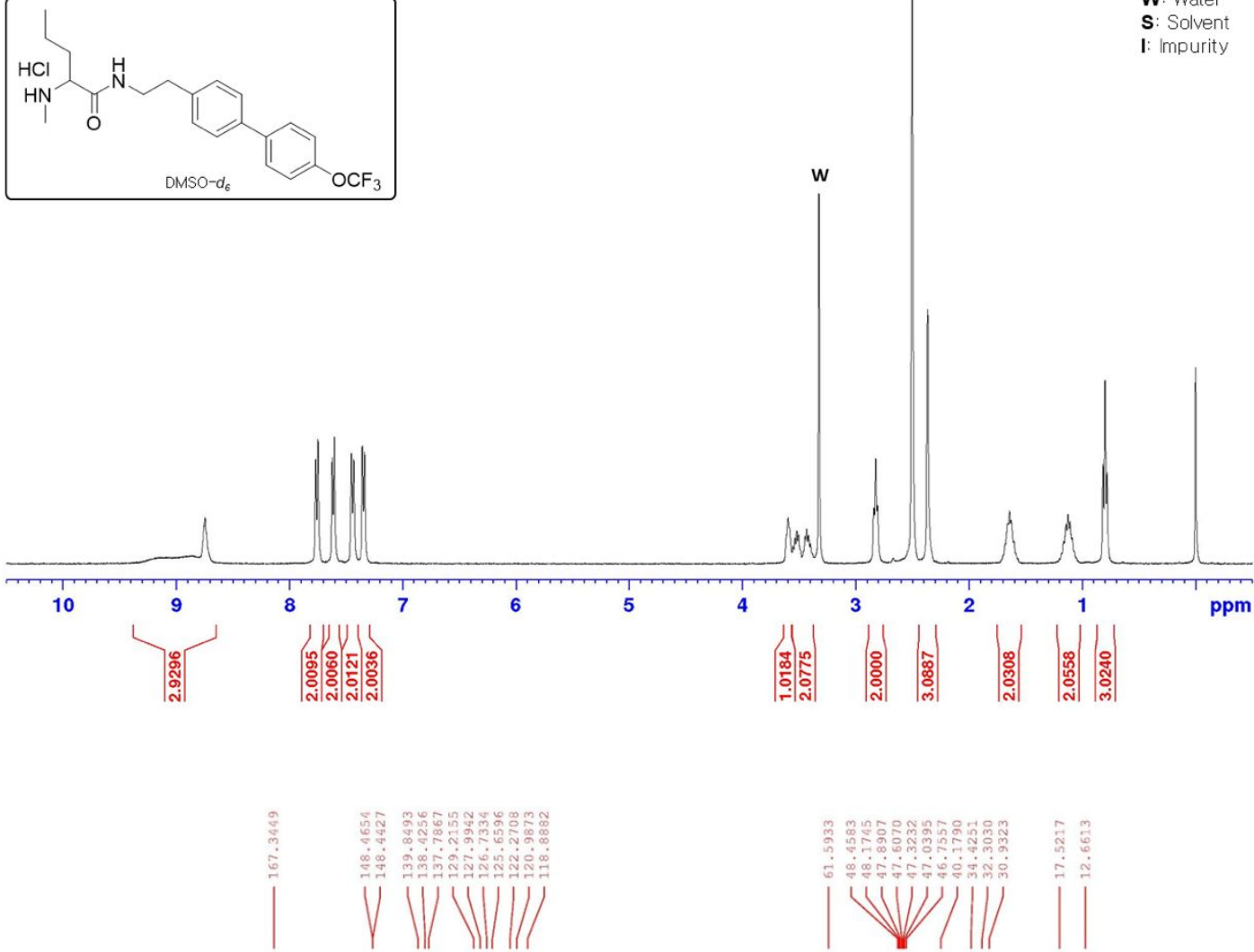

$22 q$

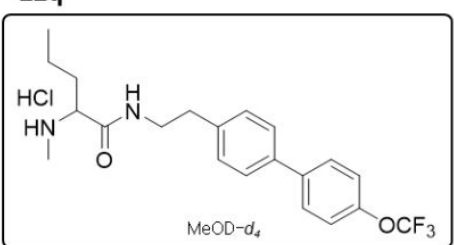

s

W: Water

S: Solvent

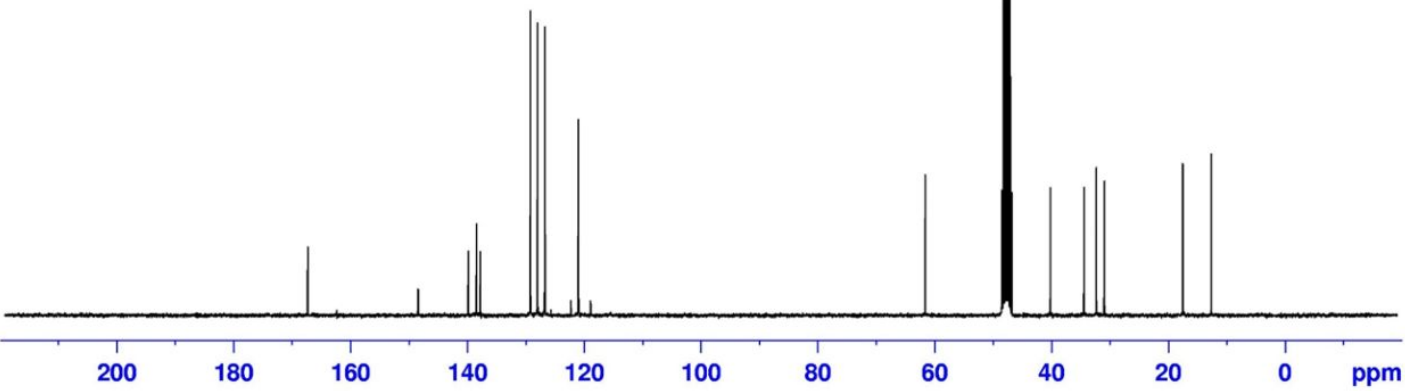



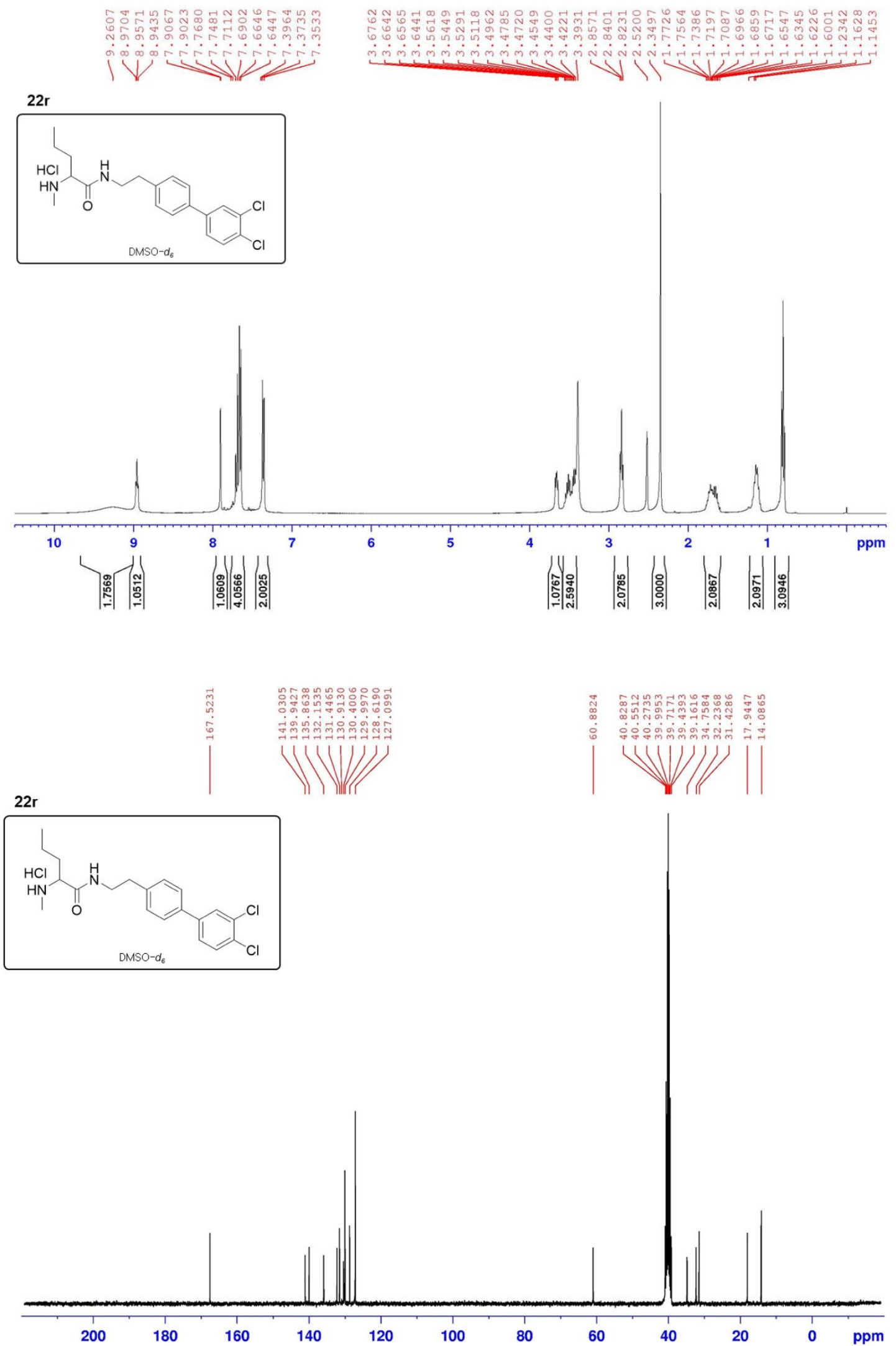
22s
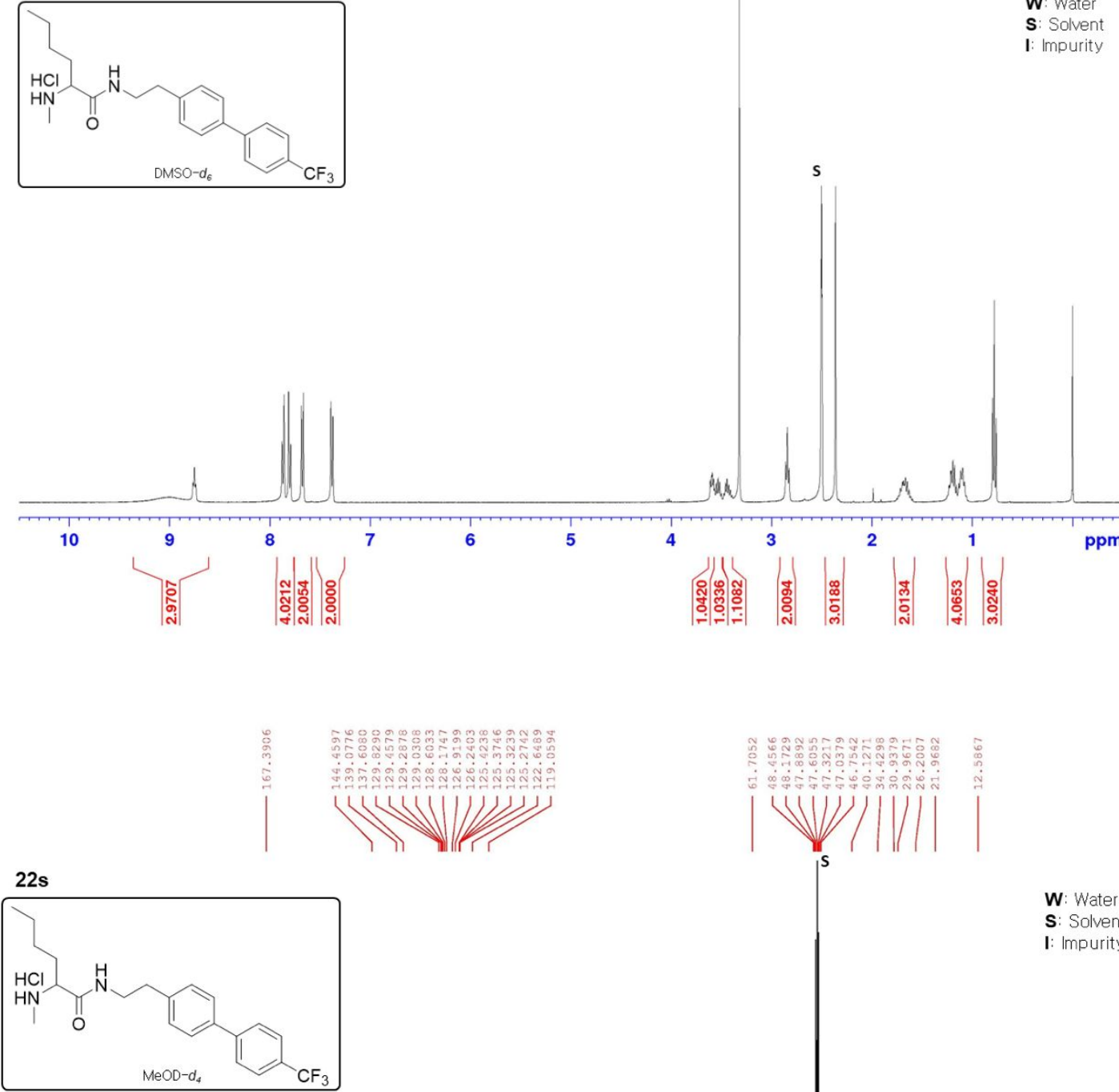

w

I: Impurity
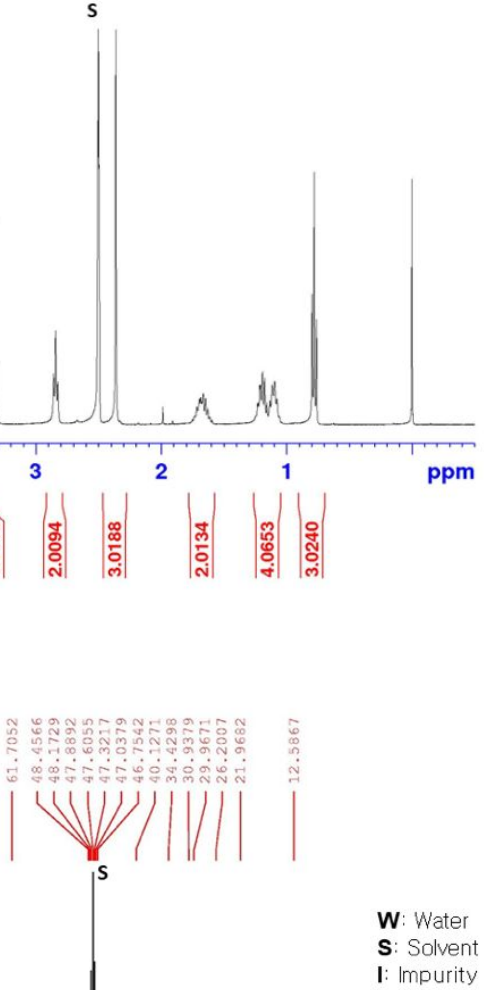

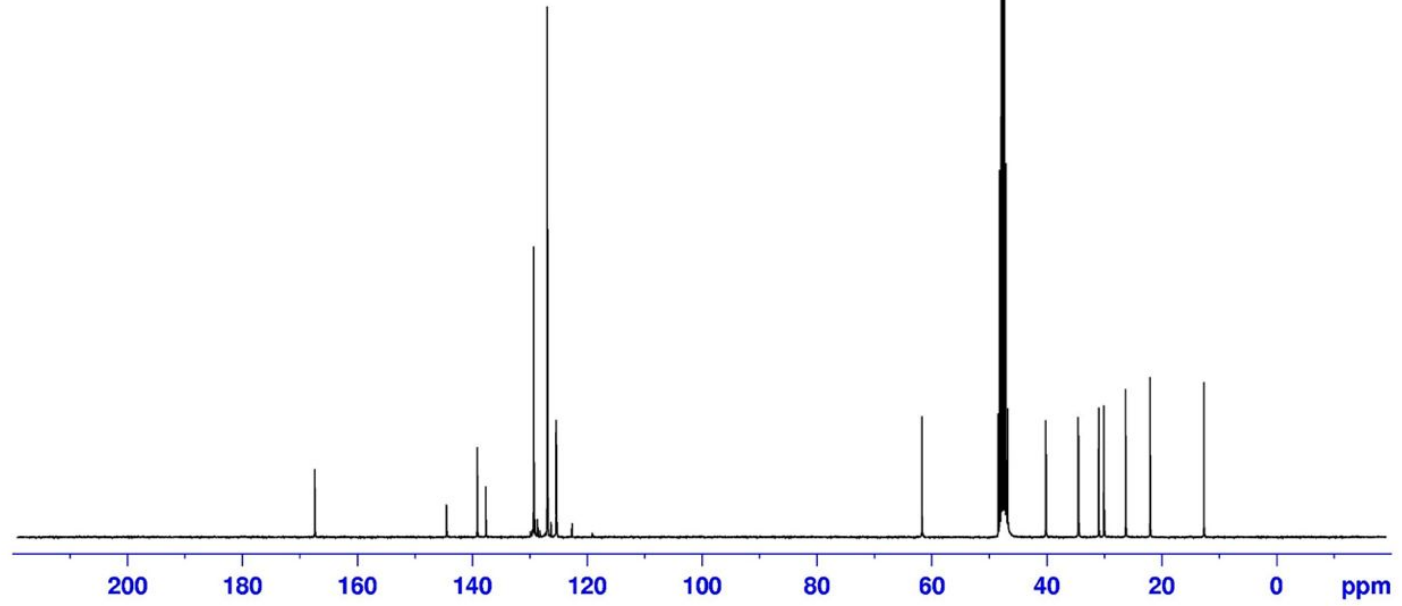



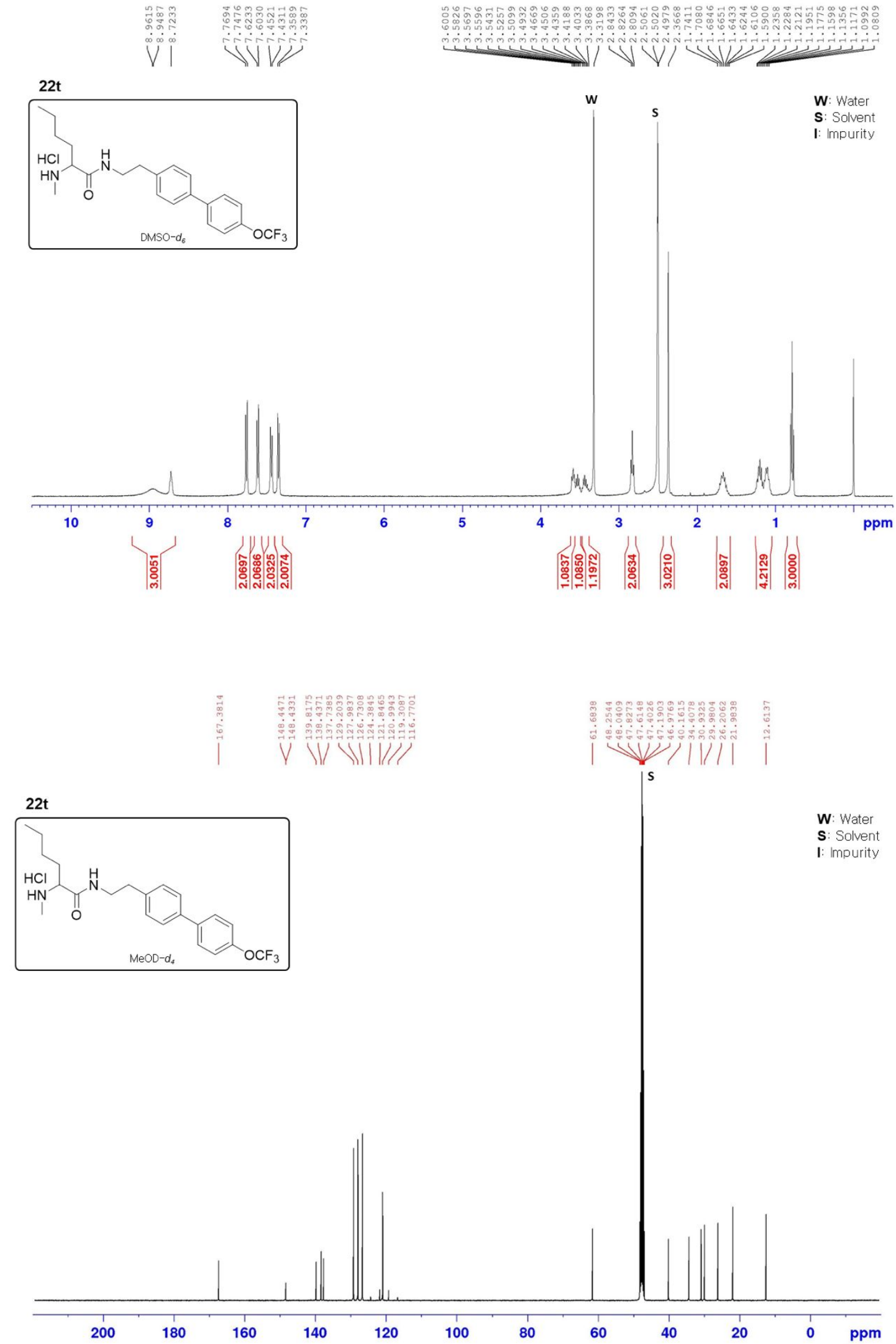

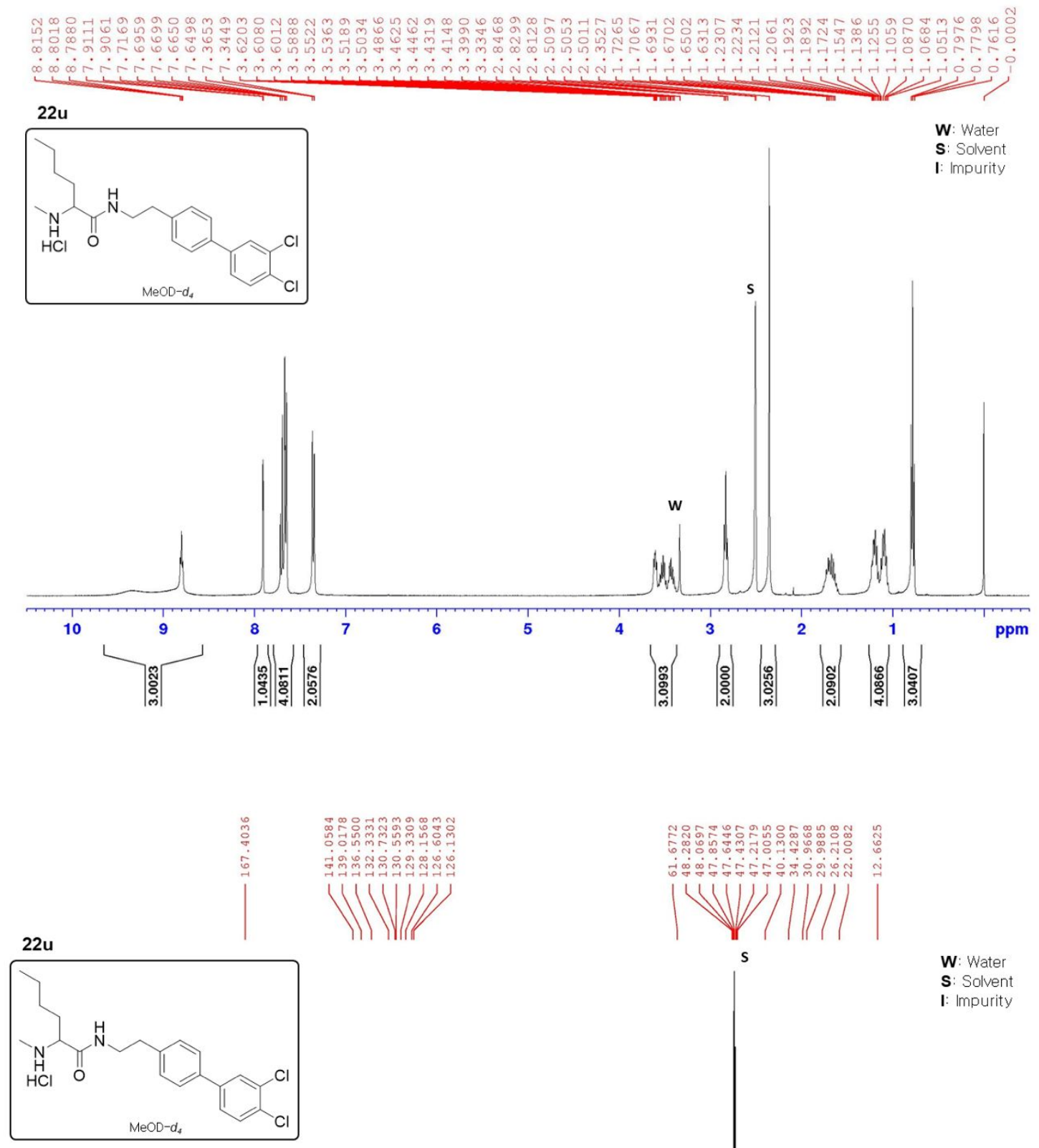

W: Water

S: Solvent

I: Impurity

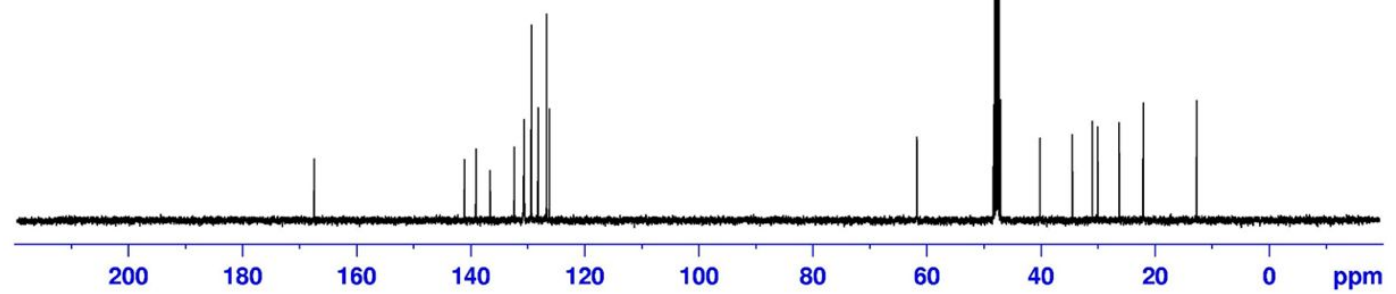



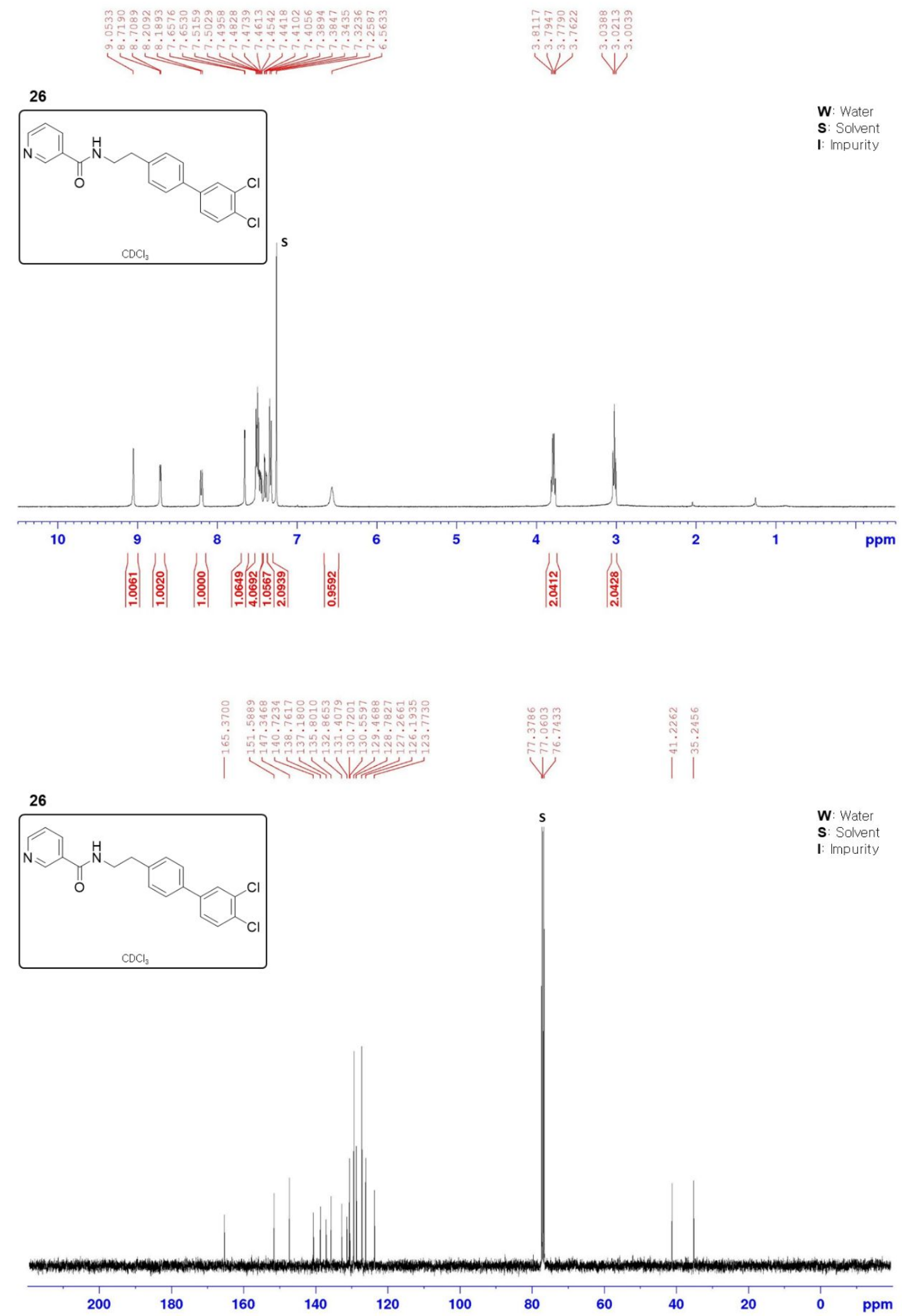

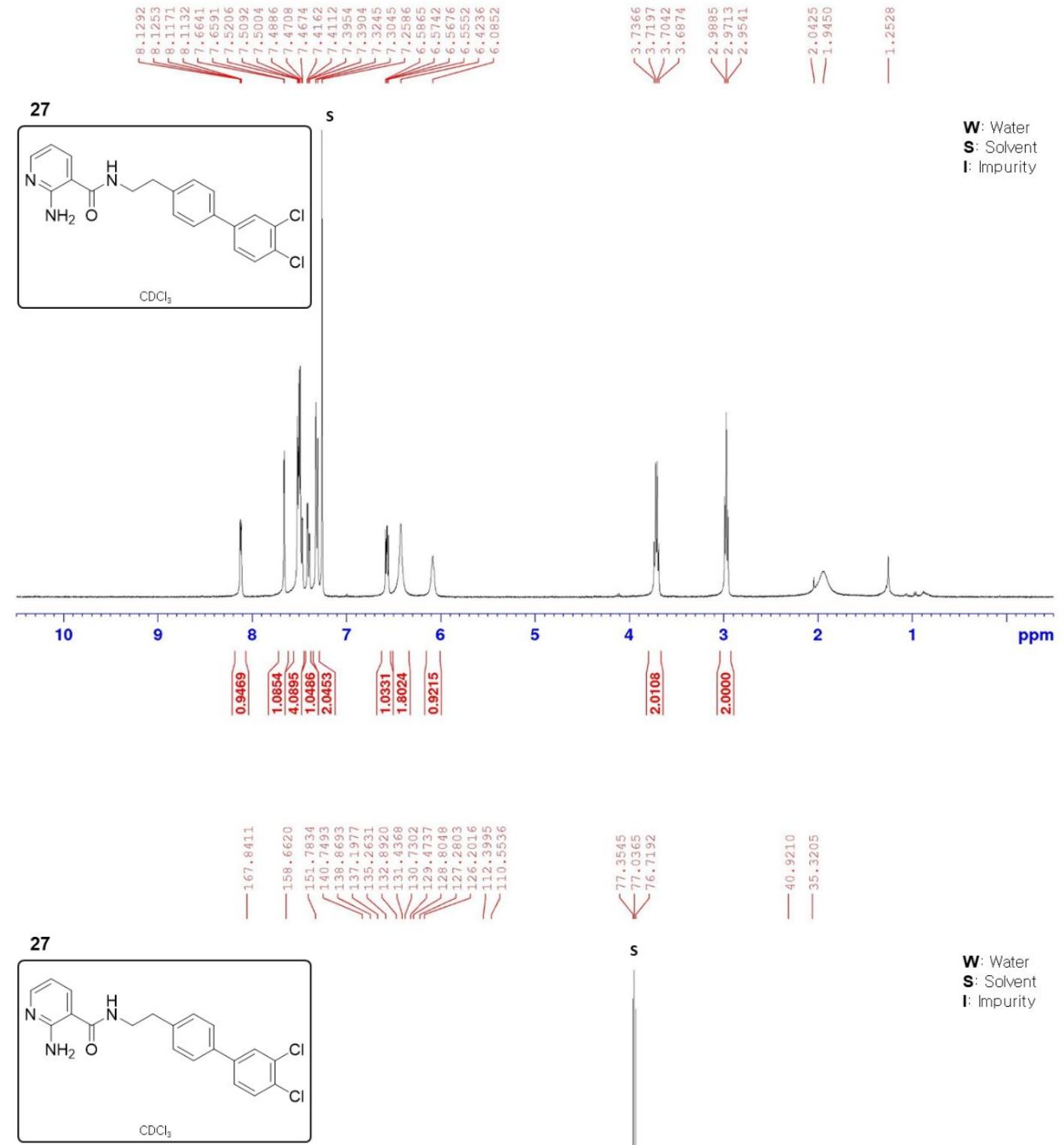

W: Water

S: Solvent

I: Impurity

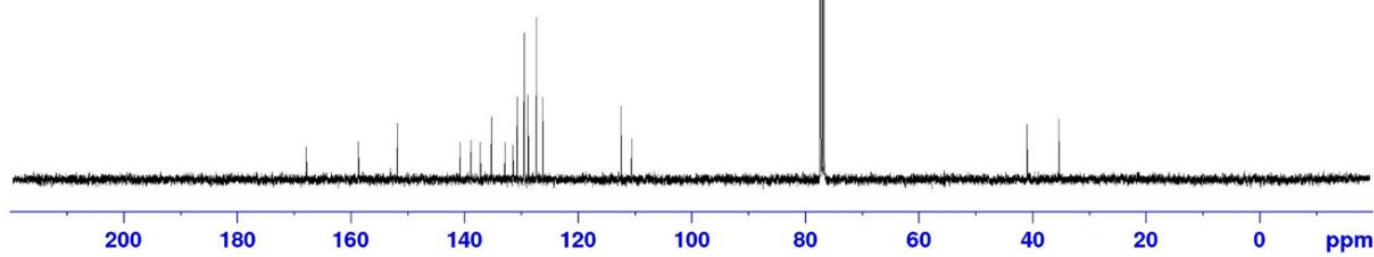



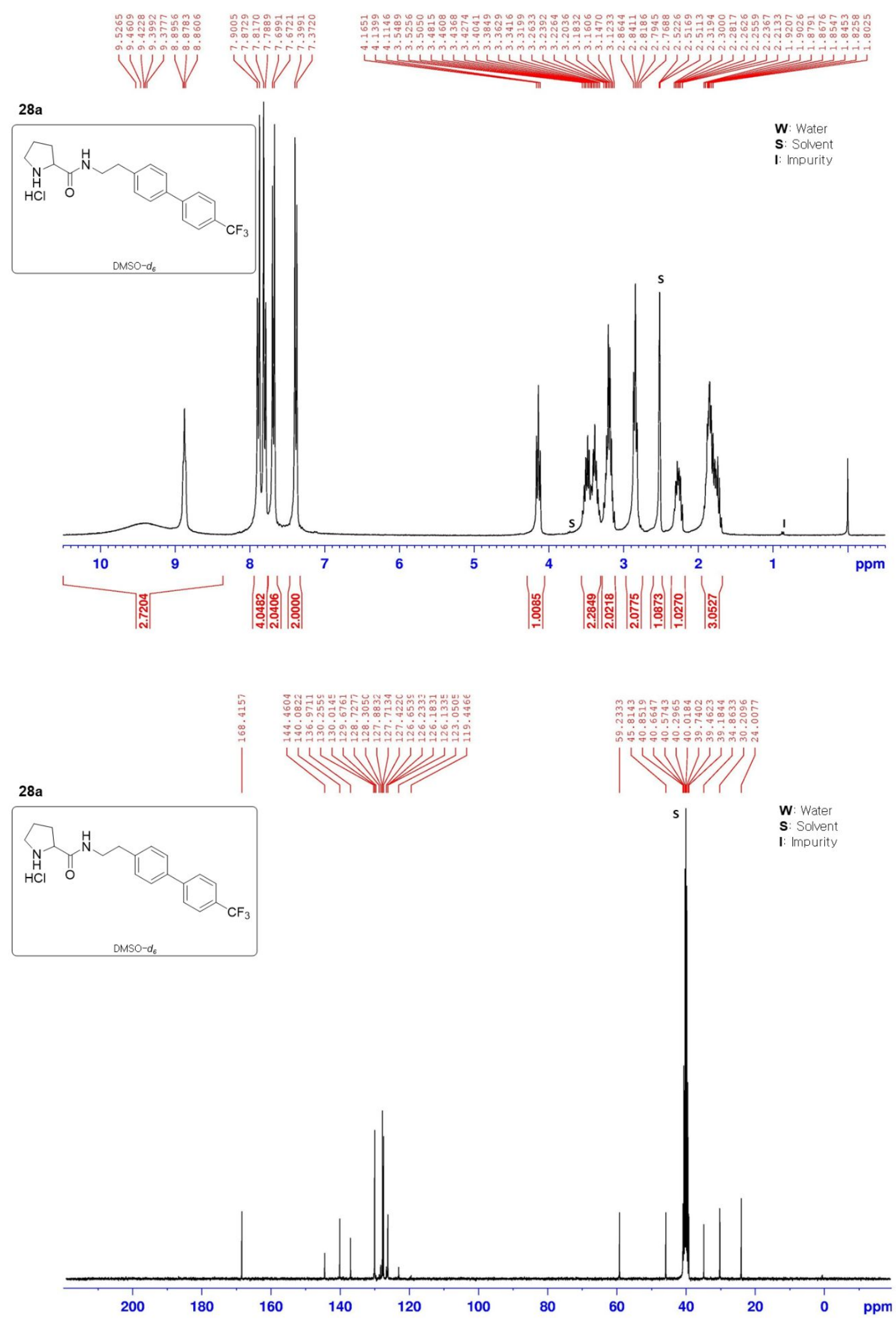

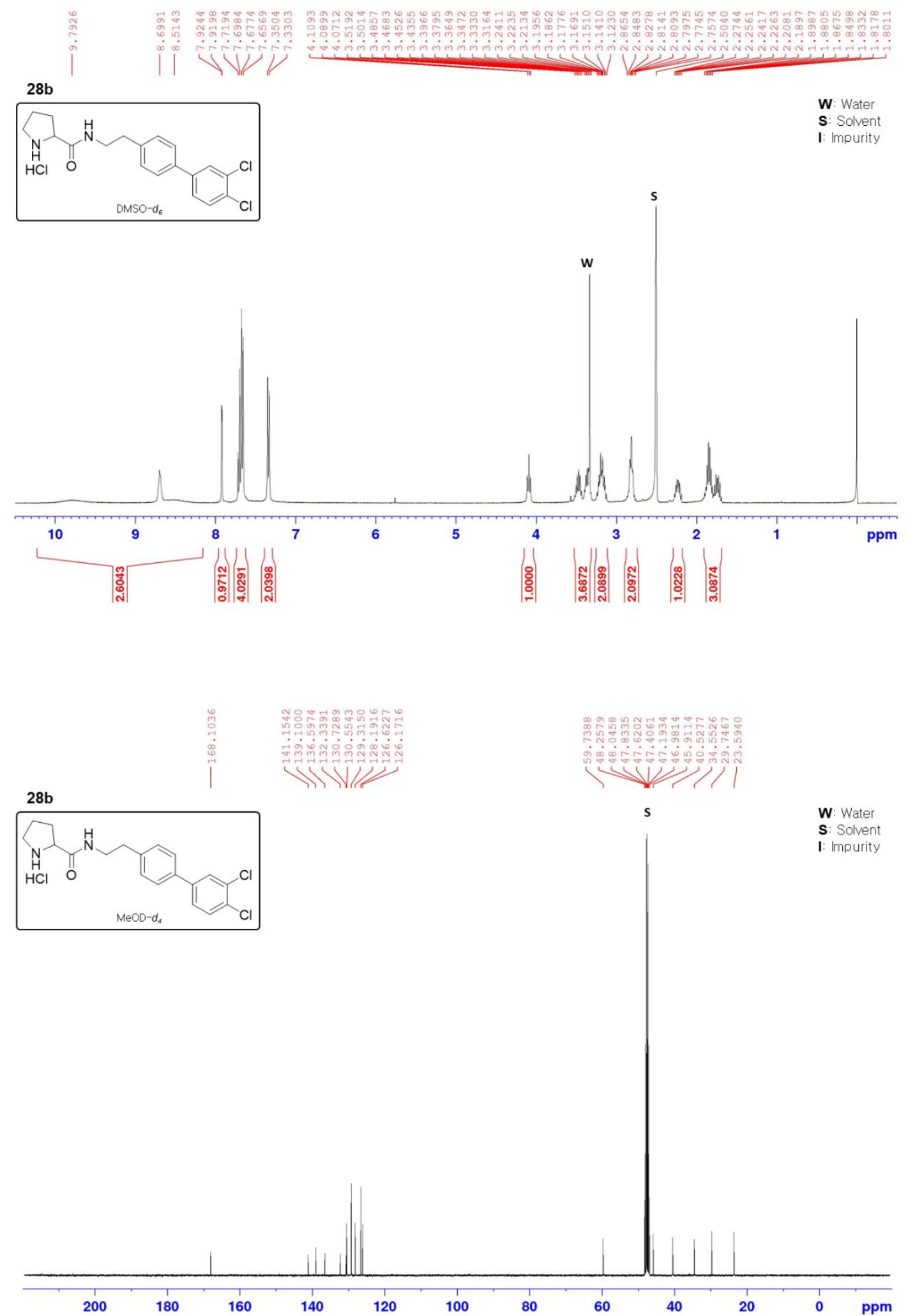

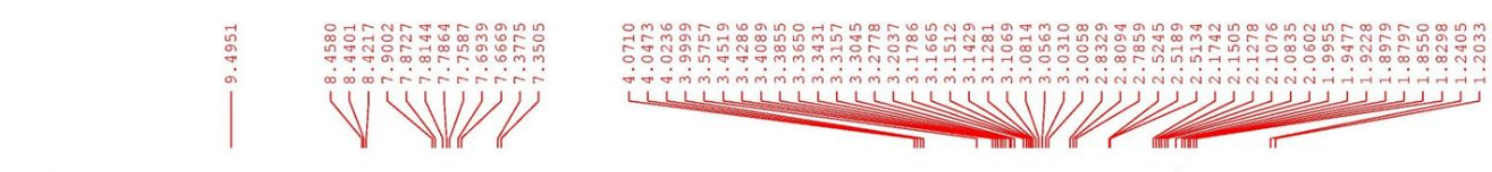

$29 a$
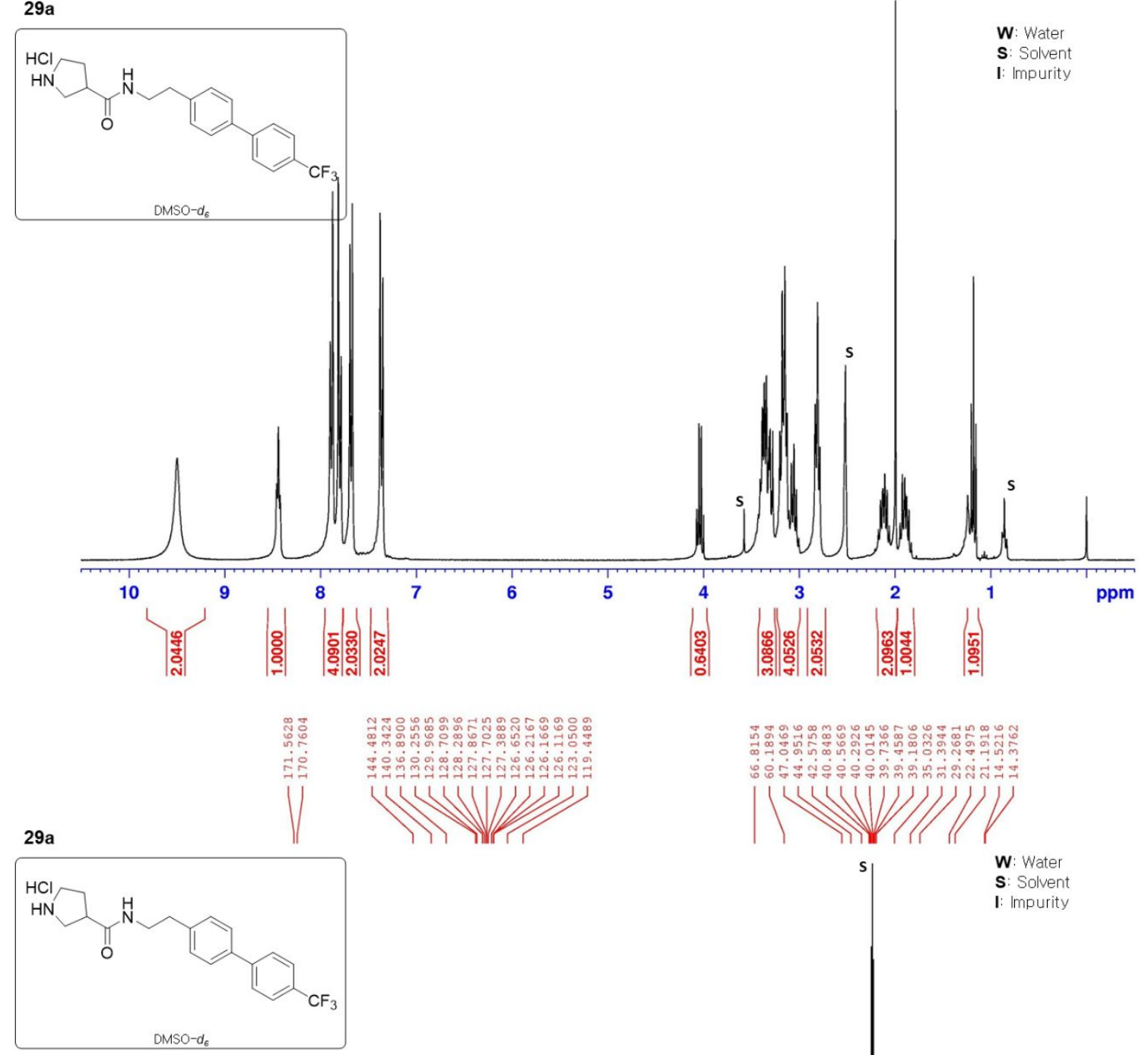

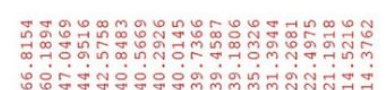
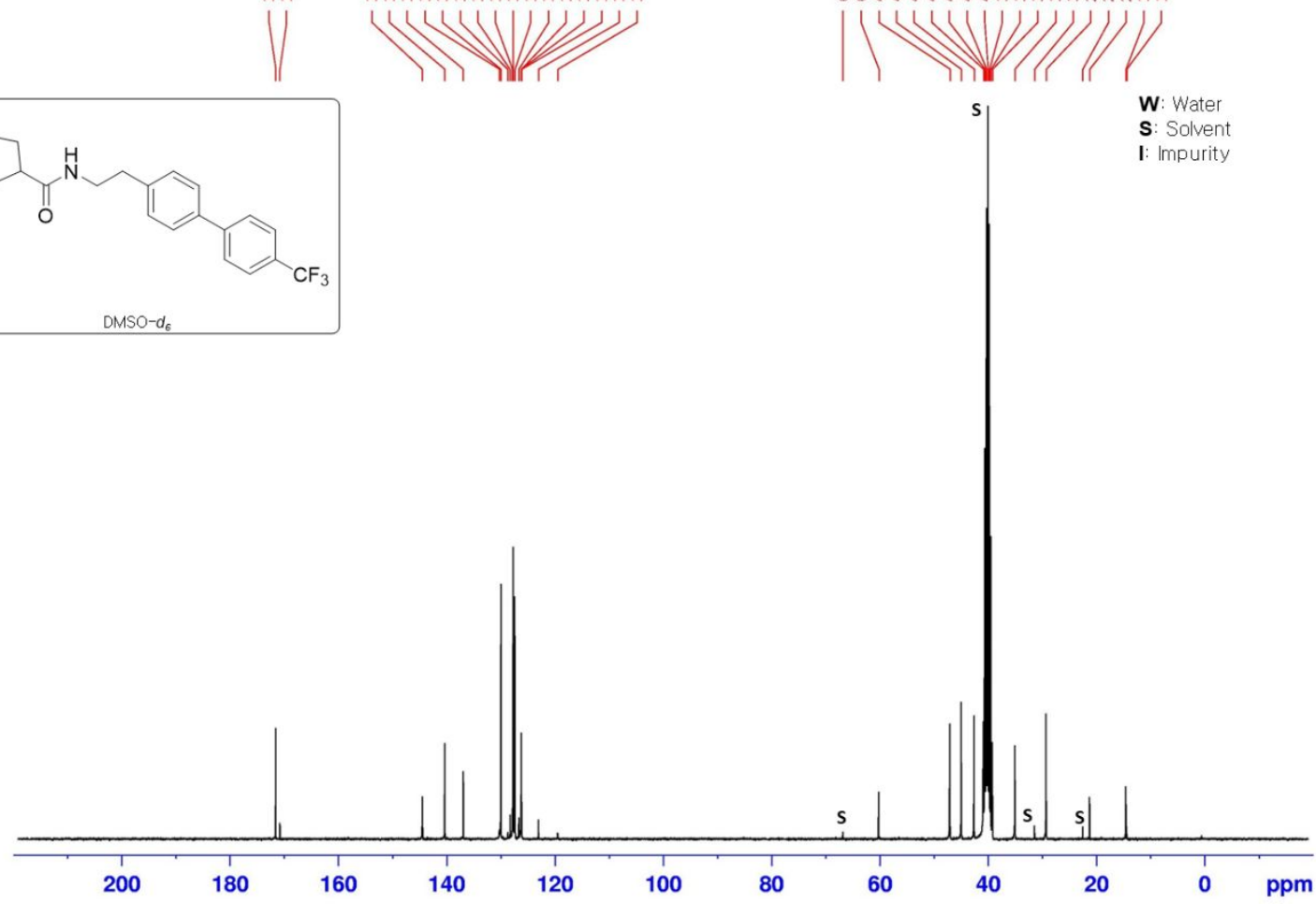

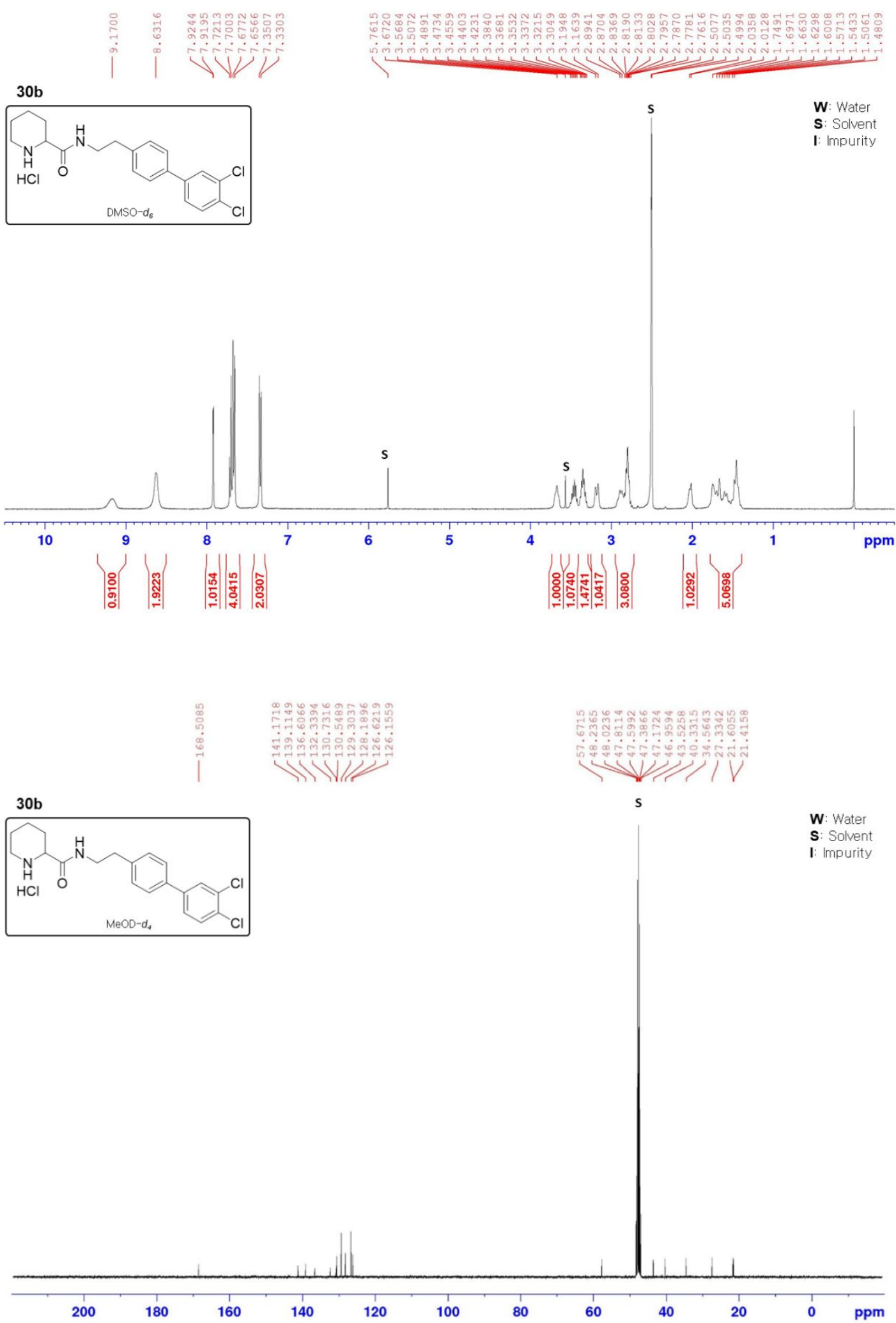

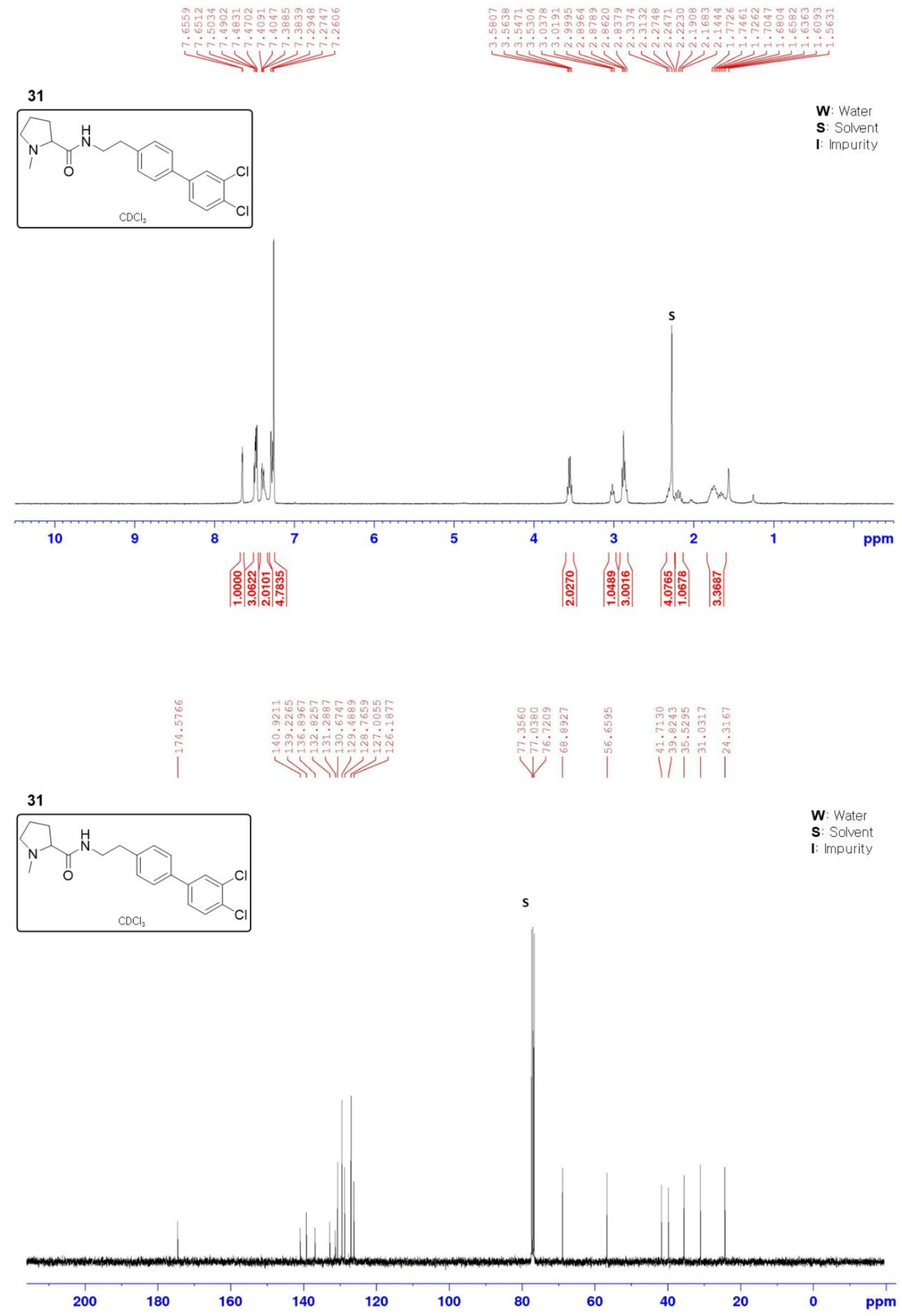

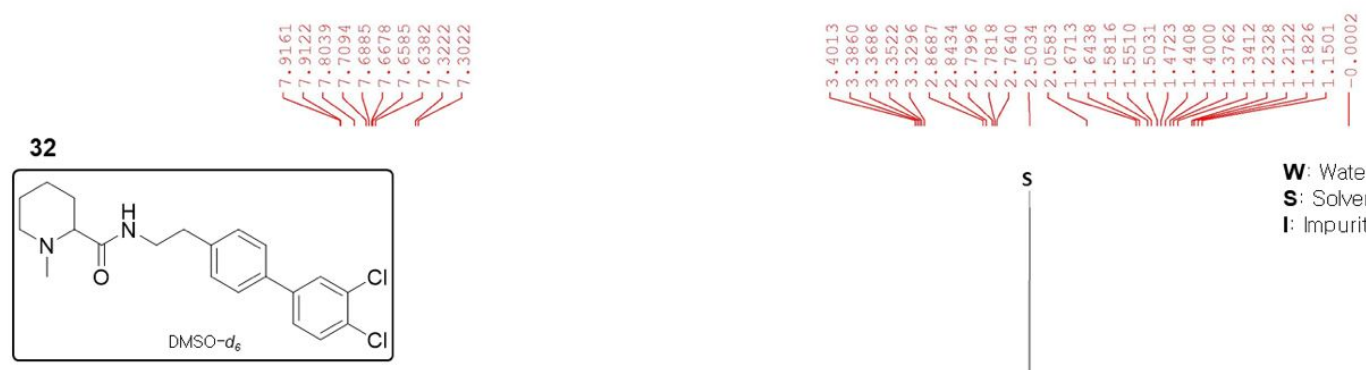

W: Water

S: Solvent

I: Impurity
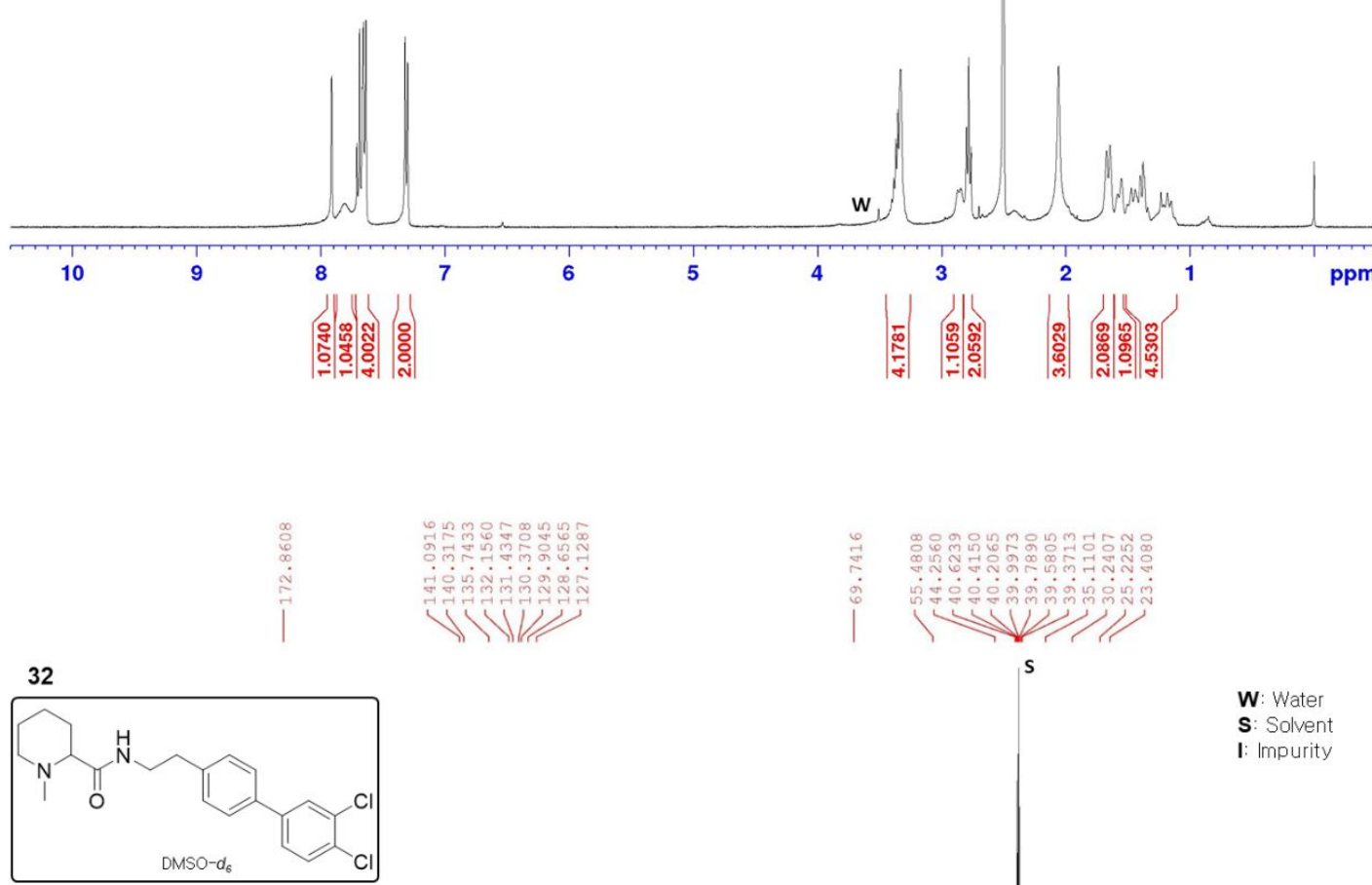

S: Solvent

I: Impurity

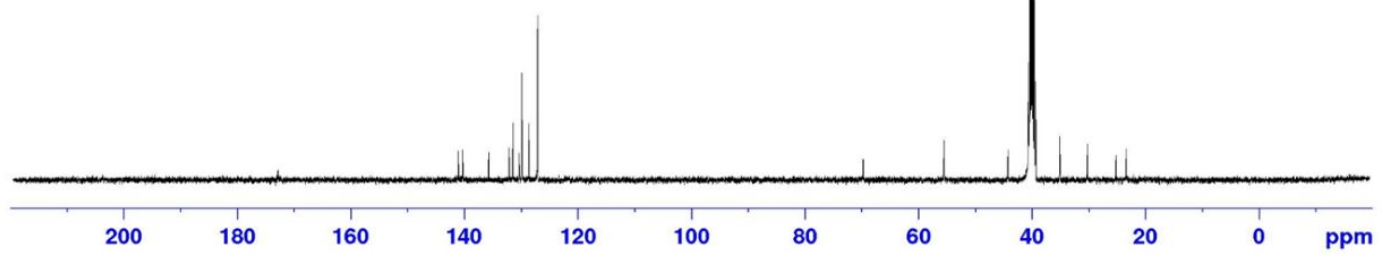




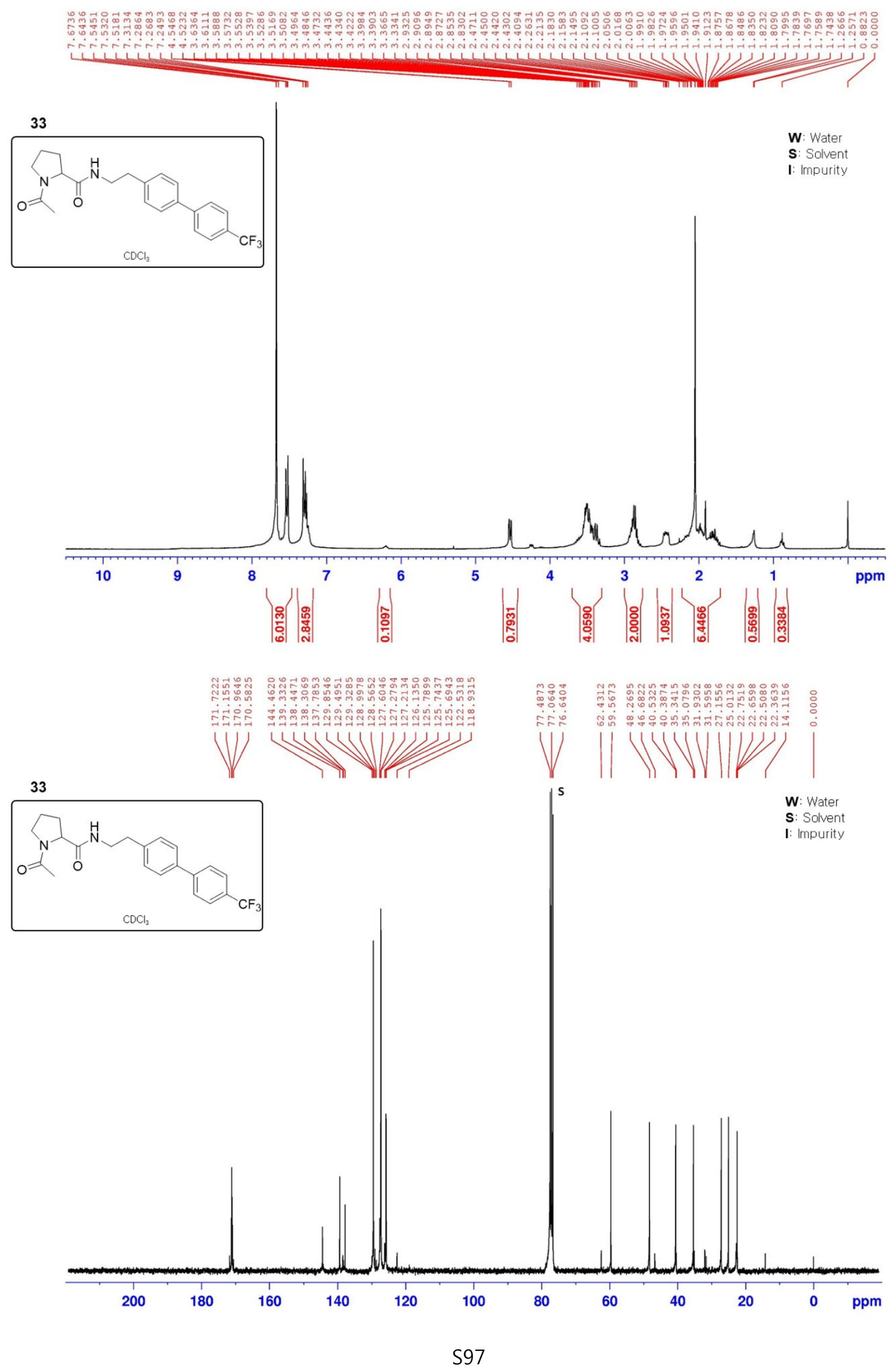



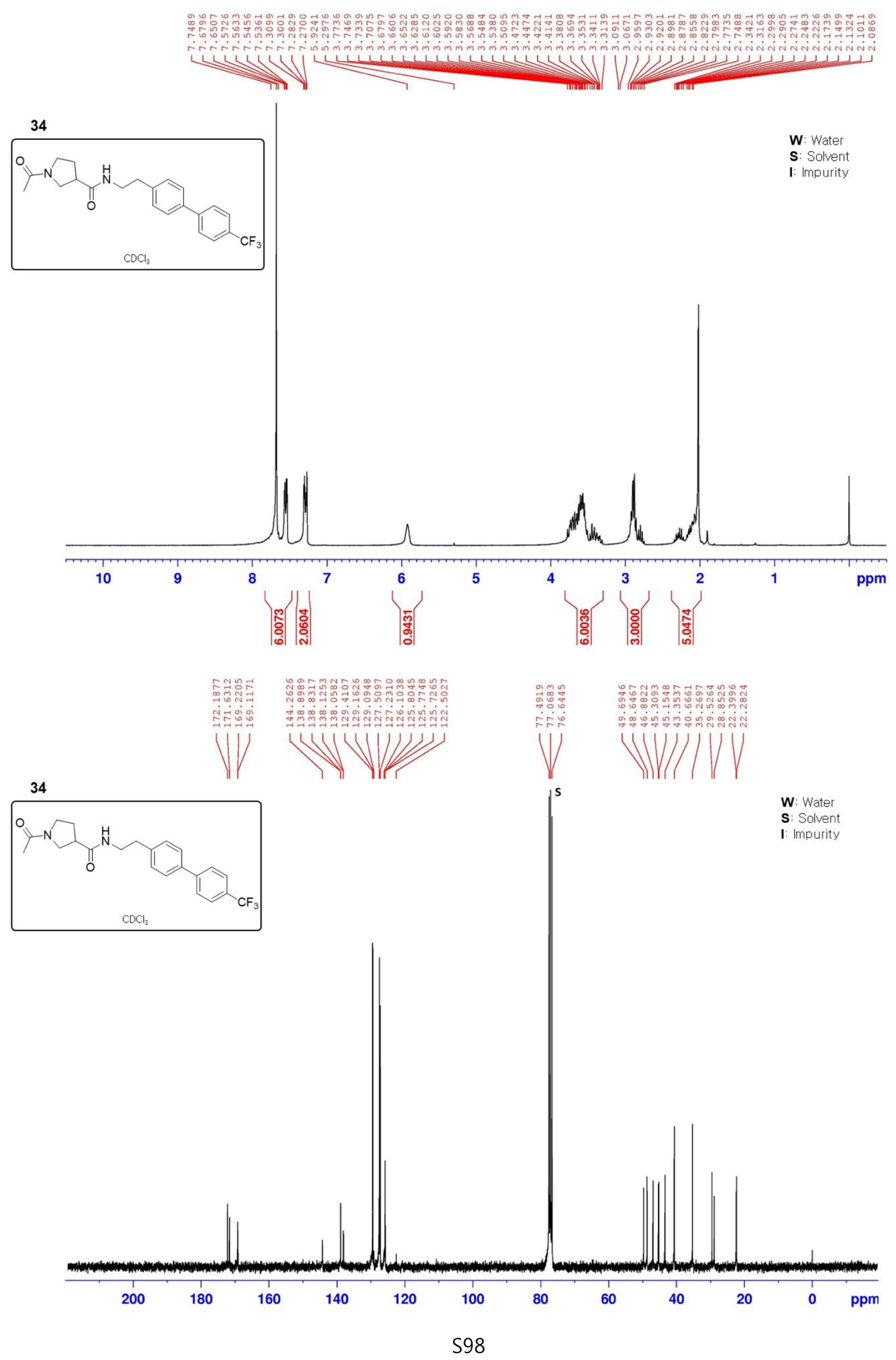


\section{HRMS analysis}

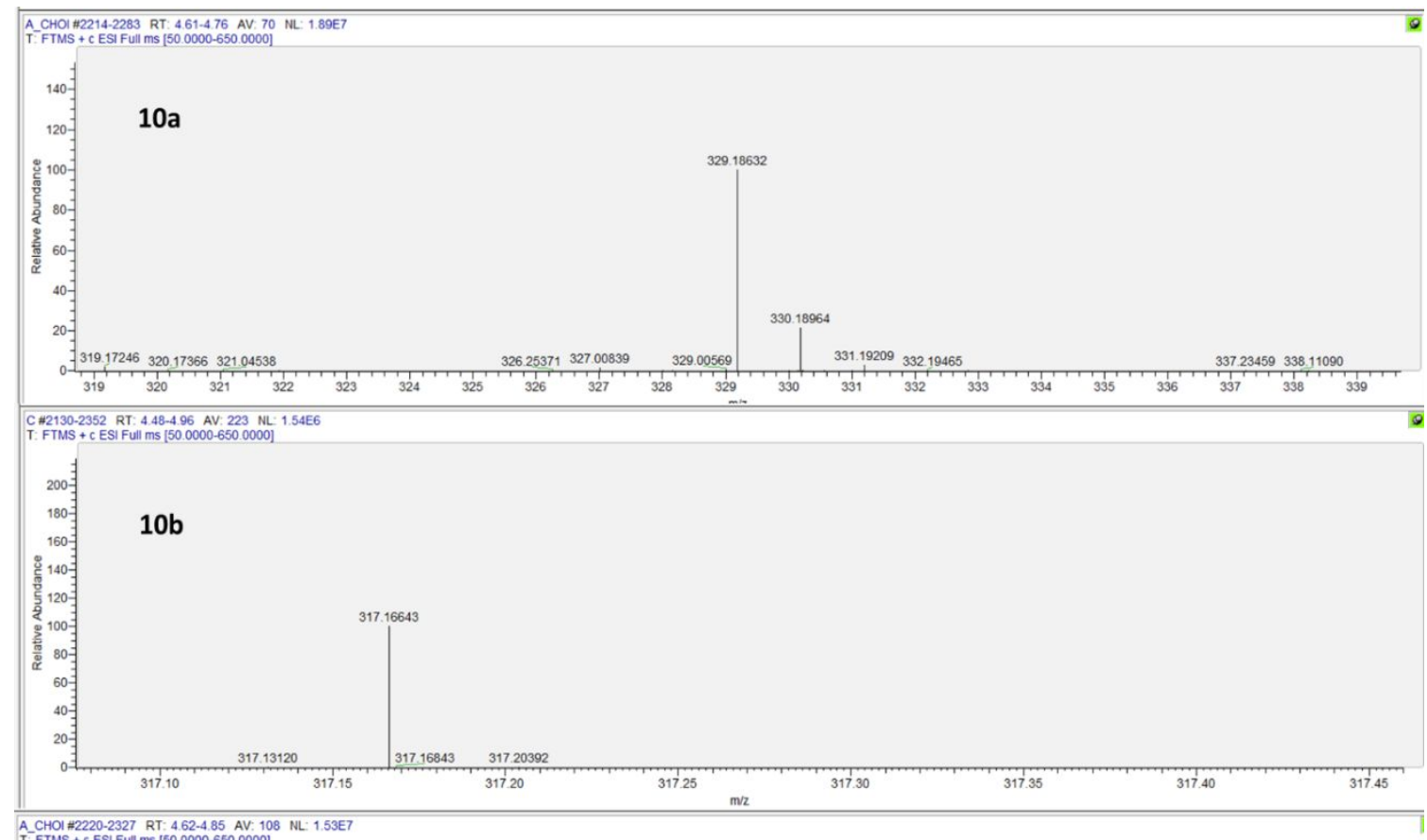

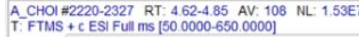

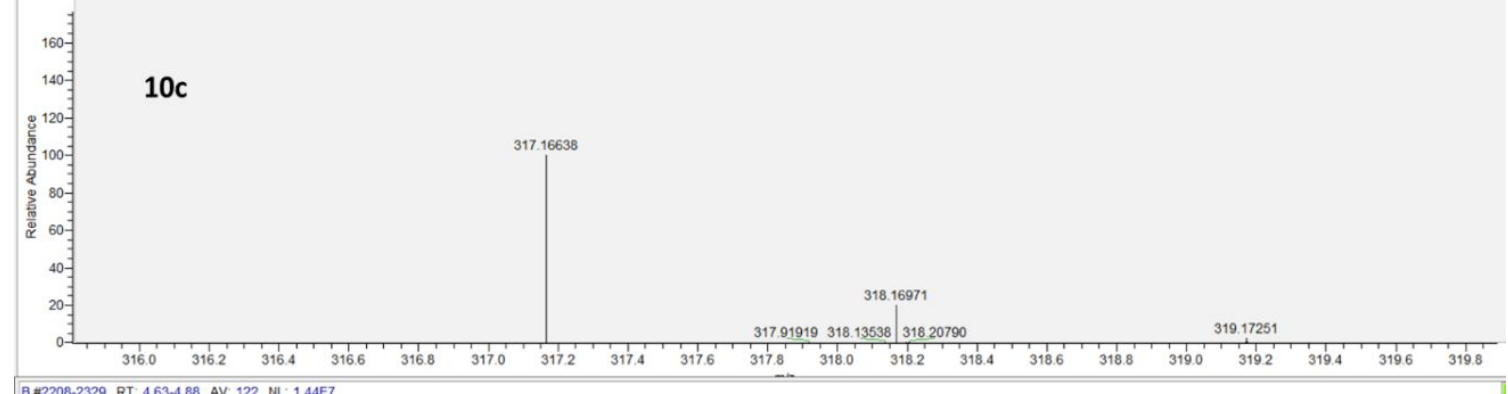
\begin{tabular}{l}
\hline B $12208-2329$ RT: $4.63-4.88$ AV: 122 NL: 1.44E7 \\
T: FTMS + E ESI Full ms [50.0000-650.0000]
\end{tabular}

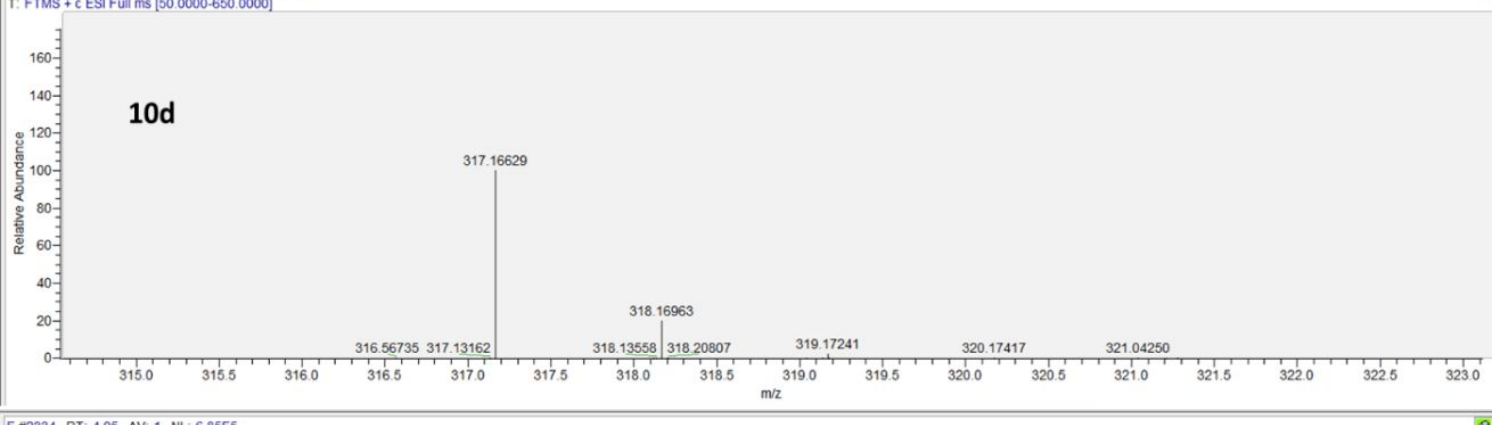

F 22334 RT: 4.95 AV: 1 NL- $6.85 E 5$
T: FTMS + ESI Full mS 500.000650 .0000$]$

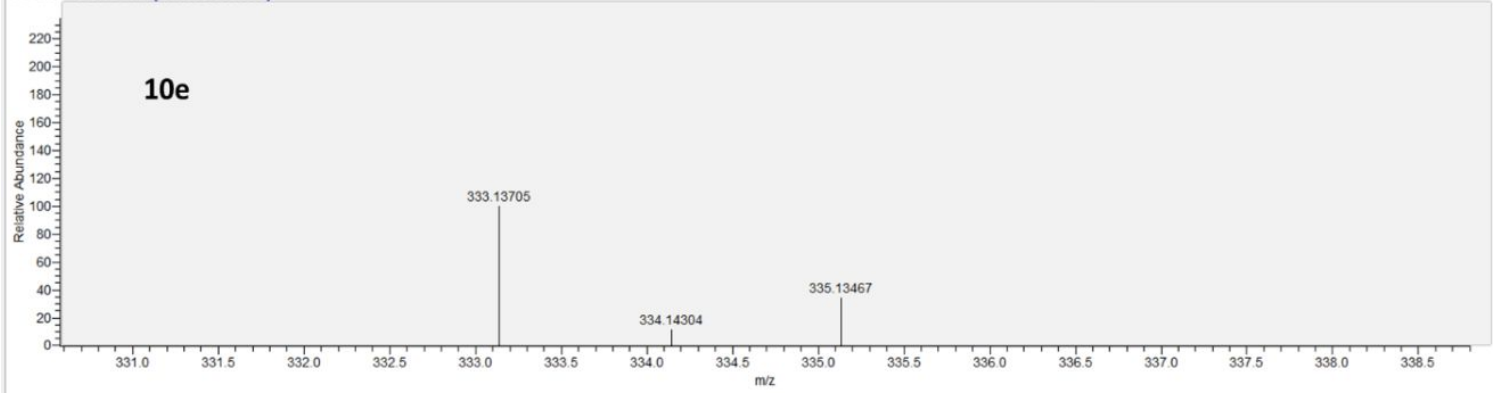




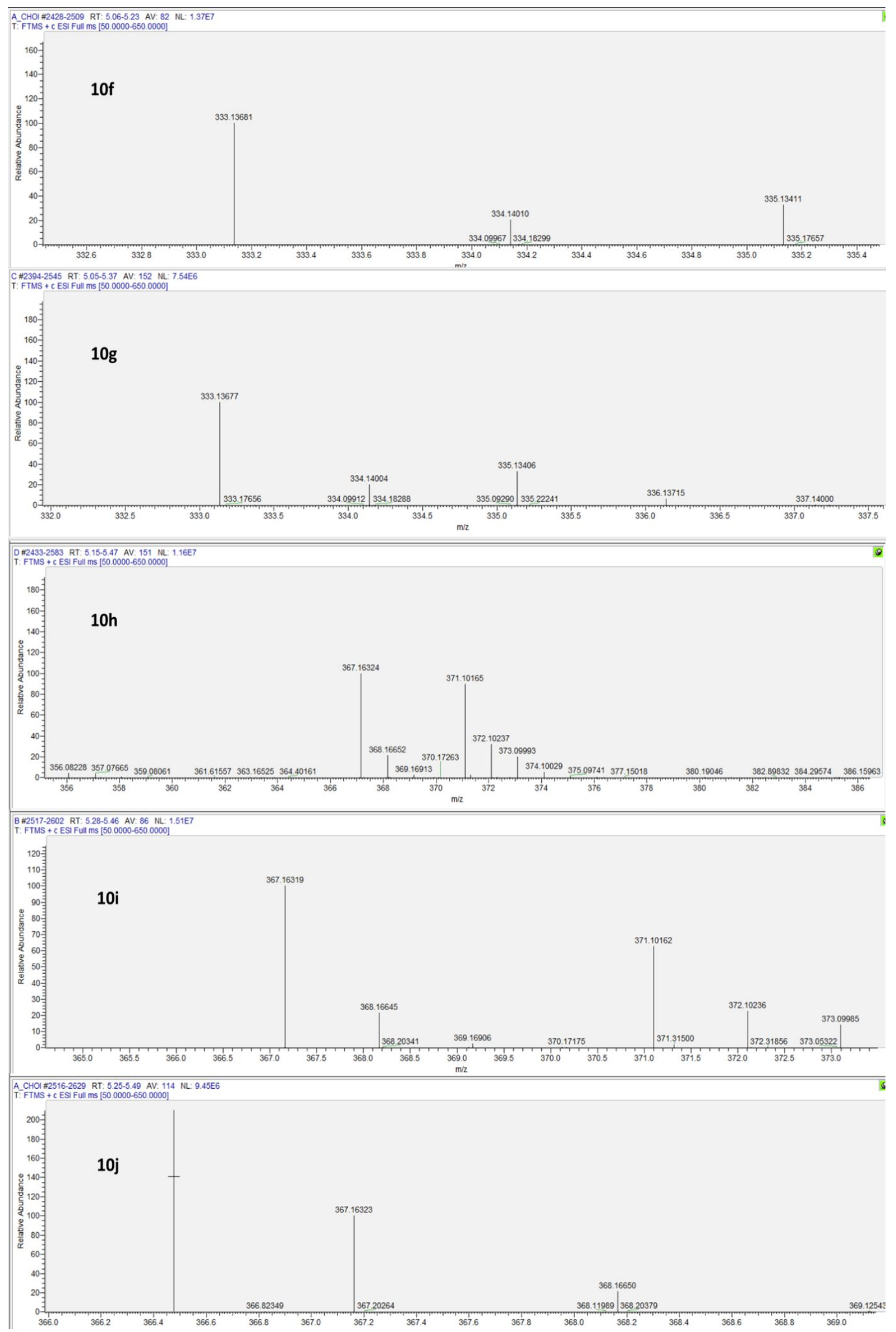




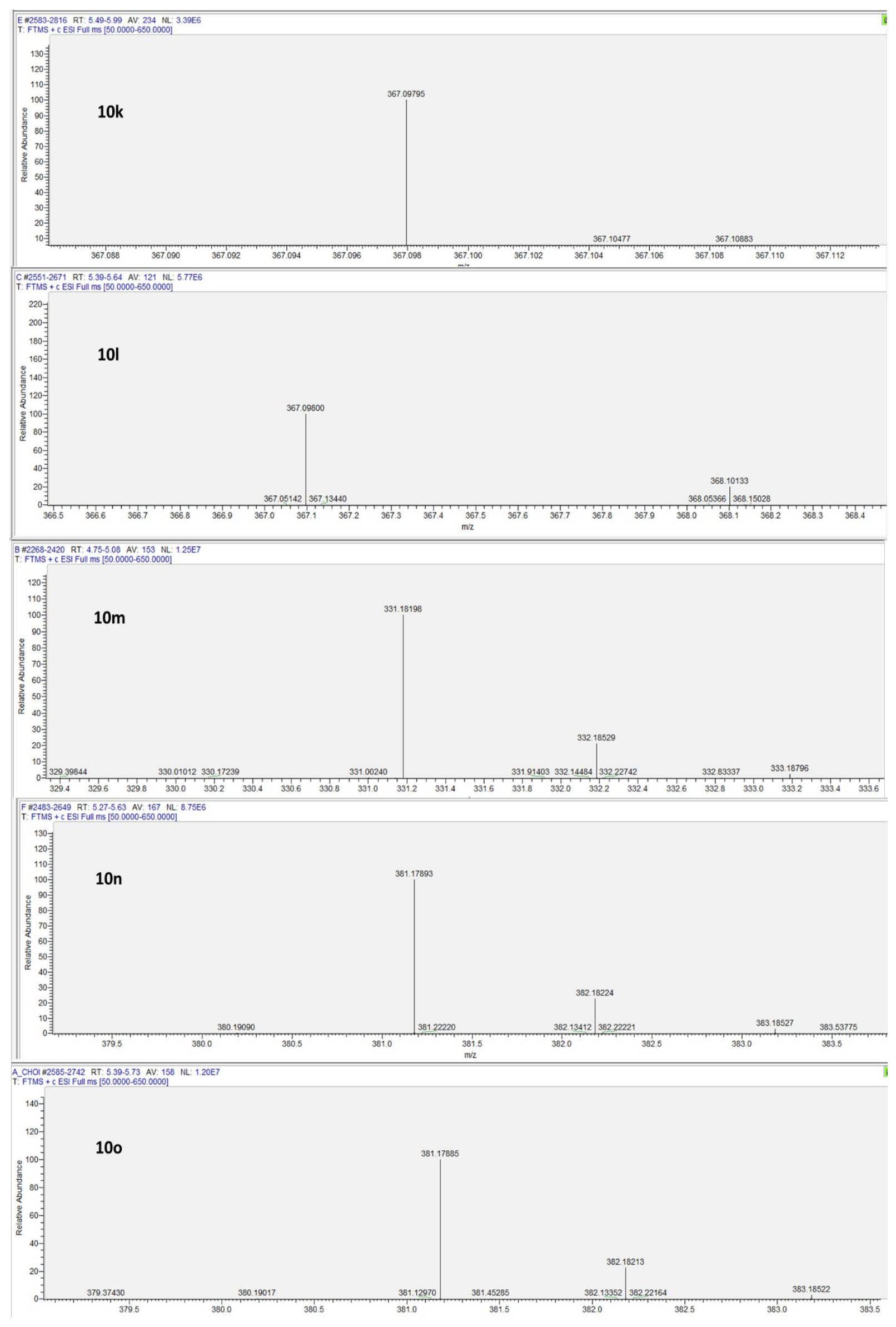



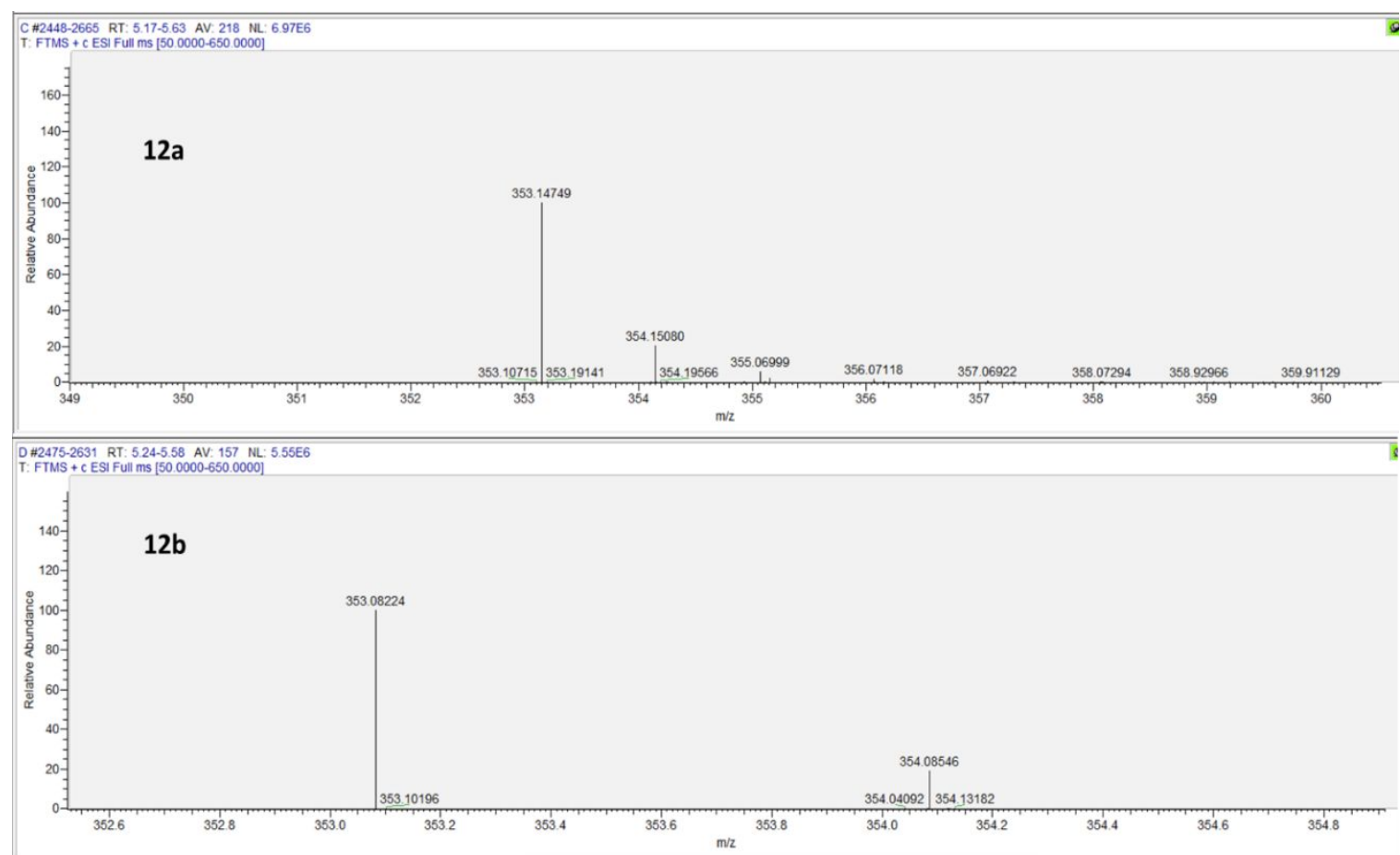

\begin{tabular}{l}
\hline \hline ACHOl \#2441-2591 RT: 520.5 .51 AV: 101 NL: $1.46 E 7$ \\
T. FTMS + C ESI Full mS [50.0000-650.0000]
\end{tabular}
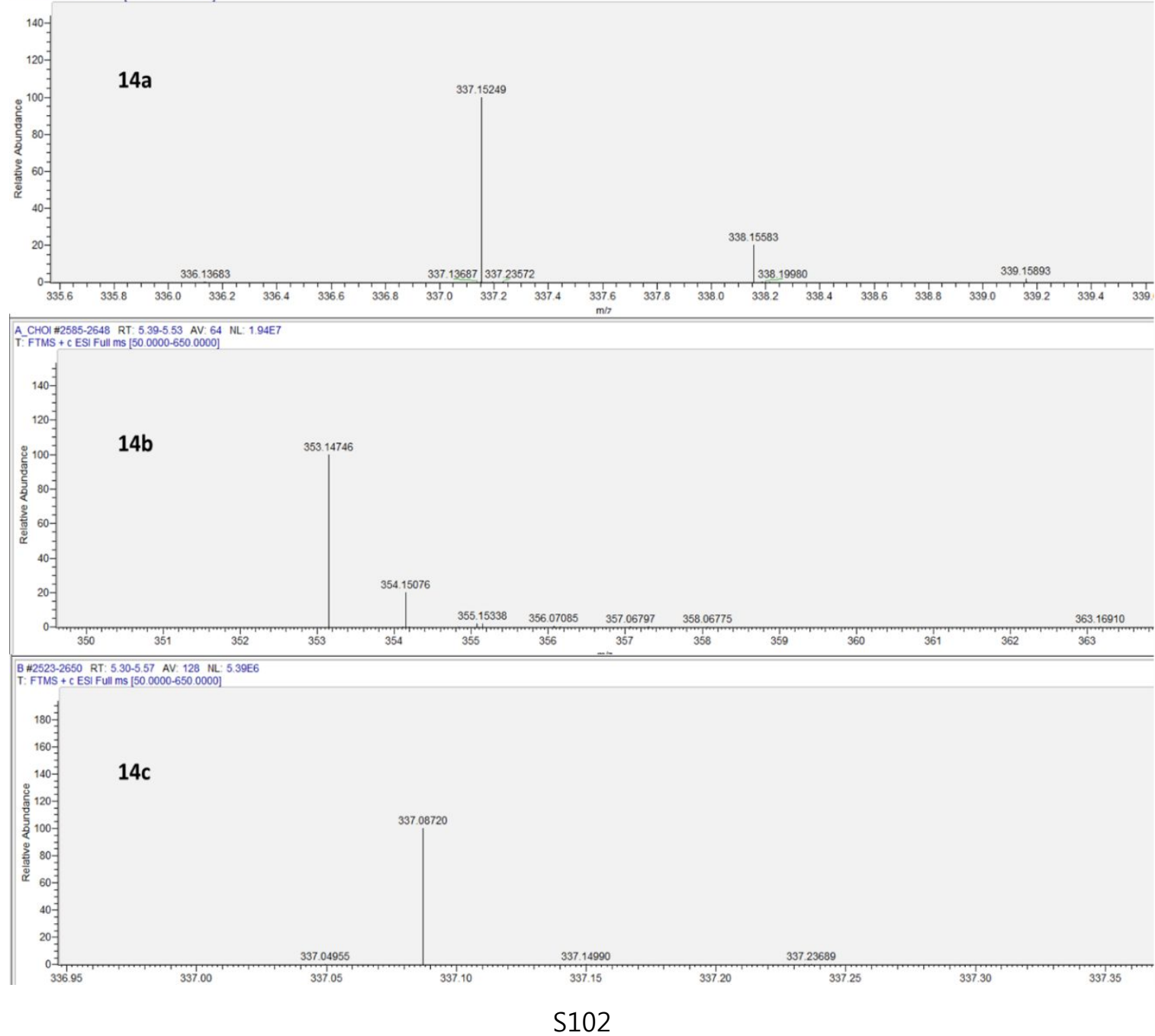

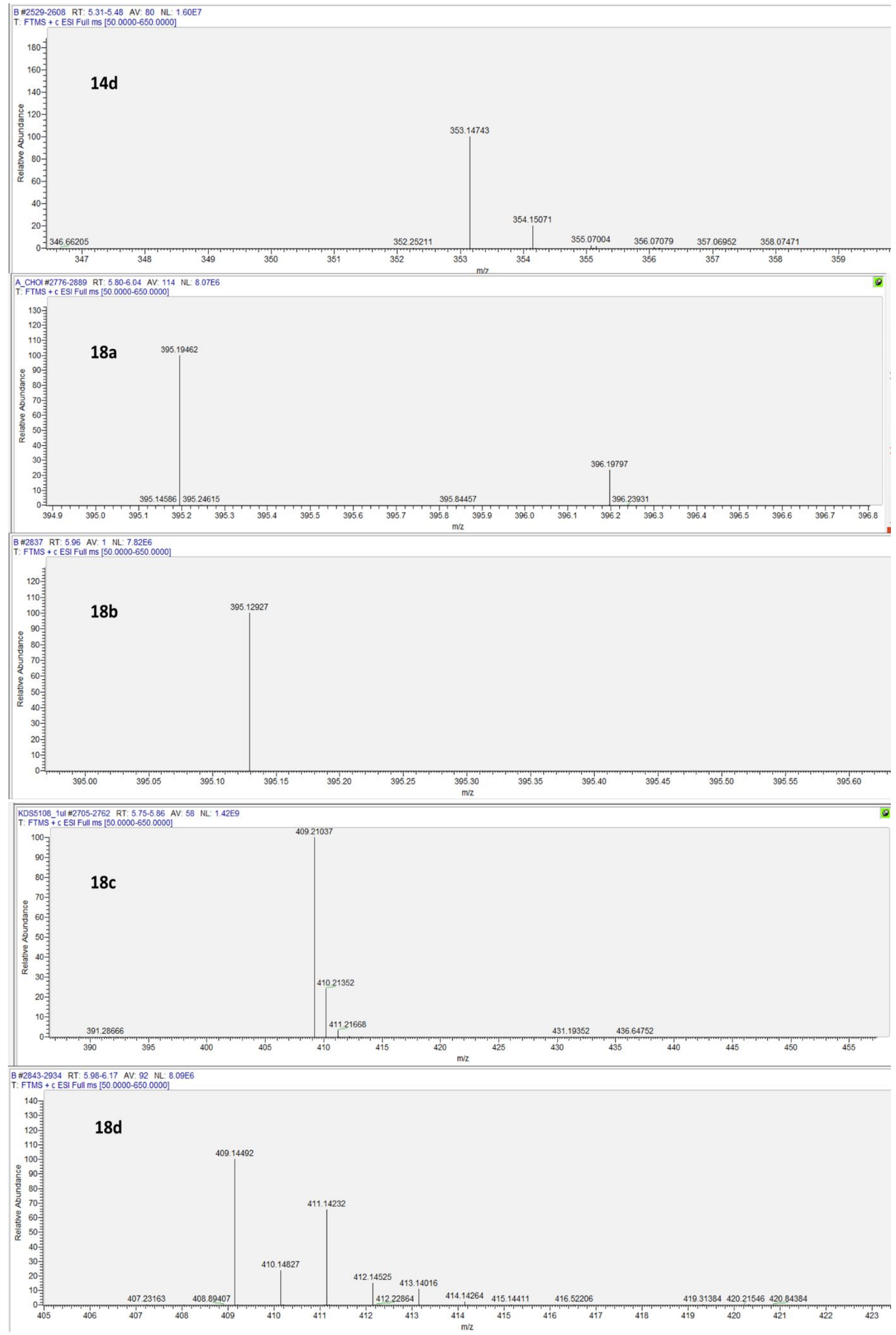


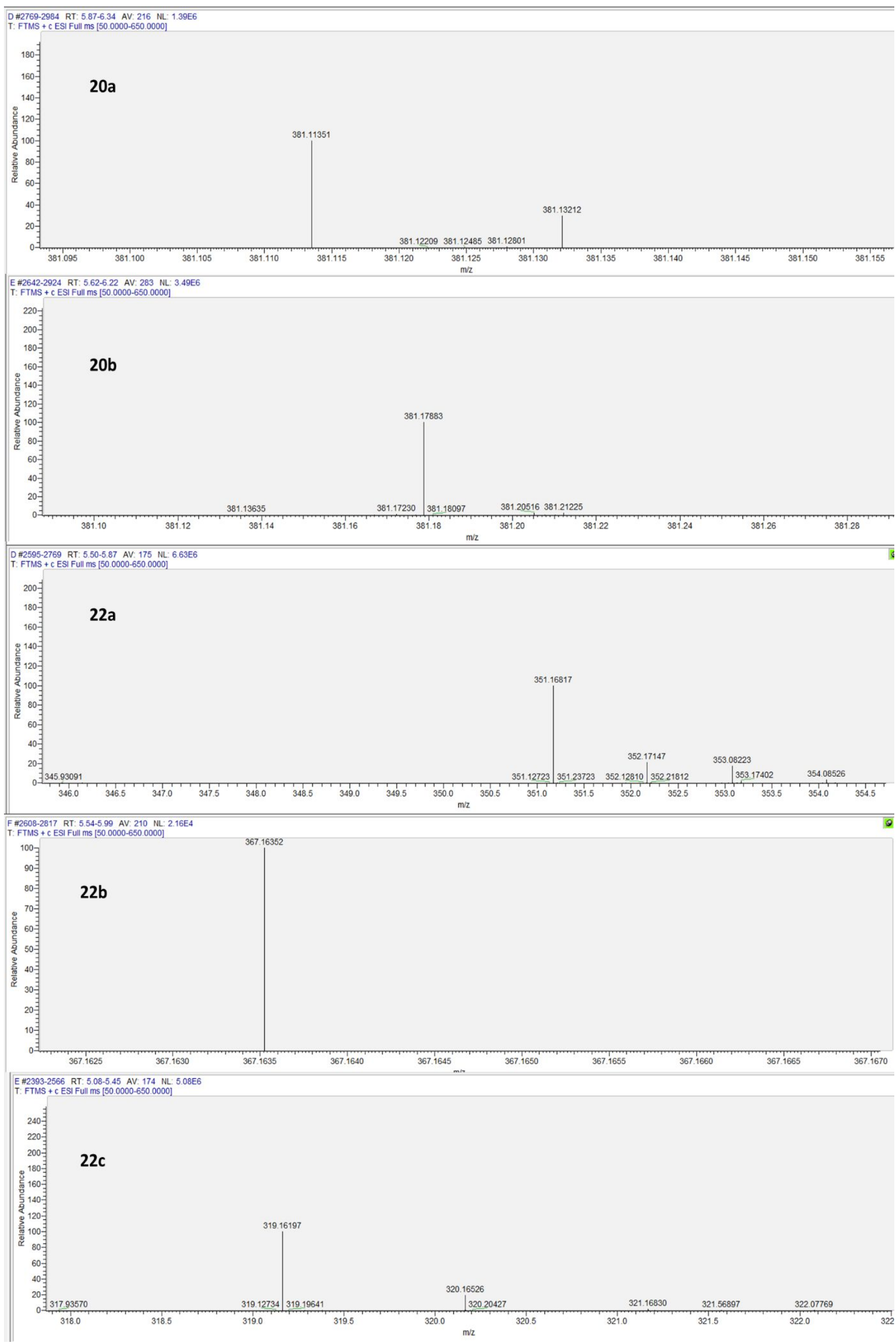




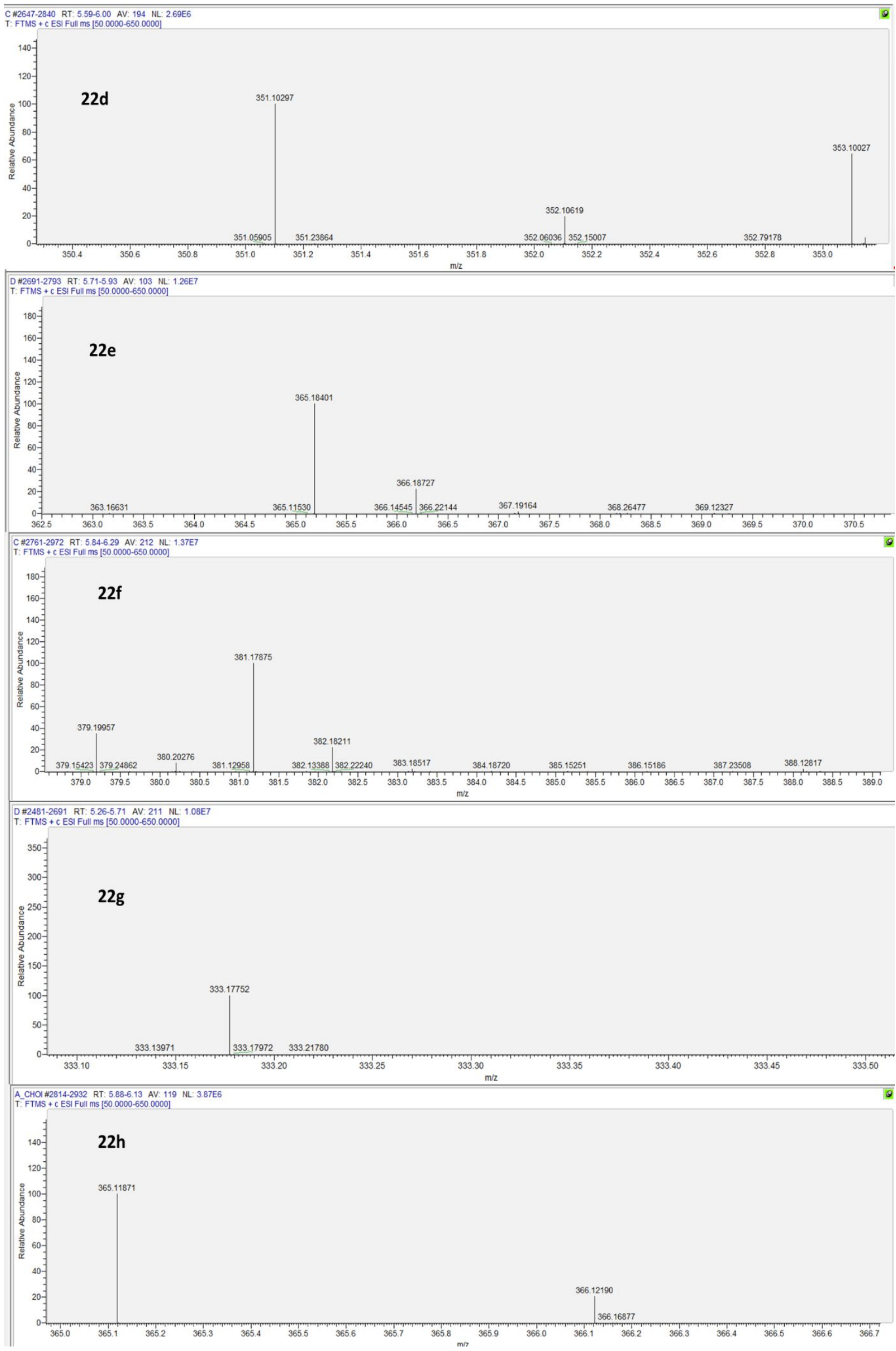




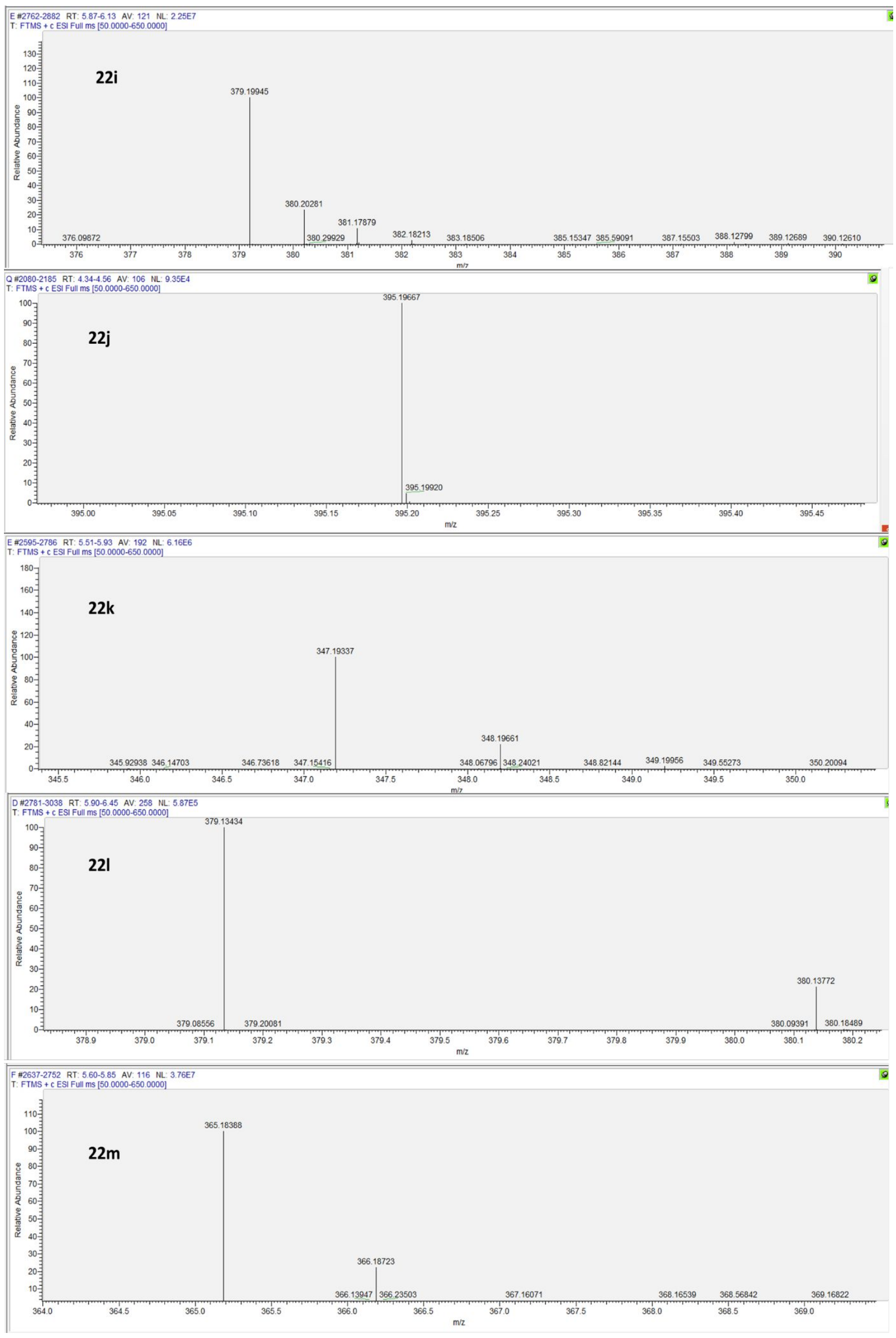




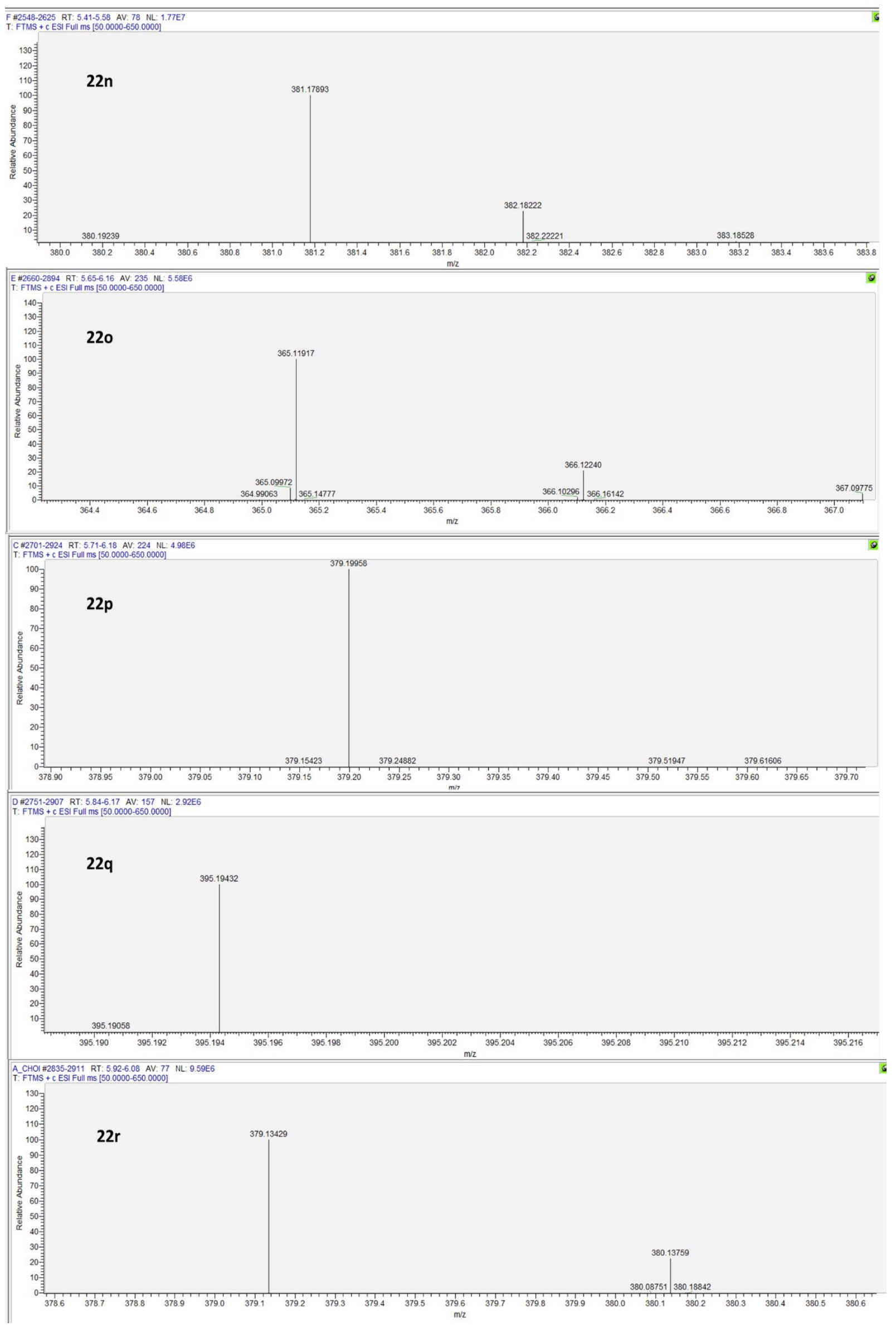




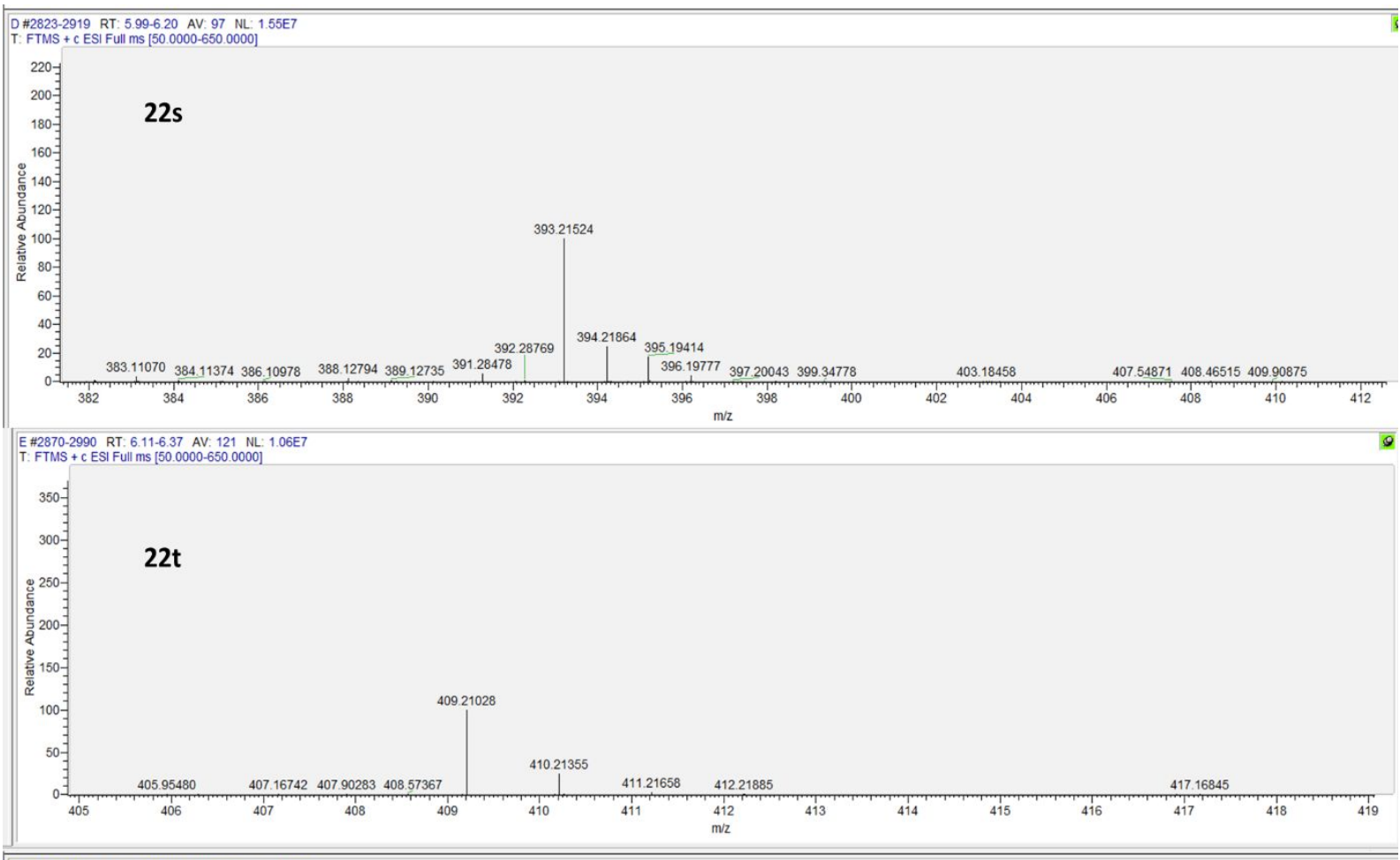

C\#2912-3044 RT: 6 . 16-6.44 AV: 133 NL: 9.98E6

$22 u$

$120=$

然

39314997

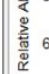

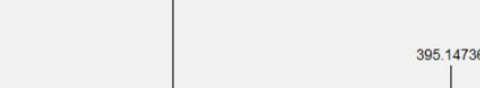

$20=$

3941532

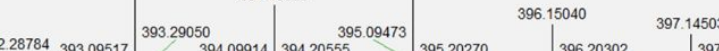

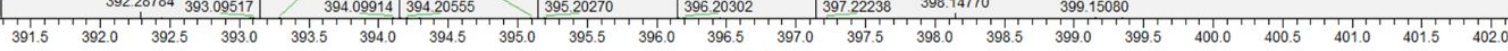

Q \#3599-3696 RT: $7.61-7.82$ AV: 98 NL: 5.10 E6
T: FTTMS + C ESI Full ms [50 00000-650.0000]

$\begin{array}{cc}160 \mathrm{~A} \\ 140= \\ 120= & \mathbf{2 6}\end{array}$

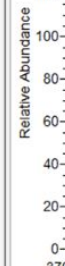

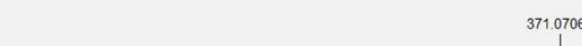

$\stackrel{371.07066}{371.10252}$
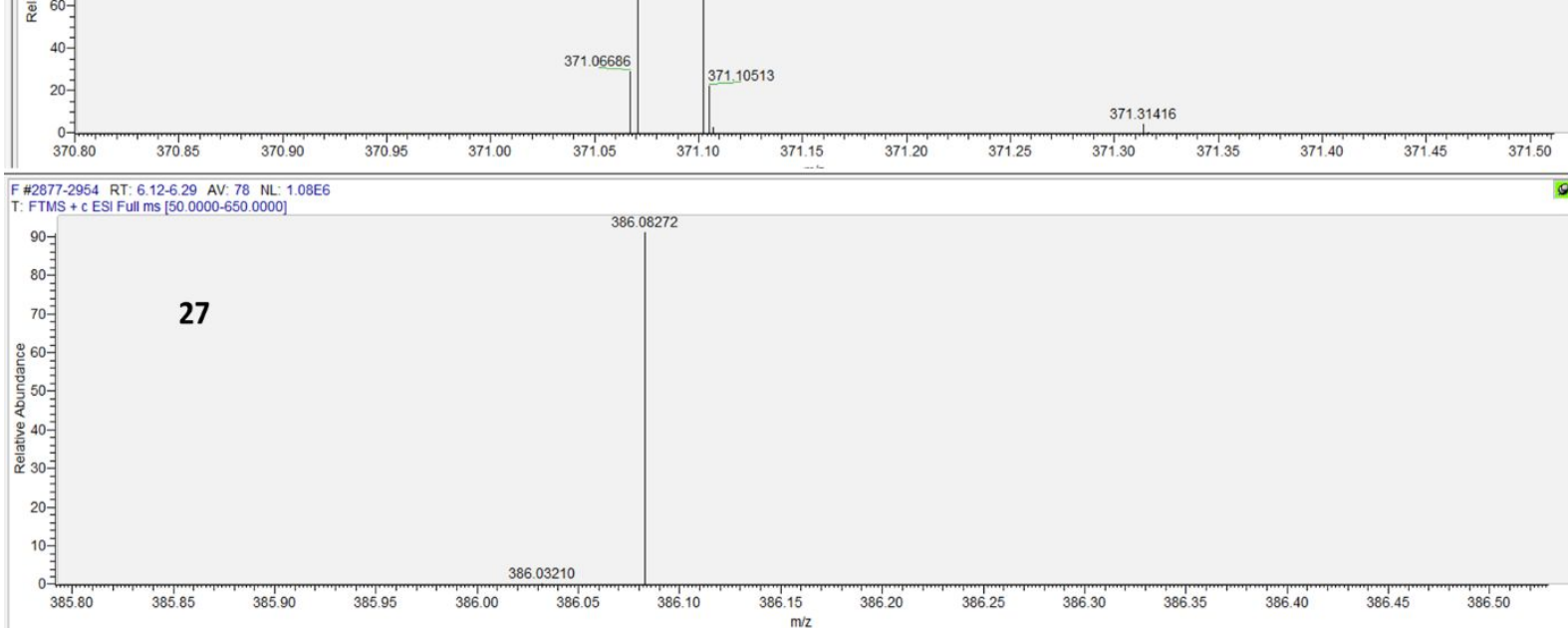


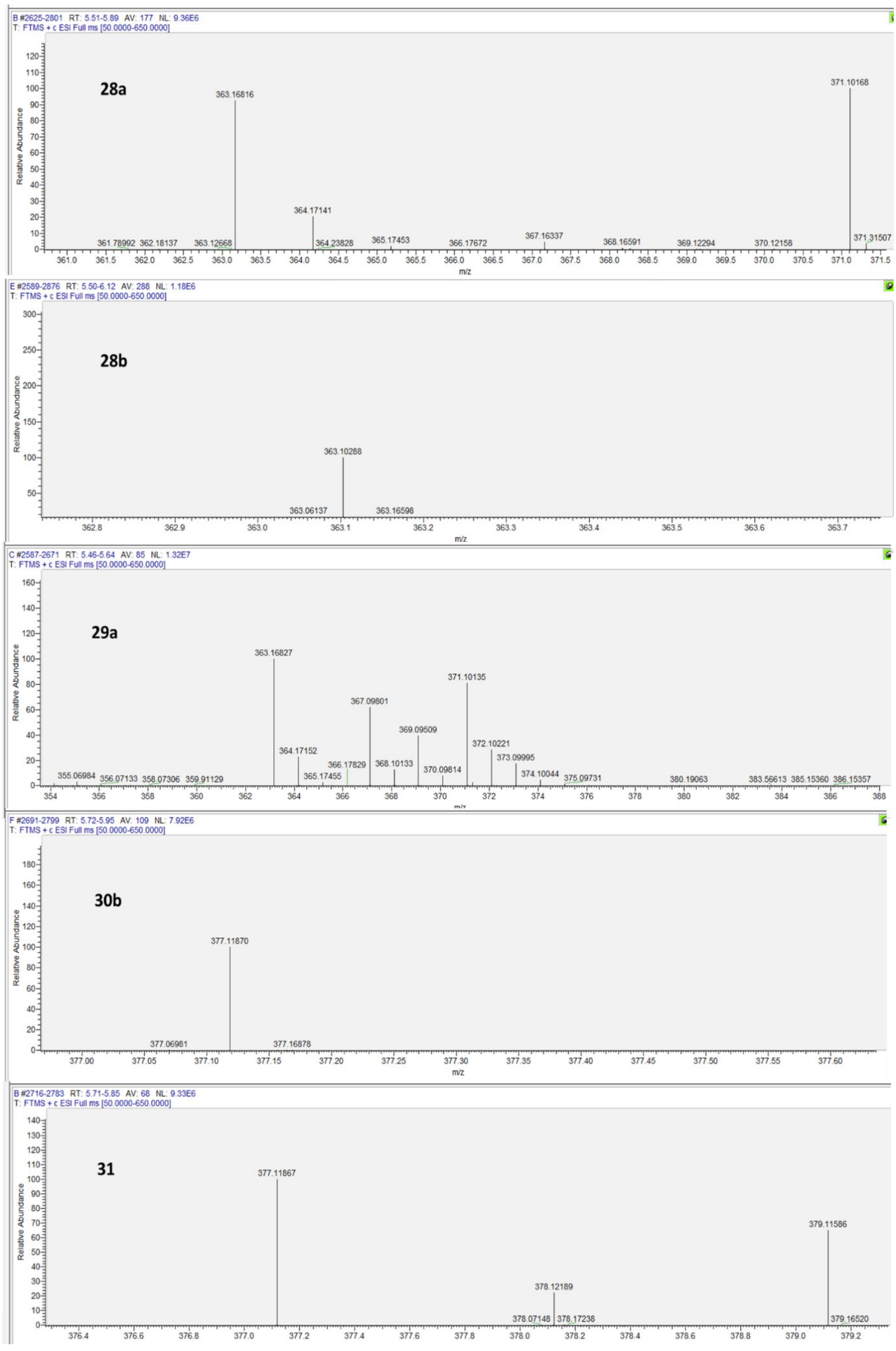




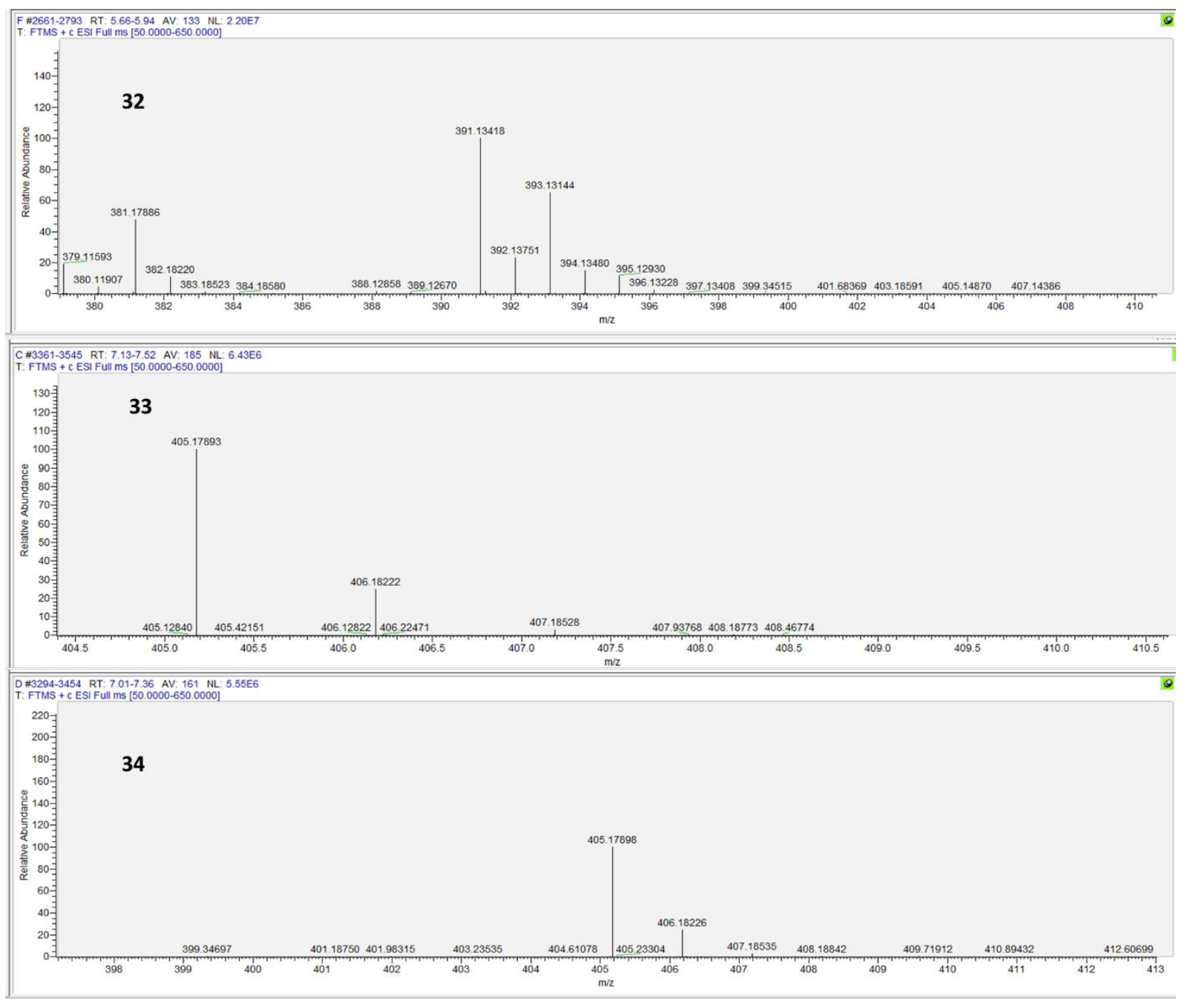




\section{HPLC analysis}
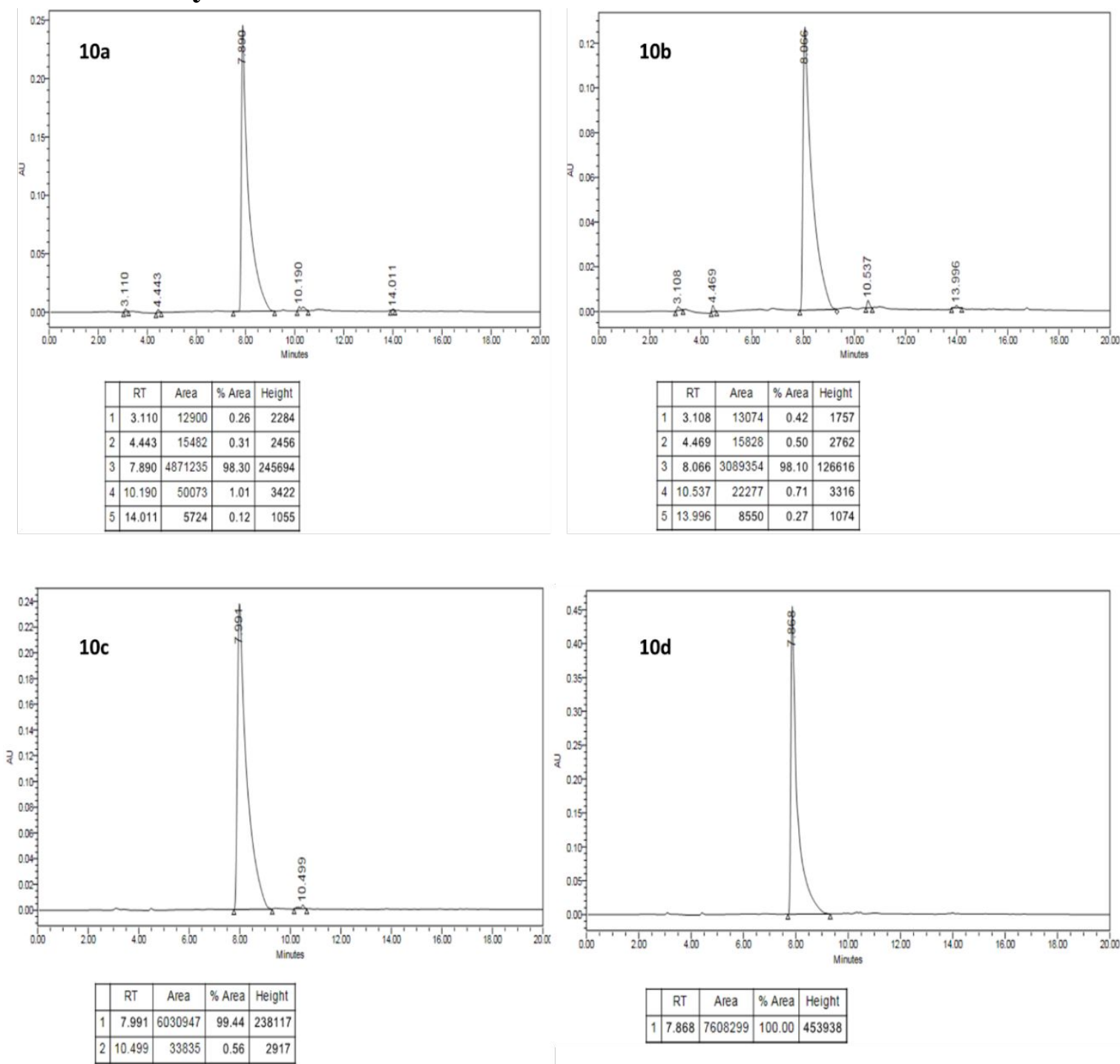

\begin{tabular}{|l|l|l|l|l|}
\hline RT & Area & $\%$ Area & Height \\
\hline
\end{tabular}

\begin{tabular}{|l|l|l|l|l|l|}
\hline 1 & 7.868 & 7608299 & 100.00 & 453938 \\
\hline
\end{tabular}
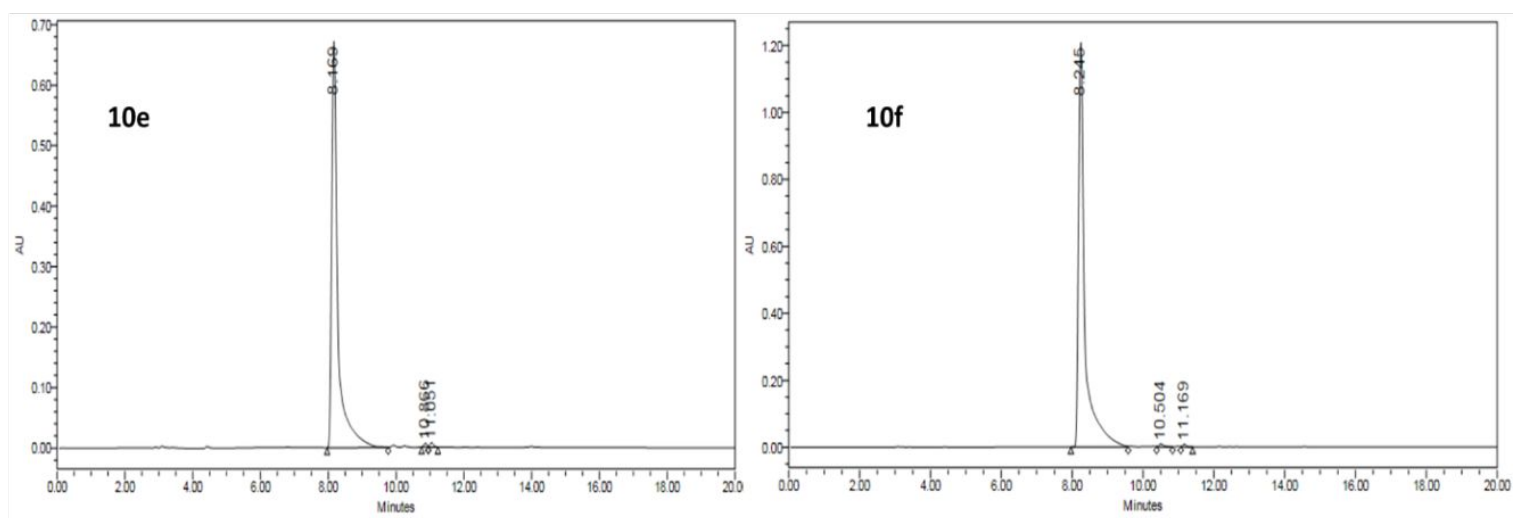

\begin{tabular}{|c|c|r|r|r|}
\hline & \multicolumn{1}{|c|}{ RT } & \multicolumn{1}{c|}{ Area } & \% Area & Height \\
\hline 1 & 8.169 & 8534371 & 98.89 & 671956 \\
\hline 2 & 10.866 & 40840 & 0.47 & 6044 \\
\hline 3 & 11.051 & 54746 & 0.63 & 7310 \\
\hline
\end{tabular}

\begin{tabular}{|l|c|r|r|r|}
\hline & \multicolumn{1}{|c|}{ RT } & \multicolumn{1}{c|}{ Area } & \% Area & Height \\
\hline 1 & 8.245 & 15175674 & 99.04 & 1211454 \\
\hline 2 & 10.504 & 91005 & 0.59 & 9125 \\
\hline 3 & 11.169 & 55926 & 0.36 & 8298 \\
\hline
\end{tabular}



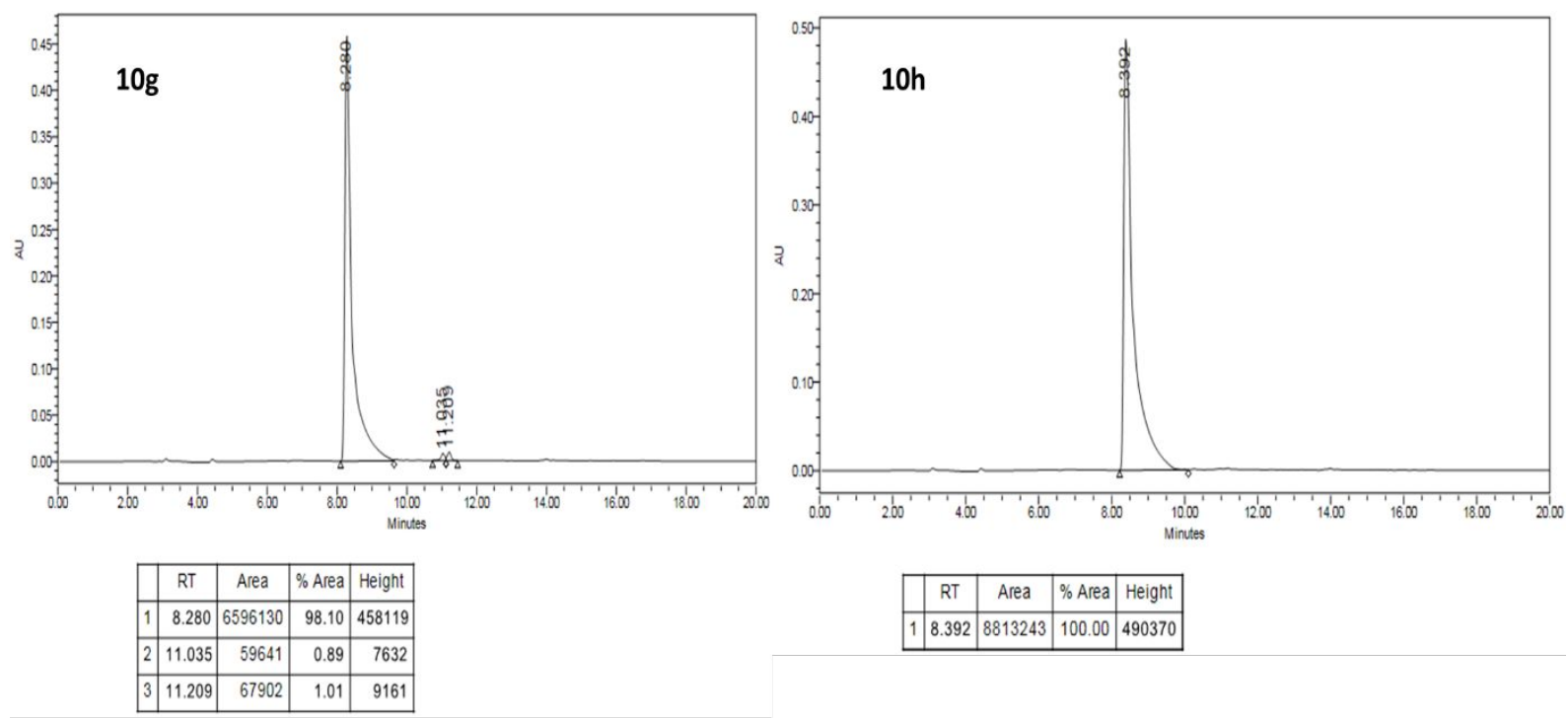

\begin{tabular}{|c|c|c|c|c|}
\hline & RT & Area & $\%$ Area & Height \\
\hline 1 & 8.392 & 8813243 & 10000 & 490370 \\
\hline
\end{tabular}
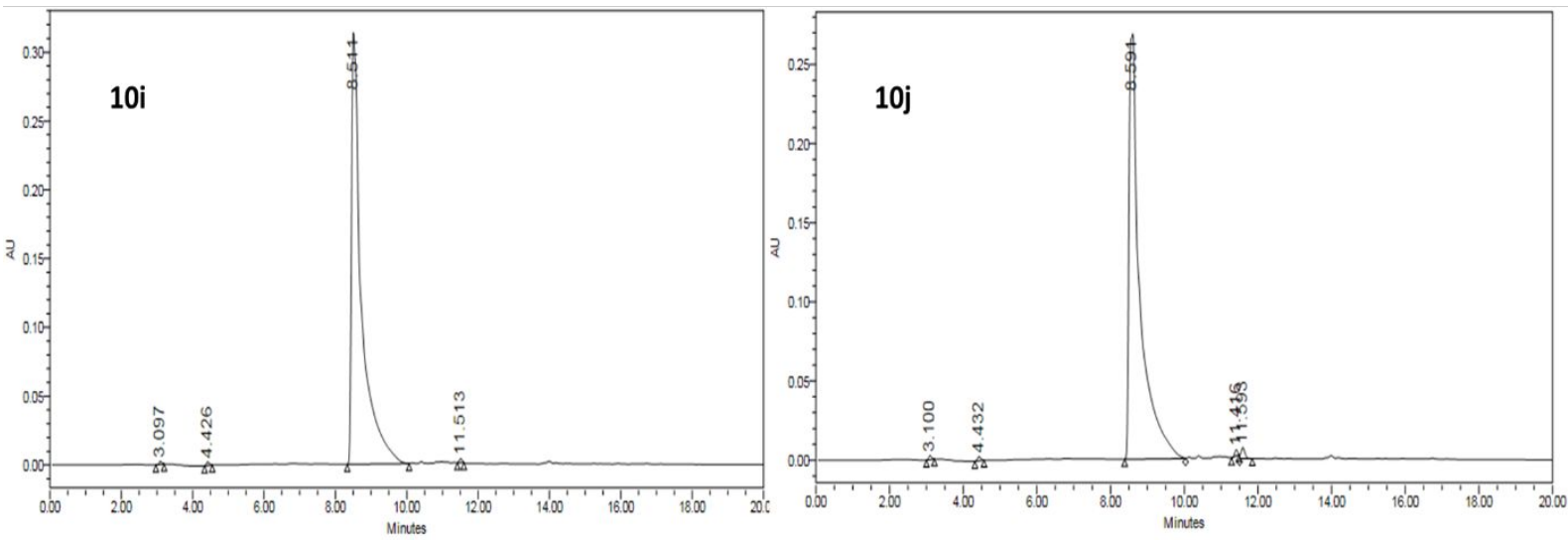

\begin{tabular}{|r|c|r|r|r|}
\hline & RT & Area & \% Area & Height \\
\hline 1 & 3.097 & 12175 & 0.20 & 2158 \\
\hline 2 & 4.426 & 15230 & 0.25 & 2422 \\
\hline 3 & 8.511 & 6119887 & 99.29 & 317860 \\
\hline 4 & 11.513 & 16667 & 0.27 & 2938 \\
\hline
\end{tabular}

\begin{tabular}{|r|c|r|r|r|}
\hline & RT & Area & \% Area & Height \\
\hline 1 & 3.100 & 13808 & 0.24 & 2399 \\
\hline 2 & 4.432 & 16687 & 0.29 & 2532 \\
\hline 3 & 8.591 & 5632154 & 98.25 & 269533 \\
\hline 4 & 11.416 & 19718 & 0.34 & 3735 \\
\hline 5 & 11.593 & 50012 & 0.87 & 6856 \\
\hline
\end{tabular}
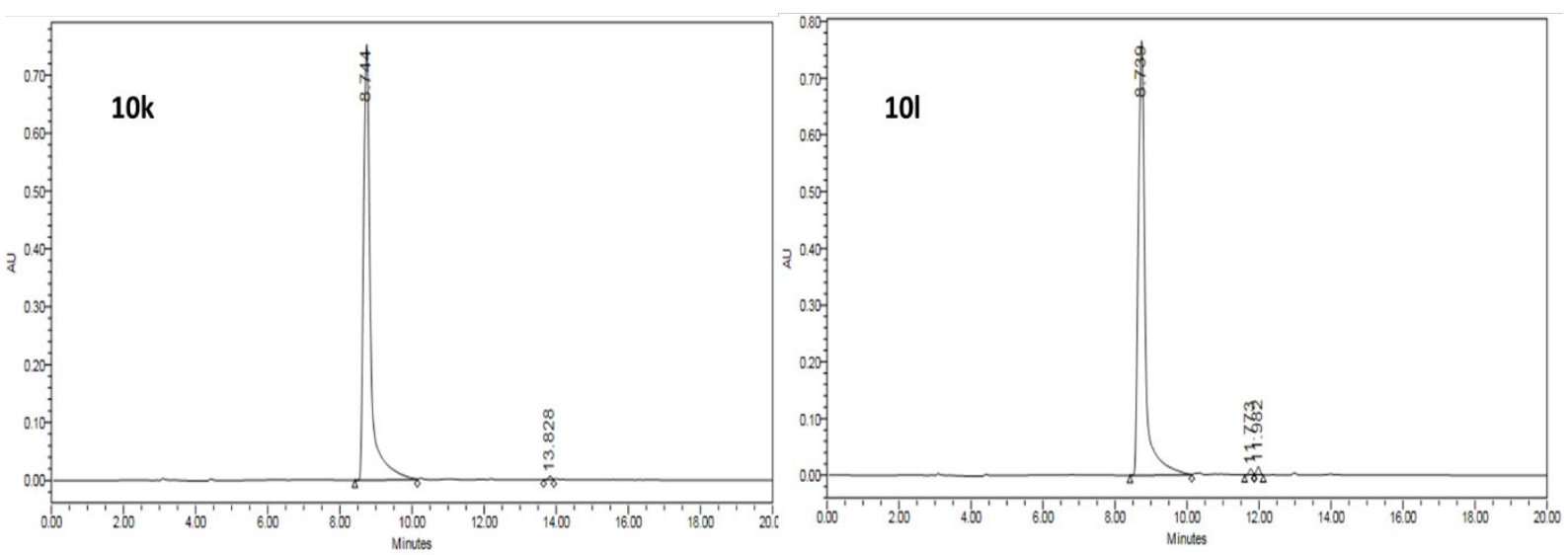

\begin{tabular}{|c|c|r|r|r|}
\hline & \multicolumn{1}{|c|}{ RT } & \multicolumn{1}{c|}{ Area } & \% Area & Height \\
\hline 1 & 8.744 & 10274387 & 99.55 & 754380 \\
\hline 2 & 13.828 & 46266 & 0.45 & 6441 \\
\hline
\end{tabular}

\begin{tabular}{|l|c|r|r|r|}
\hline & \multicolumn{1}{|c|}{ RT } & \multicolumn{1}{|c|}{ Area } & \% Area & Height \\
\hline 1 & 8.739 & 10352435 & 98.41 & 767325 \\
\hline 2 & 11.773 & 71240 & 0.68 & 10631 \\
\hline 3 & 11.982 & 95886 & 0.91 & 13892 \\
\hline
\end{tabular}



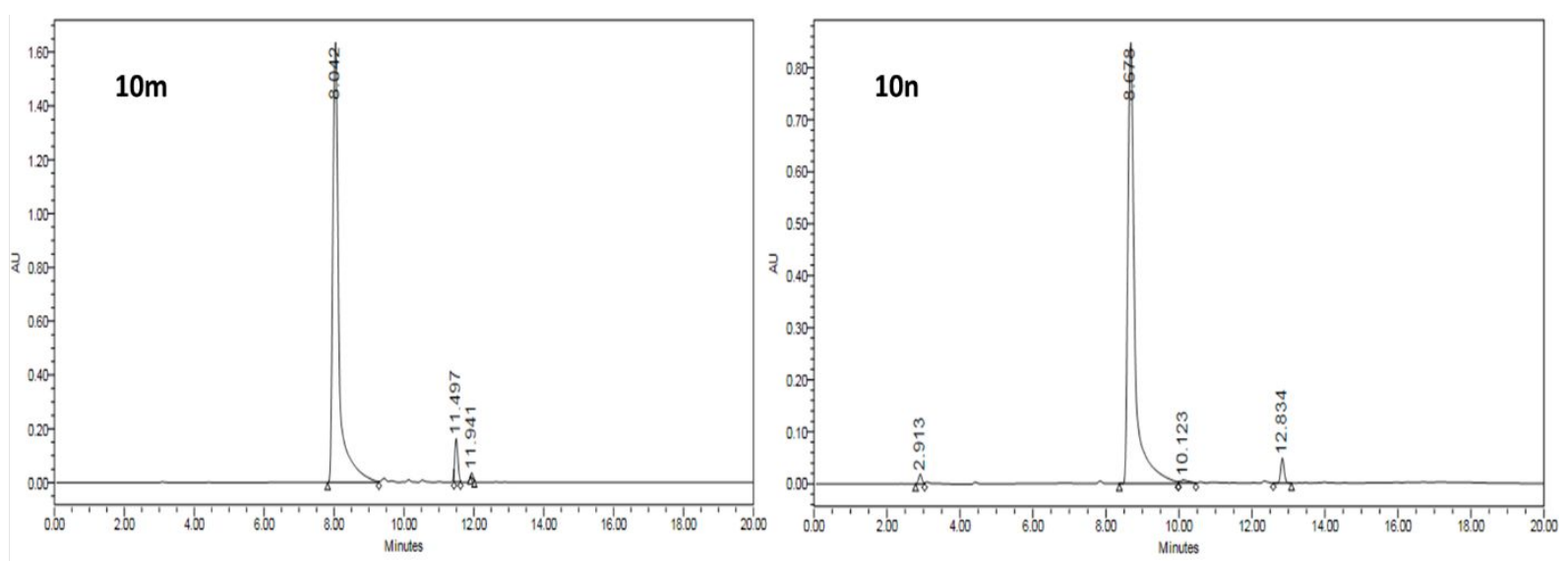

\begin{tabular}{|l|c|r|r|r|}
\hline & RT & \multicolumn{1}{|c|}{ Area } & \% Area & Height \\
\hline 1 & 8.042 & 20345298 & 95.14 & 1646330 \\
\hline 2 & 11.497 & 965861 & 4.52 & 160652 \\
\hline 3 & 11.941 & 72545 & 0.34 & 17340 \\
\hline
\end{tabular}

$$
\begin{array}{|r|c|r|r|r|}
\hline & \text { RT } & \multicolumn{1}{|c|}{\text { Area }} & \text { \% Area } & \text { Height } \\
\hline 1 & 2.913 & 112435 & 0.94 & 18081 \\
\hline 2 & 8.678 & 11390509 & 95.46 & 849314 \\
\hline 3 & 10.123 & 108406 & 0.91 & 6421 \\
\hline 4 & 12.834 & 321014 & 2.69 & 47865 \\
\hline
\end{array}
$$
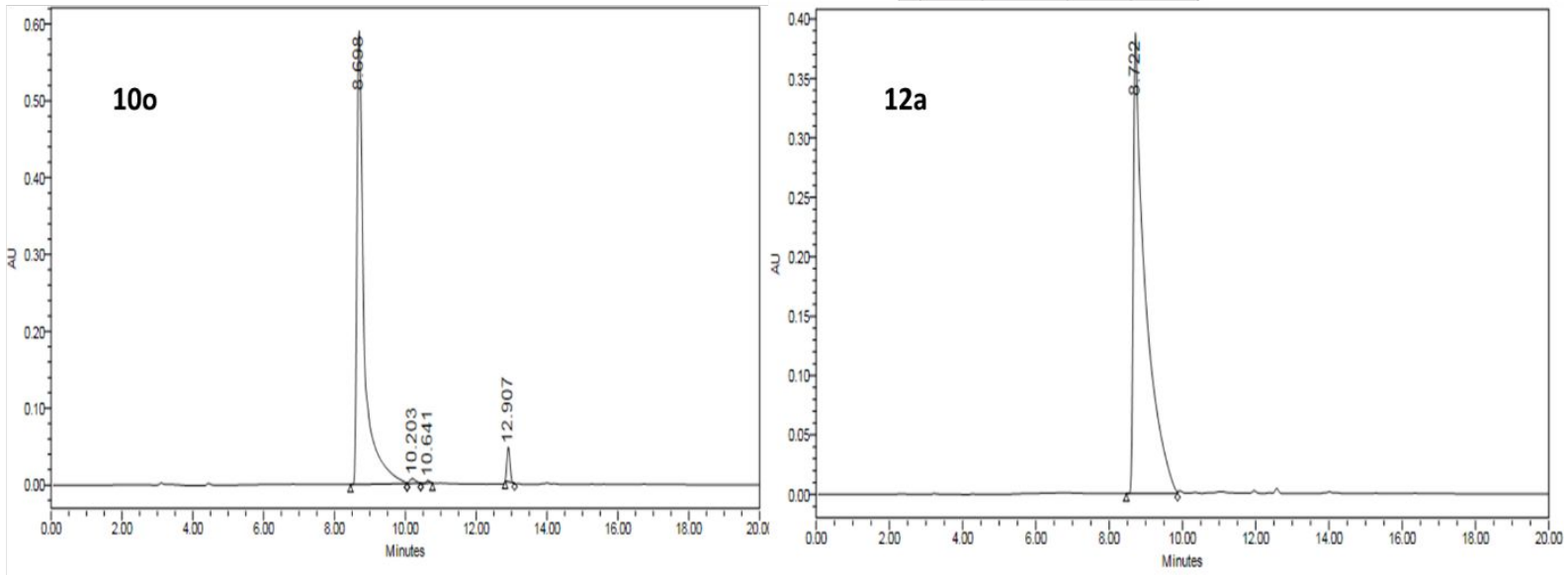

\begin{tabular}{|r|r|r|r|r|}
\hline & \multicolumn{1}{|c|}{ RT } & \multicolumn{1}{|c|}{ Area } & \% Area & Height \\
\hline 1 & 8.698 & 8484936 & 95.74 & 590453 \\
\hline 2 & 10.203 & 73509 & 0.83 & 6062 \\
\hline 3 & 10.641 & 27949 & 0.32 & 3501 \\
\hline 4 & 12.907 & 276214 & 3.12 & 44854 \\
\hline
\end{tabular}
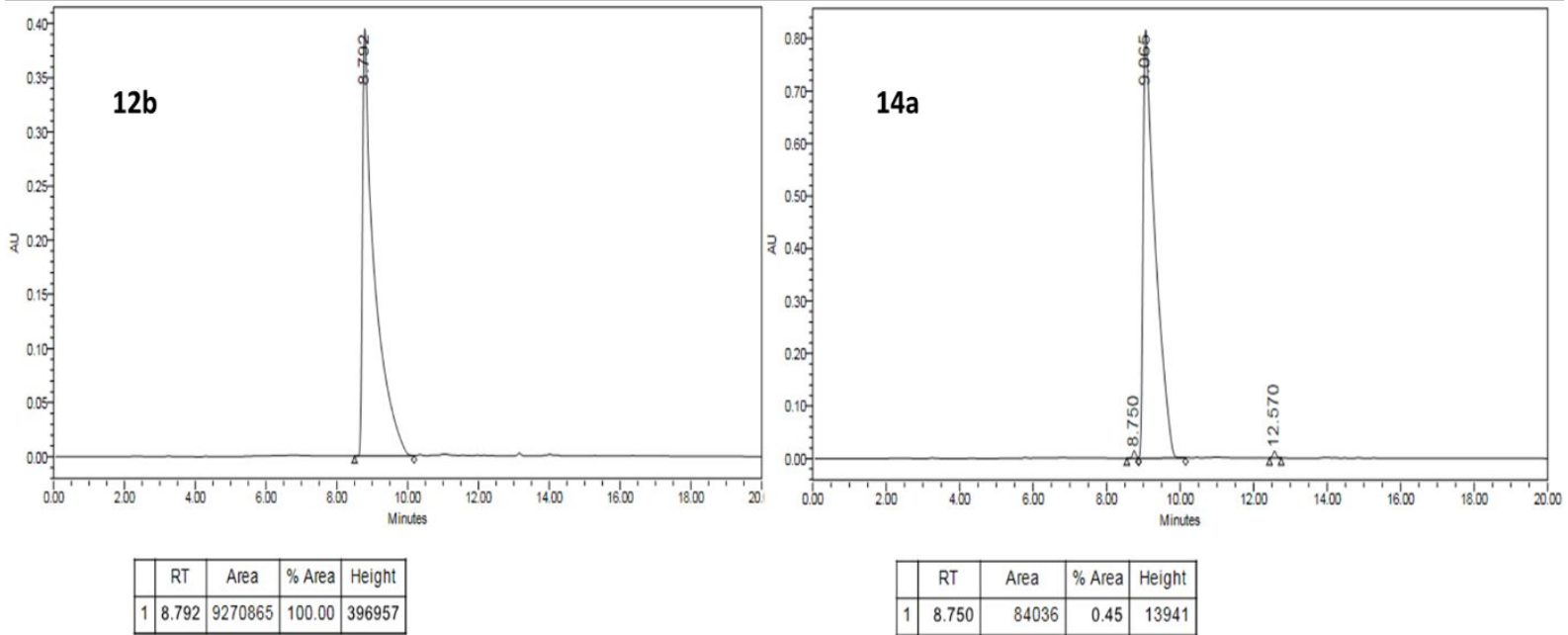

\begin{tabular}{|r|c|r|r|r|}
\hline & RT & \multicolumn{1}{|c|}{ Area } & \% Area & Height \\
\hline 1 & 8.750 & 84036 & 0.45 & 13941 \\
\hline 2 & 9.065 & 18556165 & 99.10 & 815767 \\
\hline 3 & 12.570 & 83946 & 0.45 & 12862 \\
\hline
\end{tabular}



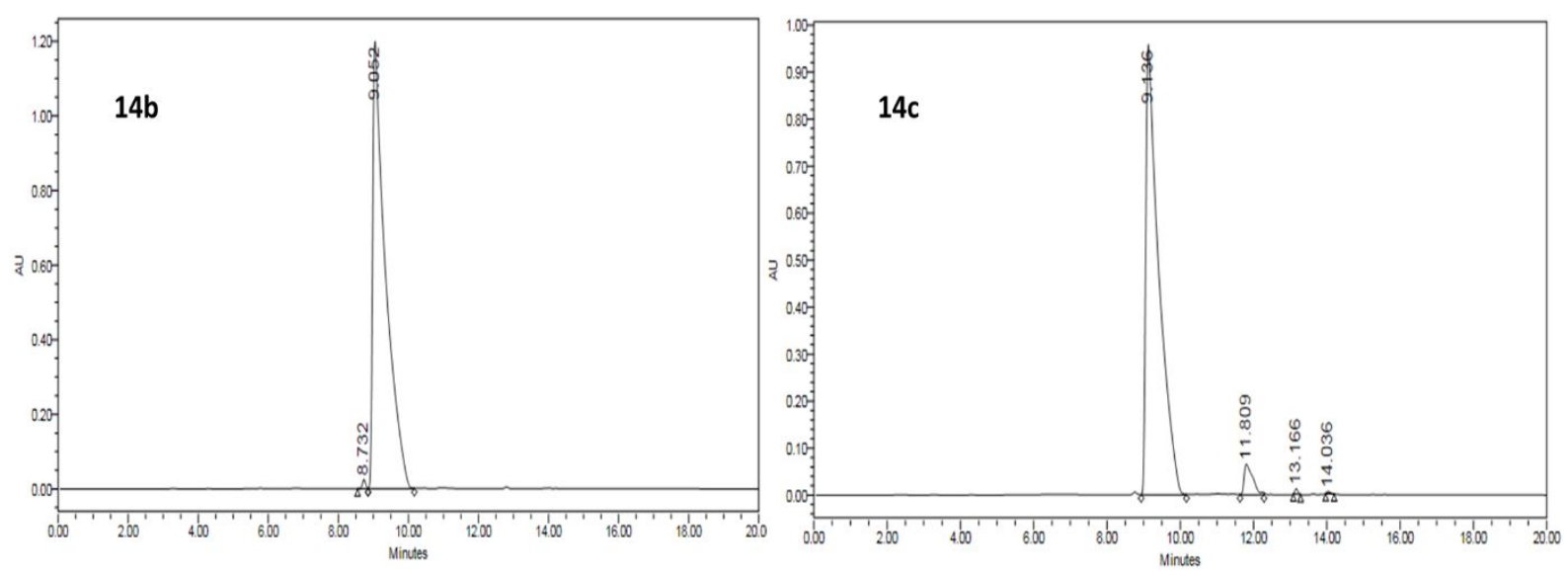

\begin{tabular}{|c|c|r|r|r|}
\hline RT & \multicolumn{1}{|c|}{ Area } & \% Area & Height \\
\hline 1 & 8.732 & 151974 & 0.50 & 24748 \\
\hline 2 & 9.052 & 30325102 & 99.50 & 1199686 \\
\hline
\end{tabular}

\begin{tabular}{|l|c|r|r|r|}
\hline & \multicolumn{1}{|c|}{ RT } & \multicolumn{1}{c|}{ Area } & \% Area & Height \\
\hline 1 & 9.136 & 23634525 & 95.39 & 958266 \\
\hline 2 & 11.809 & 1046934 & 4.23 & 64918 \\
\hline 3 & 13.166 & 66300 & 0.27 & 1110 \\
\hline 4 & 14.036 & 28599 & 0.12 & 4332 \\
\hline
\end{tabular}
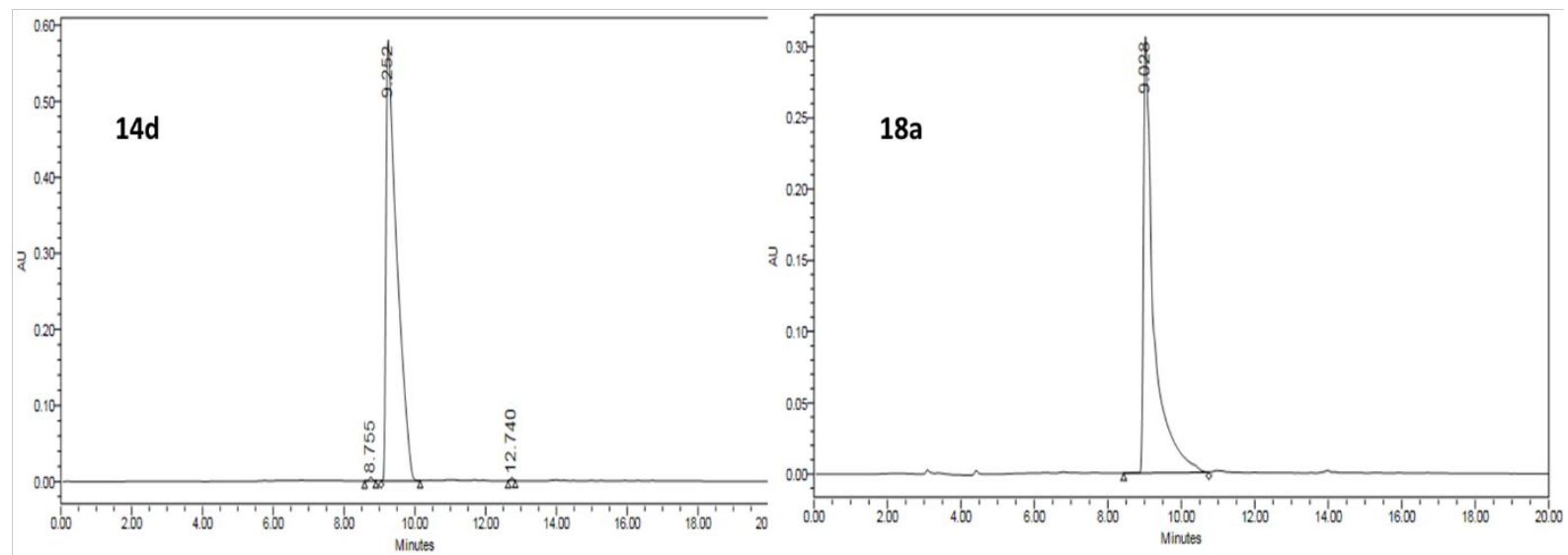

\begin{tabular}{|l|c|r|r|r|}
\hline & RT & \multicolumn{1}{|c|}{ Area } & \% Area & Height \\
\hline 1 & 8.755 & 27163 & 0.22 & 4606 \\
\hline 2 & 9.252 & 12232901 & 99.60 & 580055 \\
\hline 3 & 12.740 & 22499 & 0.18 & 3770 \\
\hline
\end{tabular}

\begin{tabular}{l|l|l|l|l|}
\hline RT & Area & $\%$ Area & Height \\
\hline
\end{tabular}

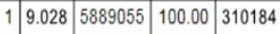
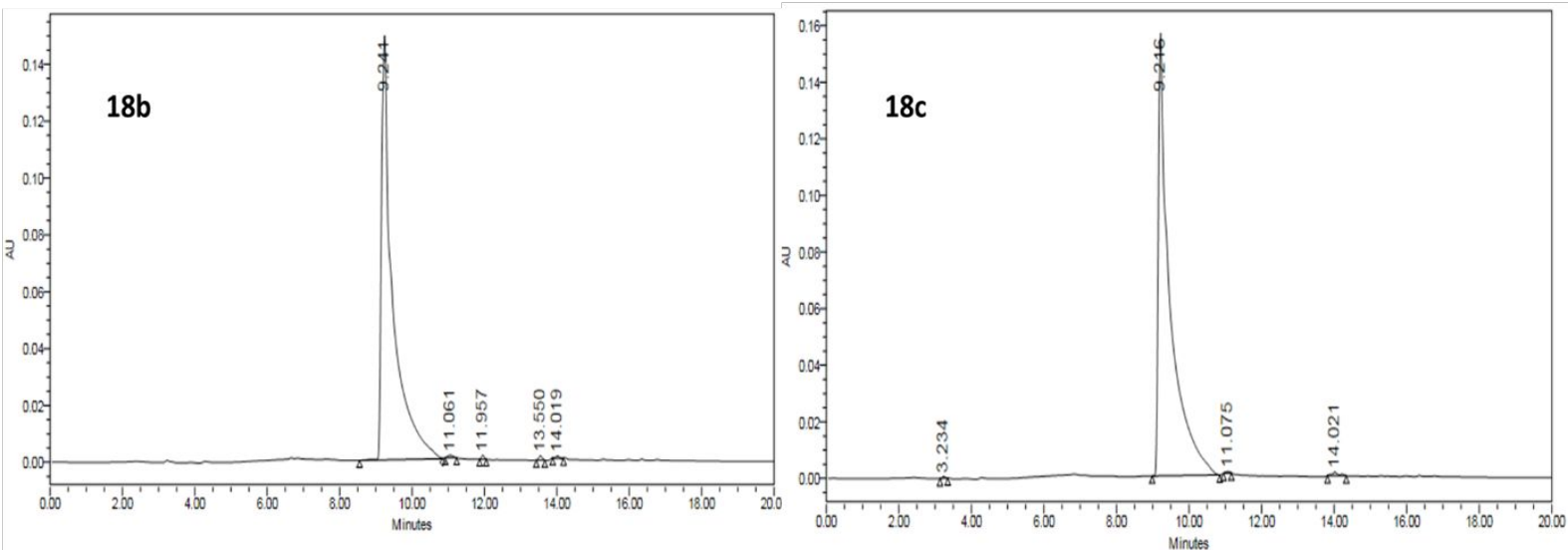

\begin{tabular}{|l|l|r|r|r|}
\hline & RT & \multicolumn{1}{|c|}{ Area } & \% Area & Height \\
\hline 1 & 9.241 & 3216929 & 99.02 & 149998 \\
\hline 2 & 11.061 & 9464 & 0.29 & 819 \\
\hline 3 & 11.957 & 7601 & 0.23 & 1301 \\
\hline 4 & 13.550 & 9772 & 0.30 & 1541 \\
\hline 5 & 14.019 & 5047 & 0.16 & 798 \\
\hline
\end{tabular}

\begin{tabular}{|l|c|r|r|r|}
\hline & RT & Area & \% Area & Height \\
\hline 1 & 3.234 & 3612 & 0.10 & 587 \\
\hline 2 & 9.216 & 3484173 & 99.30 & 156172 \\
\hline 3 & 11.075 & 4317 & 0.12 & 503 \\
\hline 4 & 14.021 & 16456 & 0.47 & 1240 \\
\hline
\end{tabular}



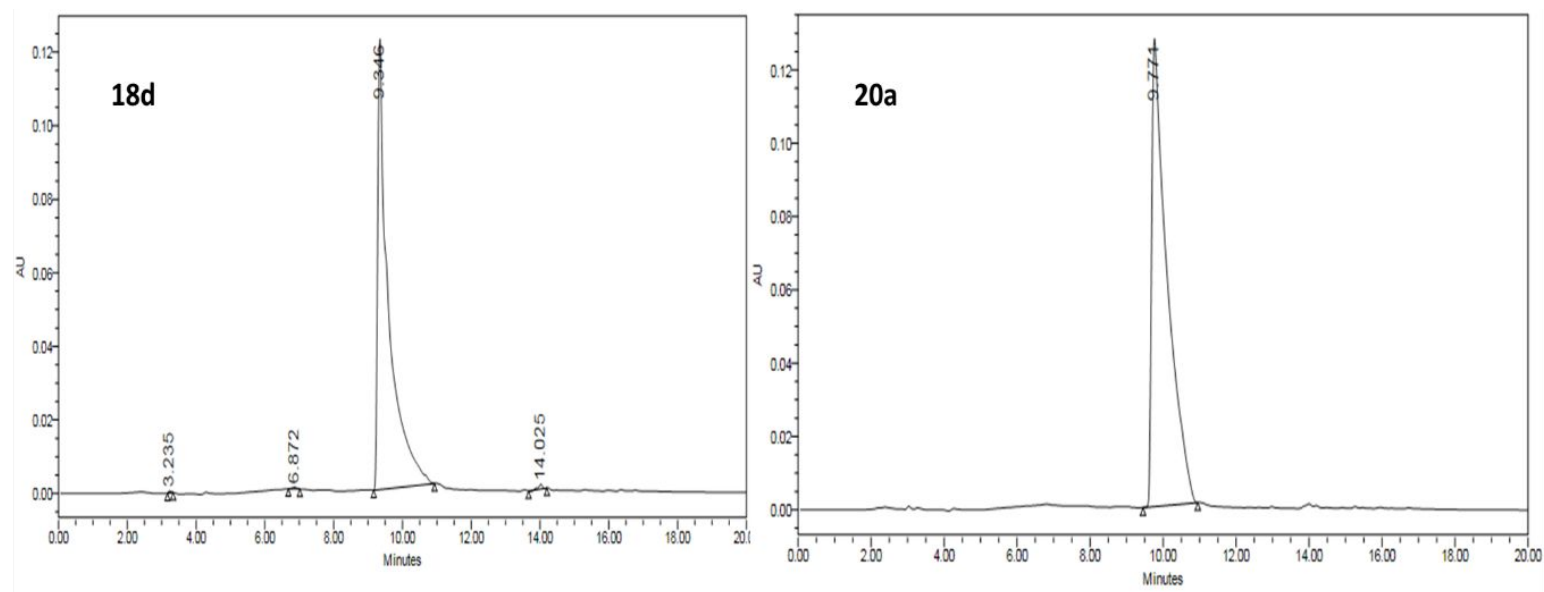

\begin{tabular}{|l|l|r|r|r|}
\hline & RT & Area & $\%$ Area & Height \\
\hline 1 & 3.235 & 2786 & 0.10 & 522 \\
\hline 2 & 6.872 & 4396 & 0.16 & 392 \\
\hline 3 & 9.346 & 2726416 & 99.29 & 122509 \\
\hline 4 & 14.025 & 12317 & 0.45 & 1238 \\
\hline
\end{tabular}
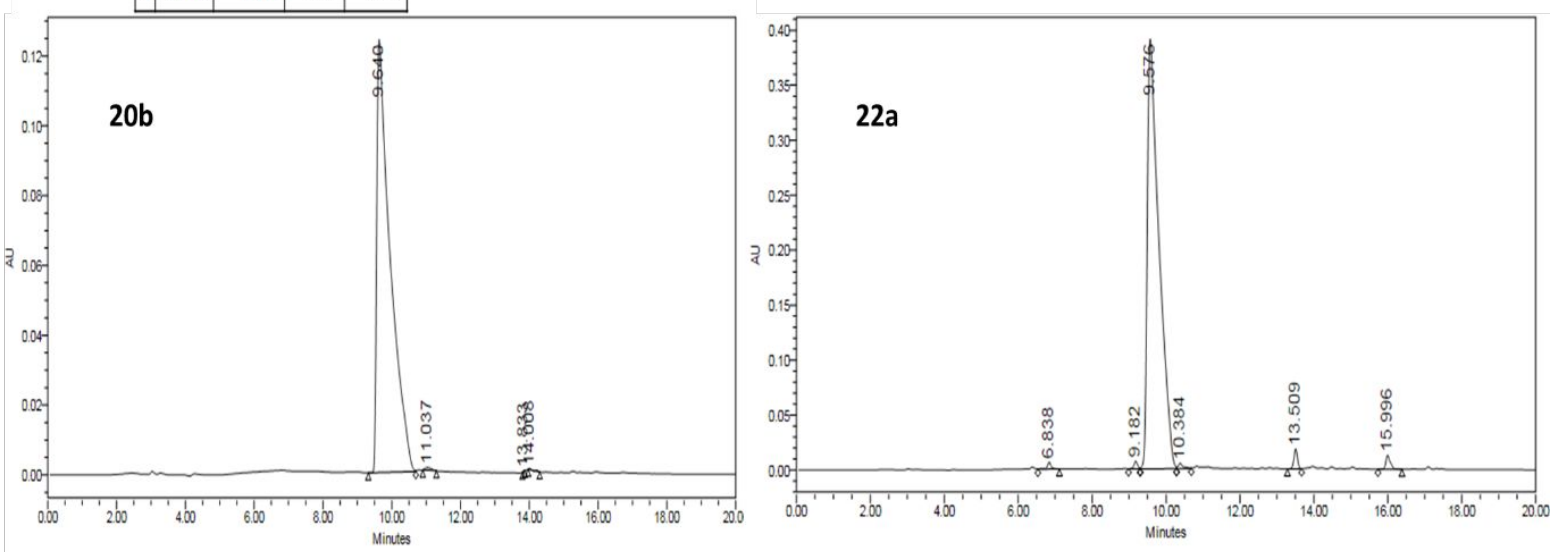

\begin{tabular}{|l|c|r|r|r|}
\hline & RT & \multicolumn{1}{c|}{ Area } & \% Area & Height \\
\hline 1 & 9.640 & 3363756 & 99.61 & 124371 \\
\hline 2 & 11.037 & 9863 & 0.29 & 712 \\
\hline 3 & 13.833 & 160 & 0.00 & 66 \\
\hline 4 & 14.008 & 3161 & 0.09 & 304 \\
\hline
\end{tabular}

\begin{tabular}{|r|c|r|r|r|}
\hline & RT & Area & $\%$ Area & Height \\
\hline 1 & 6.838 & 44964 & 0.50 & 6145 \\
\hline 2 & 9.182 & 46231 & 0.52 & 6937 \\
\hline 3 & 9.576 & 8567001 & 95.93 & 392685 \\
\hline 4 & 10.384 & 43145 & 0.48 & 4628 \\
\hline 5 & 13.509 & 122969 & 1.38 & 18230 \\
\hline 6 & 15.996 & 106366 & 1.19 & 12532 \\
\hline
\end{tabular}
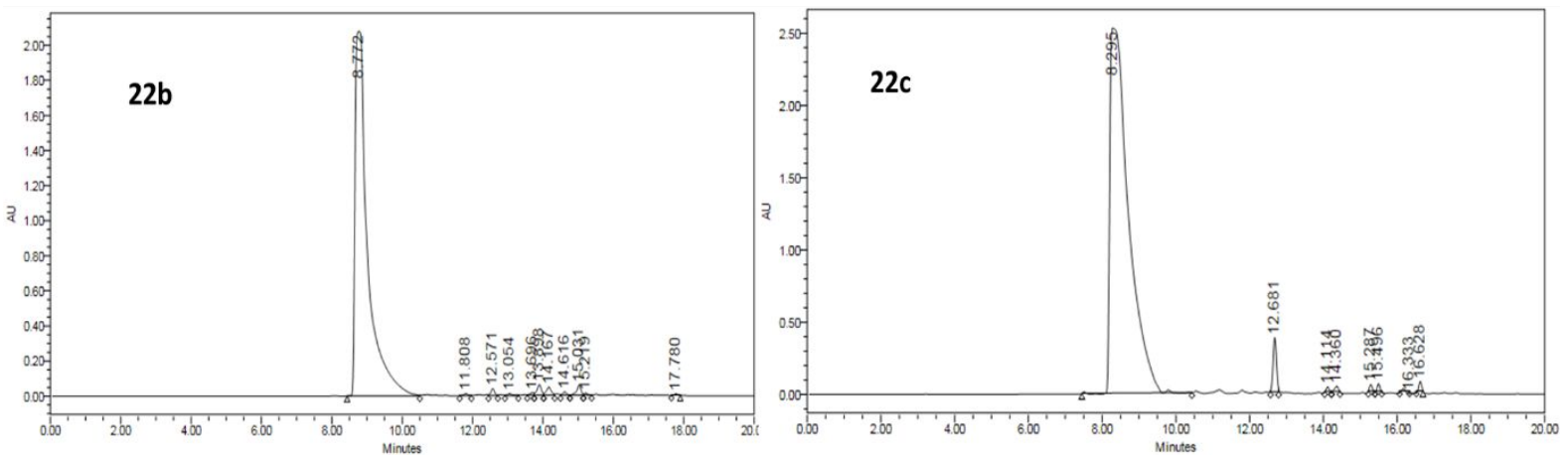

\begin{tabular}{|r|c|r|r|r|}
\hline & \multicolumn{1}{|c|}{ RT } & \multicolumn{1}{|c|}{ Area } & \% Area & Height \\
\hline 1 & 8.772 & 51569674 & 95.65 & 2078949 \\
\hline 2 & 11.808 & 79997 & 0.15 & 10771 \\
\hline 3 & 12.571 & 279161 & 0.52 & 39140 \\
\hline 4 & 13.054 & 92746 & 0.17 & 10415 \\
\hline 5 & 13.696 & 123393 & 0.23 & 15182 \\
\hline 6 & 13.898 & 530430 & 0.98 & 60985 \\
\hline 7 & 14.167 & 471956 & 0.88 & 47083 \\
\hline
\end{tabular}

\begin{tabular}{|r|r|r|r|r|}
\hline & \multicolumn{1}{|c|}{ RT } & \multicolumn{1}{|c|}{ Area } & \% Area & Height \\
\hline 1 & 8.295 & 88903838 & 95.88 & 2532886 \\
\hline 2 & 12.681 & 2385168 & 2.57 & 374793 \\
\hline 3 & 14.114 & 159016 & 0.17 & 30797 \\
\hline 4 & 14.360 & 247279 & 0.27 & 34929 \\
\hline 5 & 15.287 & 244103 & 0.26 & 45329 \\
\hline 6 & 15.496 & 255482 & 0.28 & 50072 \\
\hline 7 & 16.333 & 193083 & 0.21 & -19575 \\
\hline
\end{tabular}



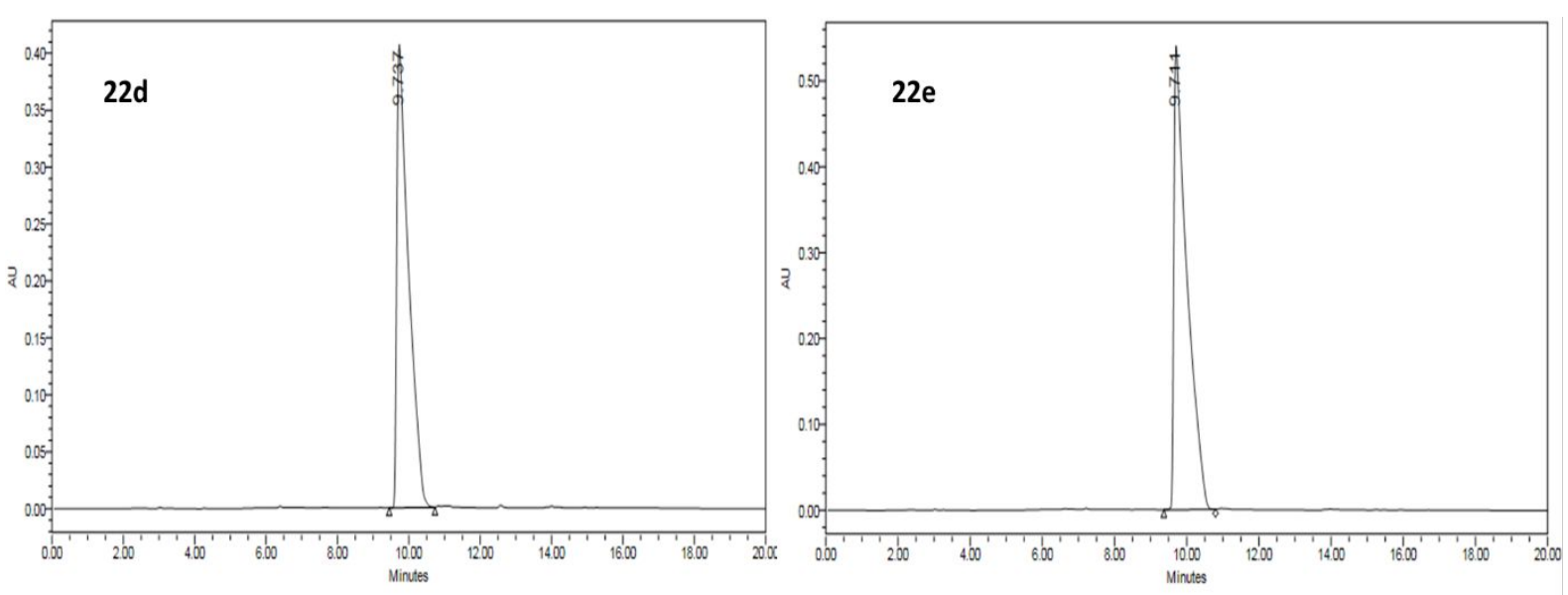

\begin{tabular}{|c|c|c|c|c|}
\hline RT & Area & $\%$ Area & Height \\
\hline 1 & 9.737 & 9240030 & 100.00 & 407422 \\
\hline
\end{tabular}

\begin{tabular}{|c|c|c|c|c|}
\hline & RT & Area & $\%$ Area & Height \\
\hline 1 & 9.711 & 13457934 & 100.00 & 545235 \\
\hline
\end{tabular}
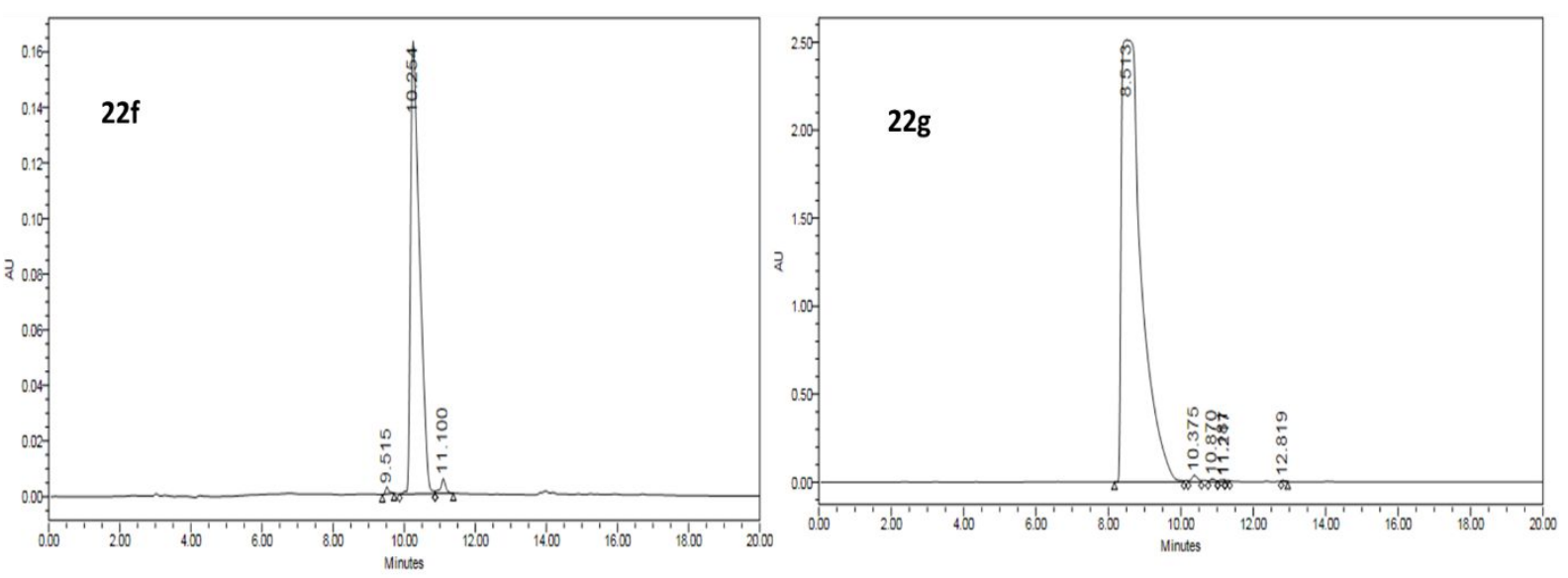

\begin{tabular}{|c|c|r|r|r|}
\hline \multicolumn{1}{|c|}{ RT } & Area & \% Area & Height \\
\hline 1 & 9.515 & 15984 & 0.55 & 2363 \\
\hline 2 & 10.254 & 2834730 & 97.63 & 163028 \\
\hline 3 & 11.100 & 52859 & 1.82 & 5257 \\
\hline
\end{tabular}

\begin{tabular}{|l|l|r|r|r|}
\hline & \multicolumn{1}{|c|}{ RT } & \multicolumn{1}{c|}{ Area } & \% Area & Height \\
\hline 1 & 8.513 & 96933988 & 99.19 & 2510709 \\
\hline 2 & 10.375 & 426788 & 0.44 & 36349 \\
\hline 3 & 10.870 & 157942 & 0.16 & 16428 \\
\hline 4 & 11.181 & 102406 & 0.10 & 10154 \\
\hline 5 & 11.217 & 58472 & 0.06 & 8845 \\
\hline 6 & 12.819 & 47112 & 0.05 & 7805 \\
\hline
\end{tabular}
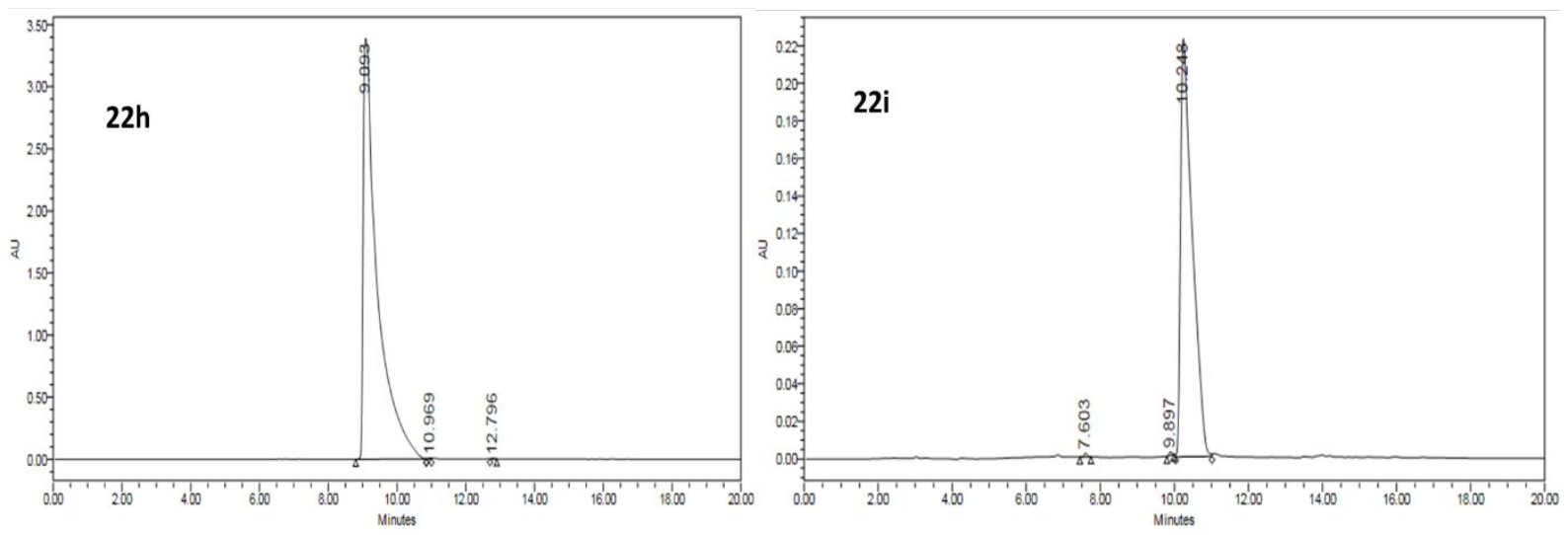

\begin{tabular}{|l|l|r|r|r|}
\hline & \multicolumn{1}{|c|}{ RT } & \multicolumn{1}{c|}{ Area } & \% Area & Height \\
\hline 1 & 9.093 & 96513950 & 99.92 & 3402510 \\
\hline 2 & 10.969 & 50236 & 0.05 & 8500 \\
\hline 3 & 12.796 & 25722 & 0.03 & 3956 \\
\hline
\end{tabular}

\begin{tabular}{|c|c|r|r|r|}
\hline & \multicolumn{1}{|c|}{ RT } & \multicolumn{1}{|c|}{ Area } & $\%$ Area & Height \\
\hline 1 & 7.603 & 12386 & 0.25 & 2044 \\
\hline 2 & 9.897 & 9843 & 0.20 & 1748 \\
\hline 3 & 10.248 & 4882431 & 99.55 & 222702 \\
\hline
\end{tabular}



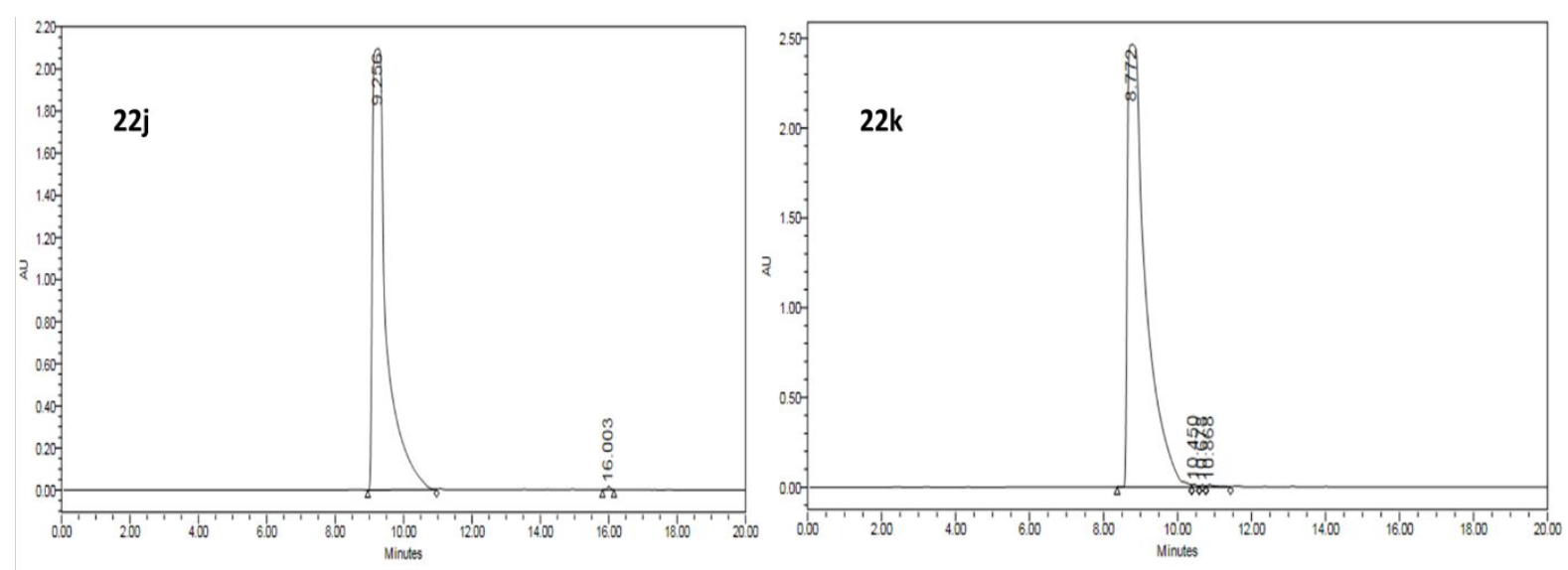

\begin{tabular}{|r|r|r|r|r|}
\hline & \multicolumn{1}{|c|}{ RT } & \multicolumn{1}{c|}{ Area } & \% Area & Height \\
\hline 1 & 8.772 & 84980336 & 99.54 & 2463787 \\
\hline 2 & 10.450 & 126687 & 0.15 & 15517 \\
\hline 3 & 10.678 & 84051 & 0.10 & 11239 \\
\hline 4 & 10.868 & 181073 & 0.21 & 13284 \\
\hline
\end{tabular}

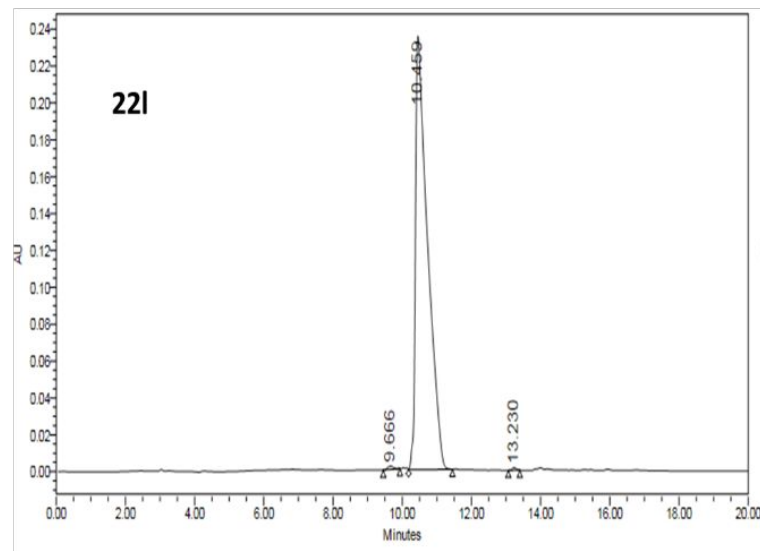

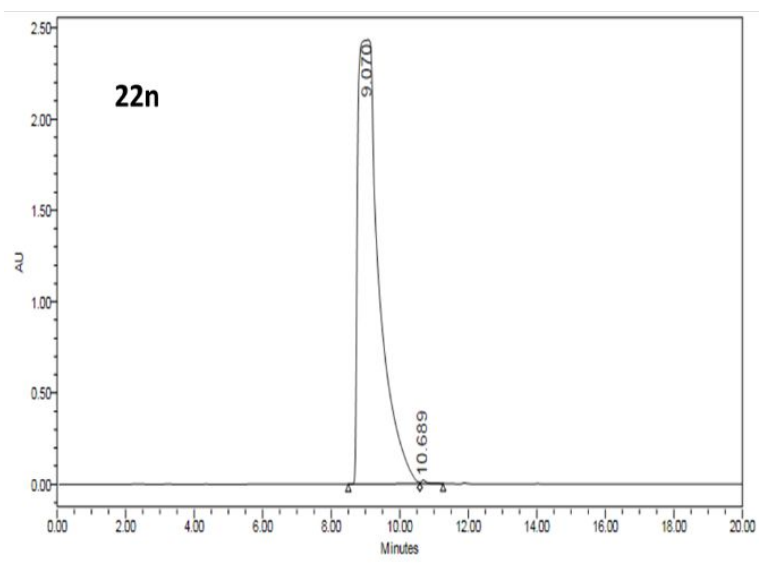

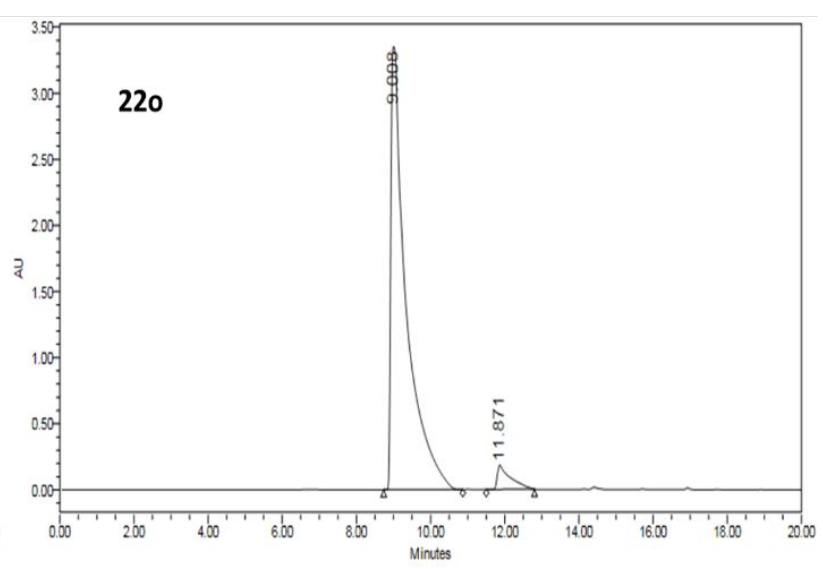

\begin{tabular}{|c|c|r|r|r|}
\cline { 2 - 5 } & \multicolumn{1}{c|}{ RT } & \multicolumn{1}{c|}{ Area } & \% Area & Height \\
\hline 1 & 9.070 & 105411098 & 99.79 & 2432234 \\
\hline 2 & 10.689 & 226422 & 0.21 & 20411 \\
\hline
\end{tabular}

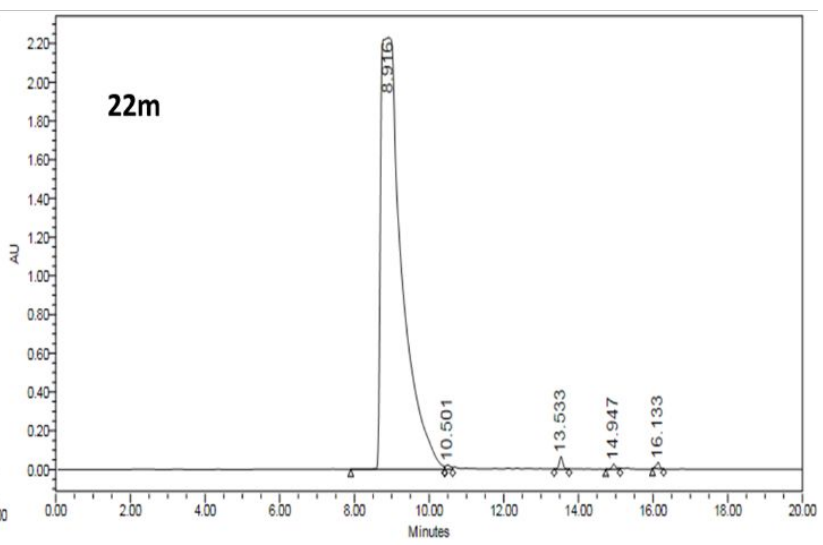

\begin{tabular}{|l|r|r|r|r|}
\hline & \multicolumn{1}{|c|}{ RT } & \multicolumn{1}{c|}{ Area } & $\%$ Area & Height \\
\hline 1 & 8.916 & 88035642 & 98.81 & 2228734 \\
\hline 2 & 10.501 & 210470 & 0.24 & 21754 \\
\hline 3 & 13.533 & 434821 & 0.49 & 63887 \\
\hline 4 & 14.947 & 191432 & 0.21 & 25267 \\
\hline 5 & 16.133 & 226910 & 0.25 & 31945 \\
\hline
\end{tabular}

\begin{tabular}{|c|c|c|r|r|}
\hline & RT & Area & \% Area & Height \\
\hline 1 & 9.008 & 95787588 & 95.53 & 3363487 \\
\hline 2 & 11.871 & 4487220 & 4.47 & 182171 \\
\hline
\end{tabular}



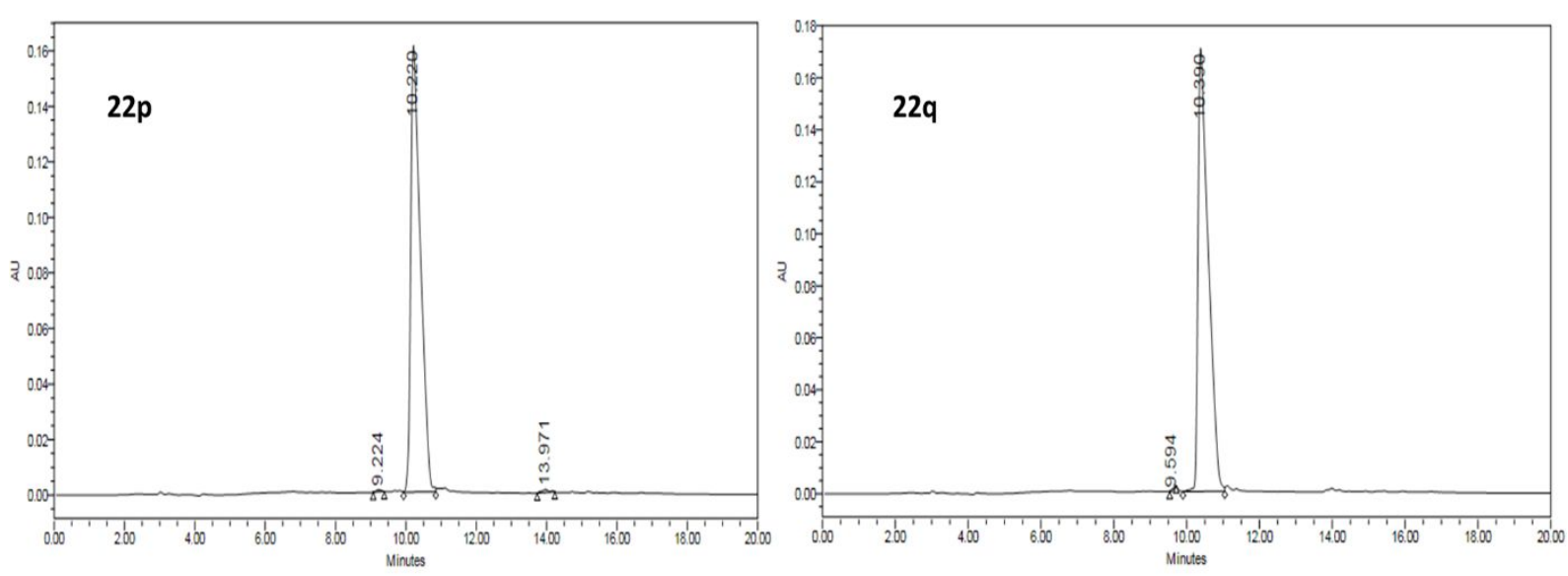

\begin{tabular}{|l|c|r|r|r|}
\hline & RT & Area & \% Area & Height \\
\hline 1 & 9.224 & 7709 & 0.25 & 756 \\
\hline 2 & 10.220 & 3068840 & 99.25 & 160930 \\
\hline 3 & 13.971 & 15471 & 0.50 & 1107 \\
\hline
\end{tabular}

\begin{tabular}{|l|c|r|r|r|}
\hline & \multicolumn{1}{|c|}{ RT } & Area & $\%$ Area & Height \\
\hline 1 & 9.594 & 4850 & 0.15 & -731 \\
\hline 2 & 10.390 & 3325080 & 99.85 & 171105 \\
\hline
\end{tabular}
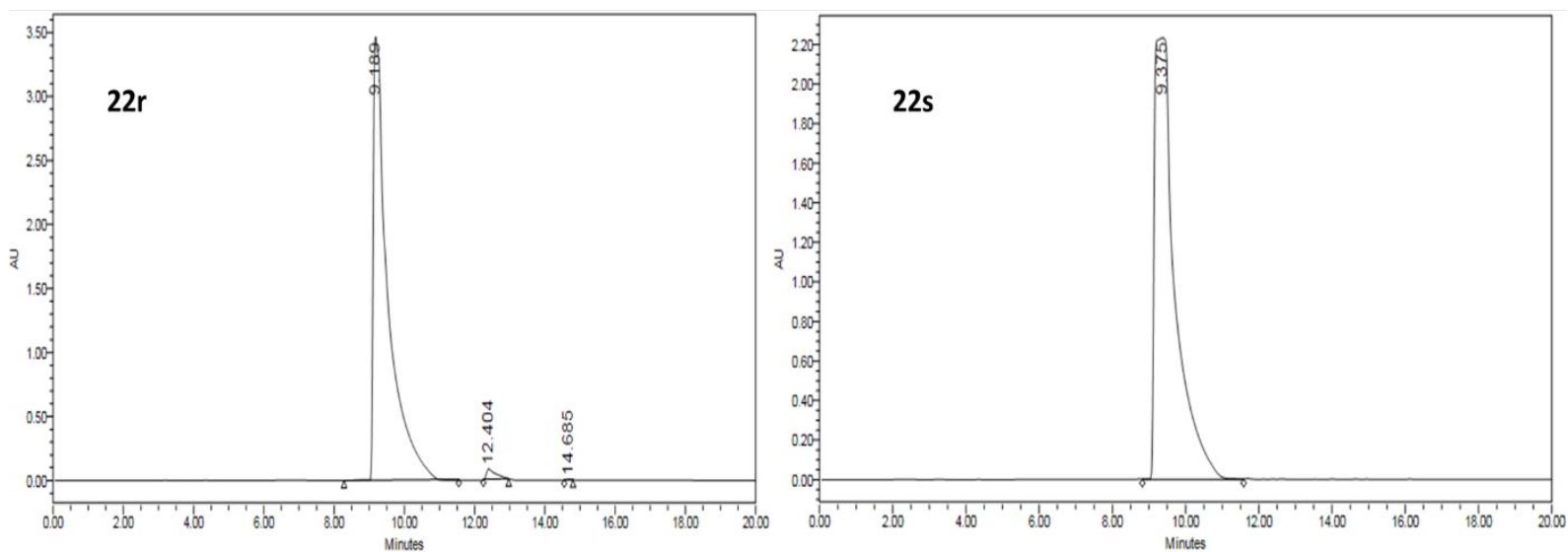

\begin{tabular}{|r|c|r|r|r|}
\hline & RT & \multicolumn{1}{|c|}{ Area } & \% Area & Height \\
\hline 1 & 9.189 & 100933390 & 98.56 & 3470170 \\
\hline 2 & 12.404 & 1426432 & 1.39 & 78982 \\
\hline 3 & 14.685 & 43363 & 0.04 & 5554 \\
\hline
\end{tabular}

\begin{tabular}{|l|l|l|l|l|}
\hline RT & Area & $\%$ Area & Height \\
\hline
\end{tabular}

\begin{tabular}{l|l|l|l|l|l|}
\hline 1 & 9.375 & 87994724 & 100.00 & 2232115 \\
\hline
\end{tabular}
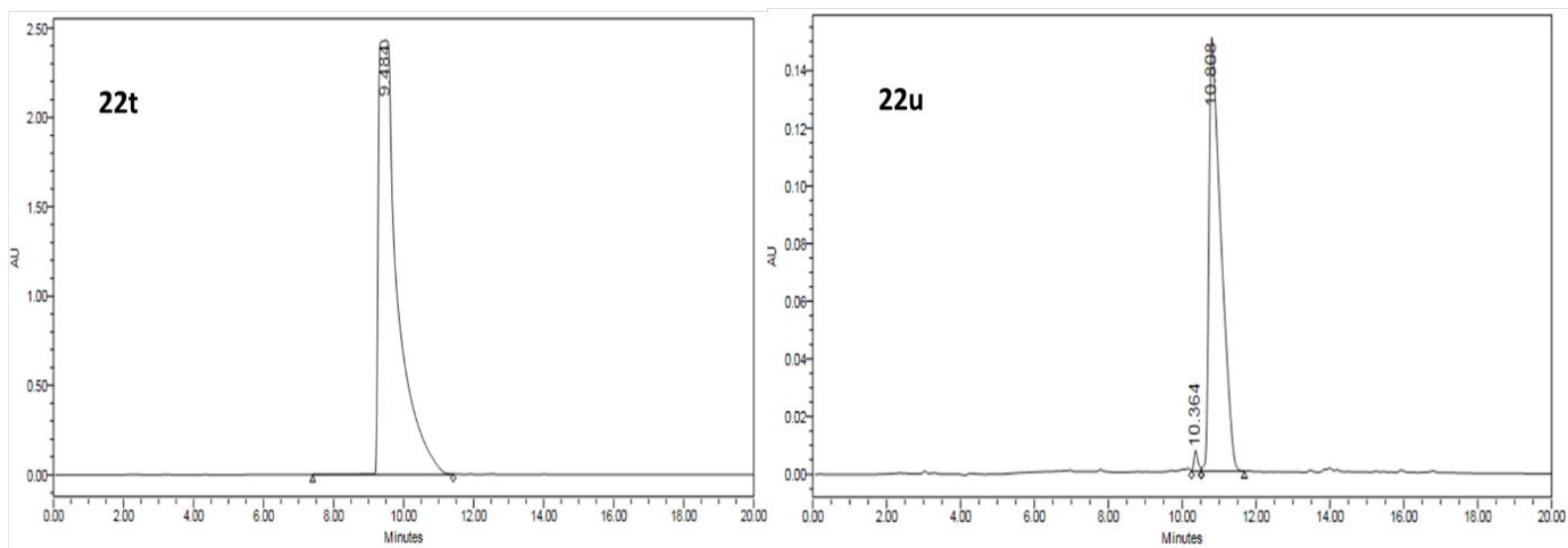

\begin{tabular}{|c|c|c|c|c|}
\hline & RT & Area & $\%$ Area & Height \\
\hline 1 & 9.484 & 92912802 & 100.00 & 2429255 \\
\hline
\end{tabular}

\begin{tabular}{|c|c|r|r|r|}
\cline { 2 - 5 } \multicolumn{1}{|c|}{ RT } & Area & \% Area & Height \\
\hline 1 & 10.364 & 53885 & 1.56 & 7065 \\
\hline 2 & 10.808 & 3393143 & 98.44 & 150930 \\
\hline
\end{tabular} 

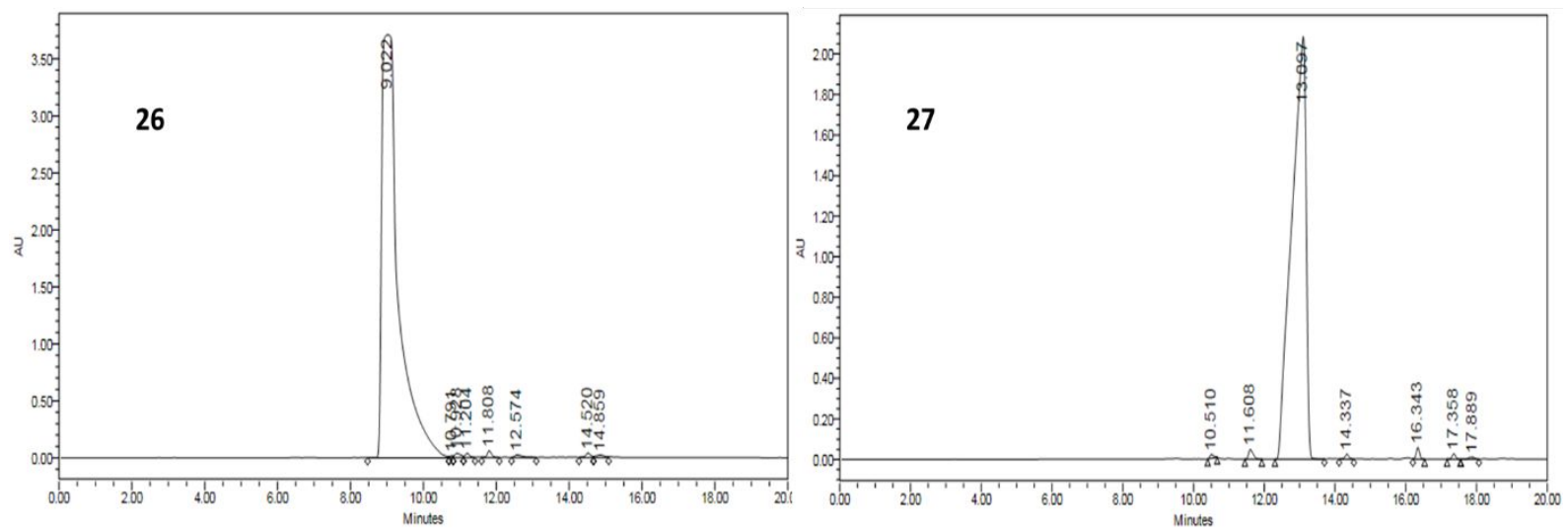

\begin{tabular}{|r|c|r|r|r|}
\cline { 2 - 5 } & \multicolumn{1}{|c|}{ RT } & \multicolumn{1}{c|}{ Area } & \% Area & Height \\
\hline 1 & 9.022 & 119466111 & 97.90 & 3710840 \\
\hline 2 & 10.791 & 95792 & 0.08 & 15555 \\
\hline 3 & 10.928 & 454868 & 0.37 & 38583 \\
\hline 4 & 11.204 & 338258 & 0.28 & 39076 \\
\hline 5 & 11.808 & 530381 & 0.43 & 59296 \\
\hline 6 & 12.574 & 380893 & 0.31 & 24544 \\
\hline 7 & 14.520 & 401361 & 0.33 & 40132 \\
\hline
\end{tabular}

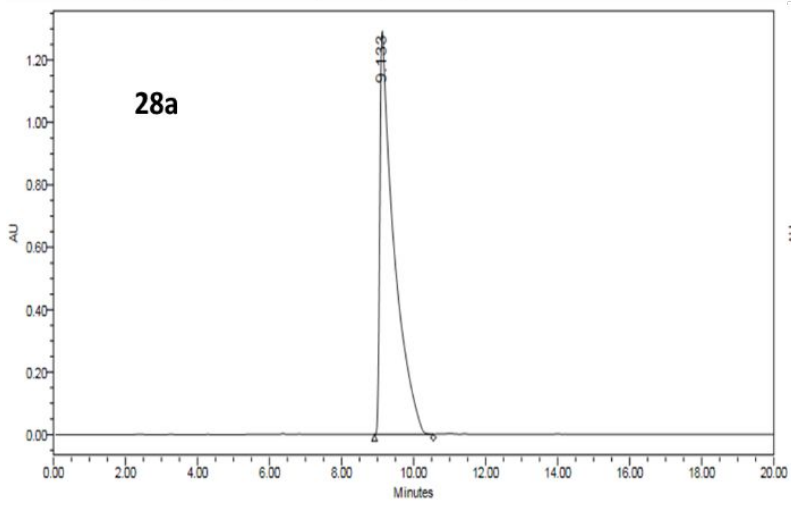

\begin{tabular}{|r|c|r|r|r|}
\hline & RT & Area & $\%$ Area & Height \\
\hline 1 & 10.510 & 138243 & 0.23 & 18279 \\
\hline 2 & 11.608 & 425404 & 0.71 & 48678 \\
\hline 3 & 13.097 & 58522039 & 97.58 & 2085873 \\
\hline 4 & 14.337 & 199131 & 0.33 & 23997 \\
\hline 5 & 16.343 & 381941 & 0.64 & 56735 \\
\hline 6 & 17.358 & 185859 & 0.31 & 25399 \\
\hline 7 & 17.889 & 120226 & 0.20 & 9396 \\
\hline
\end{tabular}

\begin{tabular}{|c|c|c|c|}
\hline RT & Area & $\%$ Area & Height \\
\hline
\end{tabular}

\begin{tabular}{|l|l|l|l|l|}
\hline 1 & 9.133 & 34961672 & 100.00 & 1291948 \\
\hline
\end{tabular}
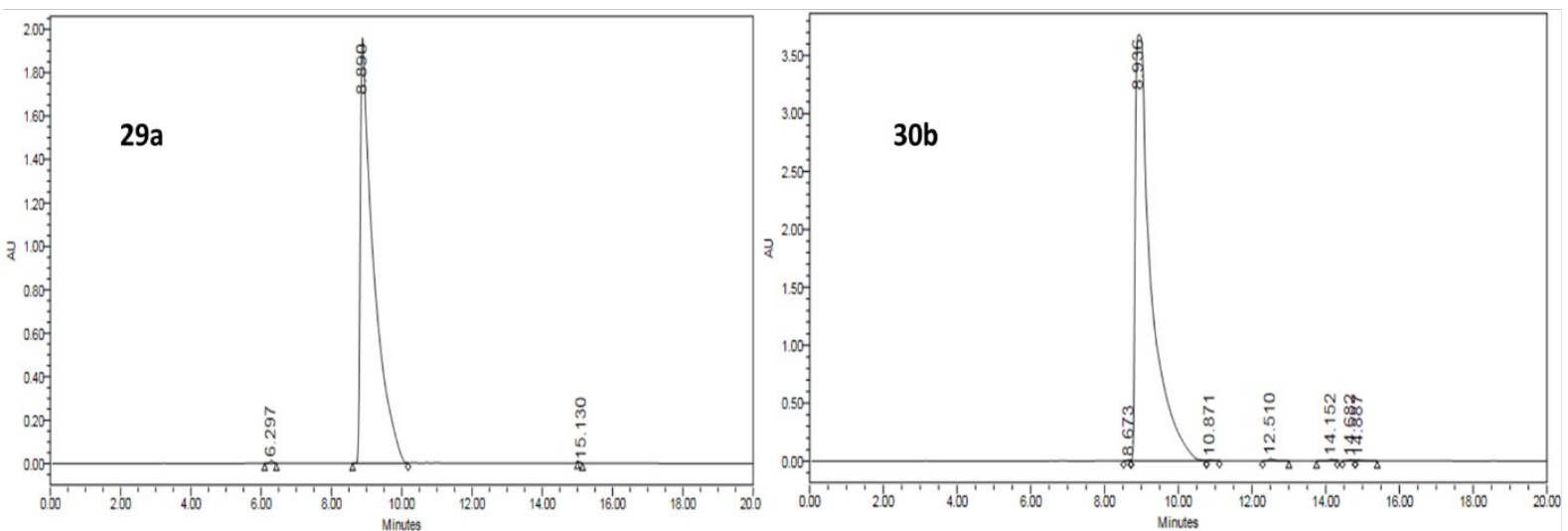

\begin{tabular}{|l|c|r|r|r|}
\hline & RT & \multicolumn{1}{|c|}{ Area } & $\%$ Area & Height \\
\hline 1 & 6.297 & 74556 & 0.14 & 13185 \\
\hline 2 & 8.890 & 51731165 & 99.84 & 1965293 \\
\hline 3 & 15.130 & 9810 & 0.02 & -1058 \\
\hline
\end{tabular}

\begin{tabular}{|r|c|r|r|r|}
\hline & RT & \multicolumn{1}{|c|}{ Area } & \% Area & Height \\
\hline 1 & 8.673 & 53983 & 0.05 & 7817 \\
\hline 2 & 8.936 & 111063615 & 99.36 & 3676459 \\
\hline 3 & 10.871 & 74952 & 0.07 & 7595 \\
\hline 4 & 12.510 & 187142 & 0.17 & 16318 \\
\hline 5 & 14.152 & 184006 & 0.16 & 14511 \\
\hline 6 & 14.682 & 120157 & 0.11 & 9919 \\
\hline 7 & 14.887 & 96539 & 0.09 & 6249 \\
\hline
\end{tabular}



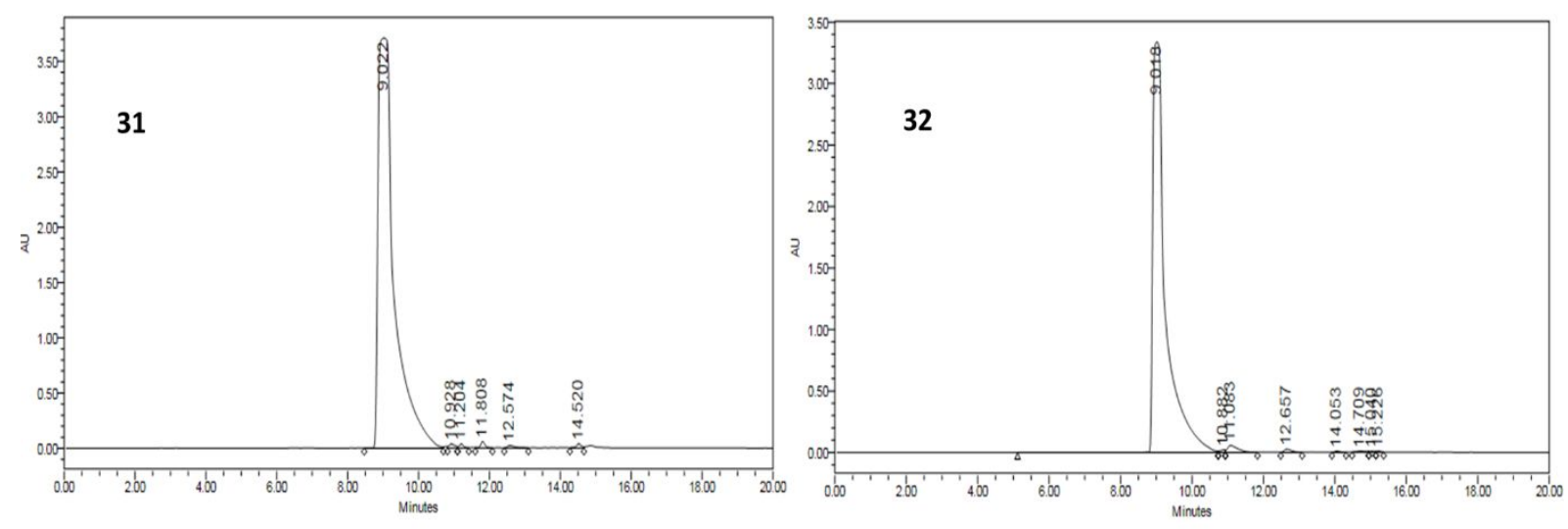

\begin{tabular}{|r|r|r|r|r|}
\hline & \multicolumn{1}{|c|}{ RT } & \multicolumn{1}{c|}{ Area } & \% Area & Height \\
\hline 1 & 9.022 & 119466111 & 98.27 & 3710840 \\
\hline 2 & 10.928 & 454868 & 0.37 & 38583 \\
\hline 3 & 11.204 & 338258 & 0.28 & 39076 \\
\hline 4 & 11.808 & 530381 & 0.44 & 59296 \\
\hline 5 & 12.574 & 380893 & 0.31 & 24544 \\
\hline 6 & 14.520 & 401361 & 0.33 & 40132 \\
\hline
\end{tabular}

\begin{tabular}{|r|r|r|r|r|}
\hline & \multicolumn{1}{|c|}{ RT } & \multicolumn{1}{c|}{ Area } & \% Area & Height \\
\hline 1 & 9.018 & 83244080 & 97.39 & 3336851 \\
\hline 2 & 10.882 & 193585 & 0.23 & 20456 \\
\hline 3 & 11.083 & 1167883 & 1.37 & 57126 \\
\hline 4 & 12.657 & 383319 & 0.45 & 26323 \\
\hline 5 & 14.053 & 110253 & 0.13 & 10140 \\
\hline 6 & 14.709 & 205086 & 0.24 & 10776 \\
\hline 7 & 15.040 & 89908 & 0.11 & 8230 \\
\hline
\end{tabular}
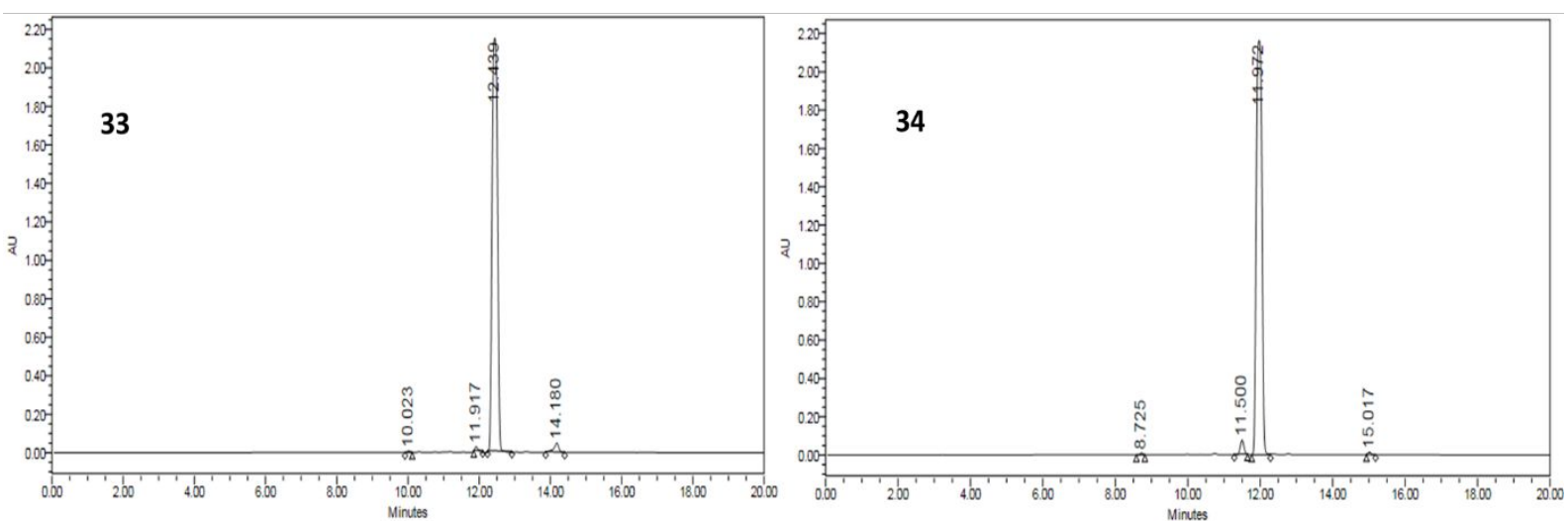

\begin{tabular}{|r|c|r|r|r|}
\hline & RT & Area & $\%$ Area & Height \\
\hline 1 & 10.023 & 42704 & 0.18 & 7595 \\
\hline 2 & 11.917 & 142487 & 0.61 & 18306 \\
\hline 3 & 12.439 & 22631693 & 97.36 & 2151058 \\
\hline 4 & 14.180 & 428446 & 1.84 & 46288 \\
\hline
\end{tabular}

\begin{tabular}{|r|c|r|r|r|}
\hline & RT & \multicolumn{1}{|c|}{ Area } & \% Area & Height \\
\hline 1 & 8.725 & 50788 & 0.21 & 8974 \\
\hline 2 & 11.500 & 515188 & 2.16 & 74251 \\
\hline 3 & 11.972 & 23253384 & 97.32 & 2166027 \\
\hline 4 & 15.017 & 75501 & 0.32 & 12647 \\
\hline
\end{tabular}

